\title{
Fondamental Physics at Low Energies
}

\author{
Joerg Jaeckel
}

Institute for Particle Physics Phenomenology

Durham - United Kingdom 


\section{Fundamental Physics}

@

Low Energies

Joerg Jaeckel

IPPP Durham

For a review see, JJ and A. Ringwald 1002.0329 
Where we want to go...

$m(1 P 3) m$

The Standard Model

\section{The Hidden Sector}

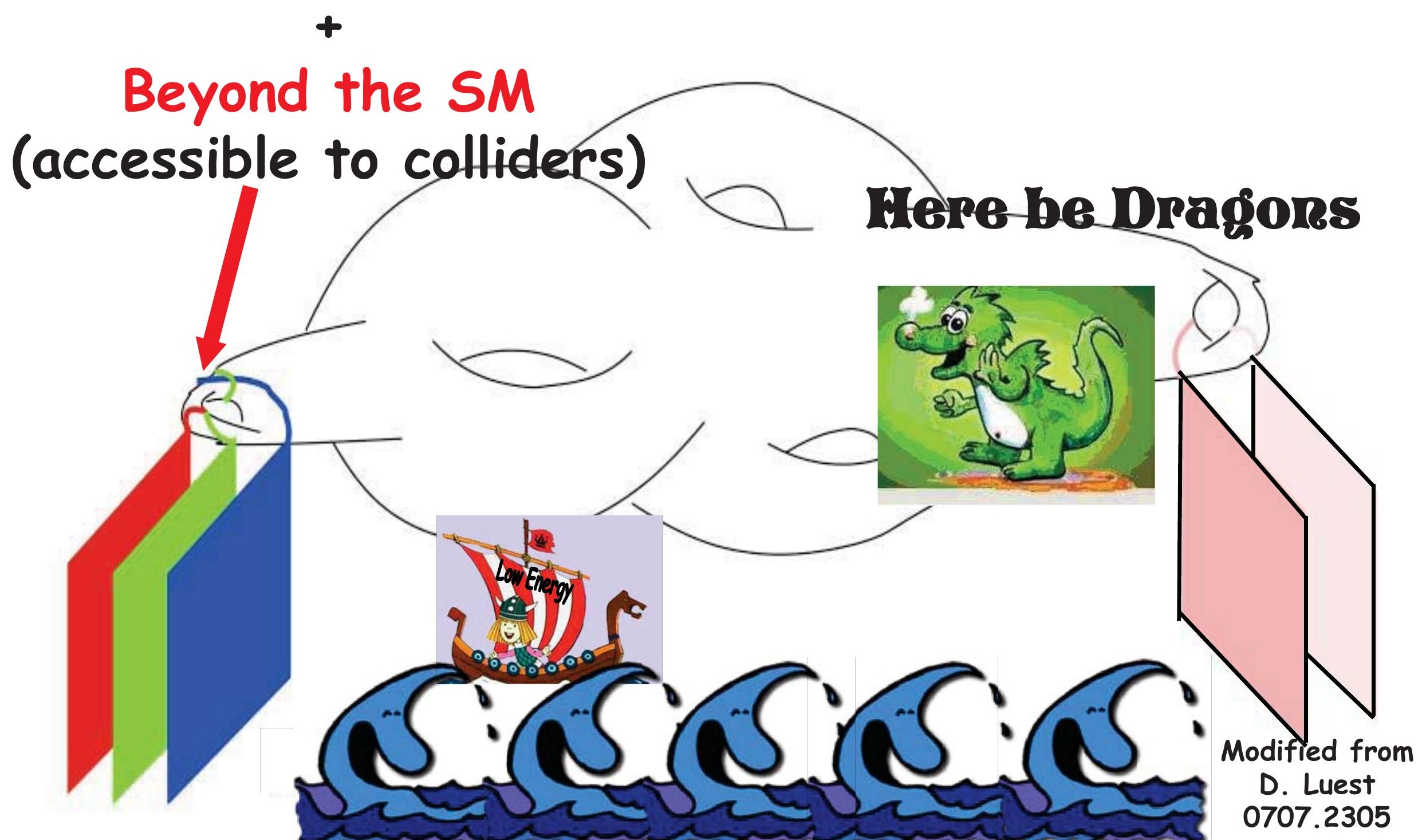




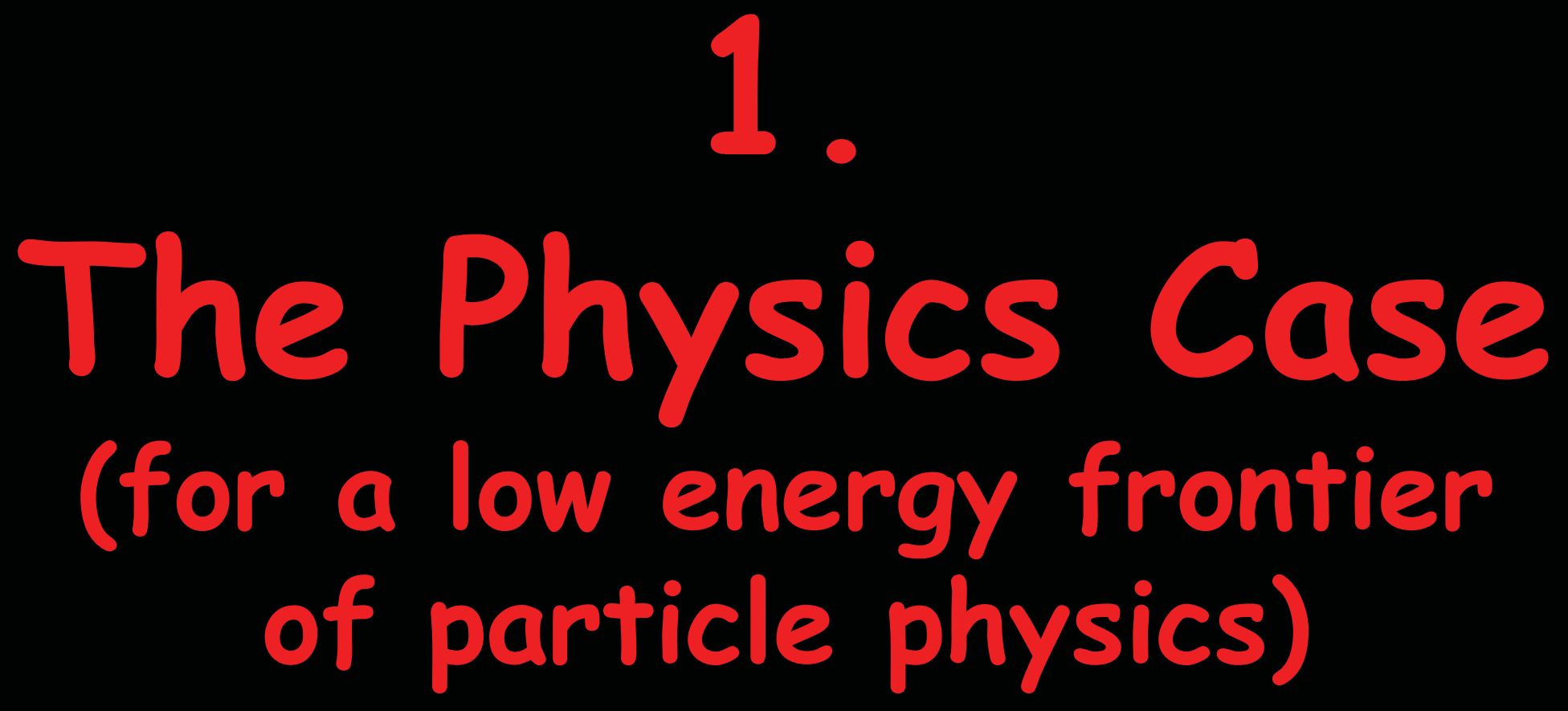




\section{The Standard Model}

$m(1 / P 3) m$

University of Durham

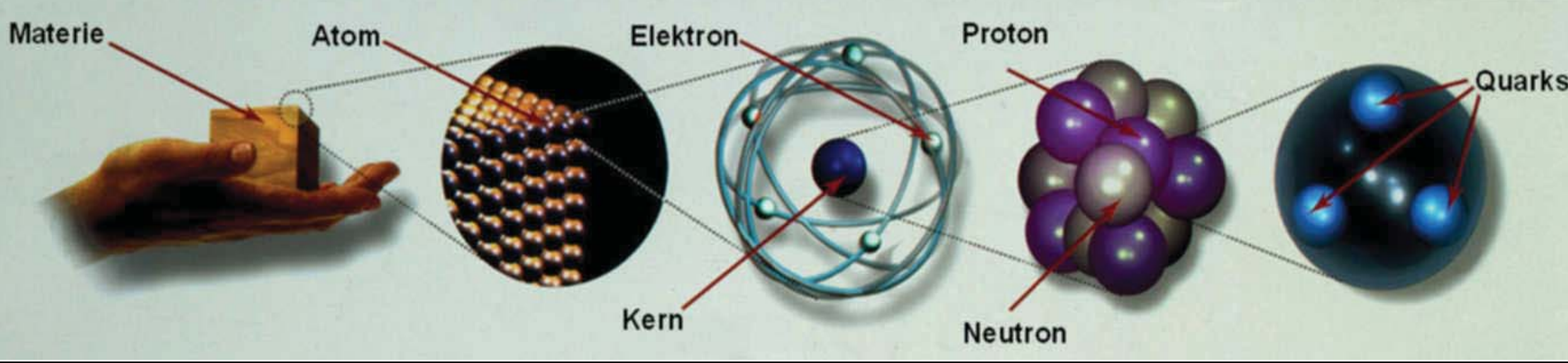

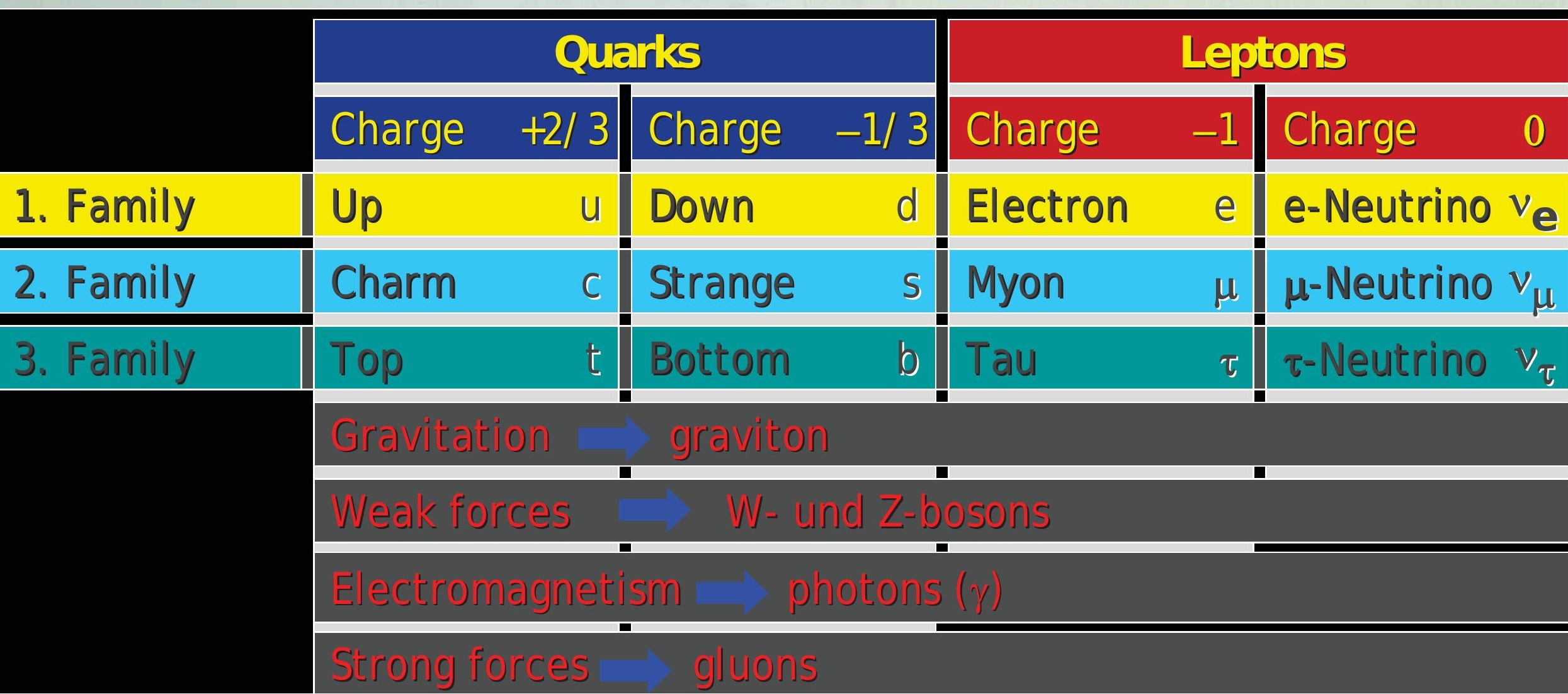




$$
\begin{aligned}
& \text { We need... } \\
& \text { Physics beyond the } \\
& \text { Standard Model }
\end{aligned}
$$


Hints for new Physics 


\section{Uglyness of old models}

- The Standard Model has many free parameters: $O(30)$

- Naturalness problems. Finetuning.

Examples:

Higgs mass, $\theta$-angle (strong CP-problem) 
A dirty little secret...

$S=\int d^{4} x\left[-\frac{1}{4} G^{\mu \nu} G_{\mu \nu}-\frac{\theta}{4} G^{\mu \nu} \tilde{G}_{\mu \nu}\right.$

$$
\left.+\mathrm{l} \bar{\psi} D_{\mu} \gamma^{\mu} \psi+\bar{\psi} M \psi\right]
$$

- The $\theta$-term is CP violating!

- Connected to strong interactions!

\section{Measure electric dipole moment of the neutron!}




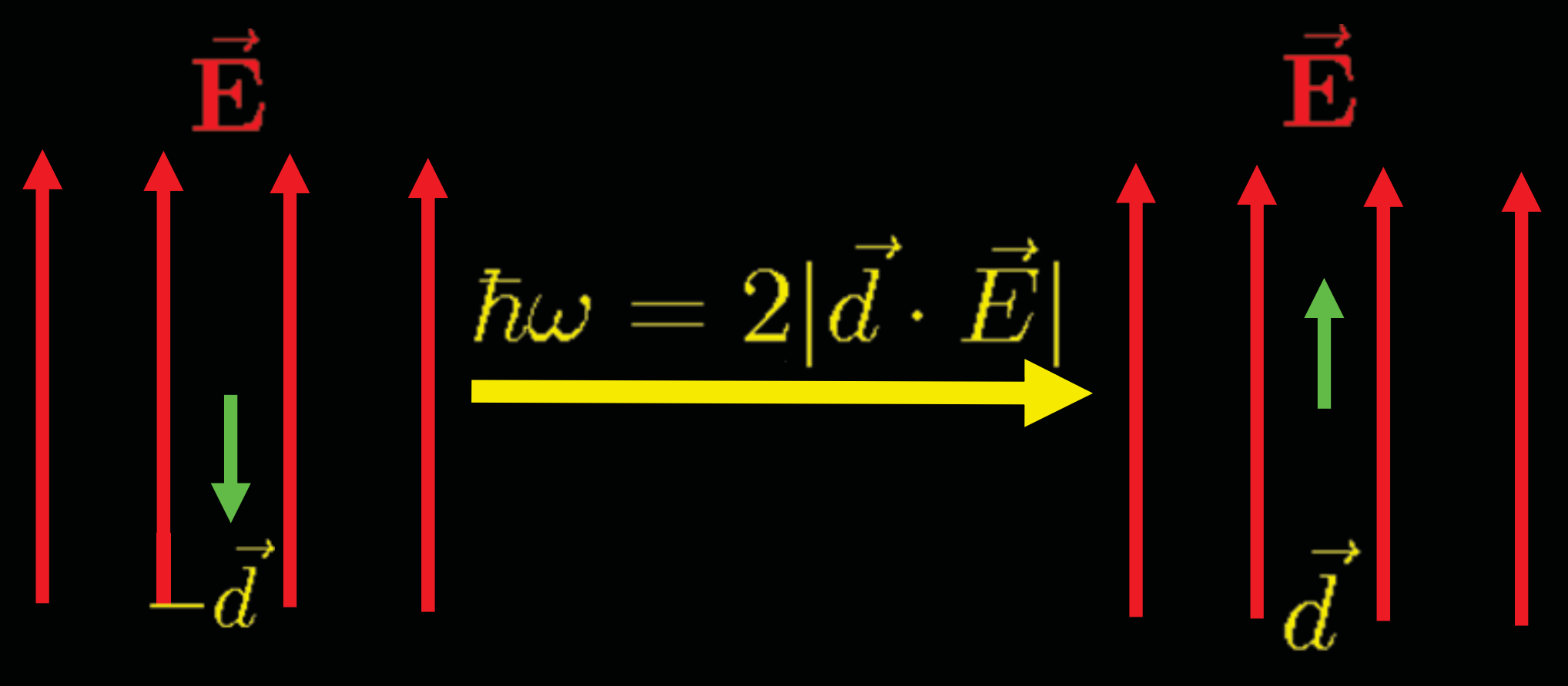

Measure transition frequency. 


\section{No neutron electric dipole moment...}
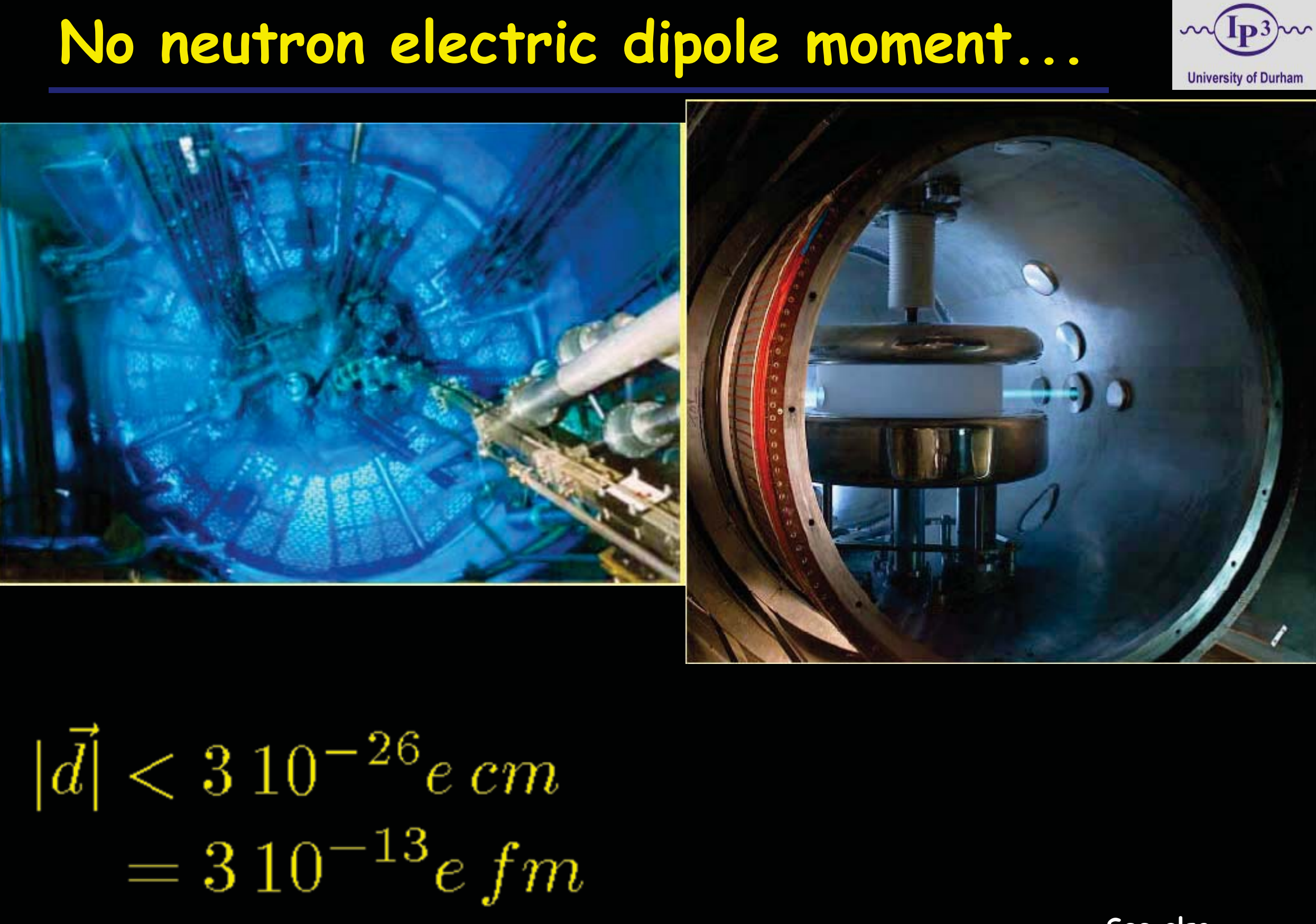

See also https://neutronedm.org 


\section{No neutron electric dipole moment...}

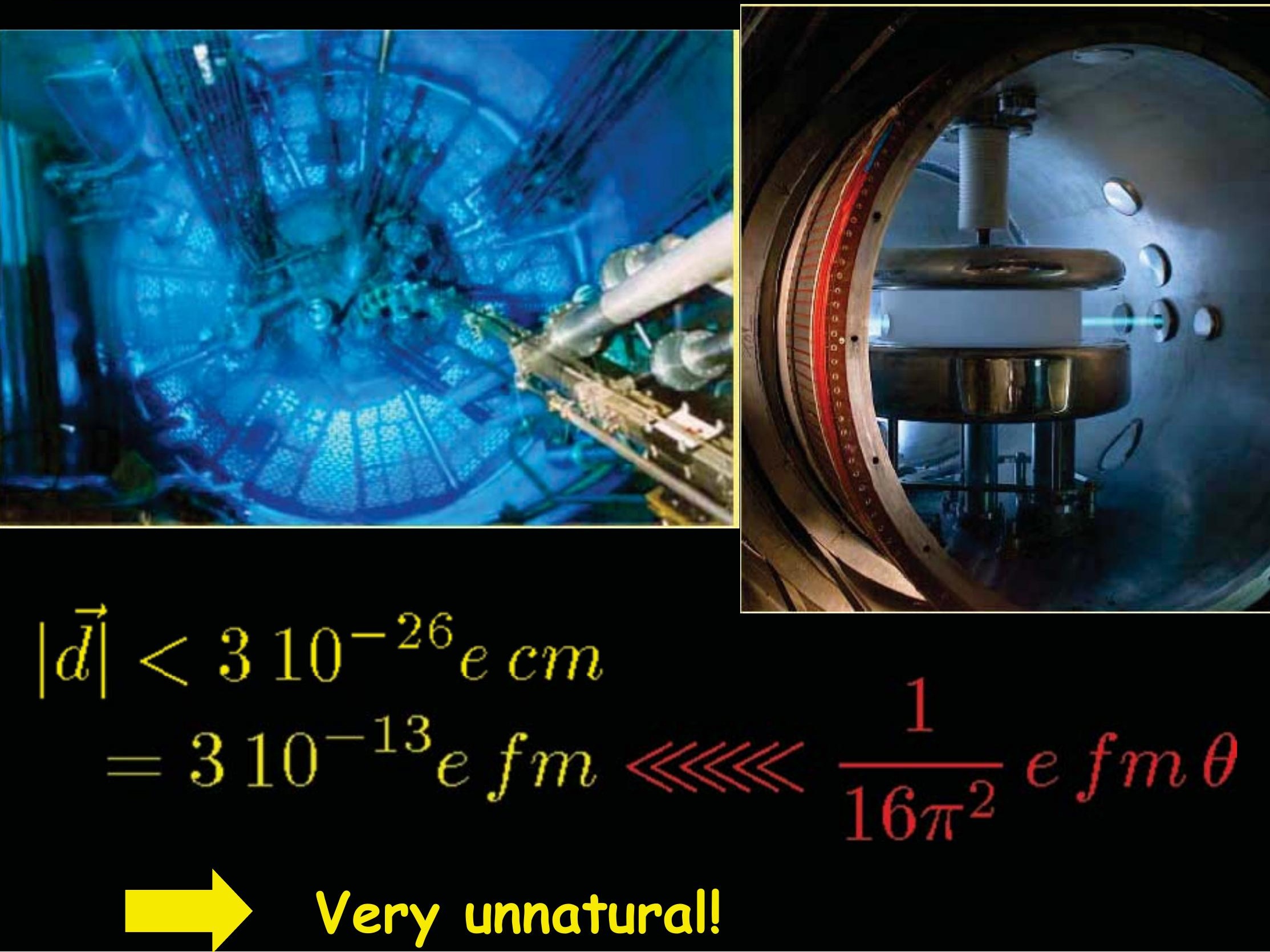




\section{More precisely}

- Detailed calculation gives

see e.g., J.E. Kim Phys.Rept. 1501987

$|\vec{d}| \sim 1-10 \times 10^{-16} \operatorname{ecm} \theta$

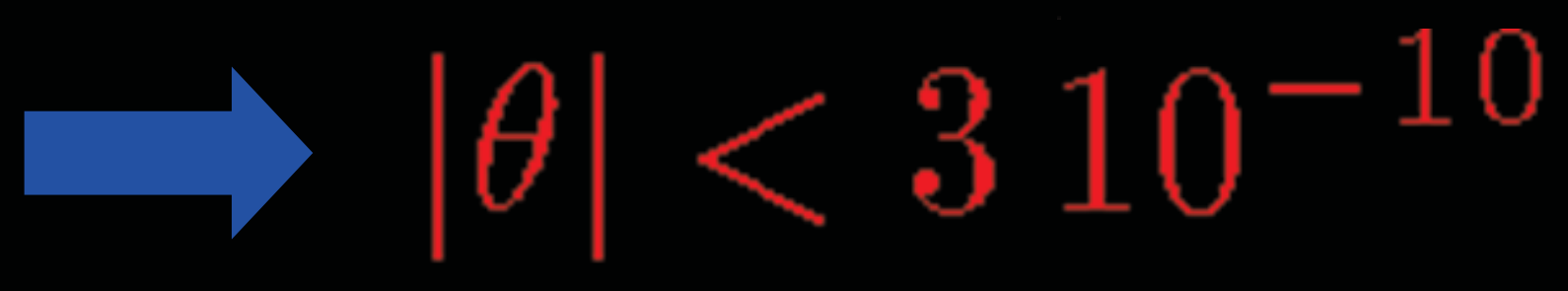

Extremely unnatural! 


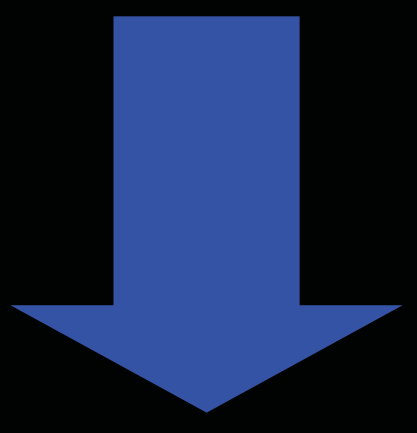

Strong CP Problem 


\section{Uglyness of old models}

- The Standard Model has many free parameters: $O(30)$

- Naturalness problems. Finetuning.

Examples:

Higgs mass, $\theta$-angle (strong CP-problem)

- Gravity separate, i.e. not unified.

- (Probably) Breaks down at a finite energy scale

Landau poles etc. 


\section{Unexplained Stuff}

- Dark Matter (25\%)

(astrophysical + cosmological observations) 


\section{Gravitational lenses}

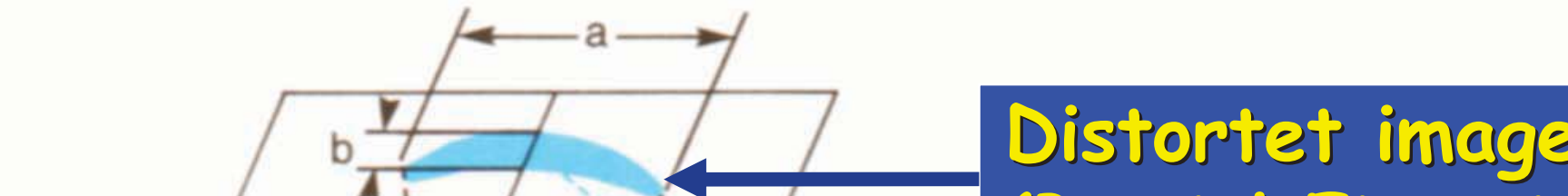

(Partial Einstein Ring)

Far away galxy

see

http://ned.ipac.caltech.edu/ level5/Tyson2/Tyson2.html
Galaxy cluster

(„Gravitational lense $)$ Observer 
Gravitationslinseń Abell 2218

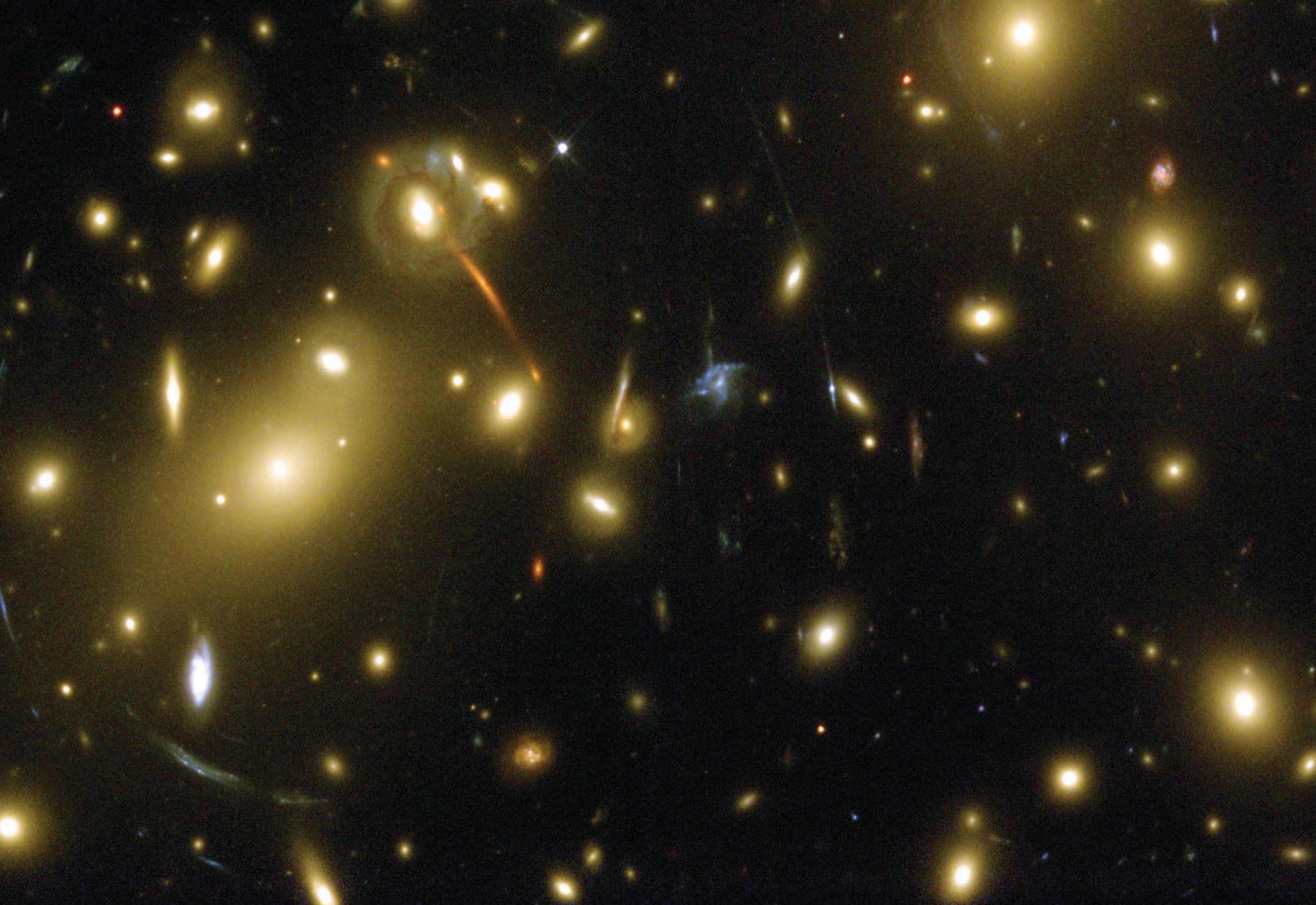




\section{Unexplained Stuff}

- Dark Matter (25\%)

(astrophysical + cosmological observations)

- Dark Energy (70\%) (astrophysical + cosmological observations)

- Mass Hierarchies

(colliders, neutrino exp, etc)

- Small parameters ( $\theta$-angle, again) (neutron electric dipole measurements) 


\section{BSM physics @ Work?}

- $(g-2)_{\mu}$ deviations from $S M$ prediction

- DAMA anomaly

- CogeNT etc.

- PAMELA + Fermi observation

- WMAP observes extra "neutrinos"

- Proton radius in muonic hydrogen 
Hints for new Physics

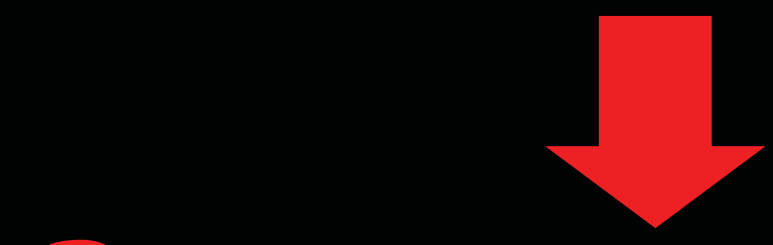

Bottom-up

(pheno)
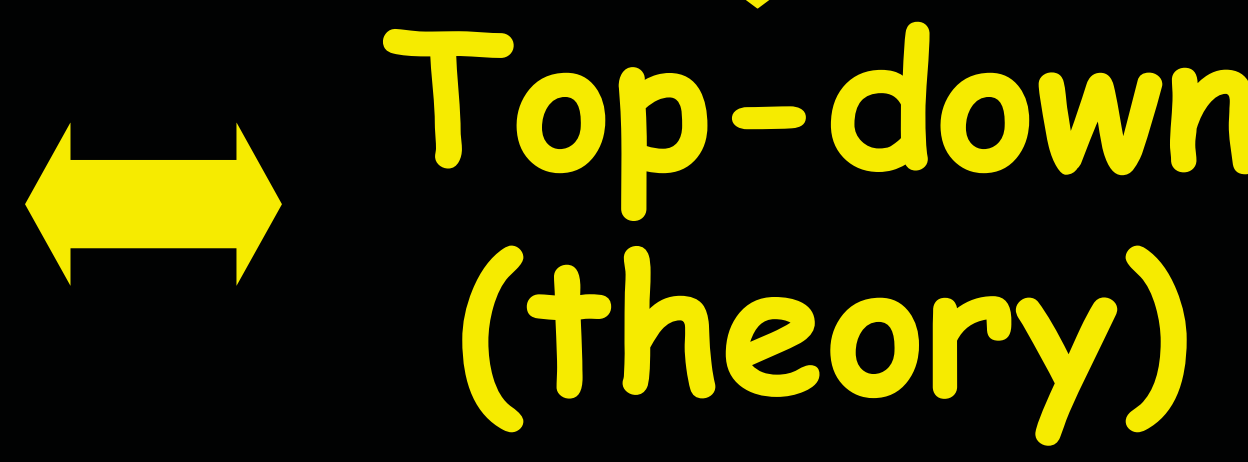

Fix problem

'here and now'

Go back to drawing board 'Start from scratch' 


$$
\text { The Axion }
$$




\section{Commercials}


Your CP is violated too strongly? 


\section{Use Axion!}

$m \mathbb{P}^{3} \sim$

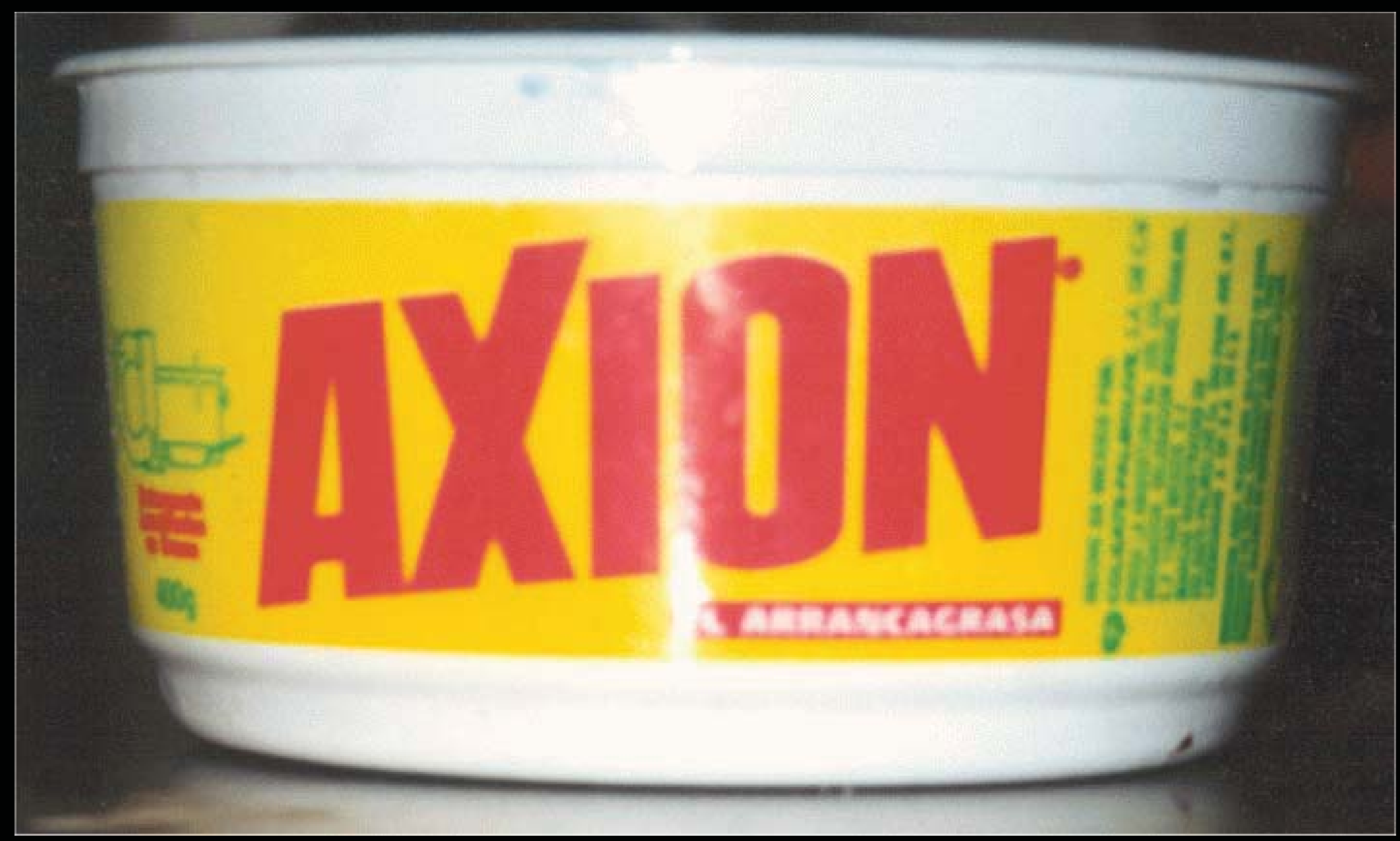

New! With special cleaning particles and extra strong photon coupling. 
As we have seen...

$m\left(1 P^{2} n\right.$

$S=\int d^{4} x\left[-\frac{1}{4} G^{\mu \nu} G_{\mu \nu}-\frac{\theta}{4} G^{\mu \nu} \tilde{G}_{\mu \nu}\right.$

$$
\left.+\mathrm{l} \bar{\psi} D_{\mu} \gamma^{\mu} \psi+\bar{\psi} M \psi\right]
$$

No electric dipole moment of the neutron! $\Rightarrow|\theta|<310^{-10}$

Need an explanation! 


\section{A Dynamical $\theta$}

- Idea: see e.g., J.E. Kim Phys.Rept. 1501987 and references therein

- Make $\theta$ a dynamical degree of freedom $a$.

- Let a have no tree level potential

- Let a have only derivative couplings

- Then:

$\Rightarrow V[0] \leq V[a] \quad \forall a$

a will evolve to $a=\theta=0$

$C P$ is conserved 
- Properties:

- Let a be a dynamical degree of freedom.

- Let a have no tree level potential

- Let a have only derivative couplings

- $\mathbf{a} / \mathbf{f}_{\mathrm{a}} \in[\mathbf{0}, \mathbf{2 \pi}]$ since $\int d^{4} x \frac{G_{\mu \nu} \tilde{G}^{\mu \nu}}{32 \pi^{2}}=n \in \mathbb{Z}$

$a$ is Goldstone boson of a U(1) symmetry

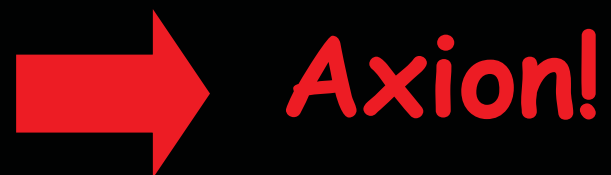

Peccei-Quinn Symmetry 
- $U(1)_{P Q}$ is not exact

\section{Goldstone $\rightarrow$ Pseudogoldstone}

- Dimensional considerations

- SSB scale

- Coupling to $G \tilde{G}$ :

- Scale of explicit breaking $\sim f_{X}$

$\sim \frac{a}{f_{X}} G^{\mu \nu} \tilde{G}_{\mu \nu}$

$\sim \frac{1}{f_{X}} m_{\pi}^{2} f_{\pi}^{2}$

$\Rightarrow$ Goldstone mass $m_{a}^{2} \sim \frac{\Lambda^{4}}{f_{X}^{2}}$ 


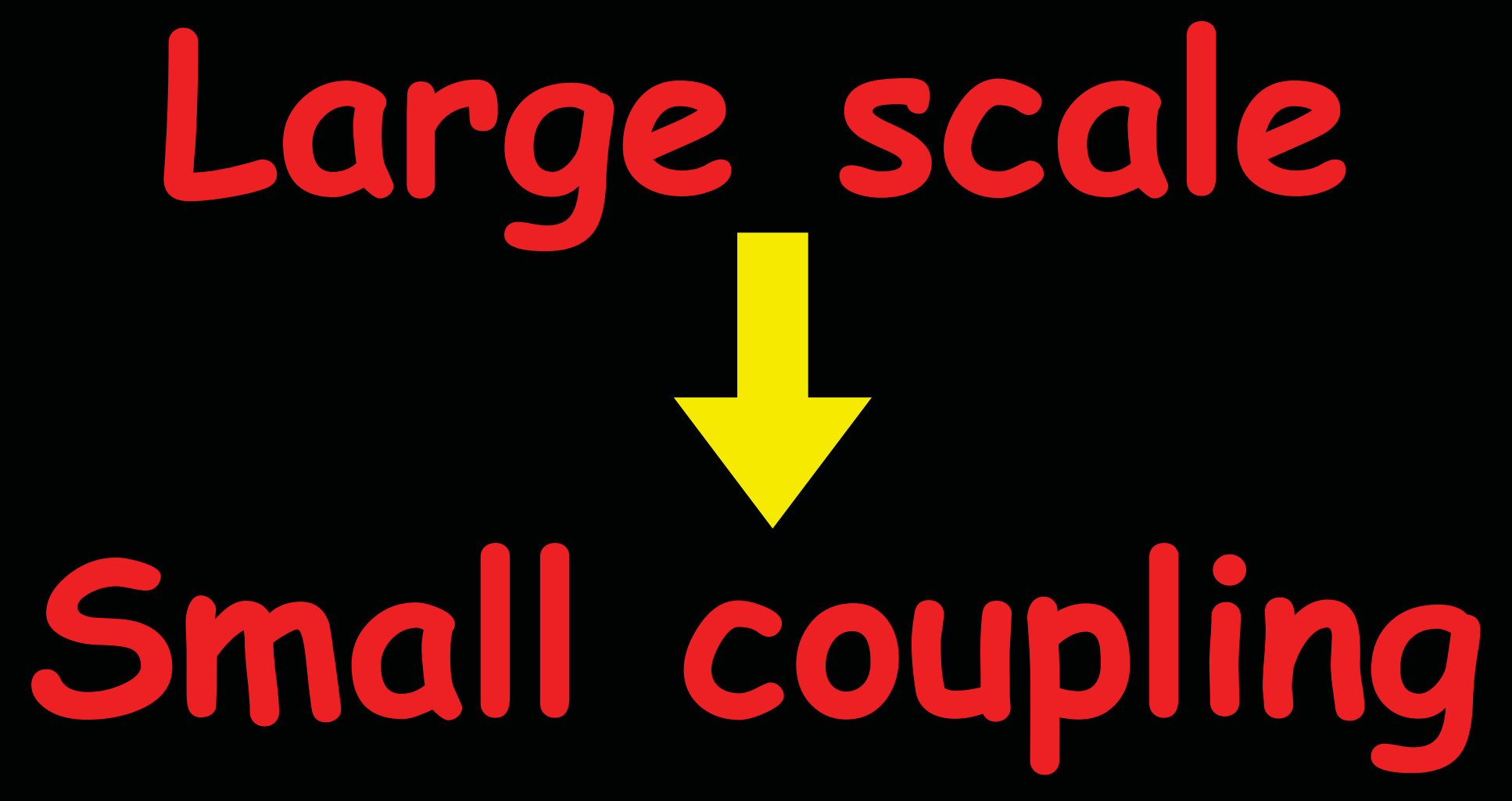


$\mathcal{L}_{\text {Int }}=-\frac{1}{4} g a F^{\mu \nu} \tilde{F}_{\mu \nu}=-g a \mathbf{E} \cdot \mathbf{B}$

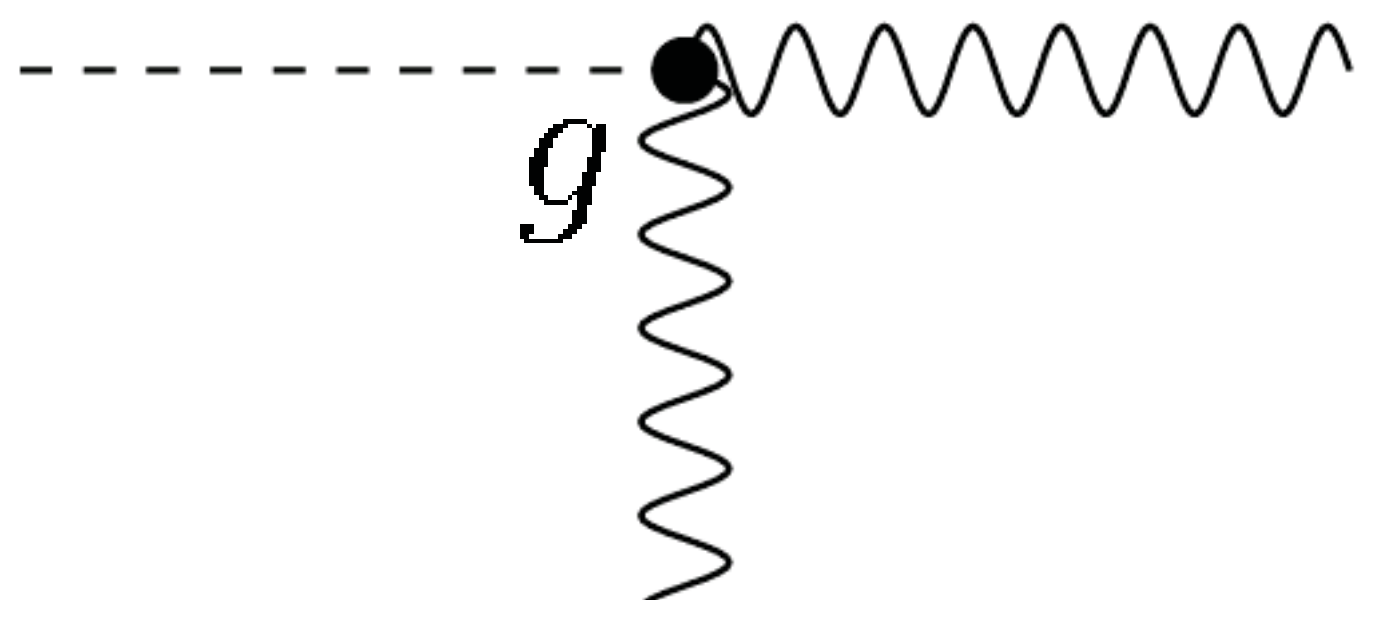




\section{Axion coupling to two photons}

- Effective higher dimensional coupling

$$
\mathcal{L}_{\text {Int }}=-\frac{1}{4} g a F^{\mu \nu} \tilde{F}_{\mu \nu}=-g a \mathbf{E} \cdot \mathbf{B}
$$

- Small coupling for large axion scale:

Small

$$
\sim g \sim \overline{2 \pi f_{a}}
$$




$$
\begin{aligned}
& \text { Large scale } \\
& \text { Small mass }
\end{aligned}
$$




\section{Axion See-Saw}

- The axion mass is small, too!

$$
m_{a} \sim \frac{m_{\pi} f_{\pi}}{f_{a}}
$$

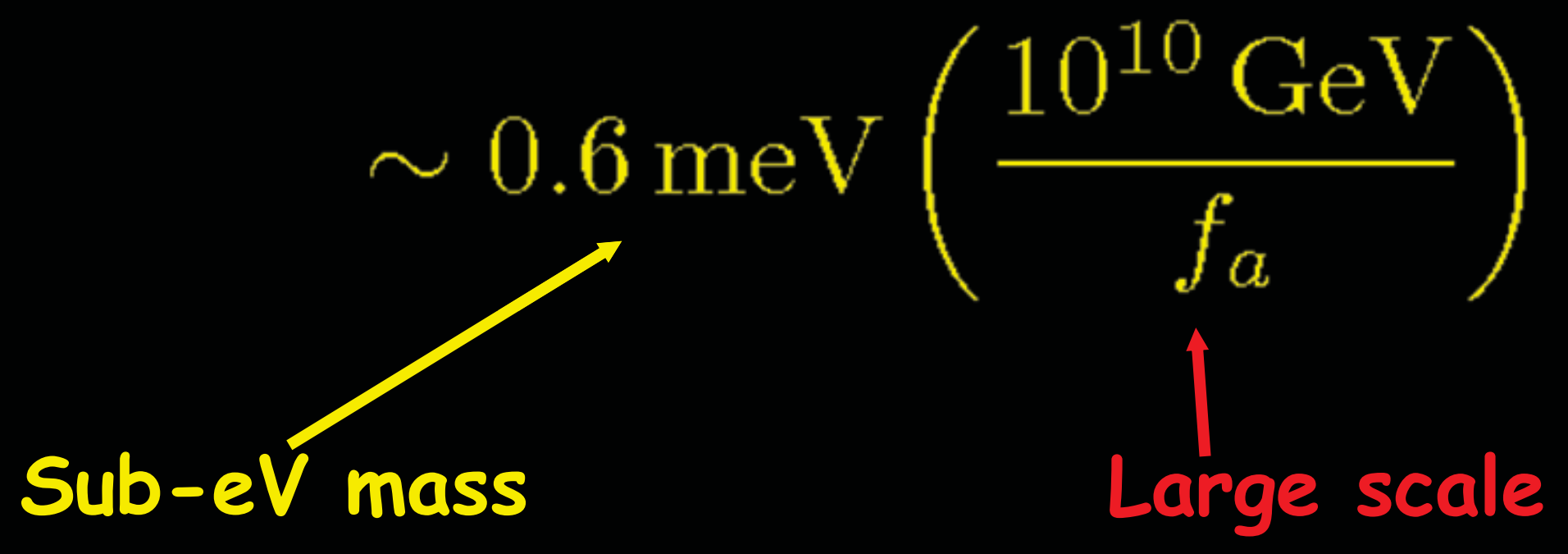


Axions live at small mass and coupling

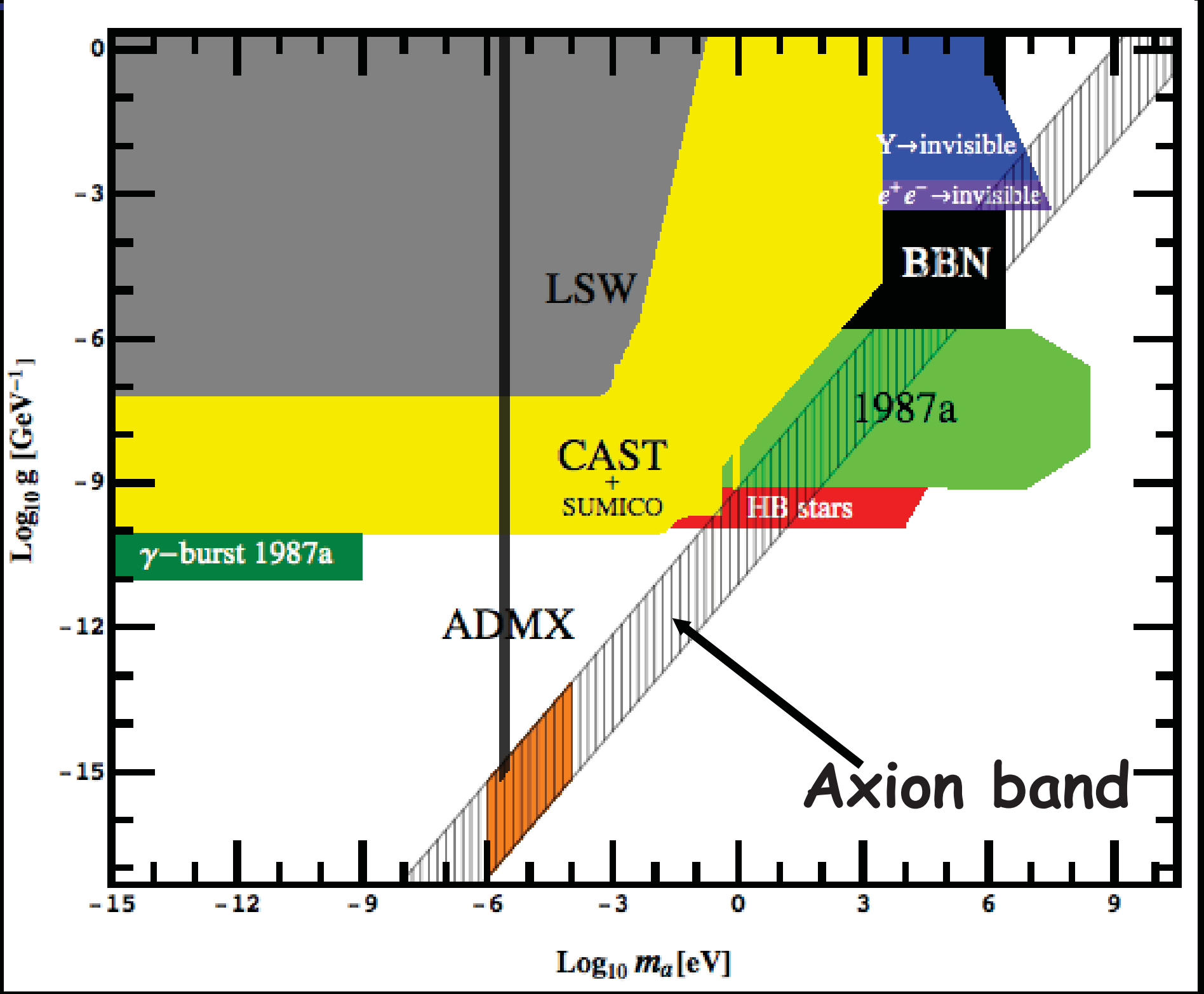




\section{The strong CP problem: Axions}

- Introduce new Peccei-Quinn symmetry to solve naturalness problem

- Predict as a consequence a new particle: The Axion

(it's a Weakly Interacting Sub-eV Particle)

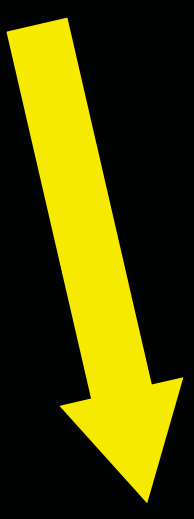

Good motivation

for axion/WISP experiments 
Axion Dark Matter 


\section{Axion production}

- $T<f_{a}$

- Axion potential is flat

- Axion can sit anywhere
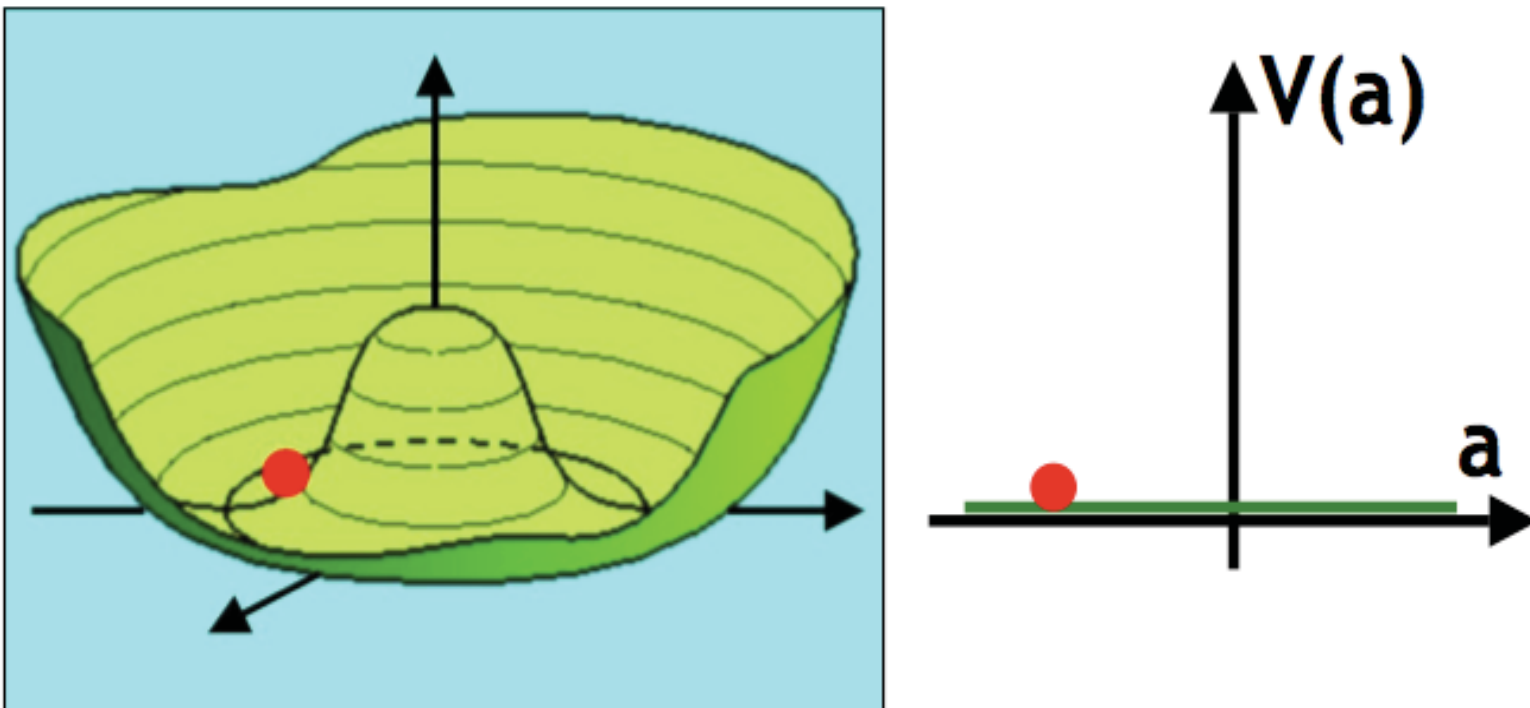

- $T<T_{Q C D}$

- Potential arises

- $H<m_{a}$

- Axion starts to oscillate
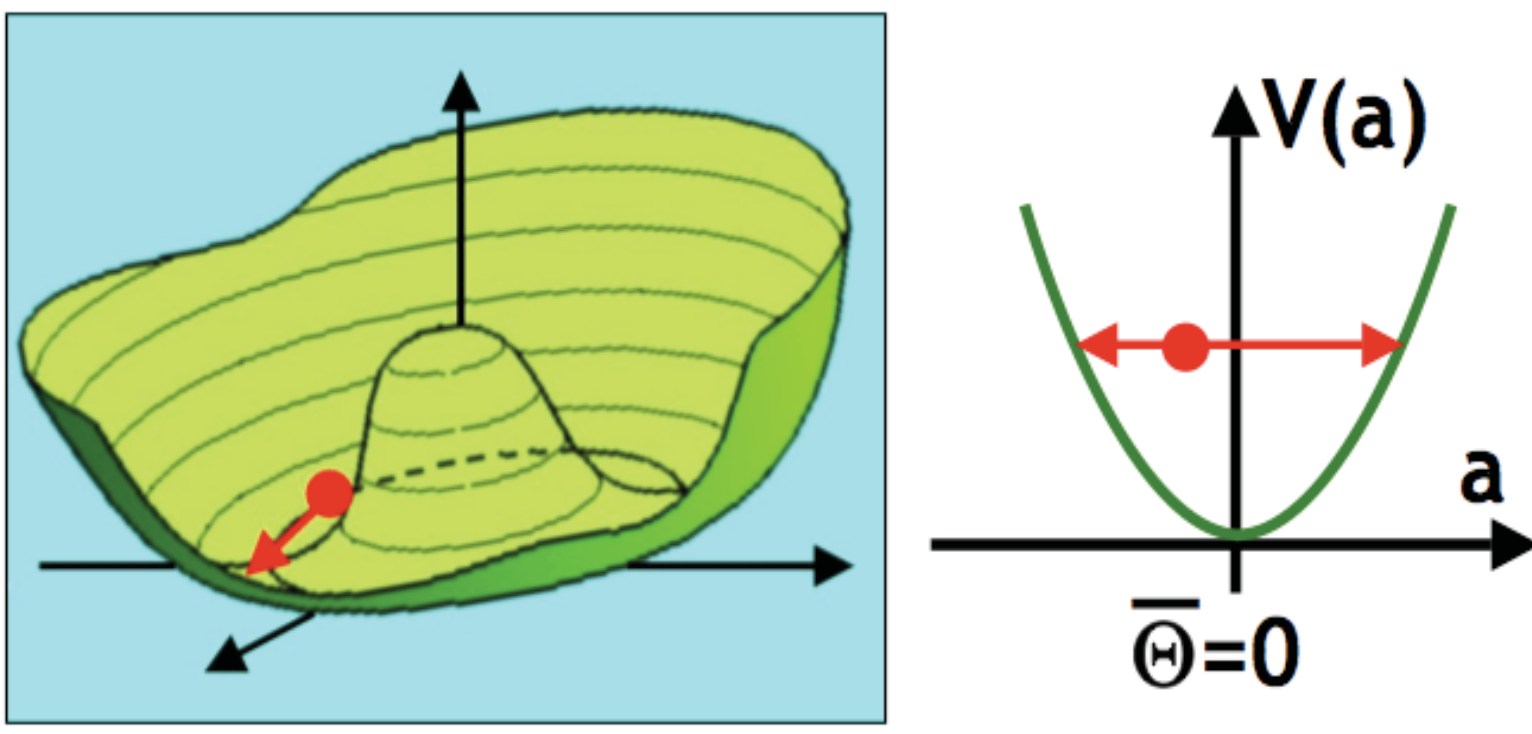

see S. Hannestad at Patras 2009 


\section{Oscillations behave like dark matter}

- Initial energy density

$$
\rho_{\text {ini }}=\frac{1}{2} m_{a}^{2} f_{a}^{2} \theta_{\text {ini }}^{2}
$$

+ damped Oscillations

$$
\ddot{\theta}+3 H \dot{\theta}+m_{a}^{2} \theta
$$

\section{Scales like matter}

$$
\rho_{a}(t) \sim \frac{\rho_{\mathrm{ini}}}{a^{3}(t)}
$$




\section{How much?}

- Energy density

$$
\Omega_{a} h^{2}=\kappa_{a}\left(\frac{f_{a}}{10^{12} \mathrm{GeV}}\right)^{1.175} \theta_{\mathrm{ini}}^{2}
$$




\section{Too much?}

- Energy density

$$
\begin{gathered}
\Omega_{a} h^{2}=\kappa_{a}\left(\frac{f_{a}}{10^{12} \mathrm{GeV}}\right)^{1.175} \theta_{\mathrm{ini}}^{2} \\
\text { For } \mathrm{f}_{\mathbf{a}}>\mathbf{1 0} \mathbf{0}^{12} \mathrm{GeV} \text { too much DM! }
\end{gathered}
$$


Axion dark matter

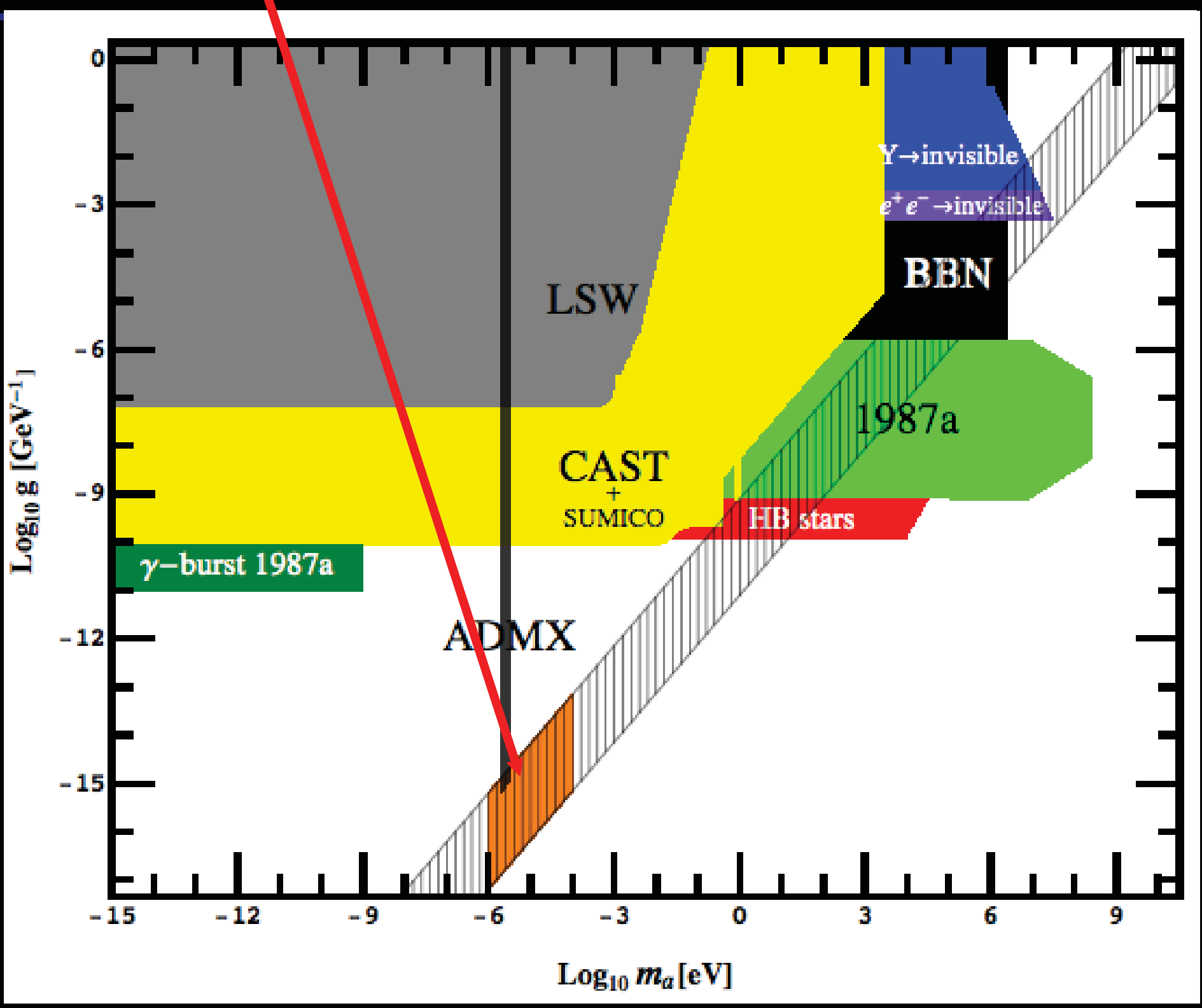


- Axions are very light:

- How can they be COLD dark matter?

\section{Non-thermal production!}




\section{Supercold?}

- At the beginning axion value everywhere the same

- Coherent Oscillations => Supercold!

$$
\begin{aligned}
& =p_{\text {ini }} \frac{T_{\text {today }}}{T_{\text {ini }}} \\
& \sim H_{\text {ini }} \frac{T_{\text {today }}}{T_{\text {ini }}}
\end{aligned}
$$

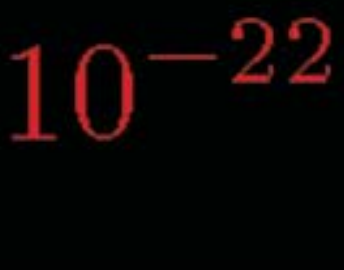




\section{The strong CP problem: Axions}

- Introduce new Peccei-Quinn symmetry to solve naturalness problem

- Predict as a consequence a new particle: The Axion

(it's a Weakly Interacting Sub-eV Particle)
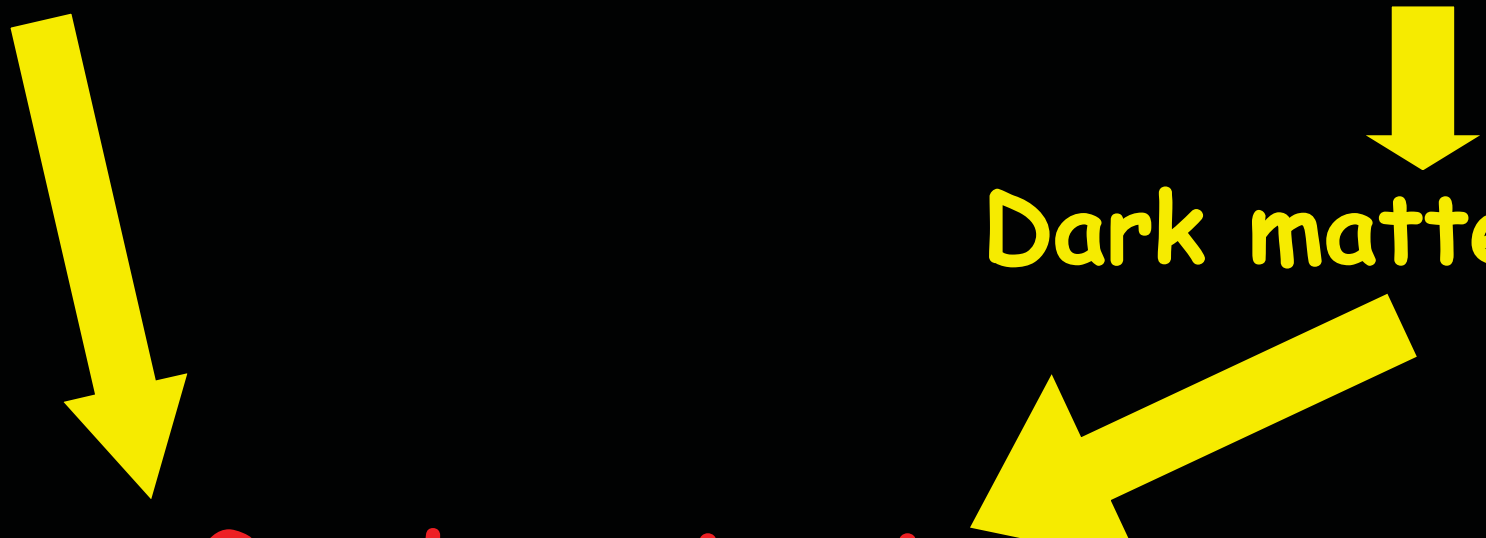

Dark matter candidate

for axion/WISP experiments 
"Light shining through a wall"

Laser
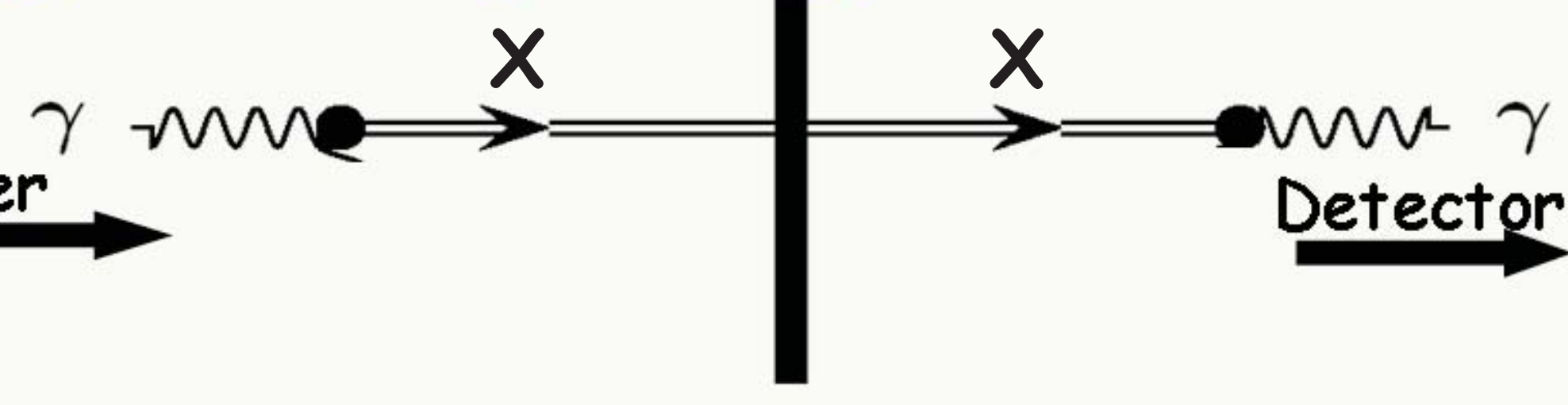
"Light shining through a wall"

Laser

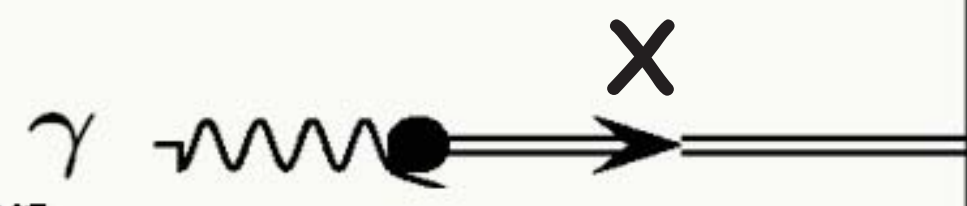

$10^{20} / \mathrm{s}$

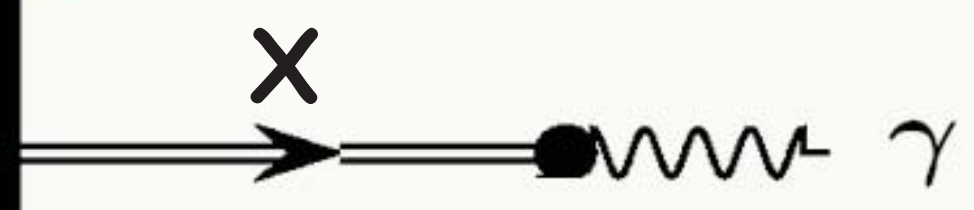

Detector

- Test $P_{\gamma \rightarrow X \rightarrow \gamma} \lesssim 10^{-20}$

- Enormous precision!

- Study extremely weak couplings! 


\section{Photons coming through the wall!}

- It could be Axion(-like particle)s!

- Coupling to two photons: $\frac{1}{M} a \tilde{F} F \sim \frac{1}{M} a \overrightarrow{\mathbf{E}} \cdot \overrightarrow{\mathbf{B}}$
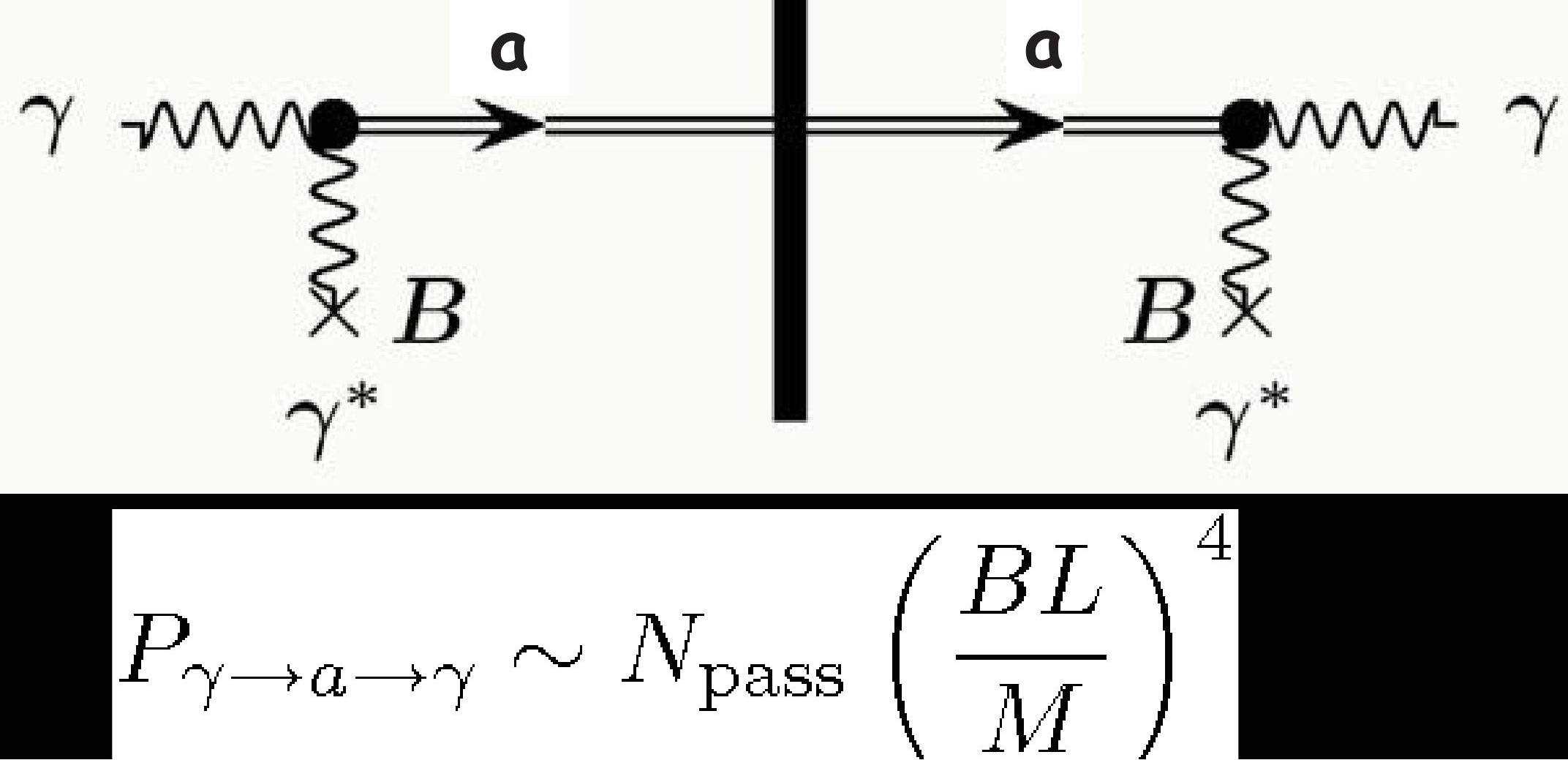


\section{Light Shining Through Walls}

- A lot of activity

- ALPS

- BMV

- Gamme $25 \mathrm{~cm}$

- LIPPS

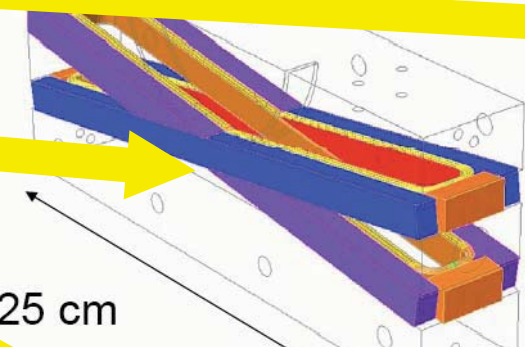

- OSQAR

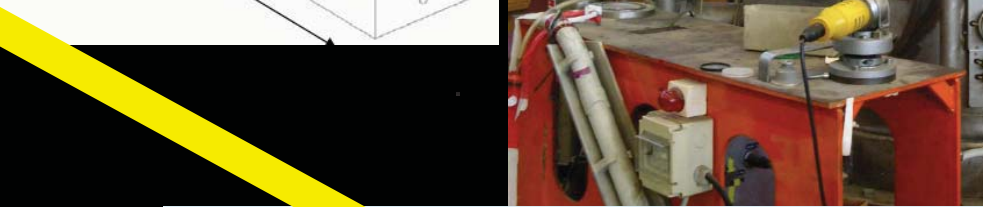

$(10)^{2}$
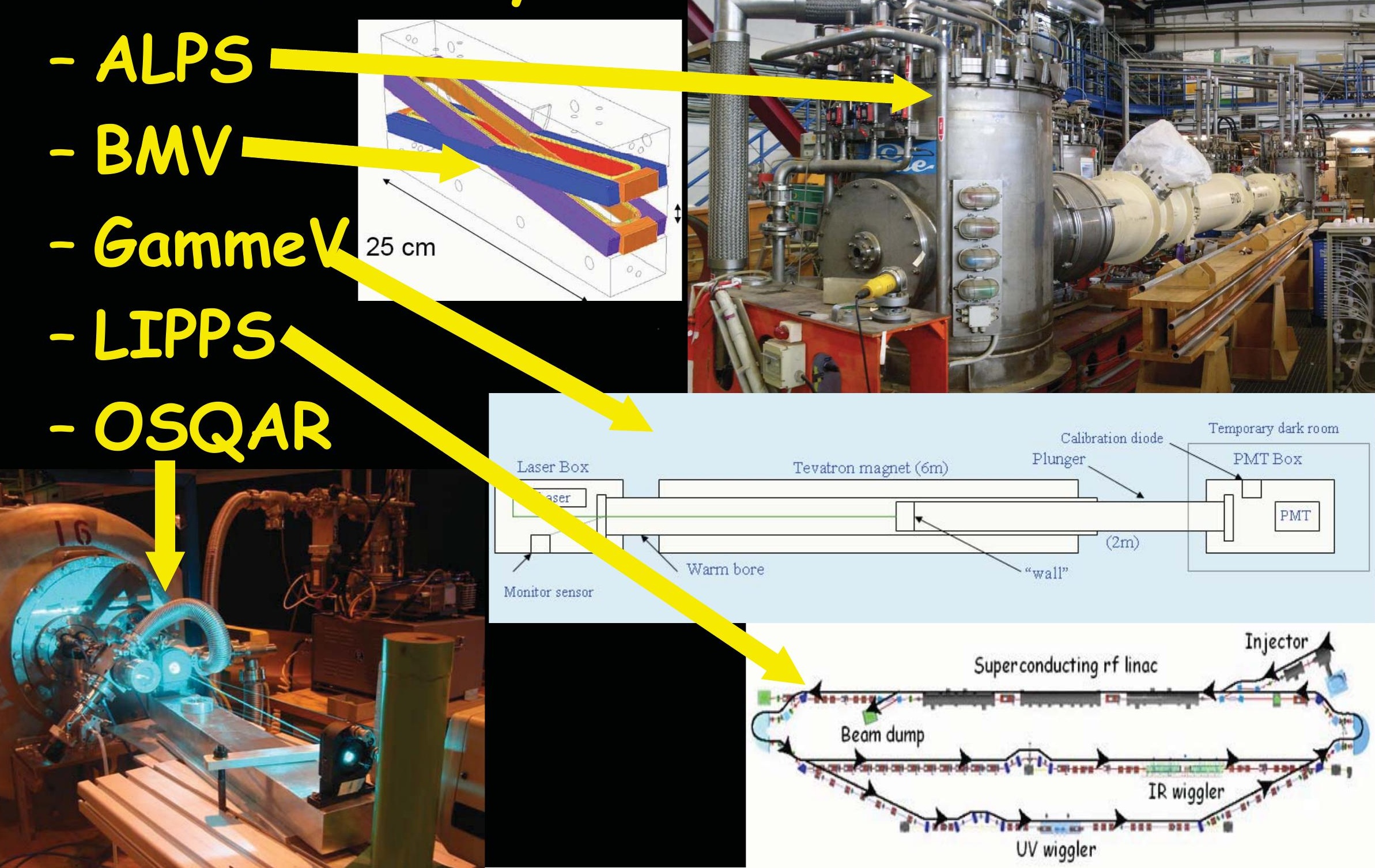


\section{Small coupling, small mass}

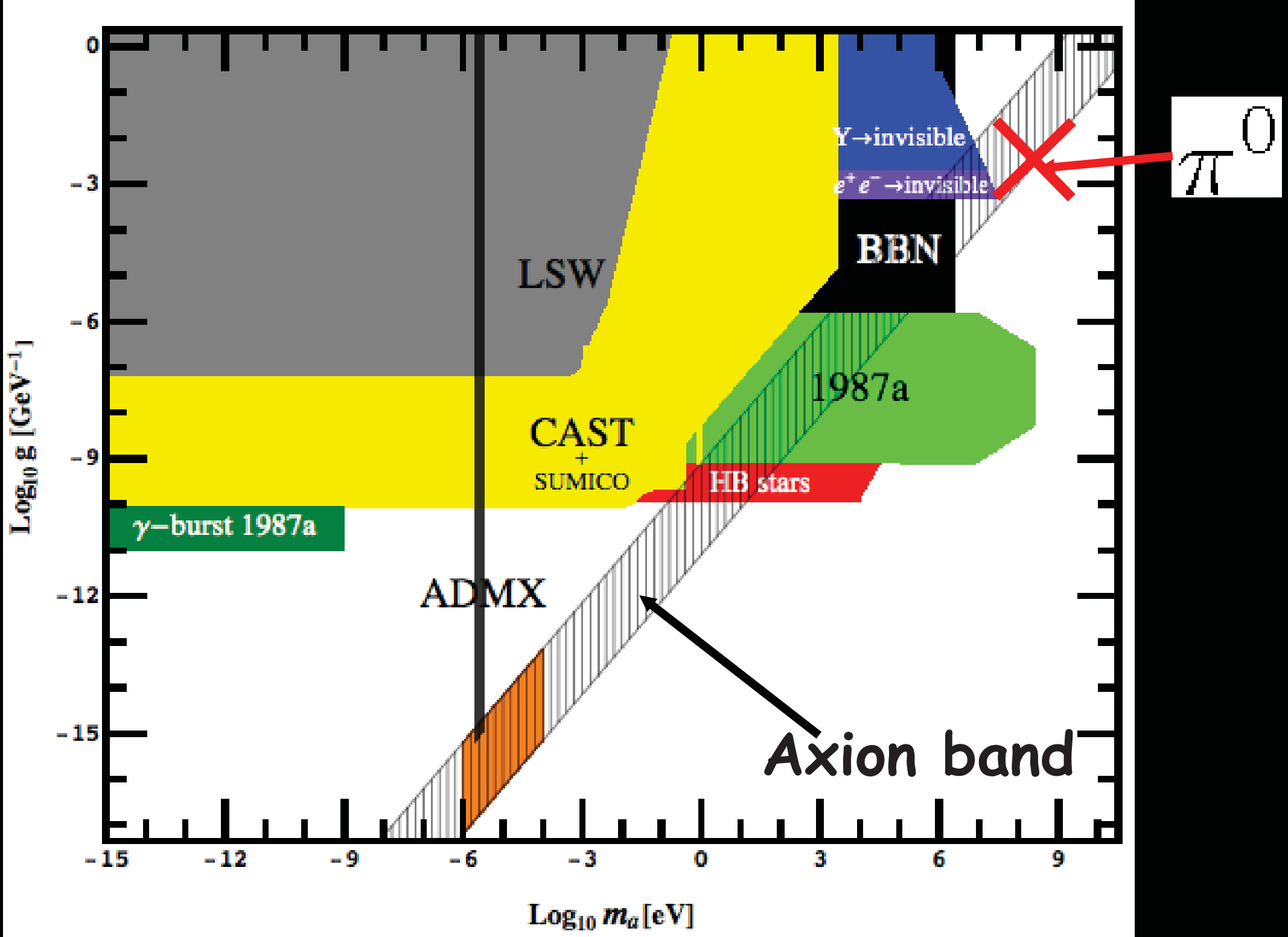


Hints for new Physics

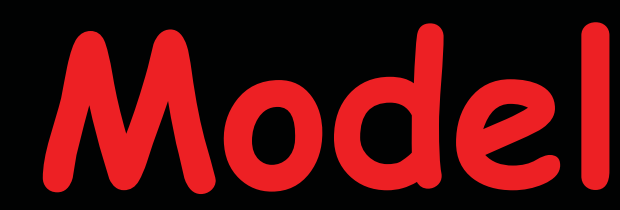

Building

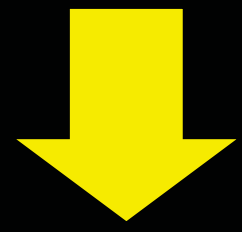

Bottom-up (pheno)

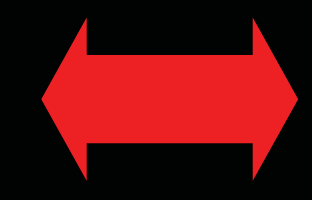

Top-down (theory)

Go back to drawing board 'Start from scratch' 


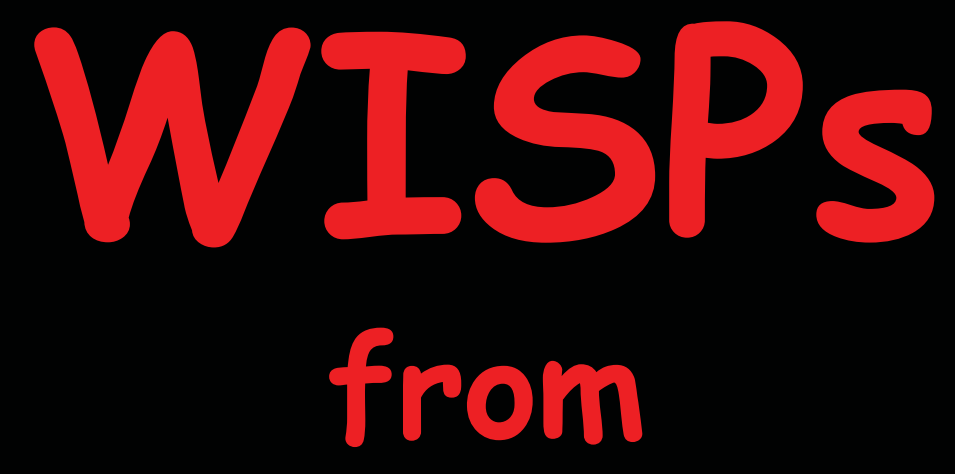

String Theory 


\section{String theory}

- Attempt to unify SM with gravity

- New concept: strings instead of point particles 
Axion(-like particles) 
String theory: Moduli and Axions

- String theory needs Extra Dimensions

Must compactify

- Shape and size deformations correspond to fields:

Moduli (WISPs) and Axions Connected to the fundamental scale, here string scale
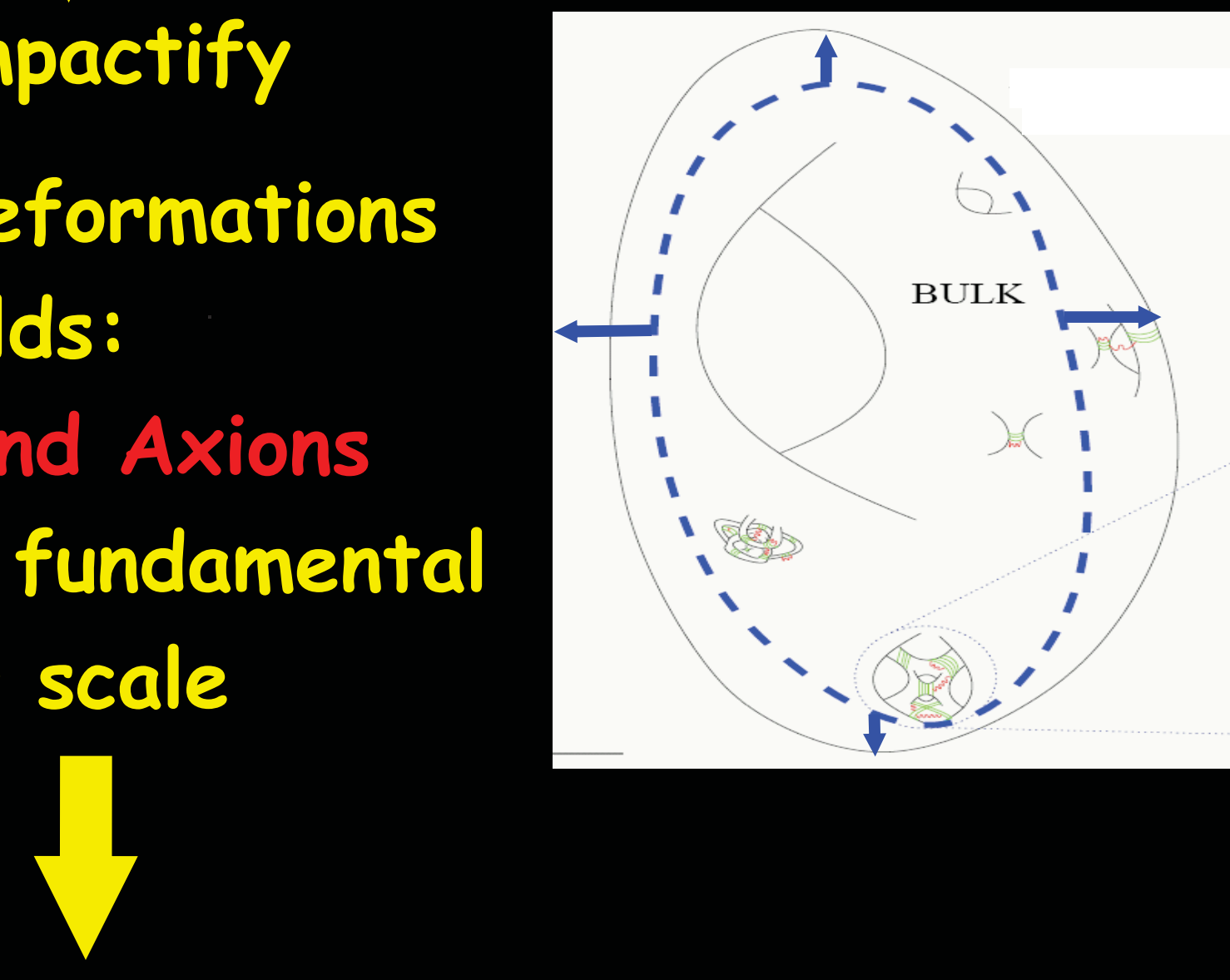


\section{Axions and Moduli}

- Gauge field terms

$$
\mathcal{L}=\frac{1}{g^{2}} F^{2}+i \theta F \tilde{F}
$$

+ Supersymmetry/supergravity

$$
\mathcal{L}=\operatorname{Re}[f(\Phi)] F^{2}+\operatorname{Im}[f(\Phi)] F \tilde{F}
$$

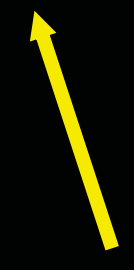

Scalar ALP/moduli coupling 


\section{Axions and Moduli}

- Gauge couplings always field dependent (no free coupling constants)

- Axions + Moduli always present in String theory 


\section{Masses and Couplings}

- "Axion scale" related to fundamental scale

$$
f_{a} \sim \frac{M_{P}}{\text { Volume }^{x}} \sim M_{s}\left(\frac{M_{s}}{M_{P}}\right)^{y}
$$

- If $Q C D$ axion: $m_{a}$ fixed

- However, if not QCD axion

$$
m_{\mathrm{ALP}} \sim \frac{\Lambda^{2}}{f_{a}} \quad \text { (nearly) arbitrary }
$$


Axion (like particles): Where are we?

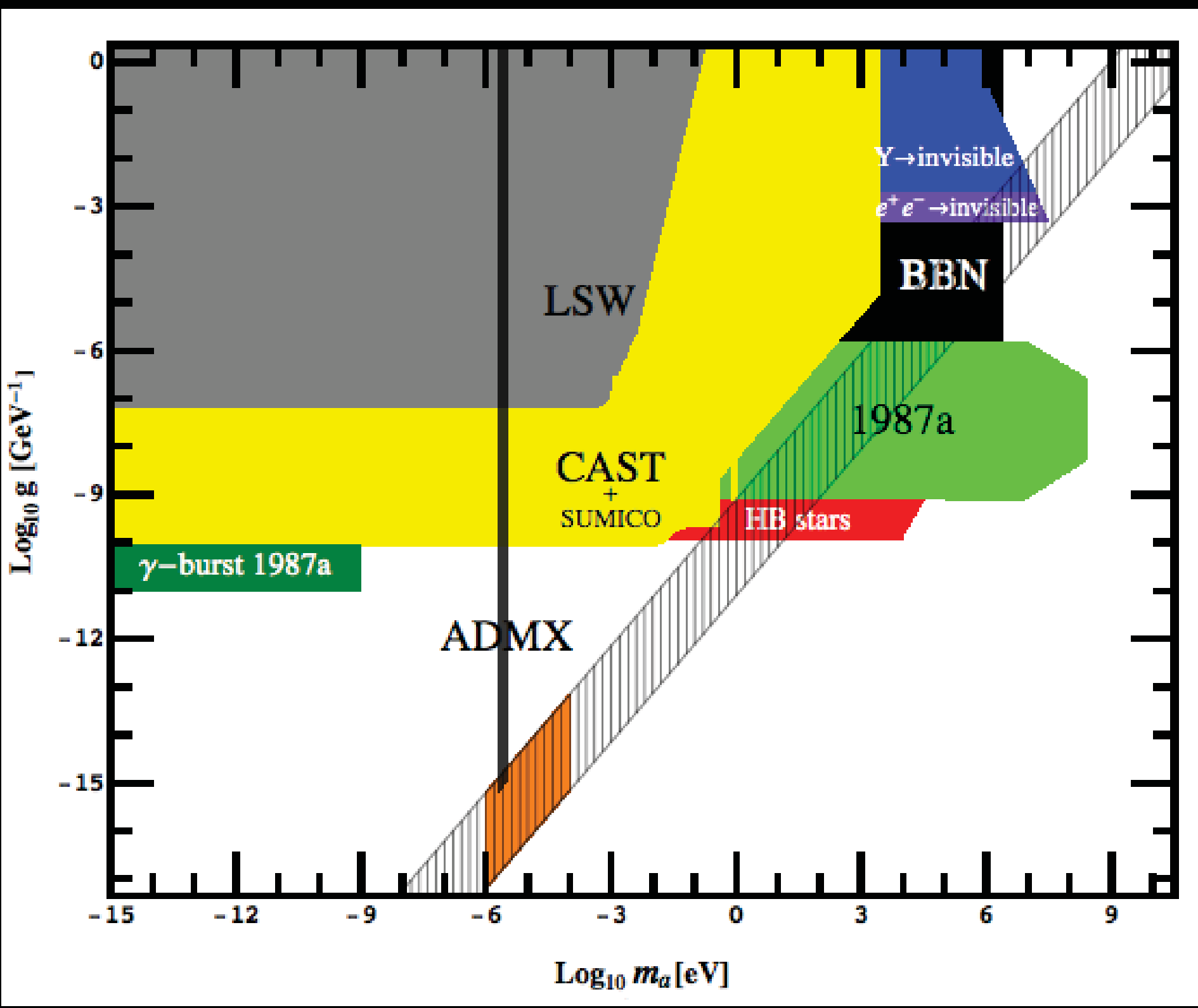


Axion (like particles): Where are we?

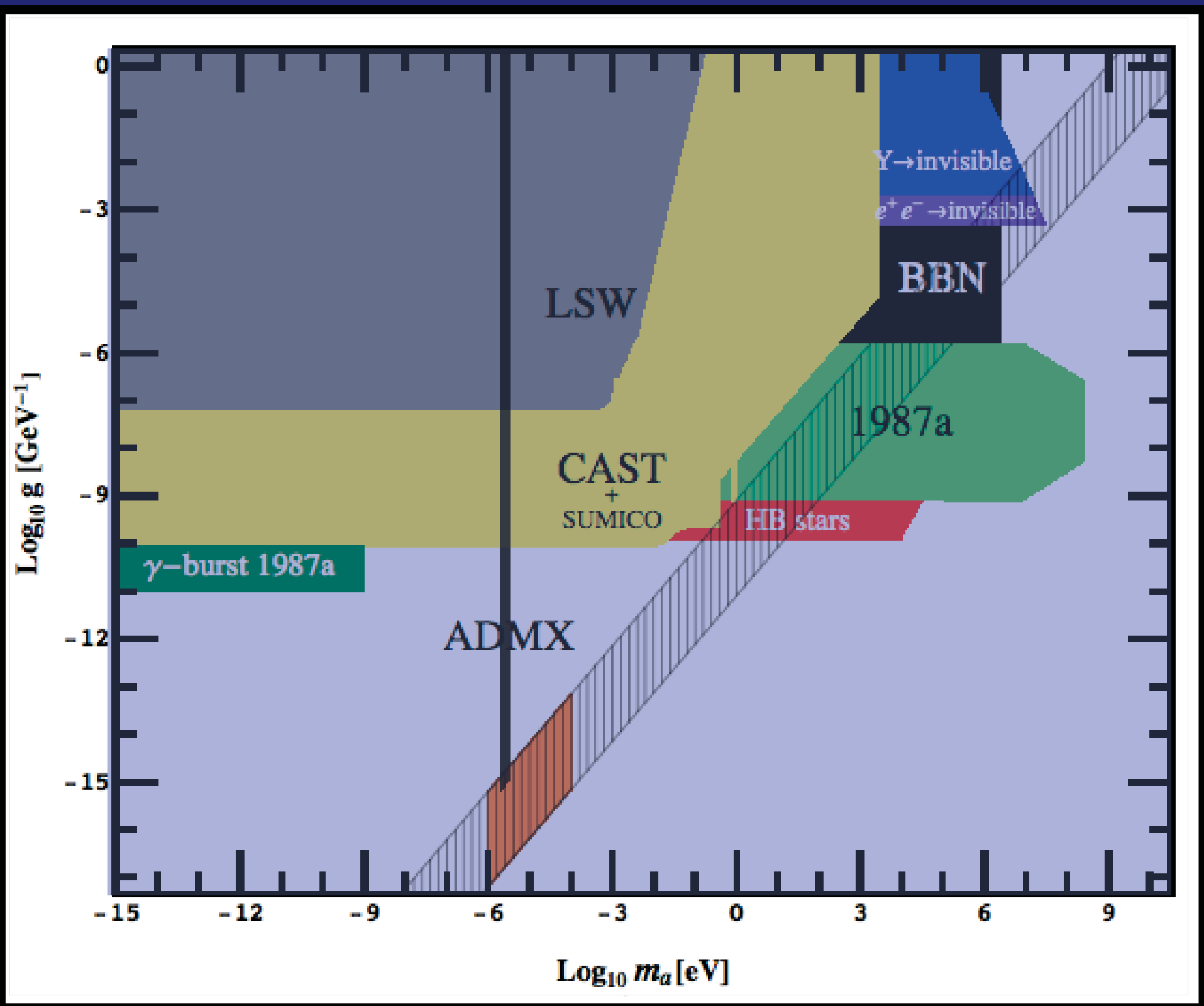


Hidden Photons 


\section{String theory likes extra gauge groups}

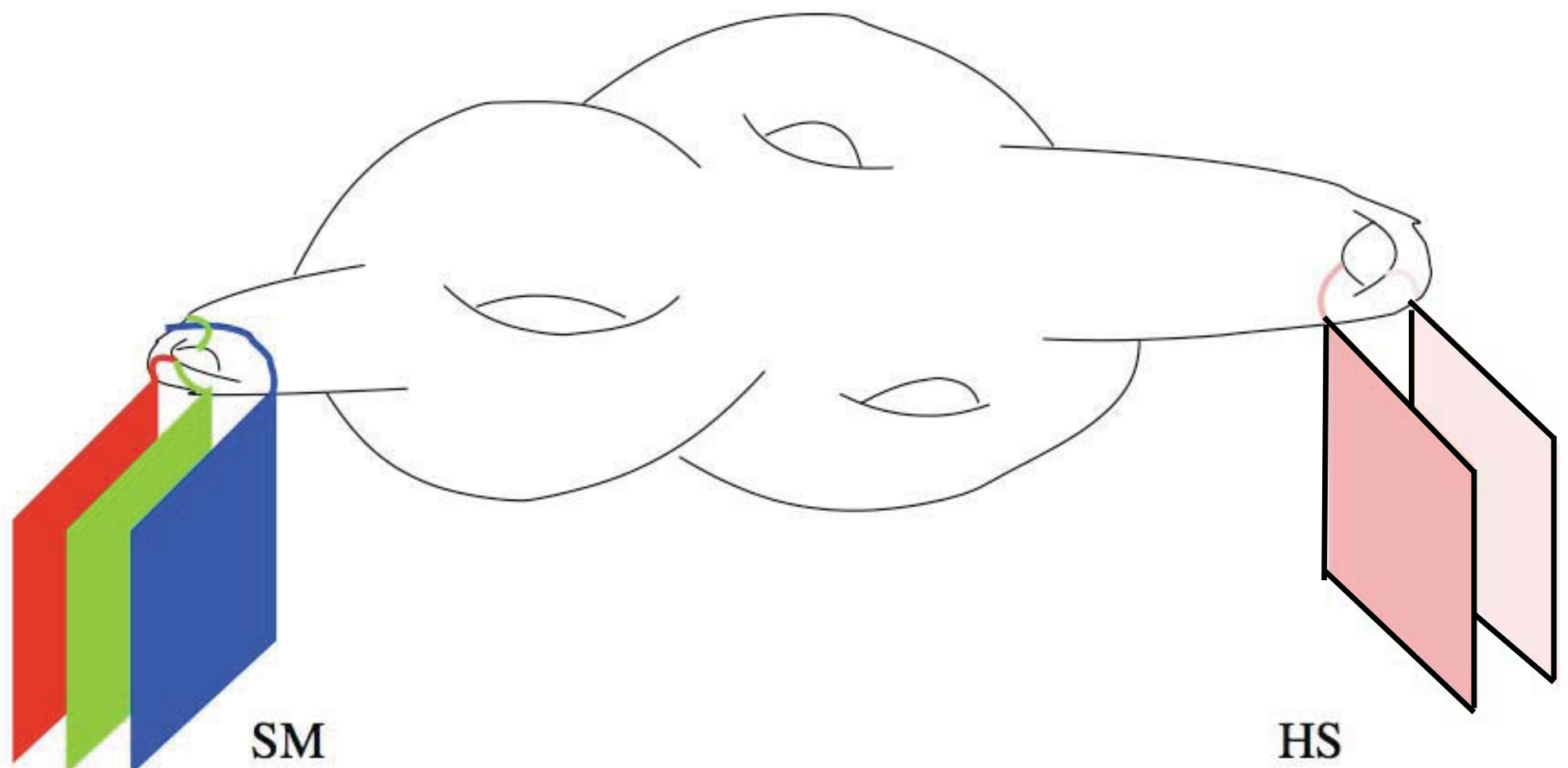

D. Luest 0707.2305

\section{$\mathrm{U}(\mathrm{A}) \times \mathrm{U}(\mathrm{B}) \times \mathrm{U}(\mathrm{C})$}

\section{Many extra U(1)s!}

Candidates for WISPs
S. Abel, JJ, V. Khoze, A. Ringwald hep-ph/0608248

S. Abel, M. Goodsell, JJ, V. Khoze,

A. Ringwald 0803.1449 


\section{How coupled?}

- Kinetic mixing

$\mathcal{L}_{\text {gauge }}=-\frac{1}{4} F_{(\mathrm{A})}^{\mu \nu} F_{(\mathrm{A}) \mu \nu}-\frac{1}{4} F_{(\mathrm{B})}^{\mu \nu} F_{(\mathrm{B}) \mu \nu}+\frac{\chi}{2} F_{(\mathrm{A})}^{\mu \nu} F_{(\mathrm{B}) \mu \nu}$,

\section{"Our" U(1) "Hidden" U(1)}

Mixing

+ Mass

$$
\mathcal{L}_{\text {mass }}=\frac{1}{2} m_{\gamma^{\prime}}^{2} X^{\mu} X_{\mu}
$$

photon - hidden photon oscillations

Light shining through walls

Fixed target signatures 
- Another simple motivation:

Question: Are there extra gauge groups?

Testcase: $U(1)$ is the simplest example we can think of! 


\section{How to get Kinetic Mixing ...}

- Field Theory:

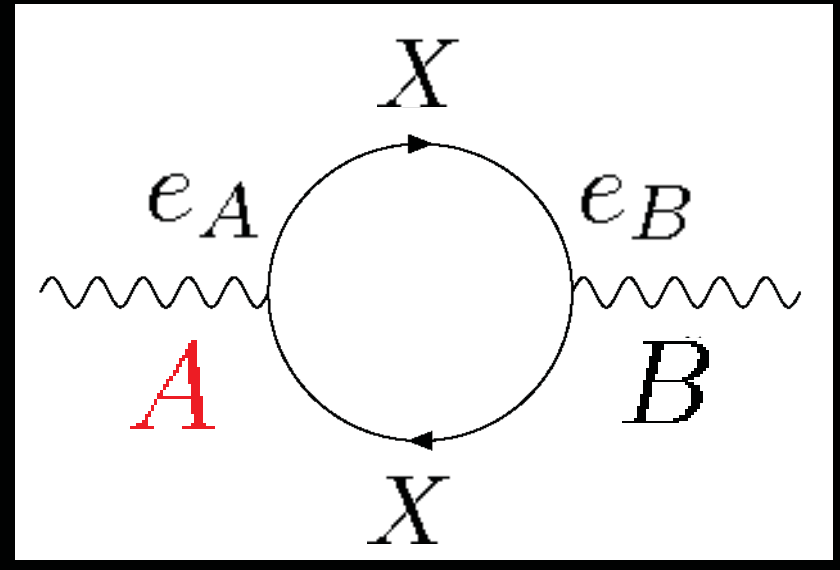

$\Rightarrow \chi \sim \frac{e_{A} e_{B}}{16 \pi^{2}} \log \left(\frac{m_{X}^{2}}{\Lambda^{2}}\right)$

UV sensitive since dimension 4 operator.

Tests underlying high scale physics! 


\section{How to get Kinetic Mixing ...}

- Field Theory:

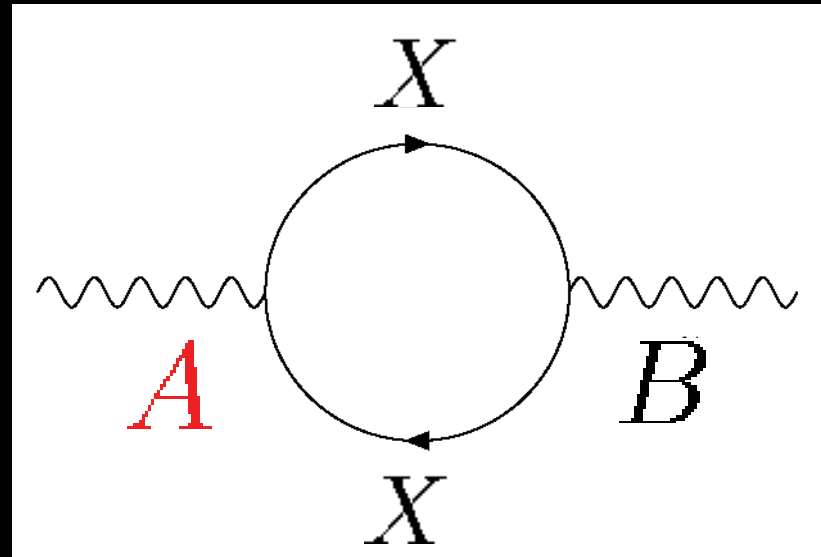

- String Theory:
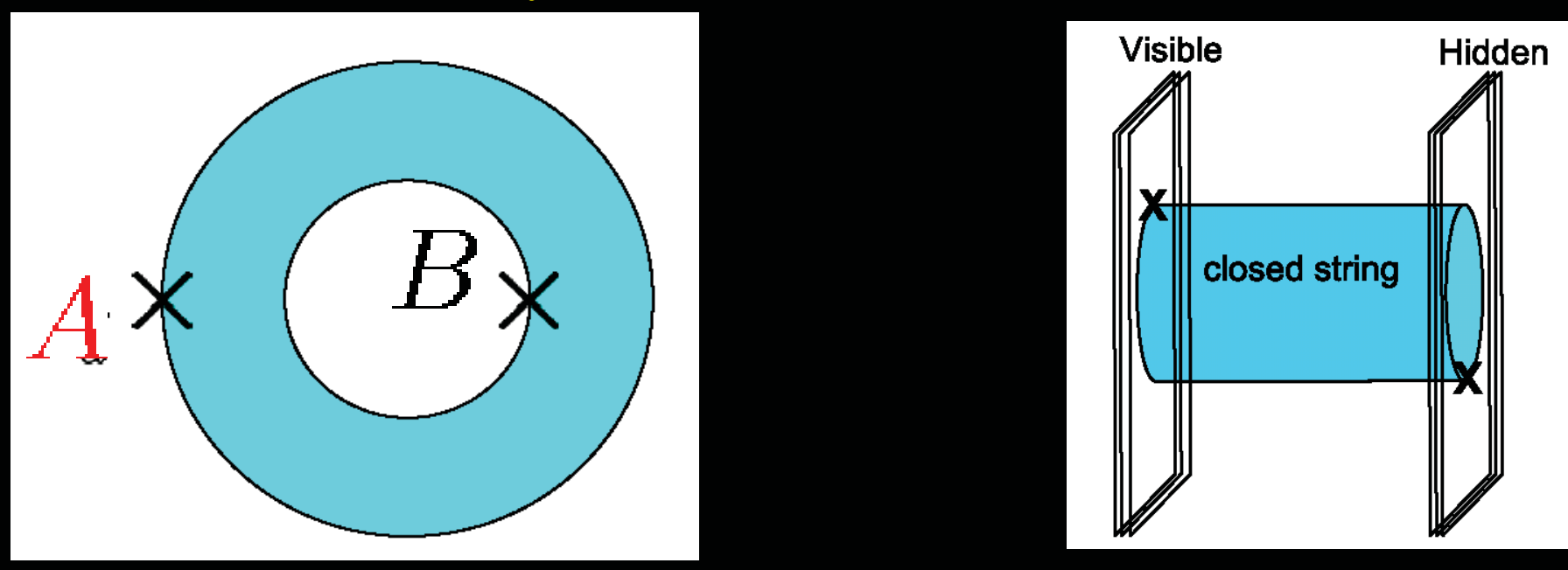


\section{Hidden by distance}

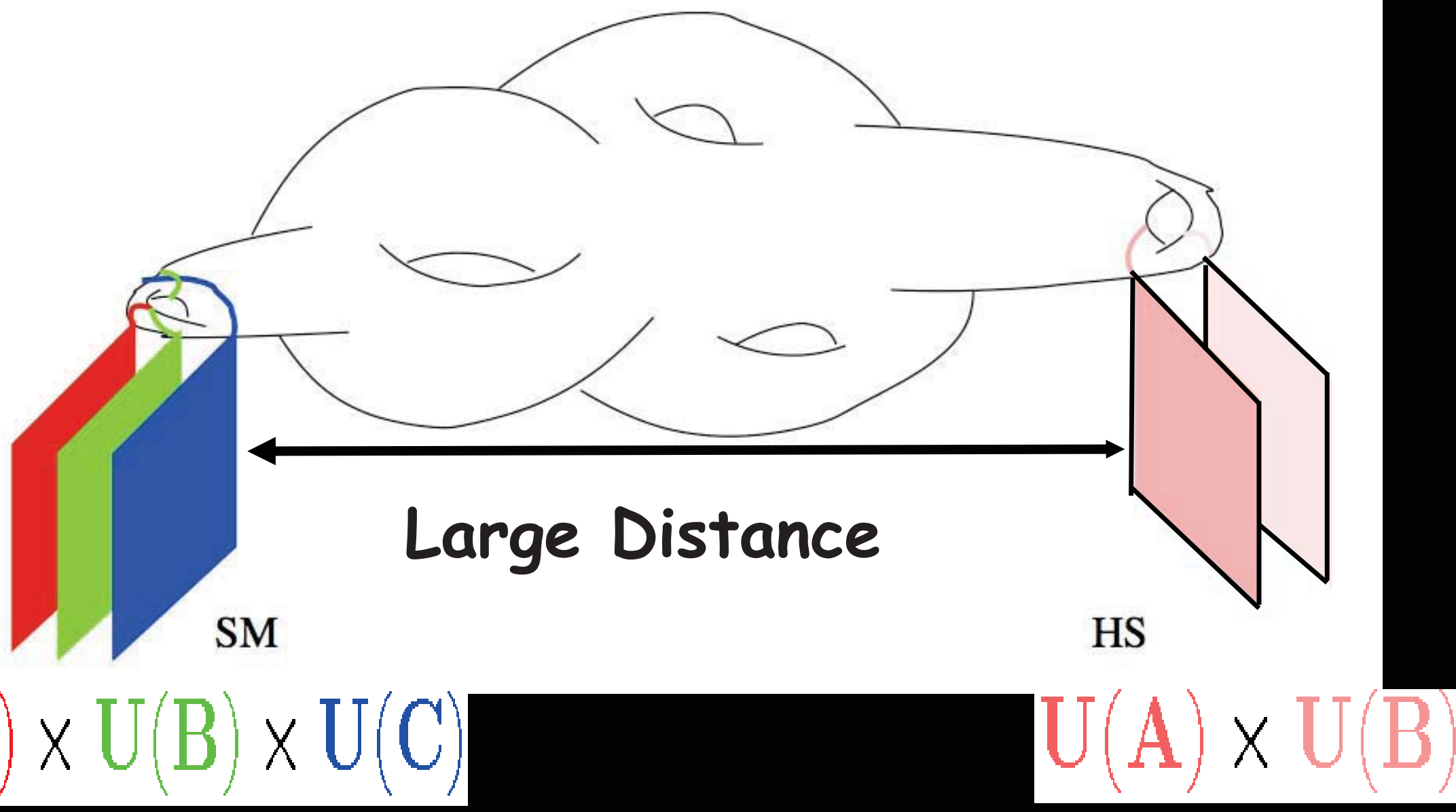

$$
\chi \sim \frac{g_{s}}{8 \pi} \frac{1}{\text { Volume }^{x}}
$$




\section{Hidden by weakness}

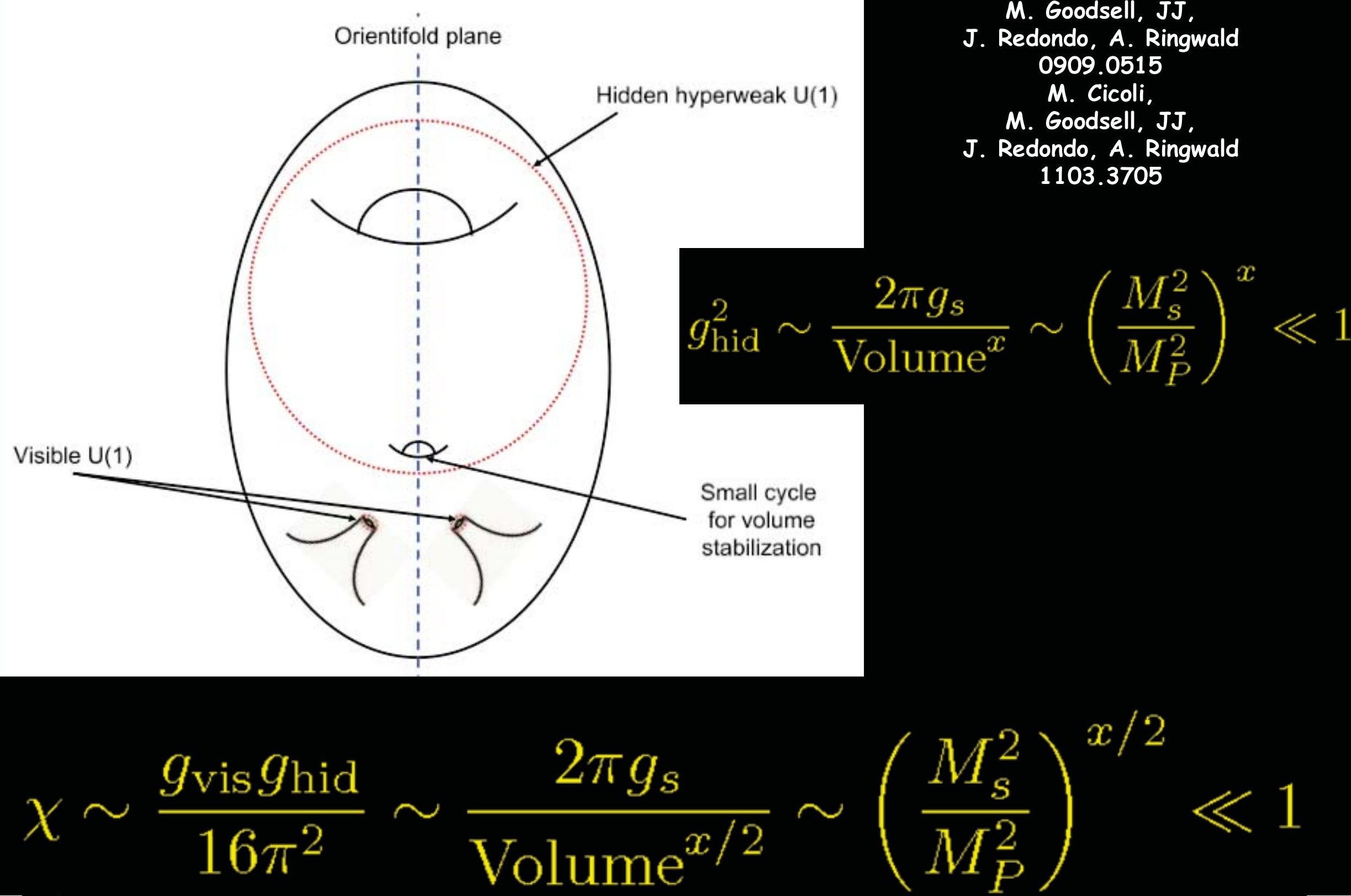




\section{Hidden Photons, all over the place}

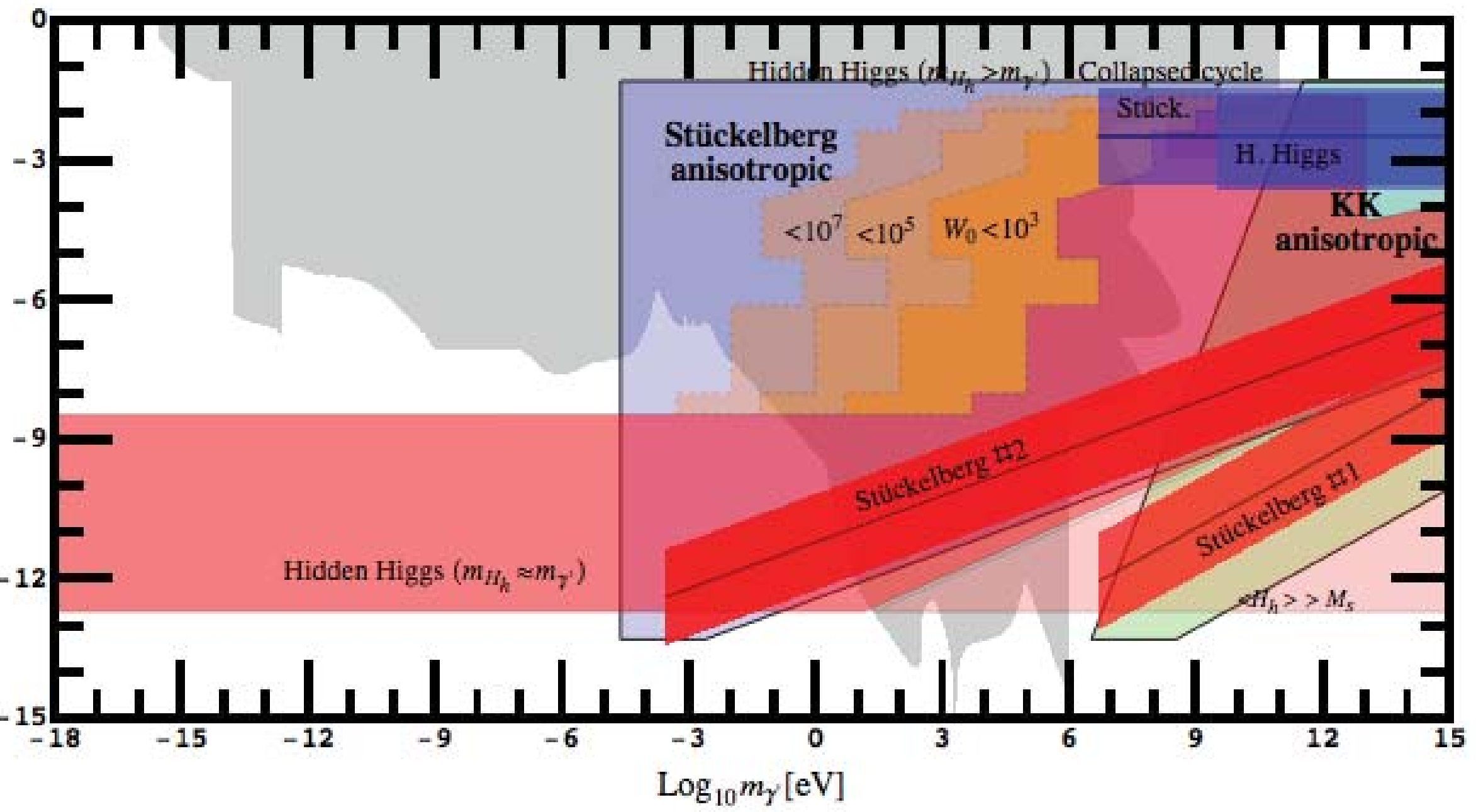




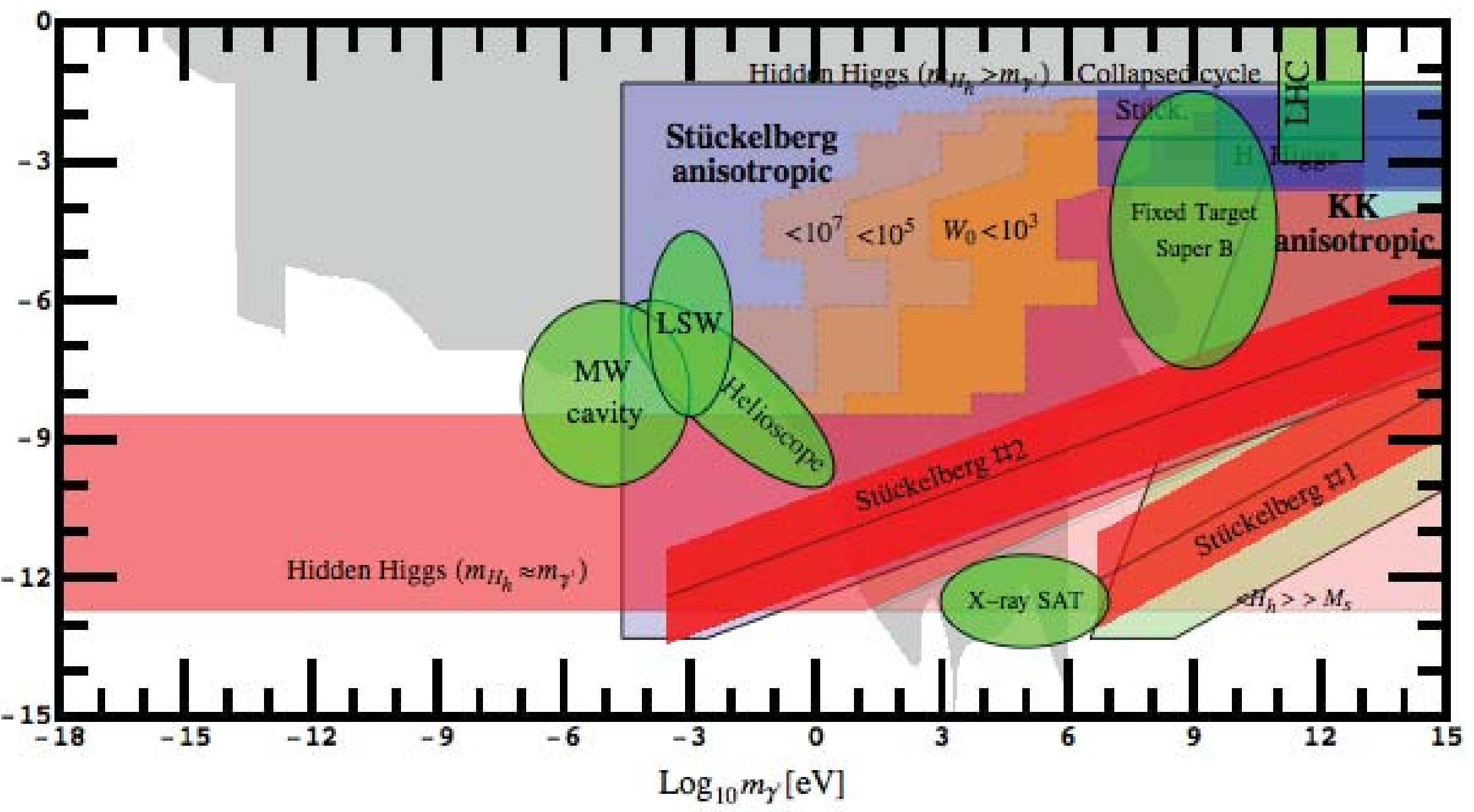




\section{Hidden Matter}

Minicharged Particles 


\section{Minicharged particles}

- Extra U(1) gauge boson, i.e. extra photon

- Normal matter not charged under it

- Main interaction:

- Small coupling to photons, related to kinetic mixing

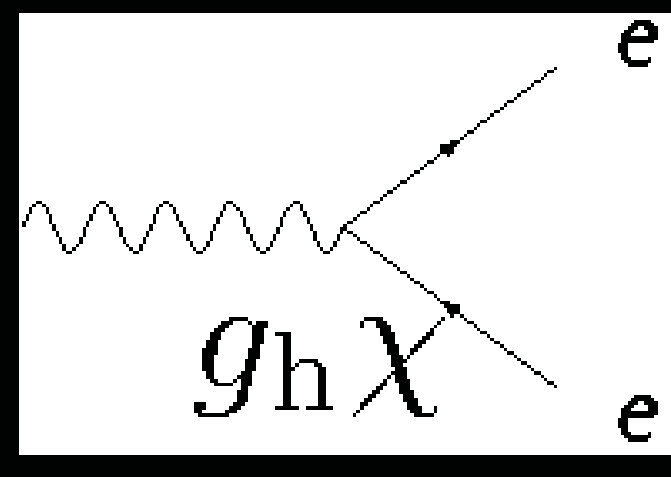

$\chi \sim$ dimensionless $\ll 1$

$g_{\mathrm{h}} \sim$ dimensionless $\sim 1$ or $\ll 1$ 


\section{How coupled?}

- Kinetic mixing

$\mathcal{L}_{\text {gauge }}=-\frac{1}{4} F_{(\mathrm{A})}^{\mu \nu} F_{(\mathrm{A}) \mu \nu}-\frac{1}{4} F_{(\mathrm{B})}^{\mu \nu} F_{(\mathrm{B}) \mu \nu}+\frac{\chi}{2} F_{(\mathrm{A})}^{\mu \nu} F_{(\mathrm{B}) \mu \nu}$,

\section{„Our" U(1) „Hidden" U(1)}

Mixing

+ Matter $\quad \mathcal{L}_{\text {int }}=g_{\text {hid }} \bar{h} \gamma_{\mu} X^{\mu} h$

Particles with small electric charges

LSW + other experiments 


\section{Kinetic Mixing - How to get Minicharges}

- Two U(1)'s

$\mathcal{L}_{\text {gauge }}=-\frac{1}{4} F_{(\mathrm{A})}^{\mu \nu} F_{(\mathrm{A}) \mu \nu}-\frac{1}{4} F_{(\mathrm{B})}^{\mu \nu} F_{(\mathrm{B}) \mu \nu}+\frac{\chi}{2} F_{(\mathrm{A})}^{\mu \nu} F_{(\mathrm{B}) \mu \nu}$,

\section{"Our" U(1) "Hidden" U(1)}

\section{Mixing}

$\begin{aligned} & =" A^{2}+B^{2}-2 \chi A B \\ & =A^{2}+(B+\chi A)^{2}+\mathcal{O}\left(\chi^{2}\right)\end{aligned}$

Diagonalization: $B^{\mu} \rightarrow B^{\mu}+\chi A^{\mu}$ 


\section{Kinetic Mixing - How to get Minicharges}

- Two U(1)'s

$\mathcal{L}_{\text {gauge }}=-\frac{1}{4} F_{(\mathrm{A})}^{\mu \nu} F_{(\mathrm{A}) \mu \nu}-\frac{1}{4} F_{(\mathrm{B})}^{\mu \nu} F_{(\mathrm{B}) \mu \nu}+\frac{\chi}{2} F_{(\mathrm{A})}^{\mu \nu} F_{(\mathrm{B}) \mu \nu}$,

\section{"Our" U(1) "Hidden" U(1)}

Mixing

Diagonalization: $B^{\mu} \rightarrow B^{\mu}+\chi A^{\mu}$

$g_{h} \overline{\mathbf{h}} B^{\mu} \mathbf{h} \rightarrow g_{h} \overline{\mathbf{h}} B^{\mu} \mathbf{h}+\chi g_{h} \overline{\mathbf{h}} A^{\mu}$ 
Necessary ingredients:

- Extra 'hidden' U(1) gauge groups!

- Kinetic mixing term!

- Matter charged under hidden U(1) 


\section{String theory likes extra matter}

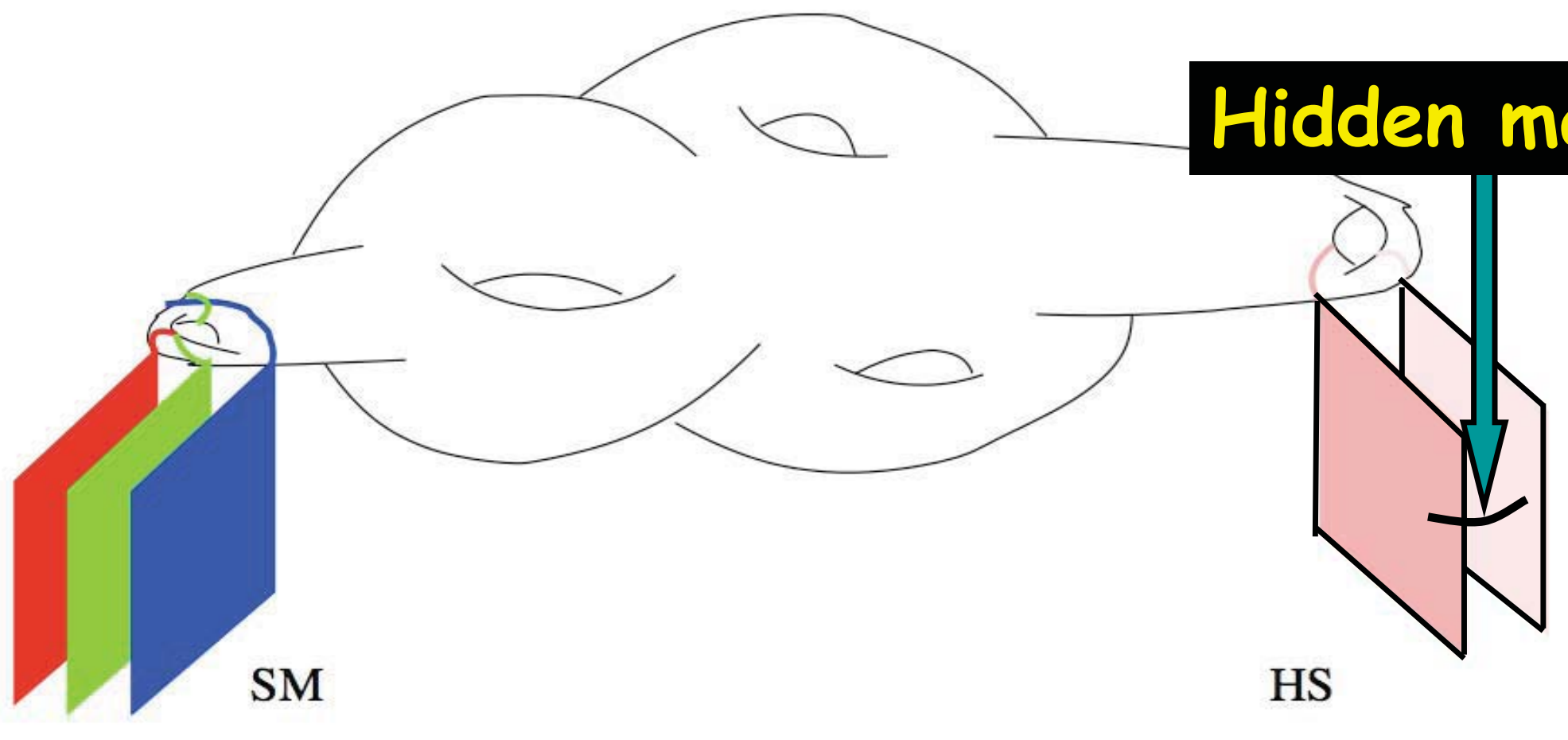

$\mathrm{U}(\mathrm{A}) \times \mathrm{U}(\mathrm{B}) \times \mathrm{U}(\mathrm{C})$

\section{Hidden sector matter}

Appears to be minicharged 


\section{Minicharged particles...}

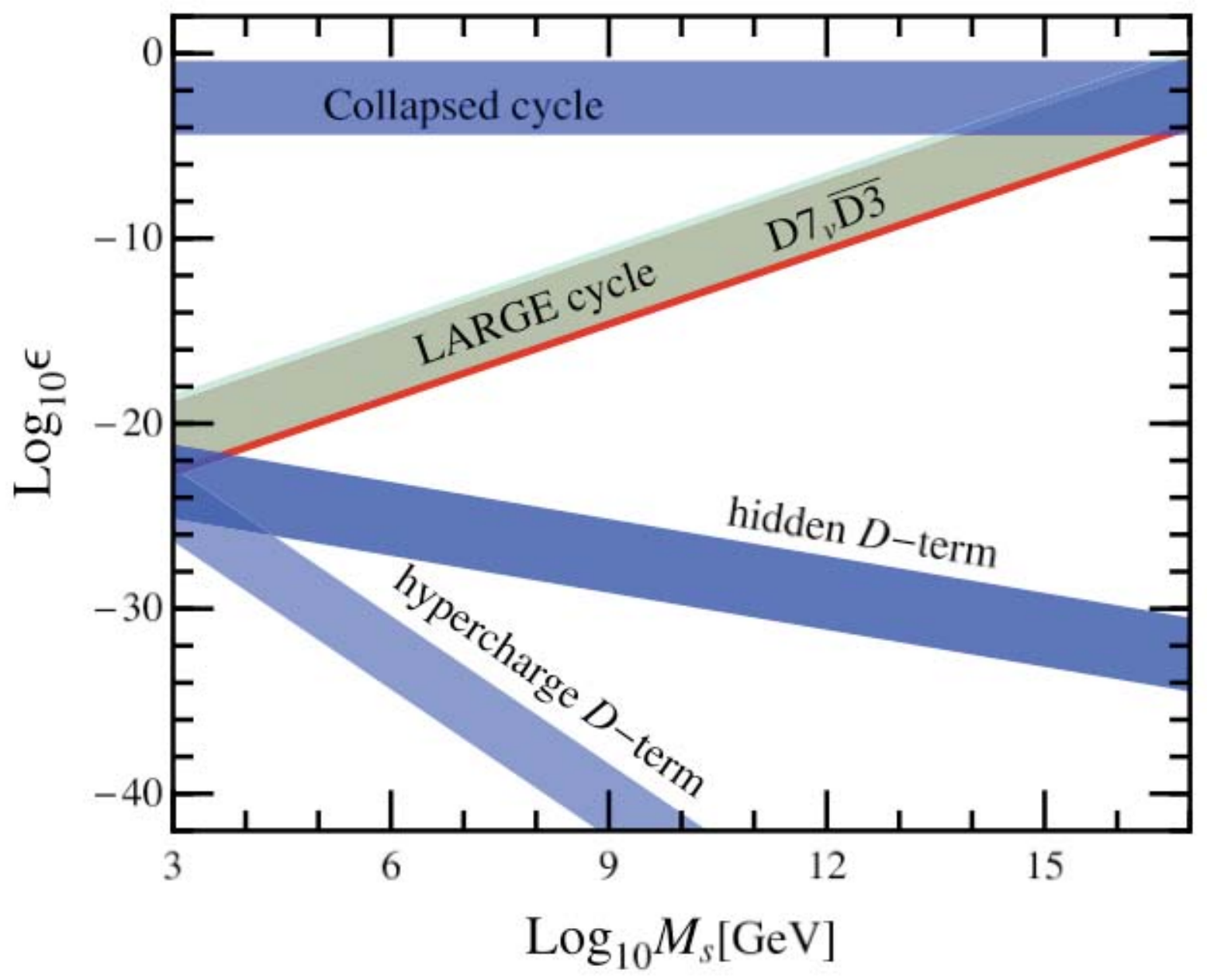




$$
\text { Crazy stuff }
$$


Summary 


\section{Summary}

- Good motivation for:

Axions and other light very weakly coupled

From Bottom-Up (Strong CP problem, DM)

Top-down (String theory)

+++ Cool experiments

- Insight into light particles may provide information on hidden sectors and thereby into the underlying fundamental theory

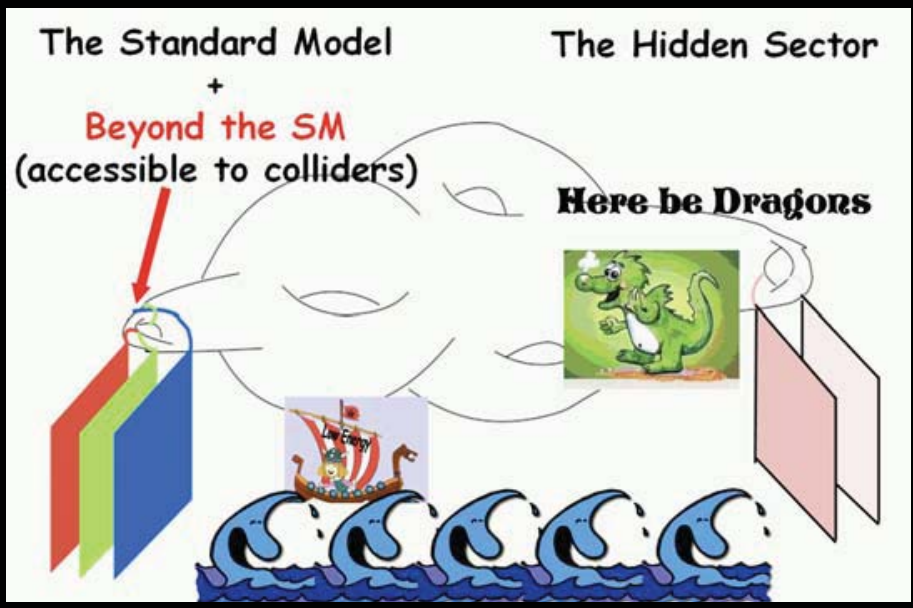

- Surprises like Lorentz symmetry violation possible! explore 'The Low Energy Frontier' 


$$
\begin{aligned}
& 2 . \\
& \text { Light particles in } \\
& \text { astrophysics and } \\
& \text { cosmology }
\end{aligned}
$$


Hints for new Physics

Model

Building

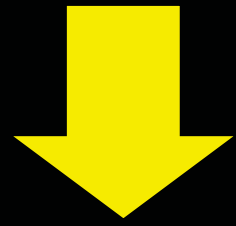

Bottom-up

(pheno)
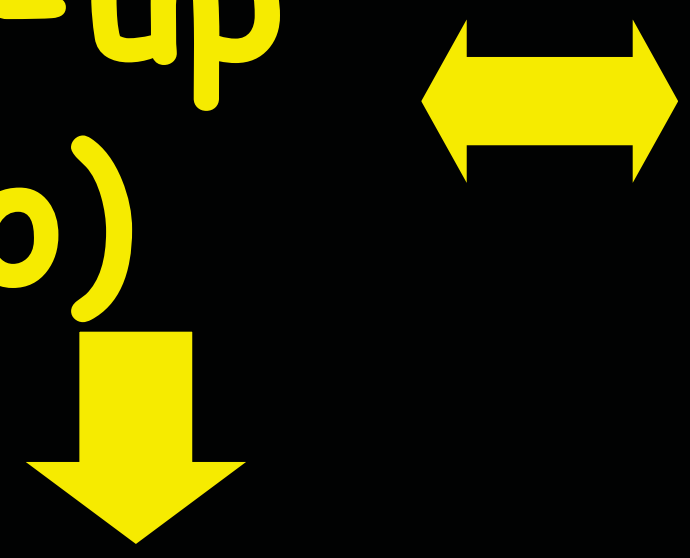

Top-down
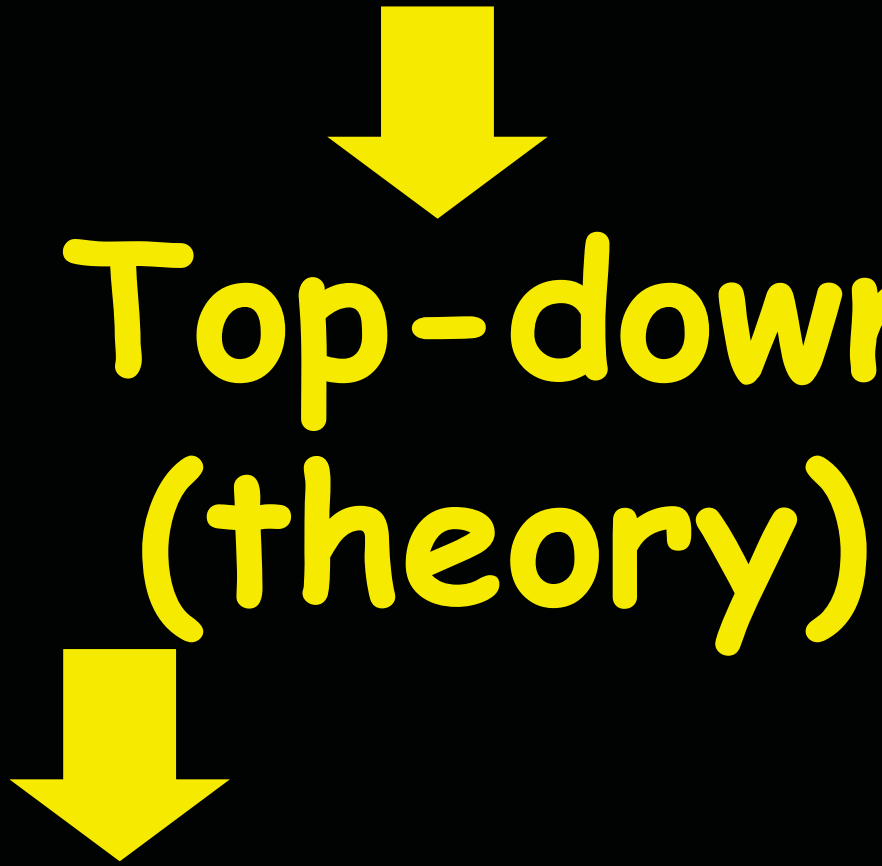


$$
\begin{gathered}
\text { Warmup } \\
\text { More particles + } \\
\text { Photon- WISP } \\
\text { interactions }
\end{gathered}
$$




\section{Axion(-like particles)}

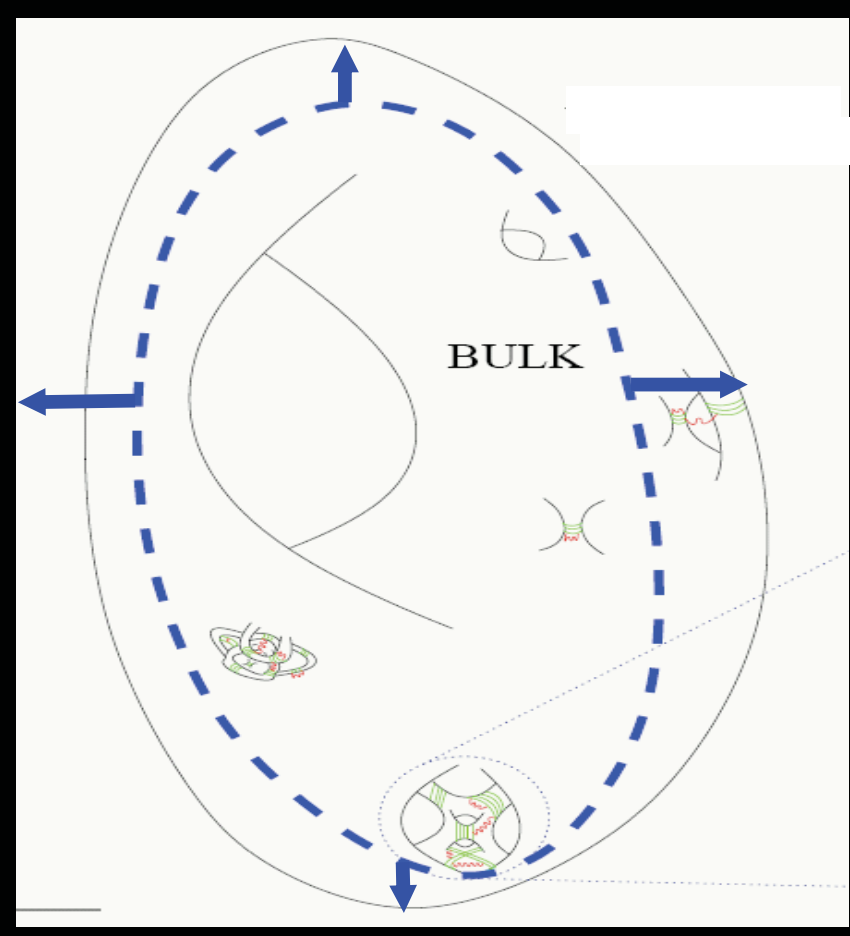


- Example: Axions

$$
\begin{gathered}
\mathcal{L}_{a}=\partial_{\mu} a \partial^{\mu} a-m_{a}^{2} a^{2}-\frac{1}{4} g a F^{\mu \nu} \tilde{F}_{\mu \nu}+\ldots \\
\text { Two-photon interaction! }
\end{gathered}
$$

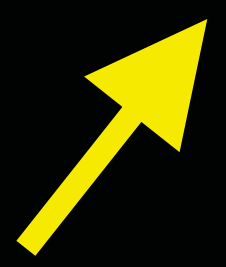

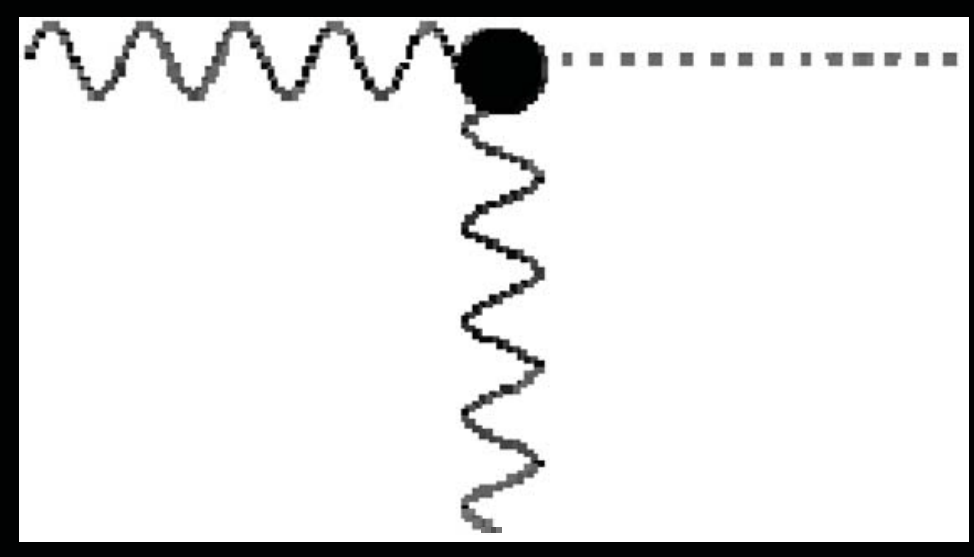




\section{Axion interactions}

- Light scalars or pseudoscalars

Can also have Yukawa couplings

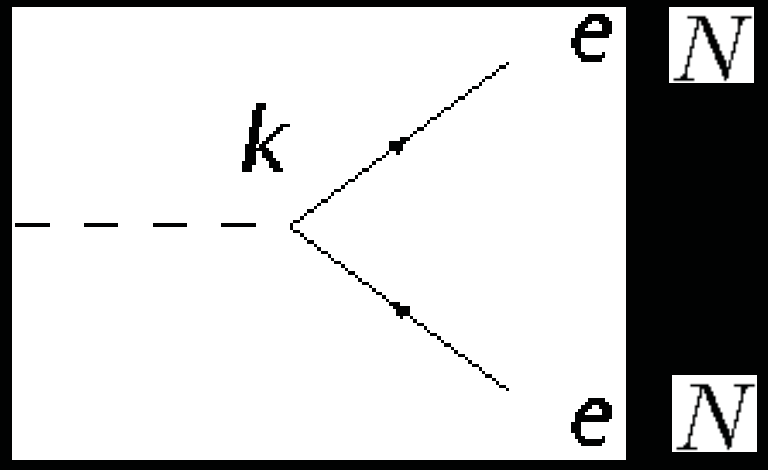

$k \sim$ dimensionless $\ll 1$

- Electrons, e

- Nucleons, N 


\section{Axion(-like particle) interactions}

- Light scalars or pseudoscalars

Can also have Yukawa couplings

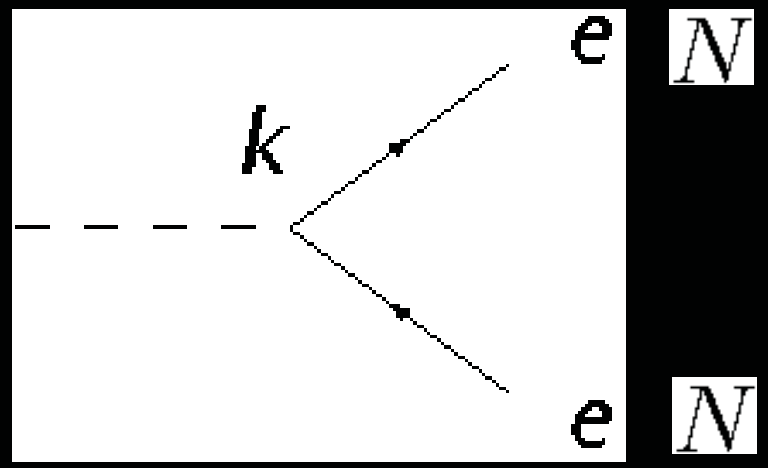

$k \sim$ dimensionless $\ll 1$

- Electrons, e

- Nucleons, N

For QCD axion:

$$
k_{e, N} \sim \frac{m_{e, N}}{f_{a}} \ll 1
$$




\section{Fidesn Photons}

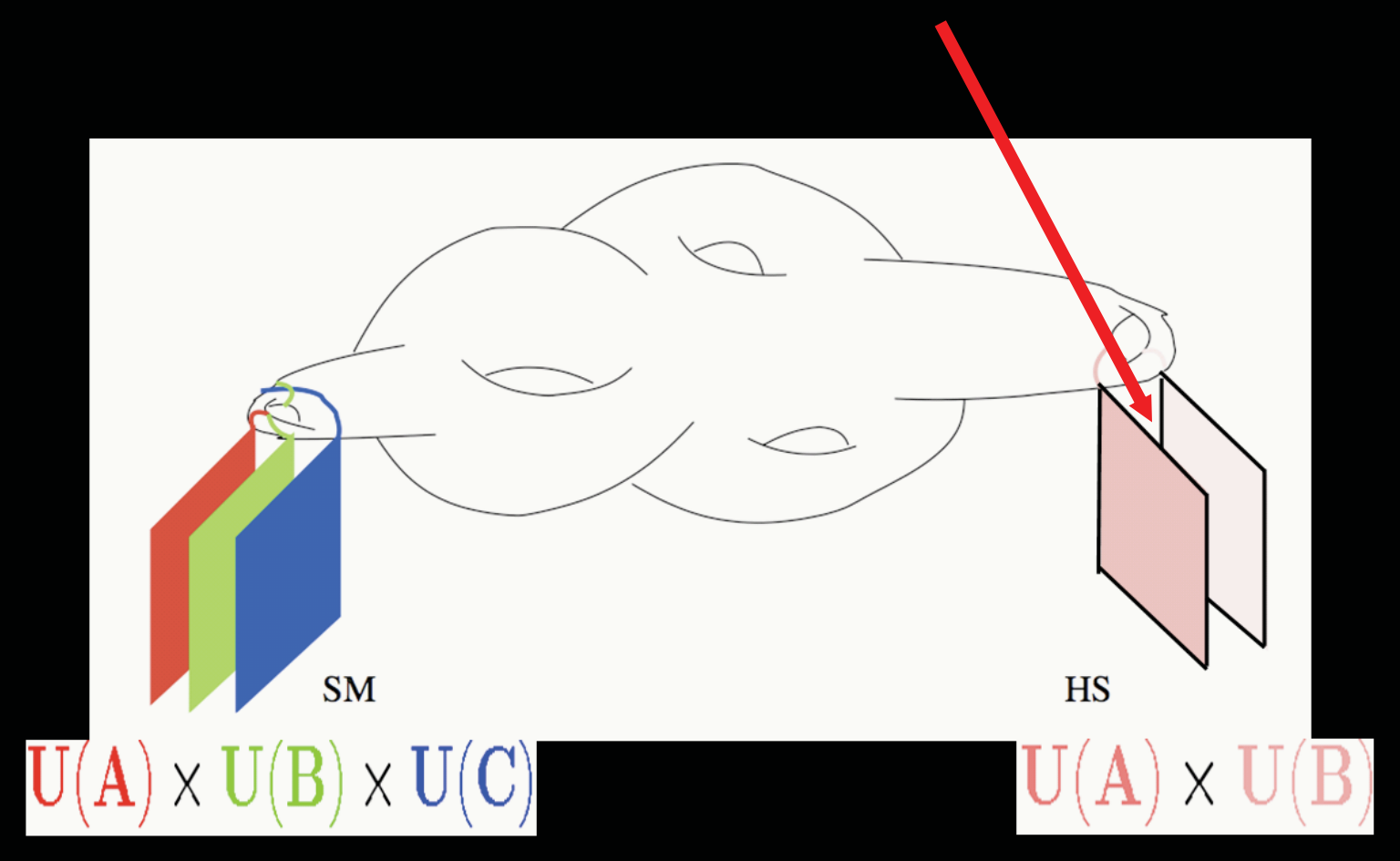


Hidden Photon interactions

- Kinetic mixing

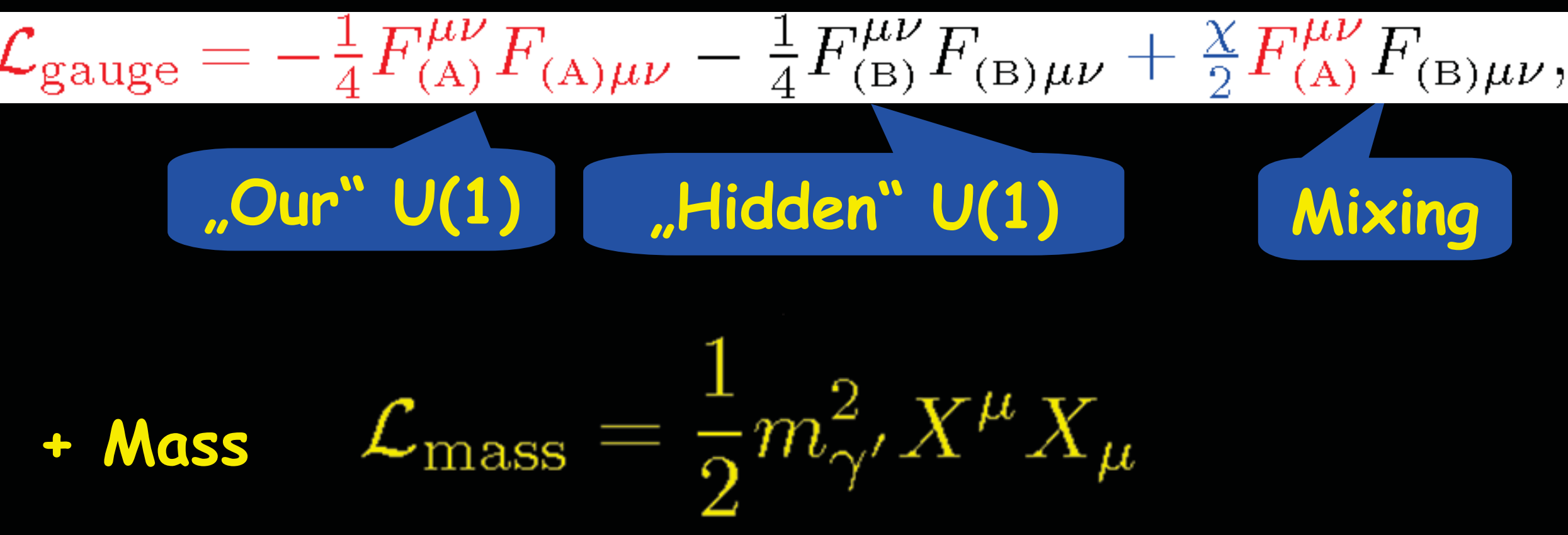




\section{Hidden Photons}

- Extra U(1) gauge boson, i.e. extra photon

- Normal matter not charged under it

- Main interaction:

Kinetic mixing

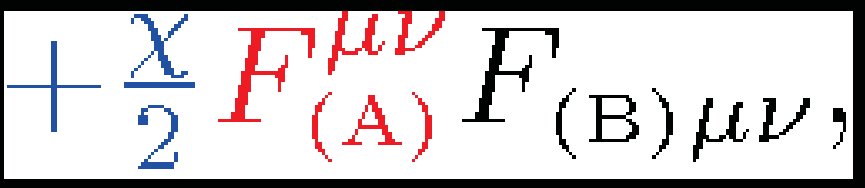

$\chi \sim$ dimensionless $\ll 1$

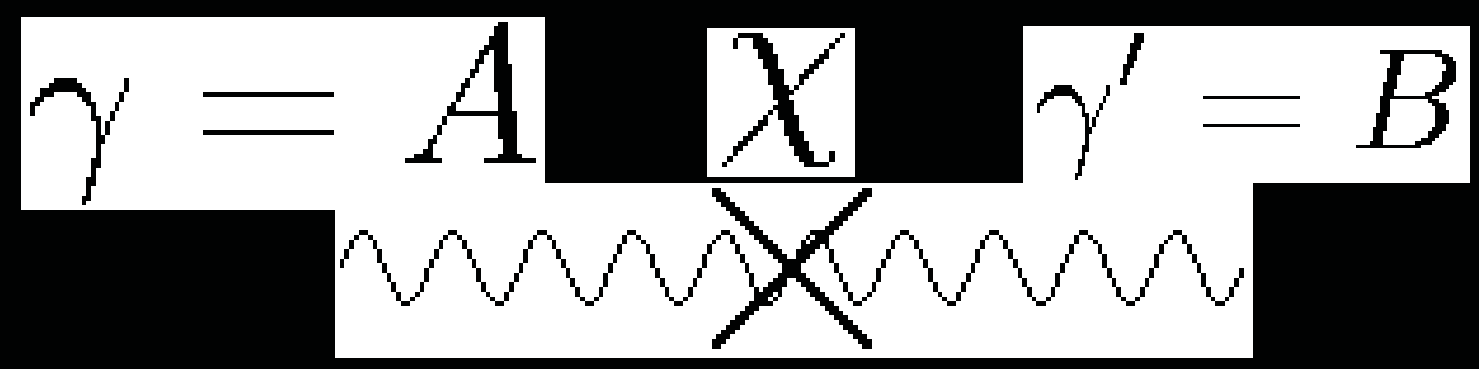


Hidden Photon: Different basis

- Kinetic mixing

$\mathcal{L}_{\text {gauge }}=-\frac{1}{4} F_{(\mathrm{A})}^{\mu \nu} F_{(\mathrm{A}) \mu \nu}-\frac{1}{4} F_{(\mathrm{B})}^{\mu \nu} F_{(\mathrm{B}) \mu \nu}+\frac{\chi}{2} F_{(\mathrm{A})}^{\mu \nu} F_{(\mathrm{B}) \mu \nu}$

+ Mass

$\mathcal{L}_{\text {mass }}=\frac{1}{2} m_{\gamma^{\prime}}^{2} X^{\mu} X_{\mu}$

+ Interactions with electrons

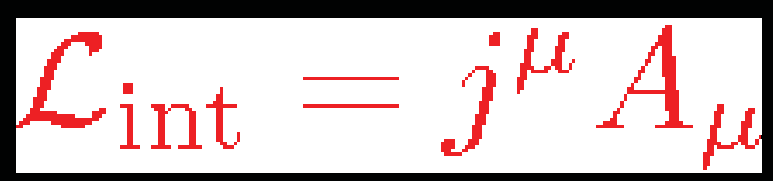




\section{Removing kinetic mixing}

$\mathcal{L}_{\text {gauge }}=-\frac{1}{4} F_{(\mathrm{A})}^{\mu \nu} F_{(\mathrm{A}) \mu \nu}-\frac{1}{4} F_{(\mathrm{B})}^{\mu \nu} F_{(\mathrm{B}) \mu \nu}+\frac{\chi}{2} F_{(\mathrm{A})}^{\mu \nu} F_{(\mathrm{B}) \mu \nu}$,

$+\mathcal{L}_{\text {mass }}=\frac{1}{2} m_{\gamma^{\prime}}^{2} B^{\mu} B_{\mu}$

9?

$$
\begin{aligned}
& =" A^{2}+B^{2}-2 \chi A B \\
& =A^{2}+(B+\chi A)^{2}+\mathcal{O}\left(\epsilon^{2}\right) \\
& =(A+\chi B)^{2}+B^{2}+\mathcal{O}\left(\epsilon^{2}\right)
\end{aligned}
$$

Diagonalization: $A^{\mu} \rightarrow \hat{A}^{\mu}-\chi B^{\mu}$ 


\section{Hidden Photon: Different basis}

- No Kinetic mixing for $\hat{A}$

$\mathcal{L}_{\text {gauge }}=-\frac{1}{4} \hat{F}_{(\mathrm{A})}^{\mu \nu} \hat{F}_{(\mathrm{A}) \mu \nu}-\frac{1}{4} F_{(\mathrm{B})}^{\mu \nu} F_{(\mathrm{B}) \mu \nu}$

+ Mass

$\mathcal{L}_{\text {mass }}=\frac{1}{2} m_{\gamma^{\prime}}^{2} B^{\mu} B_{\mu}$

+ Interactions with electrons (and other charges)

$\mathcal{L}_{\text {int }}=j^{\mu}\left(\hat{A}_{\mu}-\chi B_{\mu}\right)$

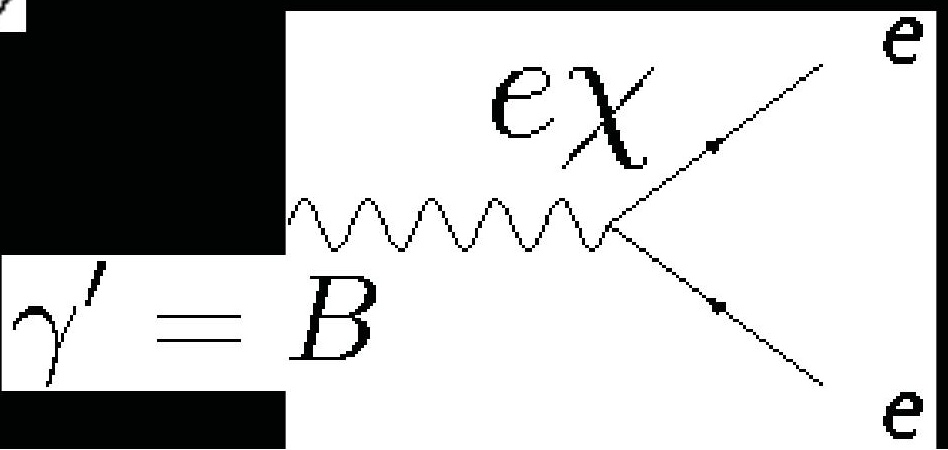




\section{Minicharged Particles}

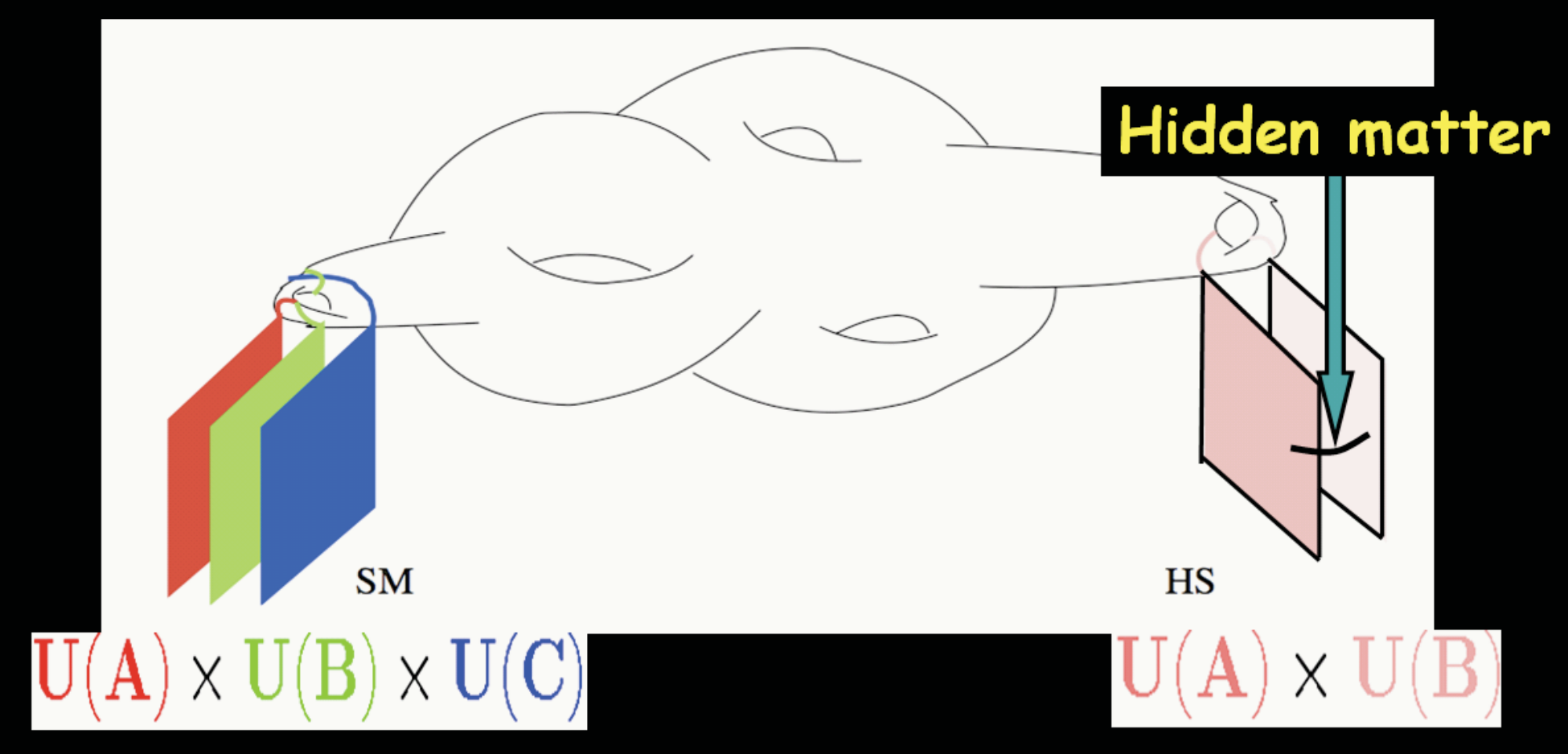




\section{How coupled?}

- Kinetic mixing

$\mathcal{L}_{\text {gauge }}=-\frac{1}{4} F_{(\mathrm{A})}^{\mu \nu} F_{(\mathrm{A}) \mu \nu}-\frac{1}{4} F_{(\mathrm{B})}^{\mu \nu} F_{(\mathrm{B}) \mu \nu}+\frac{\chi}{2} F_{(\mathrm{A})}^{\mu \nu} F_{(\mathrm{B}) \mu \nu}$,

\section{„Our" U(1) „Hidden" U(1)}

Mixing

+ Matter $\quad \mathcal{L}_{\text {int }}=g_{\text {hid }} \bar{h} \gamma_{\mu} X^{\mu} h$

Particles with small electric charges

LSW + other experiments 


\section{Kinetic Mixing - How to get Minicharges}

- Two U(1)'s

$\mathcal{L}_{\text {gauge }}=-\frac{1}{4} F_{(\mathrm{A})}^{\mu \nu} F_{(\mathrm{A}) \mu \nu}-\frac{1}{4} F_{(\mathrm{B})}^{\mu \nu} F_{(\mathrm{B}) \mu \nu}+\frac{\chi}{2} F_{(\mathrm{A})}^{\mu \nu} F_{(\mathrm{B}) \mu \nu}$,

\section{"Our" U(1) "Hidden" U(1)}

\section{Mixing}

$\begin{aligned} & =" A^{2}+B^{2}-2 \chi A B \\ & =A^{2}+(B+\chi A)^{2}+\mathcal{O}\left(\chi^{2}\right)\end{aligned}$

Diagonalization: $B^{\mu} \rightarrow B^{\mu}+\chi A^{\mu}$ 


\section{Kinetic Mixing - How to get Minicharges}

- Two U(1)'s

$\mathcal{L}_{\text {gauge }}=-\frac{1}{4} F_{(\mathrm{A})}^{\mu \nu} F_{(\mathrm{A}) \mu \nu}-\frac{1}{4} F_{(\mathrm{B})}^{\mu \nu} F_{(\mathrm{B}) \mu \nu}+\frac{\chi}{2} F_{(\mathrm{A})}^{\mu \nu} F_{(\mathrm{B}) \mu \nu}$,

\section{"Our" U(1) "Hidden" U(1)}

Mixing

Diagonalization: $B^{\mu} \rightarrow B^{\mu}+\chi A^{\mu}$

$g_{h} \overline{\mathbf{h}} B^{\mu} \mathbf{h} \rightarrow g_{h} \overline{\mathbf{h}} B^{\mu} \mathbf{h}+\chi g_{h} \overline{\mathbf{h}} A^{\mu} \mathbf{h}$ 


\section{Minicharged particles}

- Extra U(1) gauge boson, i.e. extra photon

- Normal matter not charged under it

- Main interaction:

- Small coupling to photons, related to kinetic mixing

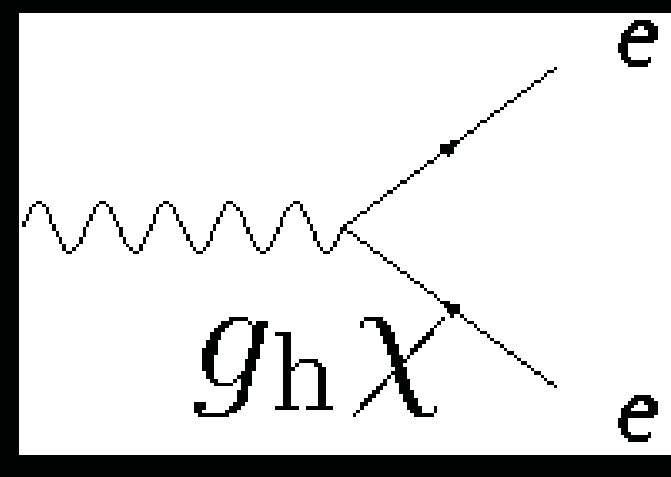

$\chi \sim$ dimensionless $\ll 1$

$g_{\mathrm{h}} \sim$ dimensionless $\sim 1$ or $\ll 1$ 


\section{Energy loss in}

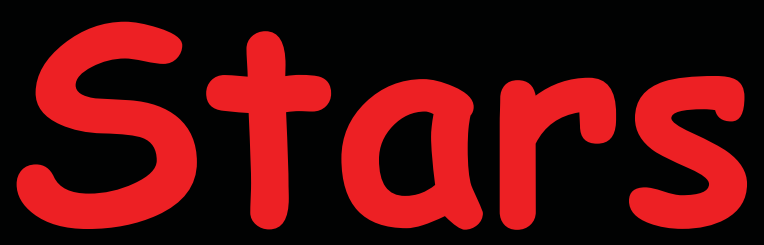

See

G. Raffelt

"Stars as Laboratories

for Fundamental Physics" 
Axions

- Primakoff proces

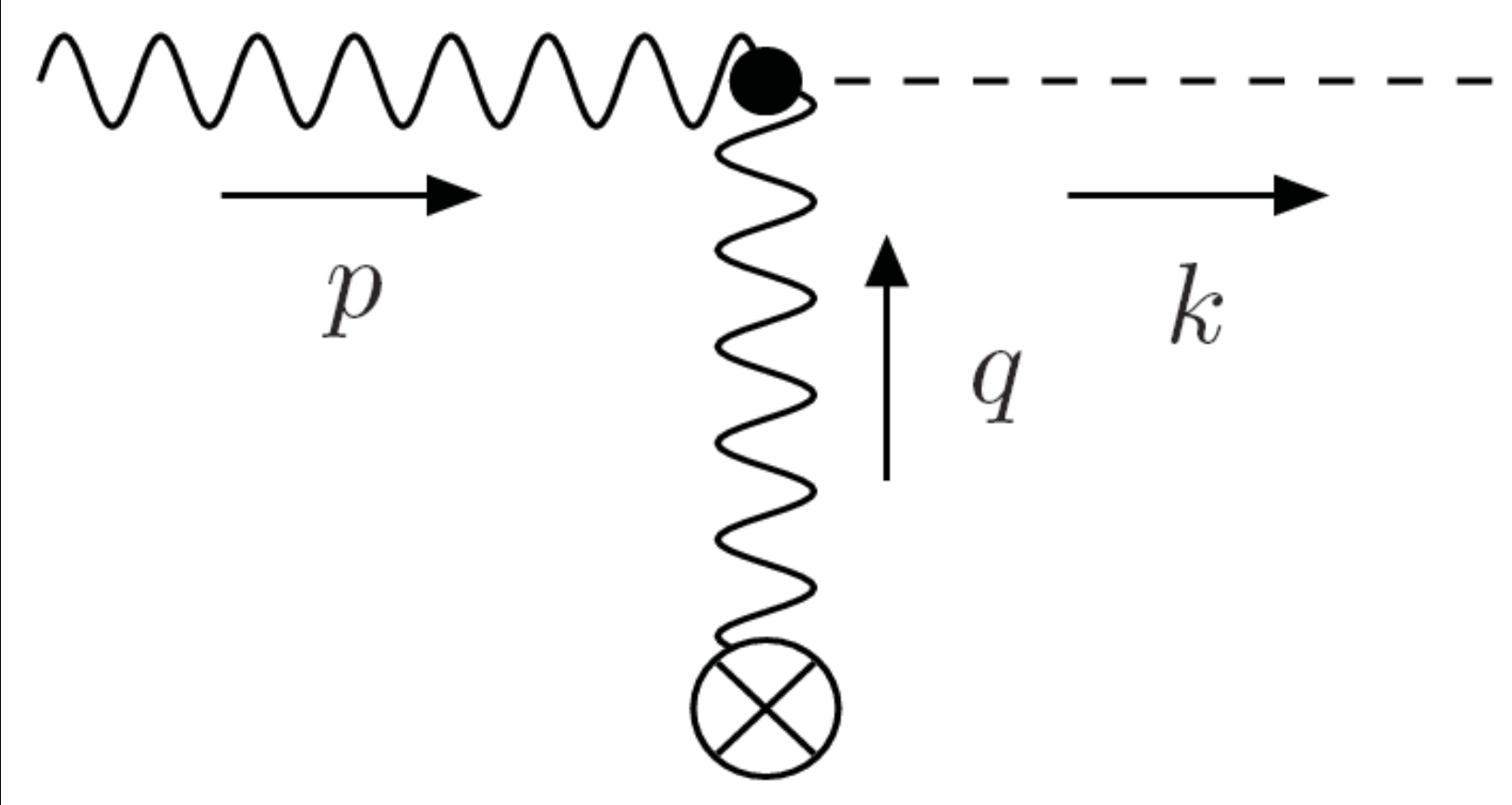


- Primakoff process (in the sun)

\section{ALP (leaving the sun)}

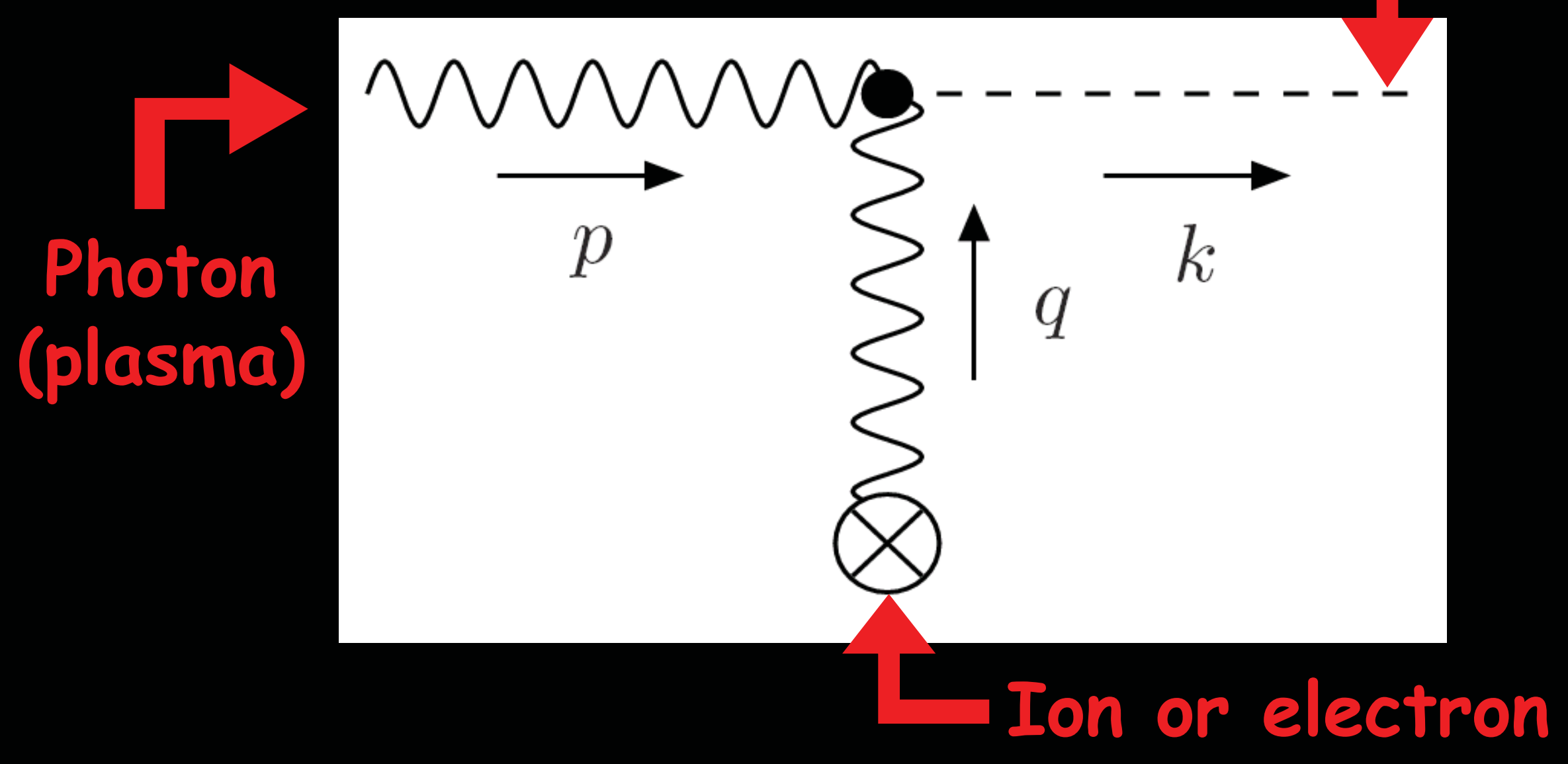


- If the coupling $g$ is too large the sun would have died long ago.

- Why?

Axions can leave the sun without further
interaction (in contrast to photons)

Large energy loss from axion emission

Sun burns fuel faster

Sun would have died long ago 


\section{A (Very) Moderate Bound}

- Without ALPs sun has fuel for about $10^{10}$ years

- Energy loss via ALPs:

$$
L_{a} \approx 1.710^{9}\left(g 10^{4} \mathrm{GeV}\right)^{2} L_{\gamma}
$$

- Sun Lifetime with ALPs

$$
t_{\text {sun }} \sim 10 \text { years } \times\left(g 10^{4} \mathrm{GeV}\right)^{-2}
$$

- Pretty sure sun has been around for more than 10 years

$$
g \leq \frac{1}{10^{4} \mathrm{GeV}}
$$




\section{Axion-like particles}

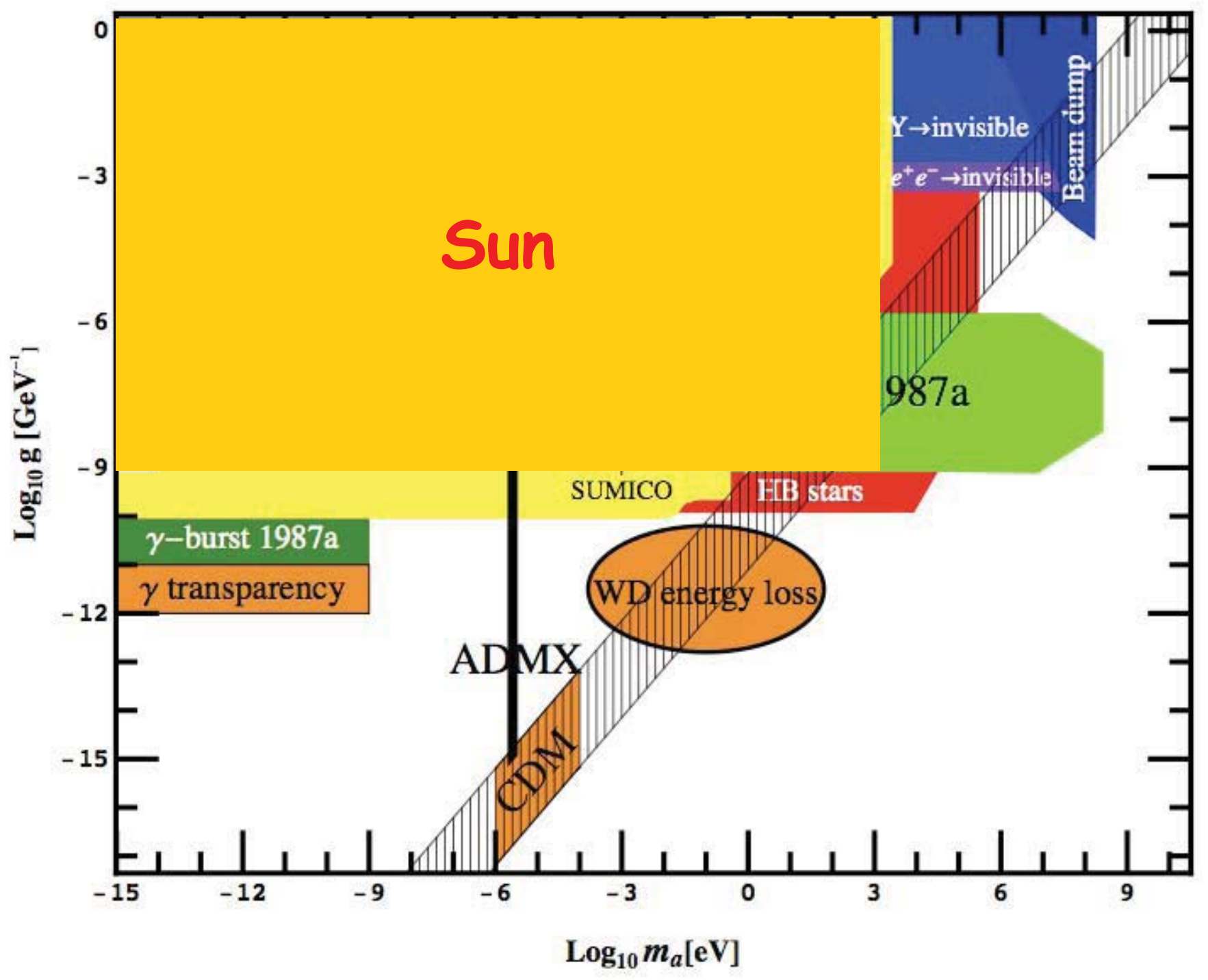




\section{Can do better...}

- Look at variety of stars

- Best are horizontal branch stars.

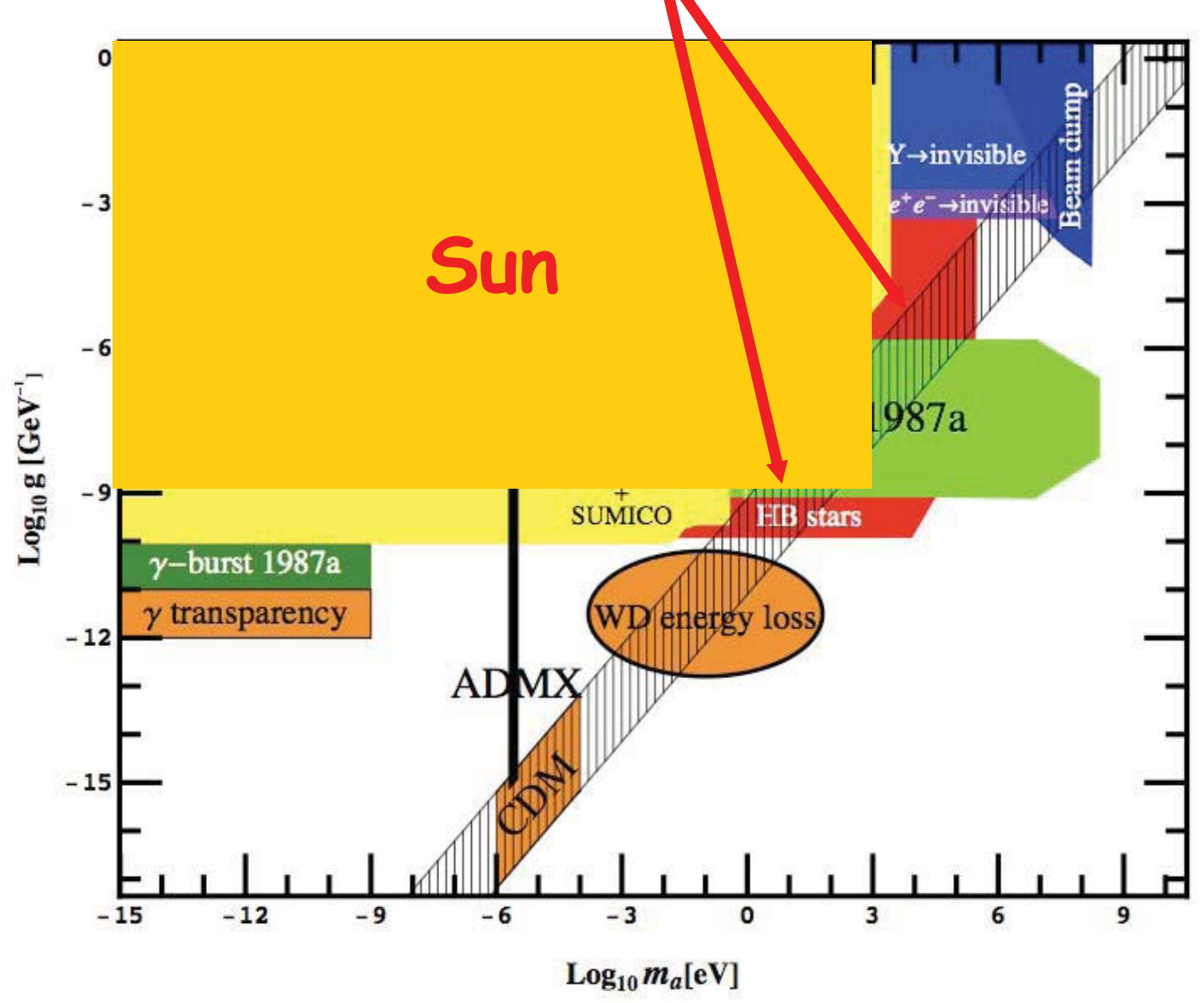




\section{Works also for hidden photons...}

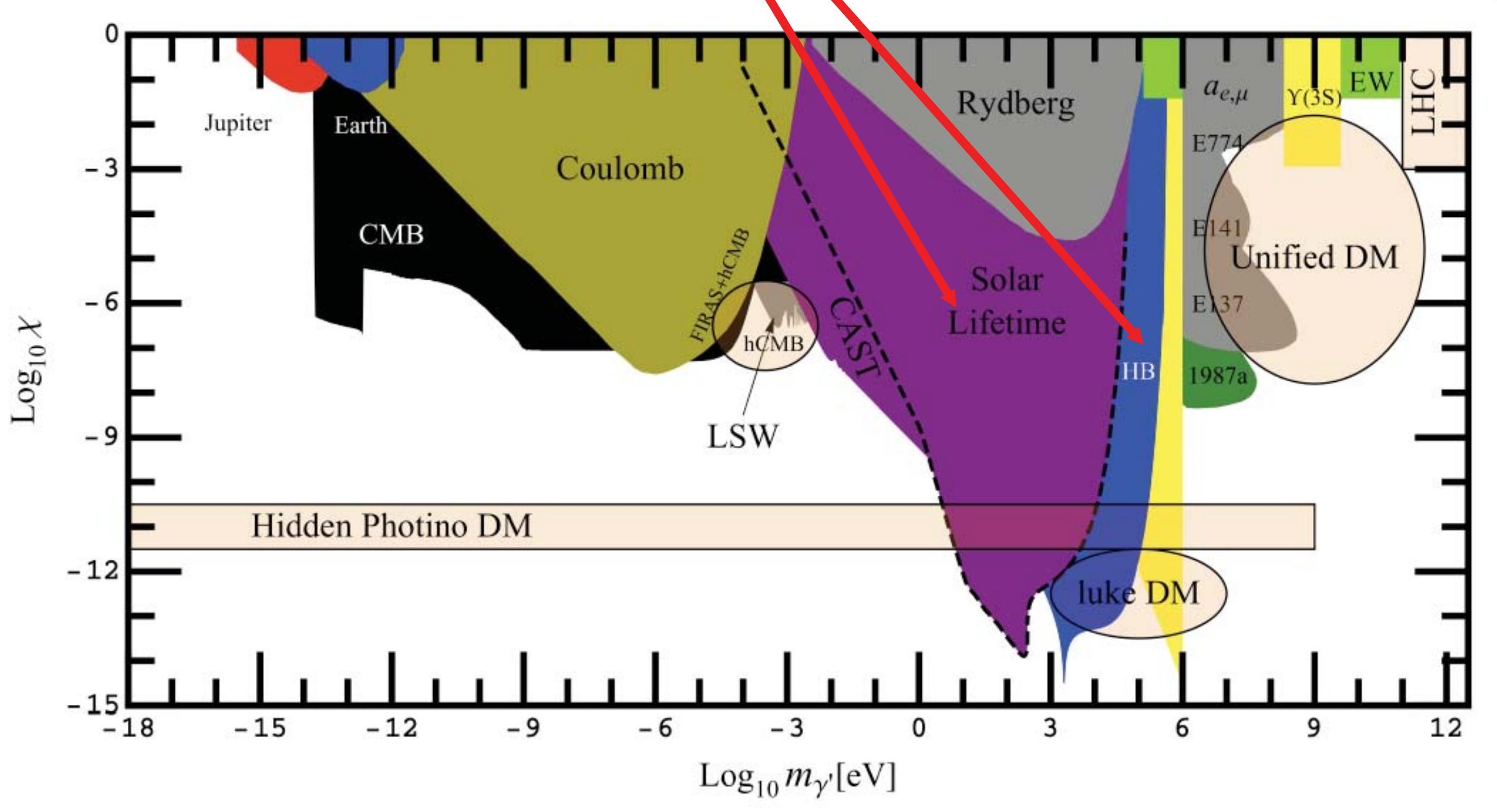




\section{...and minicharged particles}

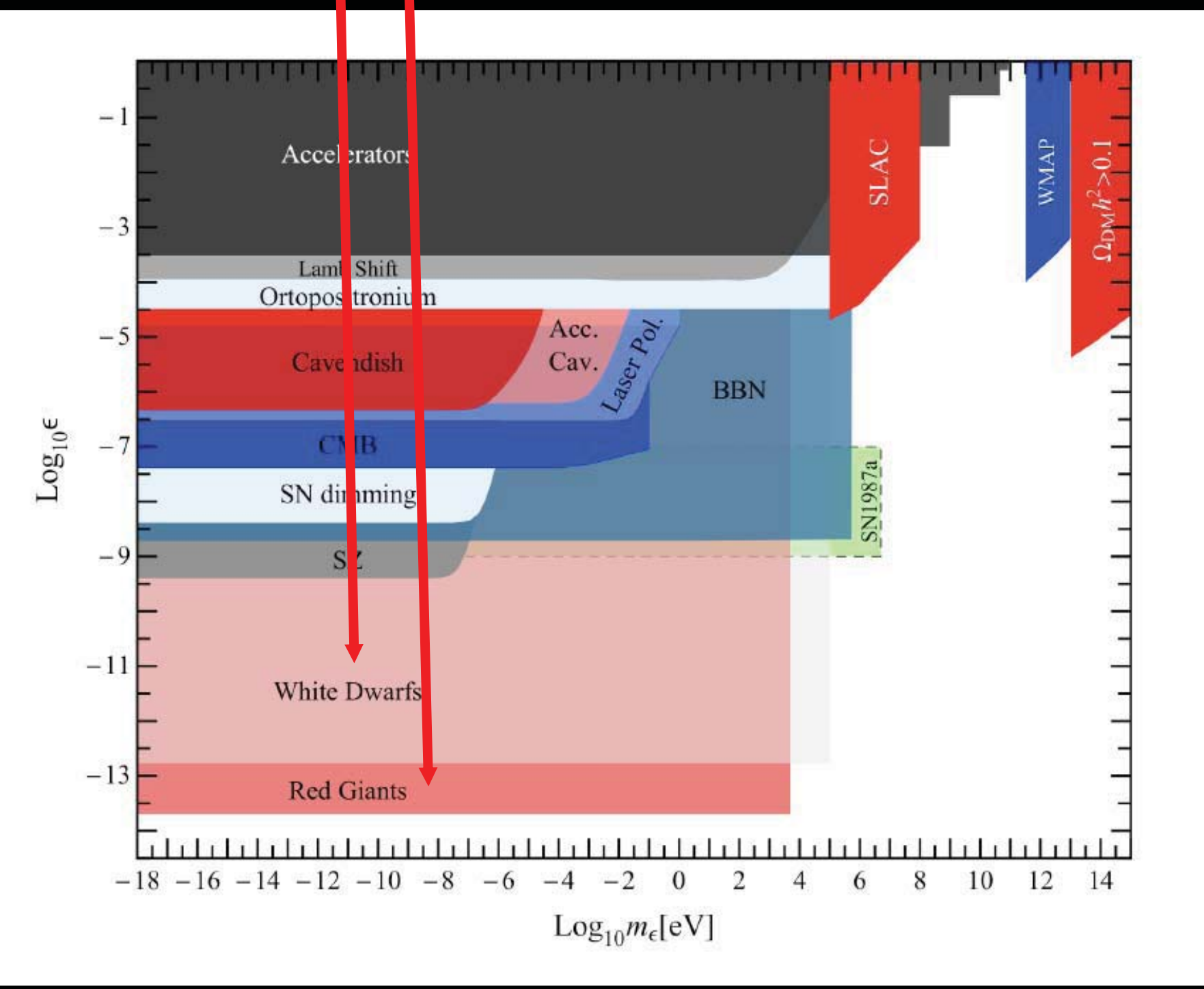


Not only bounds but also a new hint!!!

- White Dwarfs seem to loose a bit more energy than expected.

- This could be explained by an Axion(-like particle) coupled to electrons

- The corresponding two-photon coupling

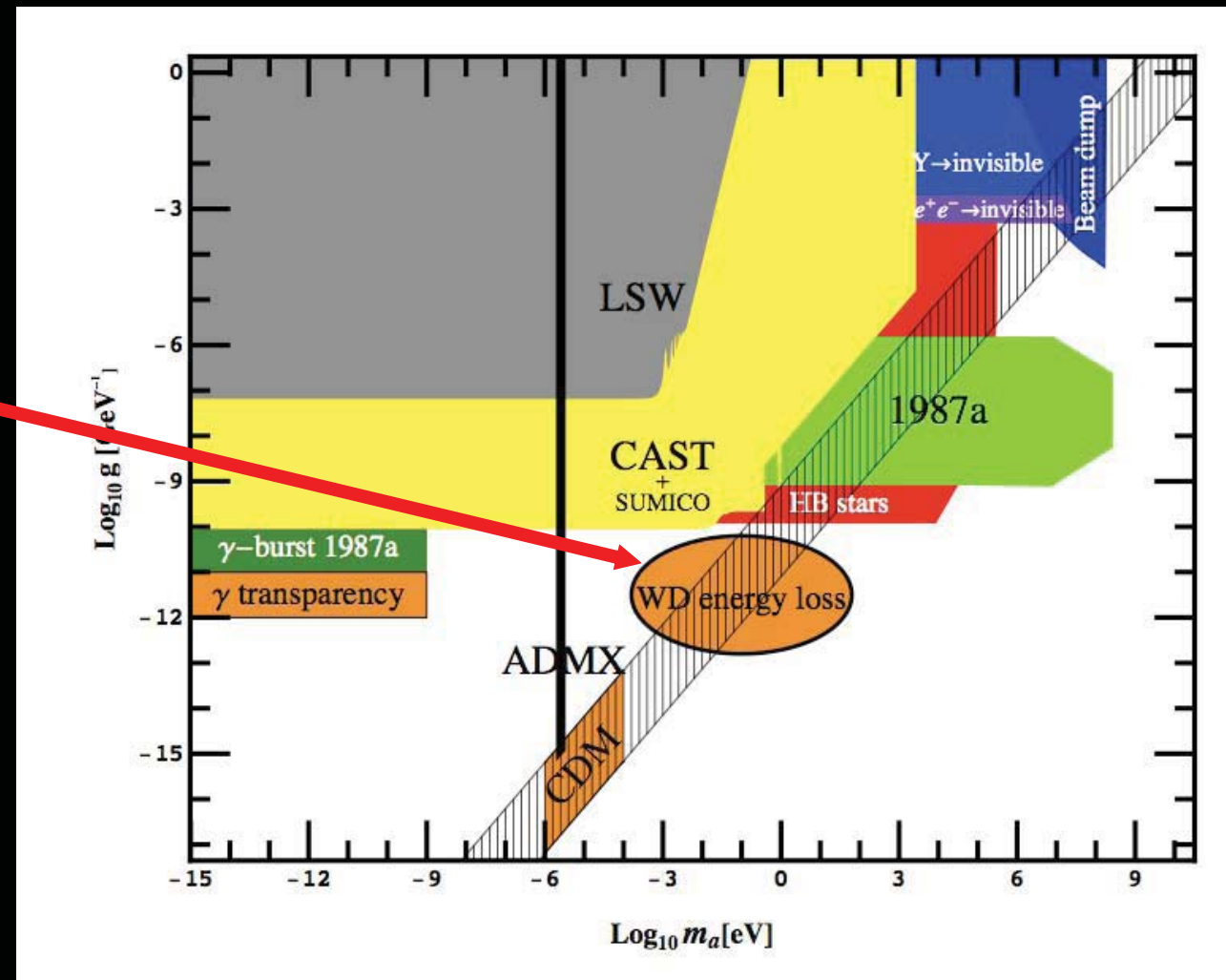




\section{Extra degrees of freedom at}

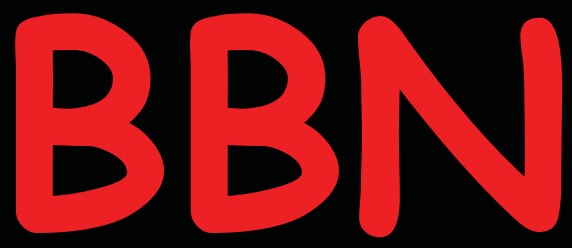

See, e.g.

E. Kolb and M. Turner

"The Early Universe"

Also S. Hannestad 2010 


\section{Basic facts of Big Bang Nucleosynthesis}

- After the quark-hadron transition

- Trfew $100 \mathrm{MeV}$, t 10-6 s

- Most hadrons are Pions. 


\section{Basic facts of Big Bang Nucleosynthesis}

- Later when $T \ll 100$ MeV $m_{\pi}$

- pions decay away

- Mostly neutrons and protons (+ electrons)

In equilibrium:

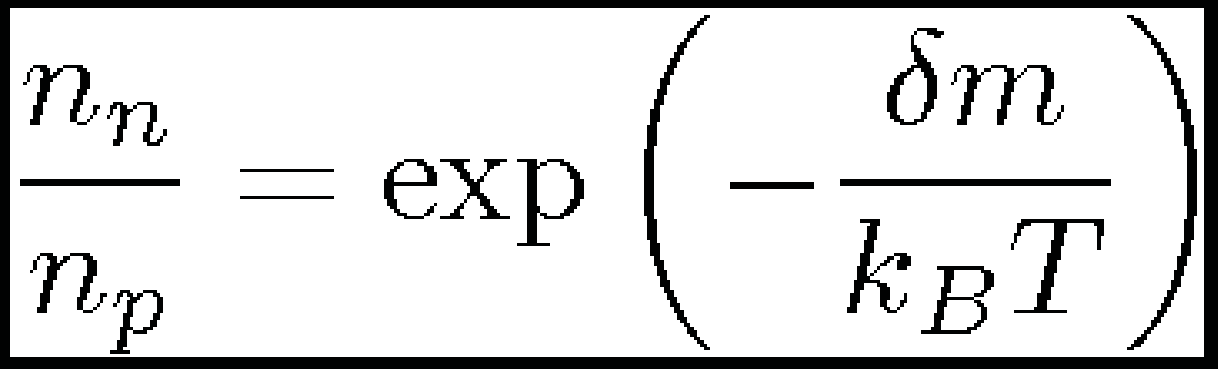

$\delta m=m_{n}-m_{p}=1.293 \mathrm{MeV}$ 


\section{Are we in eqilibrium?}

- $\boldsymbol{n - p}$ changing interactions $v_{e}+n \leftrightarrow e^{-}+p$

$$
\begin{aligned}
& e^{+}+n \leftrightarrow \bar{v}_{e}+p \\
& n \leftrightarrow e^{-}+p+\bar{v}_{e}
\end{aligned}
$$

- Rate

$$
\Gamma_{n-p}=n\langle\sigma v\rangle \sim T^{3} G_{F}^{2} T^{2}
$$

- Hubble

$$
H=\sqrt{\frac{8 \pi}{3} \frac{\rho}{m_{P l}^{2}}} \sim 1.66 \sqrt{g_{\star}} \frac{T^{2}}{m_{P l}}
$$

- Freeze out:

$$
\Gamma_{n-p}<H \Rightarrow T_{\text {freeze }} \sim 1 \mathrm{MeV}
$$




\section{Are we in eqilibrium?}

- Freeze out:

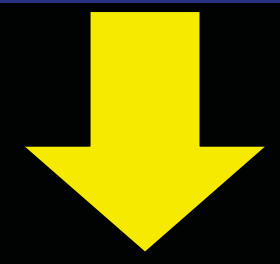

$\Gamma_{n-p}<H \Rightarrow T_{\text {freeze }} \sim 1 \mathrm{MeV}$

- At this point in time $\frac{n_{n}}{n_{p}} \sim \exp \left(-\frac{m_{n}-m_{p}}{T_{\text {freeze }}}\right) \frac{1}{6}$

- From then on: Neutrons decay with $\tau_{n}=886 \mathrm{~s}$ 
Nucleosynthesis...

- The first process is

$$
p+n \leftrightarrow D+\gamma
$$

- Naively it should start when

$$
T<m_{D}-m_{p}-m_{n} \sim 2.2 \mathrm{MeV}
$$

- However much much more $\gamma$ than $p$, n!l!

$$
\eta=\frac{n_{B}}{n_{\gamma}} \sim 10^{-10}
$$

Need: $\Gamma_{\text {production }}(D)=\Gamma_{\text {destruction }}(D)$ 
Nucleosynthesis...

$$
\left.\begin{array}{rl}
\Gamma_{\text {production }} & \approx n_{B}\langle\sigma v\rangle \\
\Gamma_{\text {destruction }} & \approx n_{\gamma}\langle\sigma v\rangle e^{-E_{b} / T}
\end{array}\right\} \Rightarrow T_{B B N} \approx-\frac{E_{b}}{\ln (\eta)} \approx 0.2 \mathrm{MeV}
$$

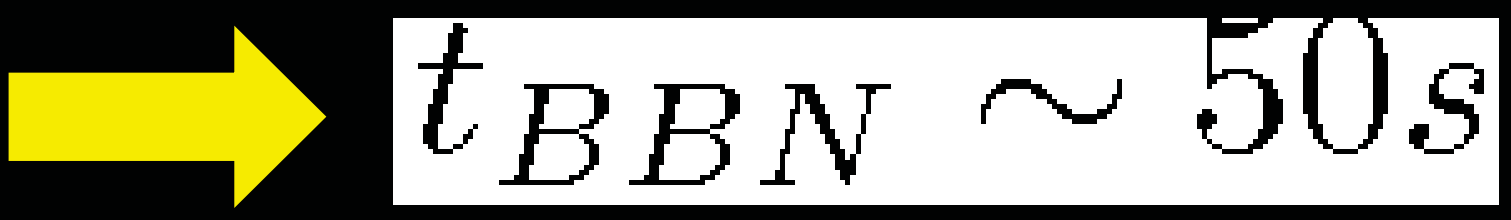

- At this point in time

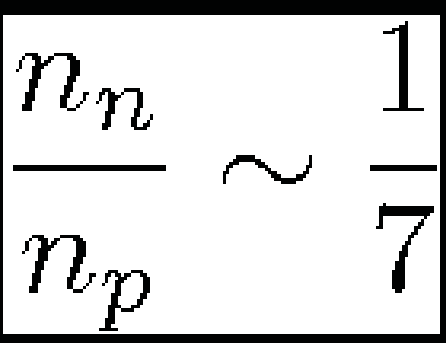


Nucleosynthesis...

- After formation of deuterium everything goes quickly

- Nearly all neutrons end in helium.

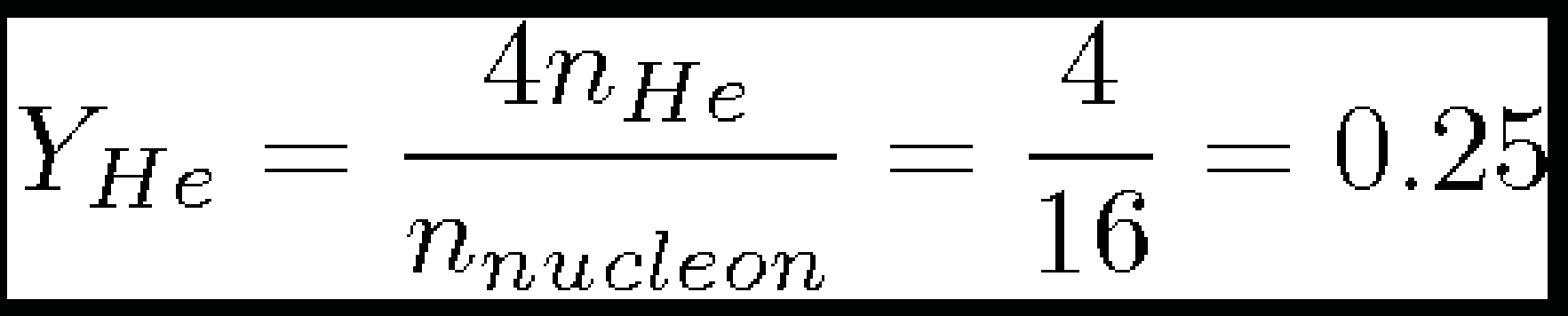

- This is roughly what is observed! 


\section{Extra species...}

- Extra light particles in equilibrium increase the Hubble constant

$\rightarrow$ (Smallish) changes in

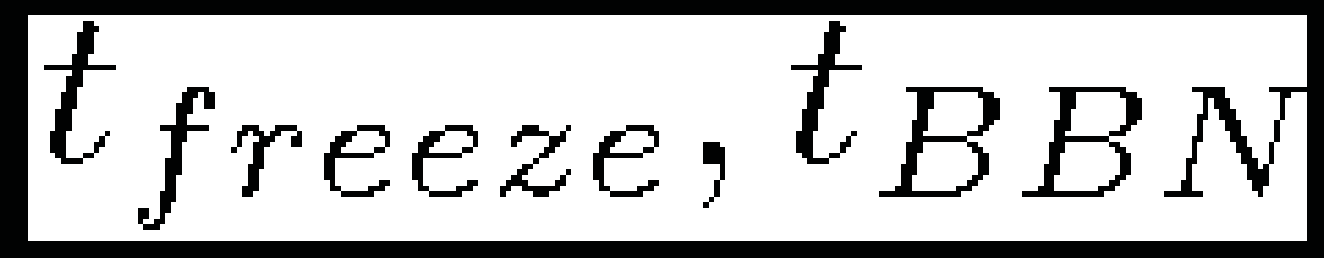

$\Rightarrow$ Changes $\mathrm{Y}_{\mathrm{He}}$ (and other element abundances) 


\section{Extra species...}

\section{WMAP}

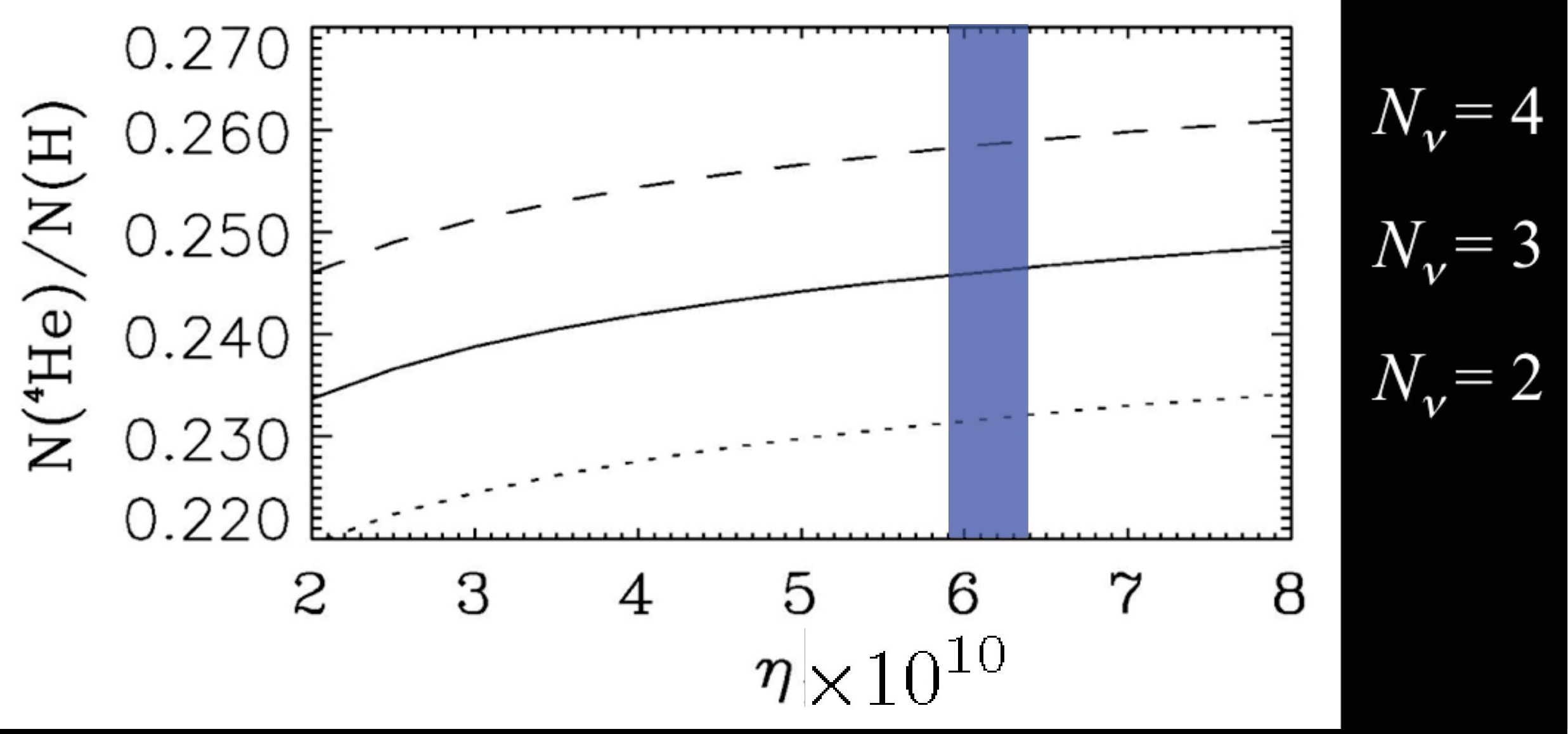




\section{Measured abundancies...}

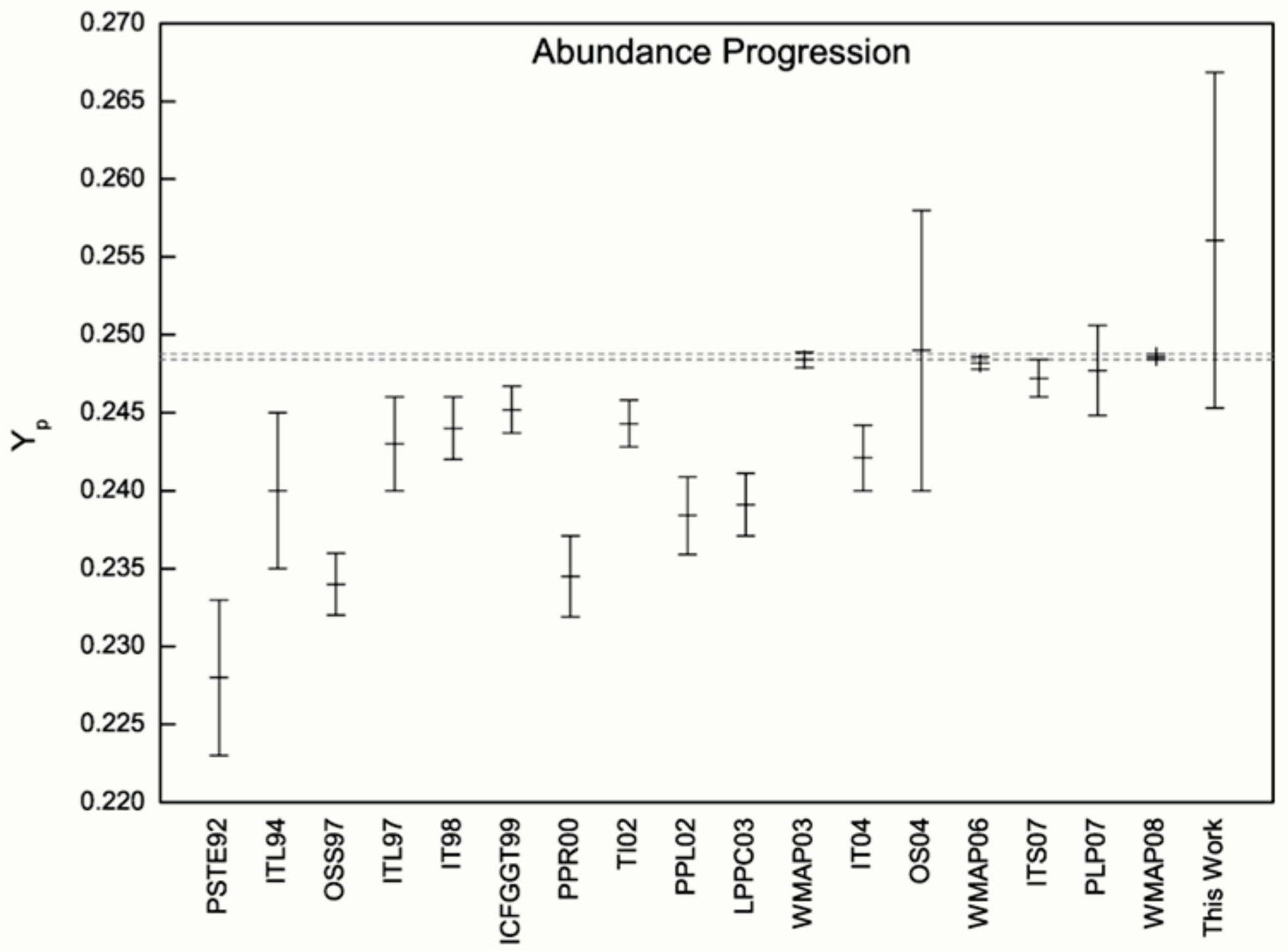




\section{Extra species...}

- Extra light particles in equilibrium increase the Hubble constant

$\rightarrow$ (Smallish) changes in

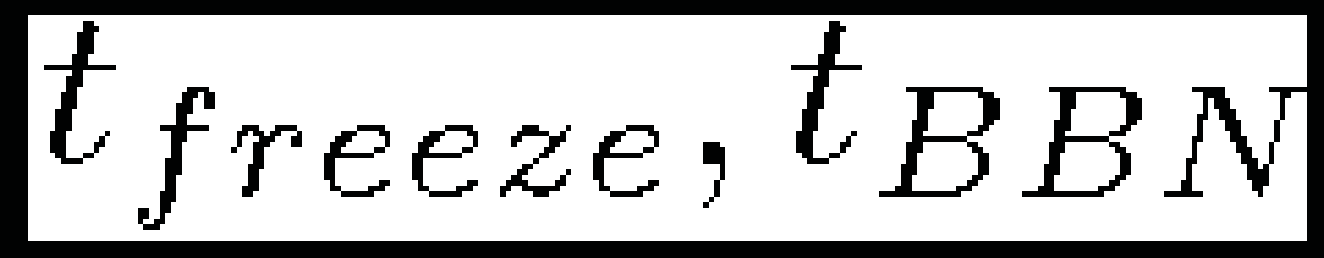

$\rightarrow$ Changes $Y_{\mathrm{He}}$ (and other element abundances)

We can constrain (or have a hint :-)):

$n$ light species

$\curvearrowright$ 


\section{Example of constraint: Minicharges}

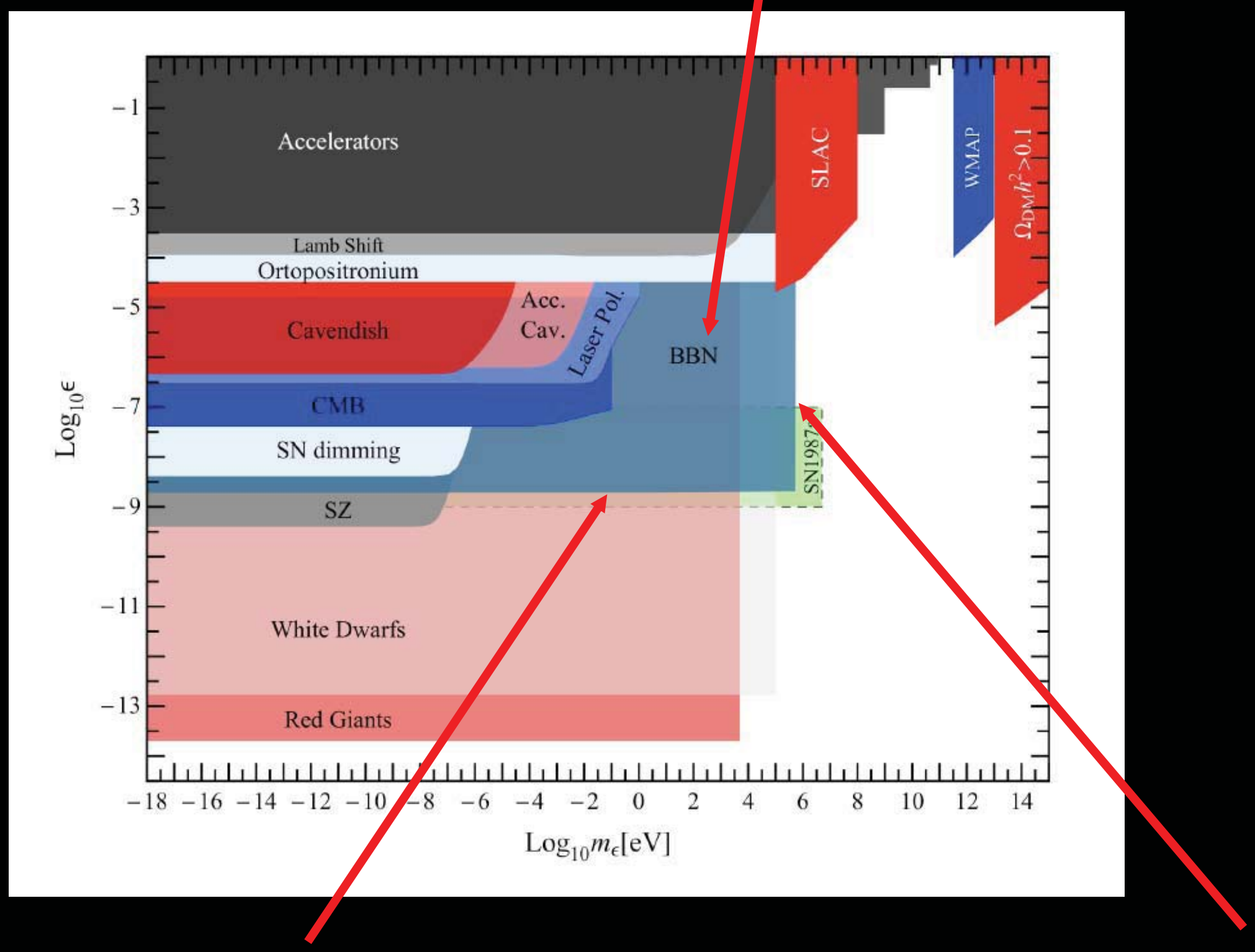

Not in equilibrium

Not enough energy 


\section{Axion-like particles}

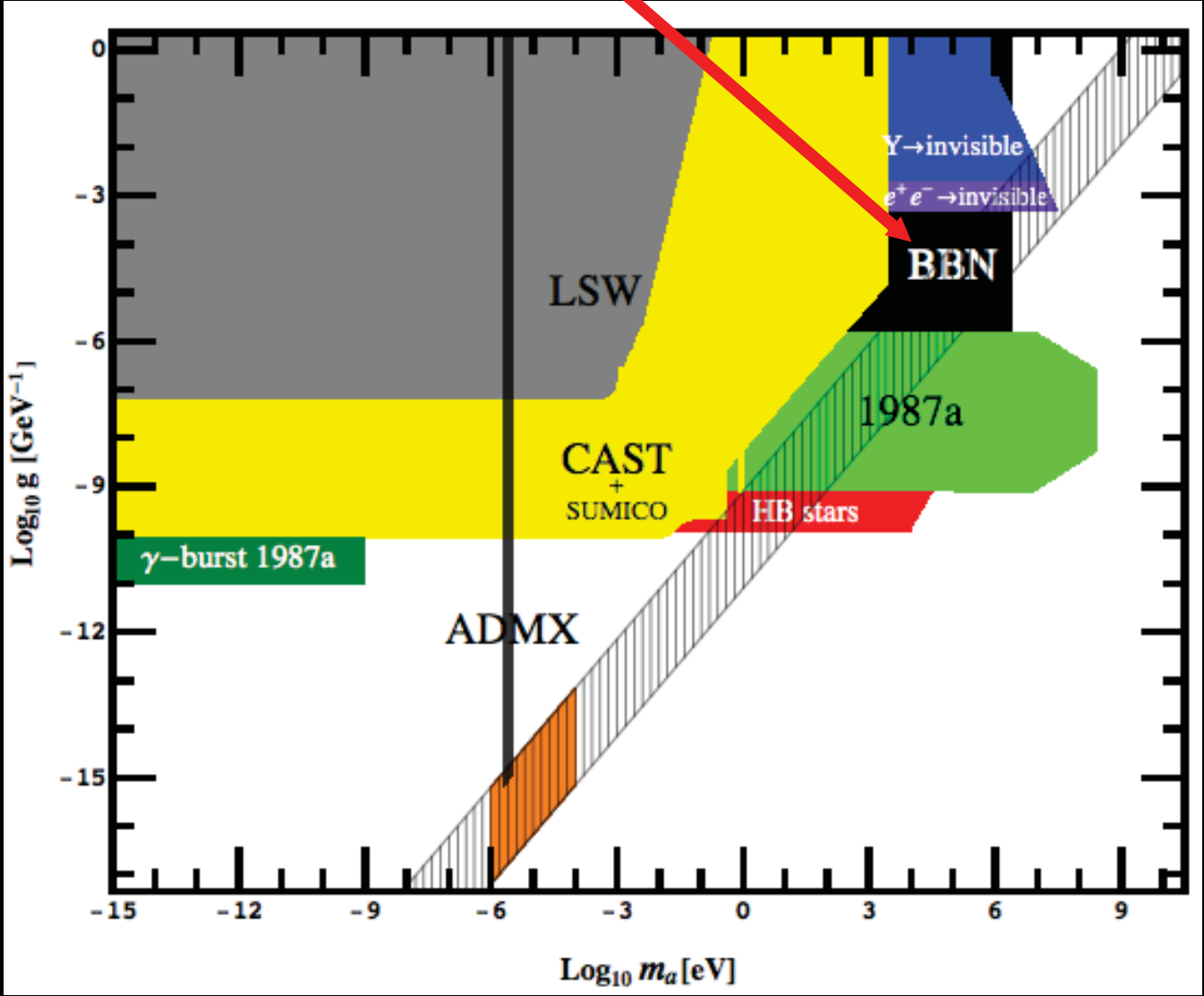

- Not my favourite bound: only 1 extra degree of freedom.

- Need to use one of the optimistic error estimate 


\section{Intermezzo}

Photon-WISP oscillations 
- In many situations we have coherent interactions over long distances.

- Multiple interactions...

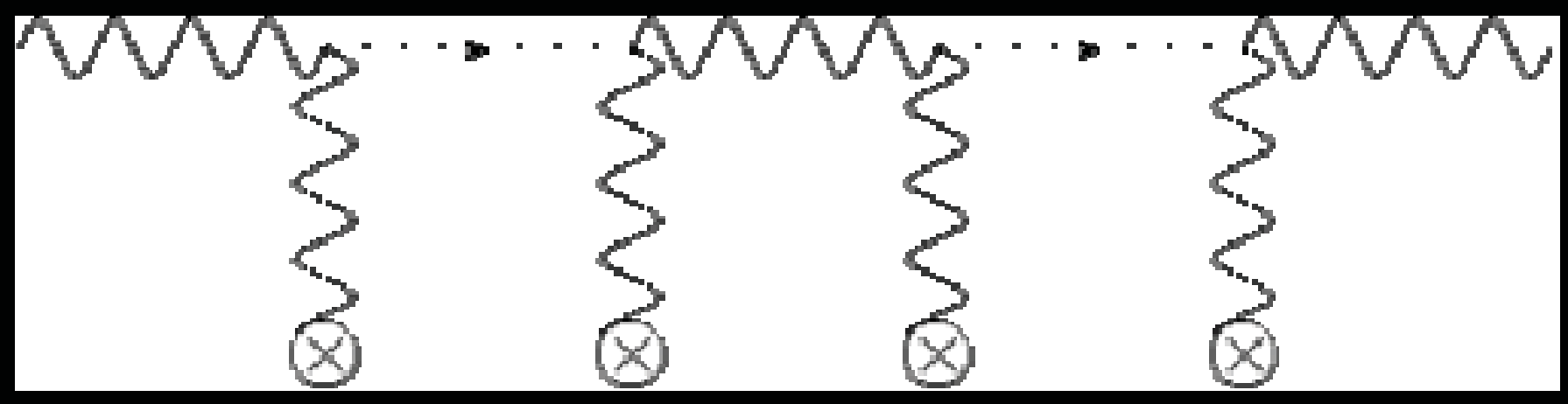

- All tree-level

Use classical equations of motion! 


$$
\left[\omega^{2} \mathbf{1}+\partial_{z}^{2} \mathbf{1}-\mathcal{M}^{X}\right]\left(\begin{array}{c}
A \\
X
\end{array}\right)=0
$$

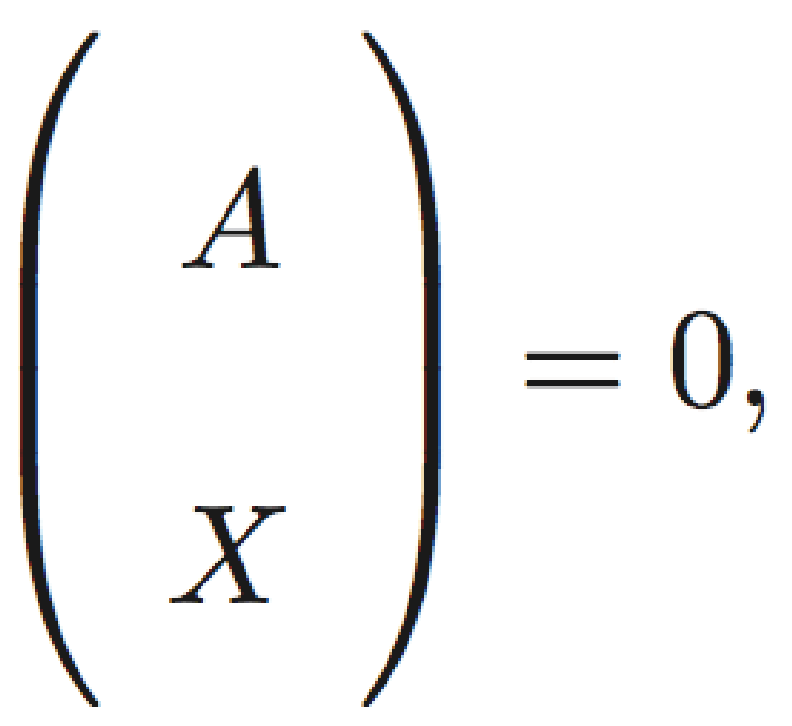

- Axion-like particles

$$
\mathcal{M}_{\|}^{a^{-}}=\left(\begin{array}{cc}
0 & -g B \omega \\
-g B \omega & m_{a}^{2}
\end{array}\right)
$$




$$
\left[\omega^{2} \mathbf{1}+\partial_{z}^{2} \mathbf{1}-\mathcal{M}^{X}\right]\left(\begin{array}{c}
A \\
X
\end{array}\right)=0
$$

- Hidden photons

$$
\mathcal{M}^{\gamma^{\prime}}=m_{\gamma^{\prime}}^{2}\left(\begin{array}{cc}
\chi^{2} & -\chi \\
-\chi & 1
\end{array}\right)
$$

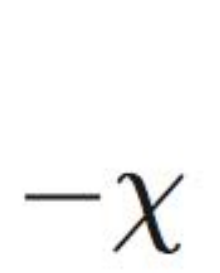


$v_{1}=\exp \left(-\mathbf{i}\left(\omega t-k_{1} z\right)\right)\left(\begin{array}{c}1 \\ \delta\end{array}\right), \quad v_{2}=\exp \left(-\mathbf{i}\left(\omega t-k_{2} z\right)\right)\left(\begin{array}{c}-\delta \\ 1\end{array}\right)$

- Propagation eigenstates

$\neq$ interaction eigenstates

- Photons mix with new particles!!

- Analog of neutrino oscillations! 


\section{Solutions... Details}

$v_{1}=\exp \left(-\mathbf{i}\left(\omega t-k_{1} z\right)\right)\left(\begin{array}{c}1 \\ \delta\end{array}\right), \quad v_{2}=\exp \left(-\mathbf{i}\left(\omega t-k_{2} z\right)\right)\left(\begin{array}{c}-\delta \\ 1\end{array}\right)$

In general:

$$
\tan (2 \delta)=2 \frac{\mathcal{M}_{12}^{X}}{\mathcal{M}_{11}^{X}-\mathcal{M}_{22}^{X}},
$$$$
k_{1}^{2}=\omega^{2}-\mathcal{M}_{11}^{X}, \quad k_{2}^{2}=\omega^{2}-\mathcal{M}_{22}^{X} .
$$ 


\section{Solutions... Details}

$v_{1}=\exp \left(-\mathbf{i}\left(\omega t-k_{1} z\right)\right)\left(\begin{array}{c}1 \\ \delta\end{array}\right), \quad v_{2}=\exp \left(-\mathbf{i}\left(\omega t-k_{2} z\right)\right)\left(\begin{array}{c}-\delta \\ 1\end{array}\right)$

For hidden photons:

$\delta \approx$

$\chi$

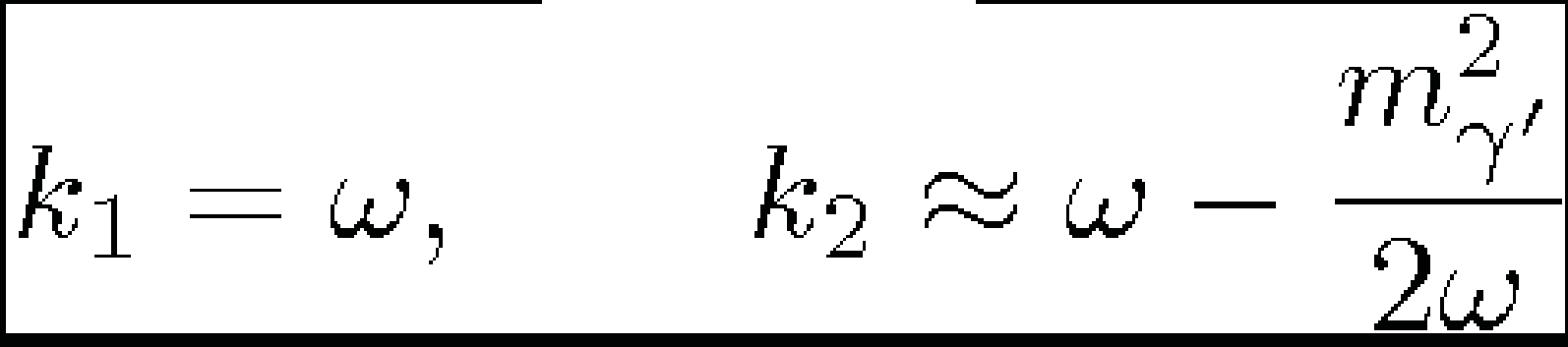




\section{And finally oscillations}

$A\left(\gamma \rightarrow \gamma^{\prime}\right)=\chi\left[\exp \left(i k_{1} x\right)-\exp \left(i k_{2} x\right)\right]$

$$
=\chi \exp (i \omega x)\left[1-\exp \left(\frac{m_{\gamma^{\prime}}^{2}}{2 \omega} x\right)\right.
$$

$P\left(\gamma \rightarrow \gamma^{\prime}\right)=4 \chi^{2} \sin ^{2}\left(\frac{m_{\gamma^{\prime}}^{2}}{4 \omega} x\right)$

Exactly the same structure as neutrino oscillations!!! 
Modifications of the CMB 
- We can have photon - hidden photon (WISP) oscillations!

- Small complication: Plasma $\Rightarrow$ photon plasma mass!

$$
\tan (2 \delta)=2 \frac{\mathcal{M}_{11}^{X}}{\mathcal{M}_{11}^{X}-\mathcal{M}_{22}^{X}},
$$


- We can have photon - hidden photon (WISP) oscillations!

- Small complication: Plasma $\Rightarrow$ photon plasma mass!

$$
\tan (2 \delta)=2 \frac{\mathcal{M}_{12}^{X}}{\mathcal{M}_{11}^{X}-\mathcal{M}_{22}^{X}}
$$
Hidden photon mass 
- We can have photon - hidden photon (WISP) oscillations!

- Small complication: Plasma $\Rightarrow$ photon plasma mass!

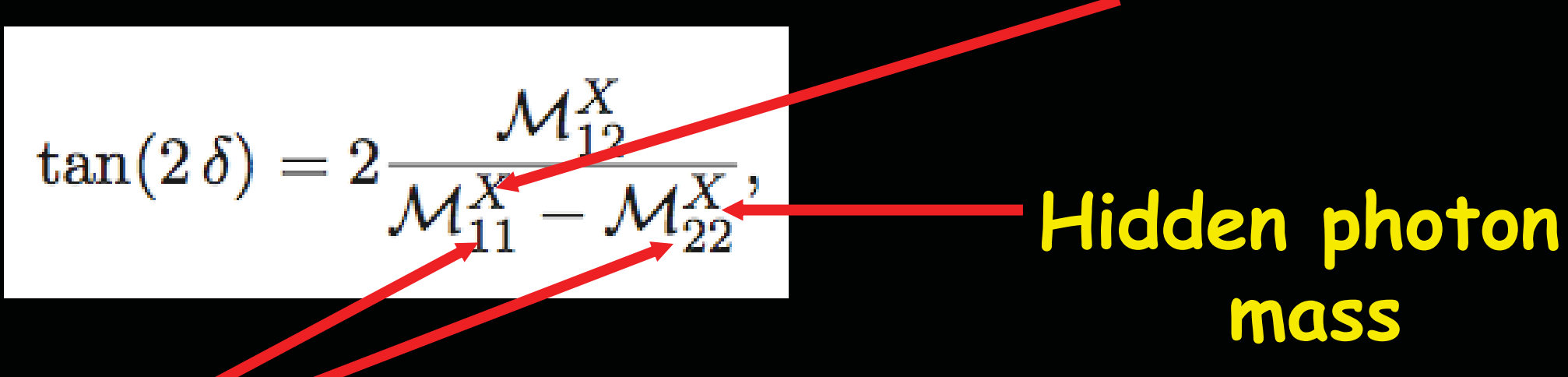

Can be equal $\Rightarrow$ Resonance!

$$
\text { Big effect! }
$$


- It transfers energy from Photons to hidden photons
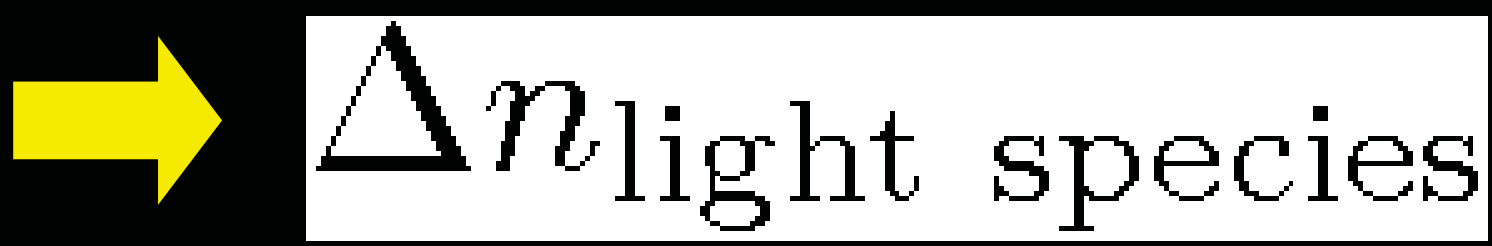

- It is energy dependent! $P\left(\gamma \rightarrow \gamma^{\prime}\right)=4 \chi^{2} \sin ^{2}\left(\frac{m_{\gamma^{\prime}}^{2}}{4 \omega}\right)$

Can distort the CMB blackbody spectrum!l! 


\section{Powerful bounds...}

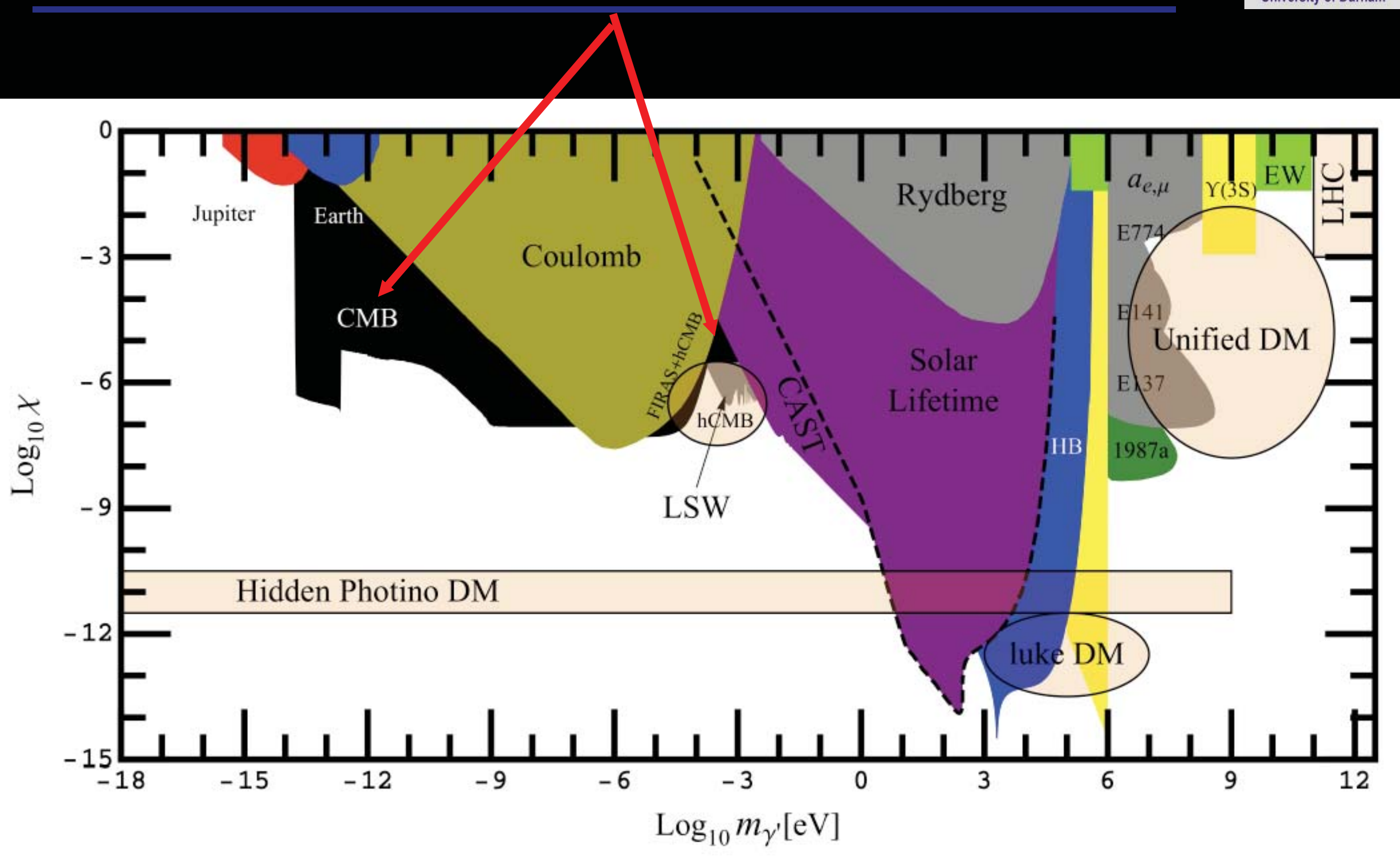

JJ, J. Redondo and A. Ringwald 0804.4157 A. Mirizzi, J. Redondo and G. Sigl 0901.0014 


\section{Powerful bounds... and a hint!}

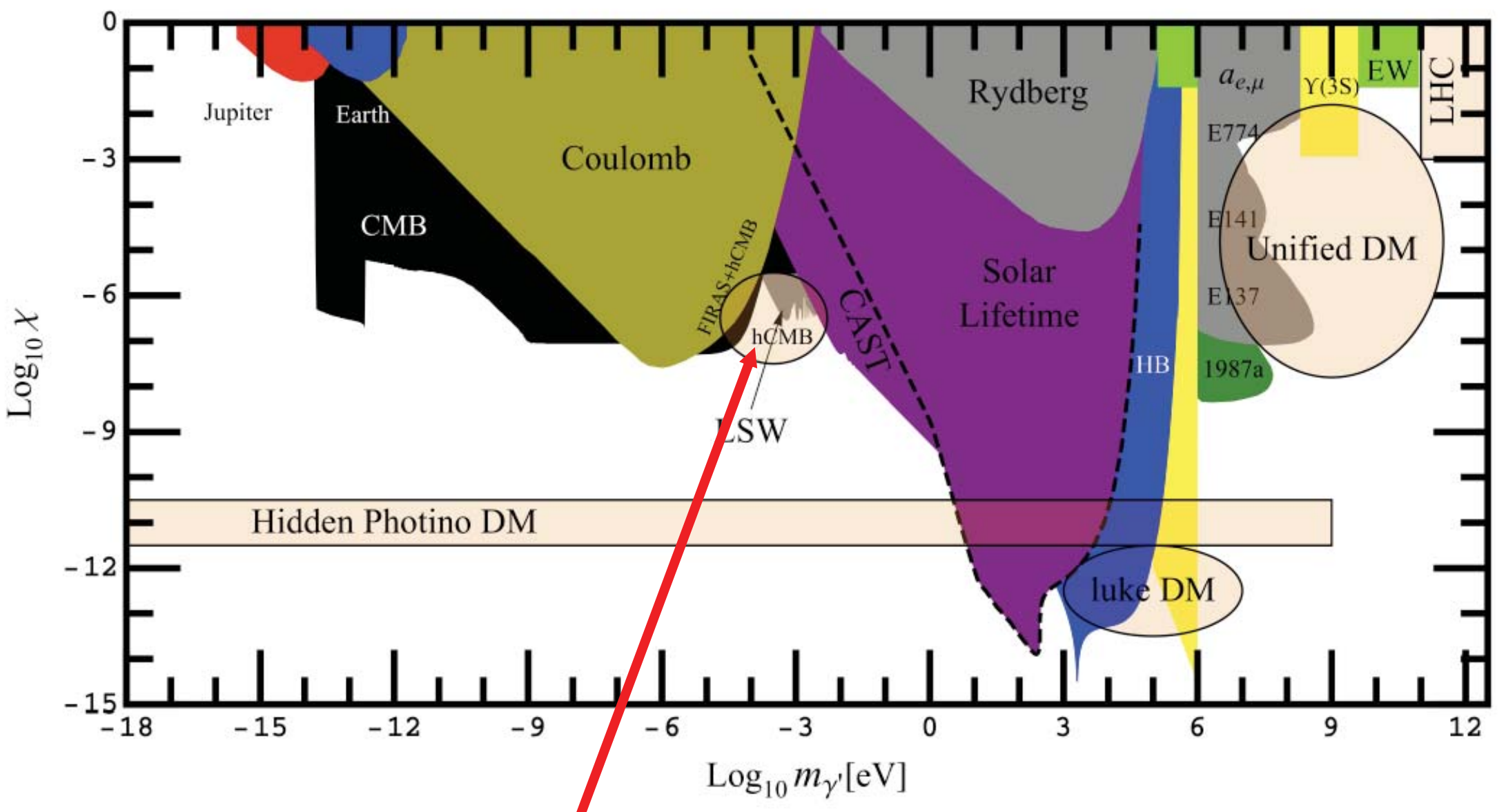

WMAP observes

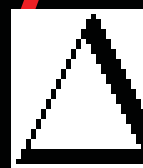




\section{More Hints...}

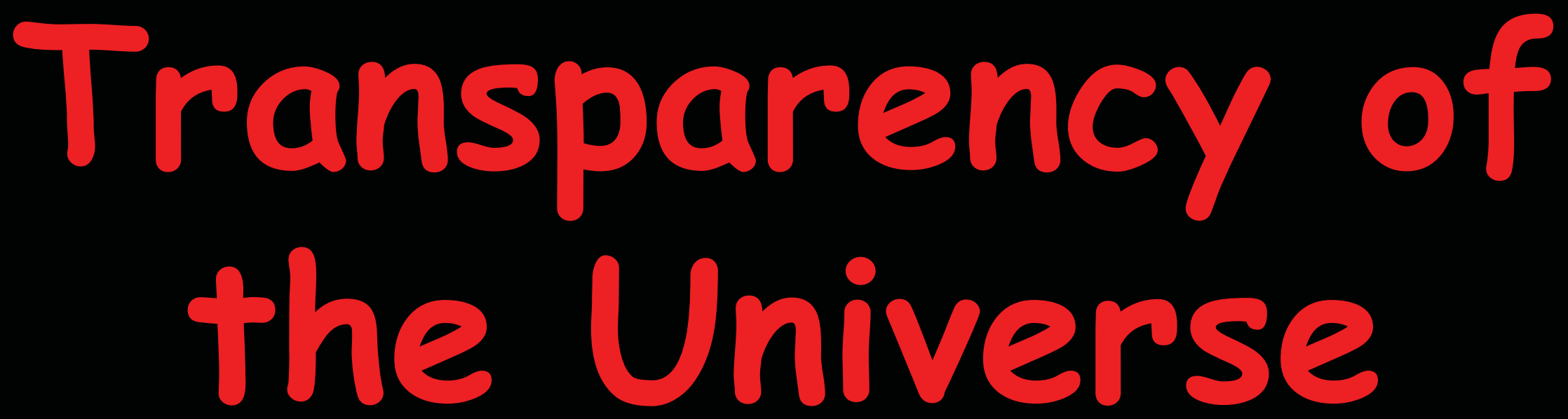




\section{The Universe should be quite opaque...}

- ... for high energy photons

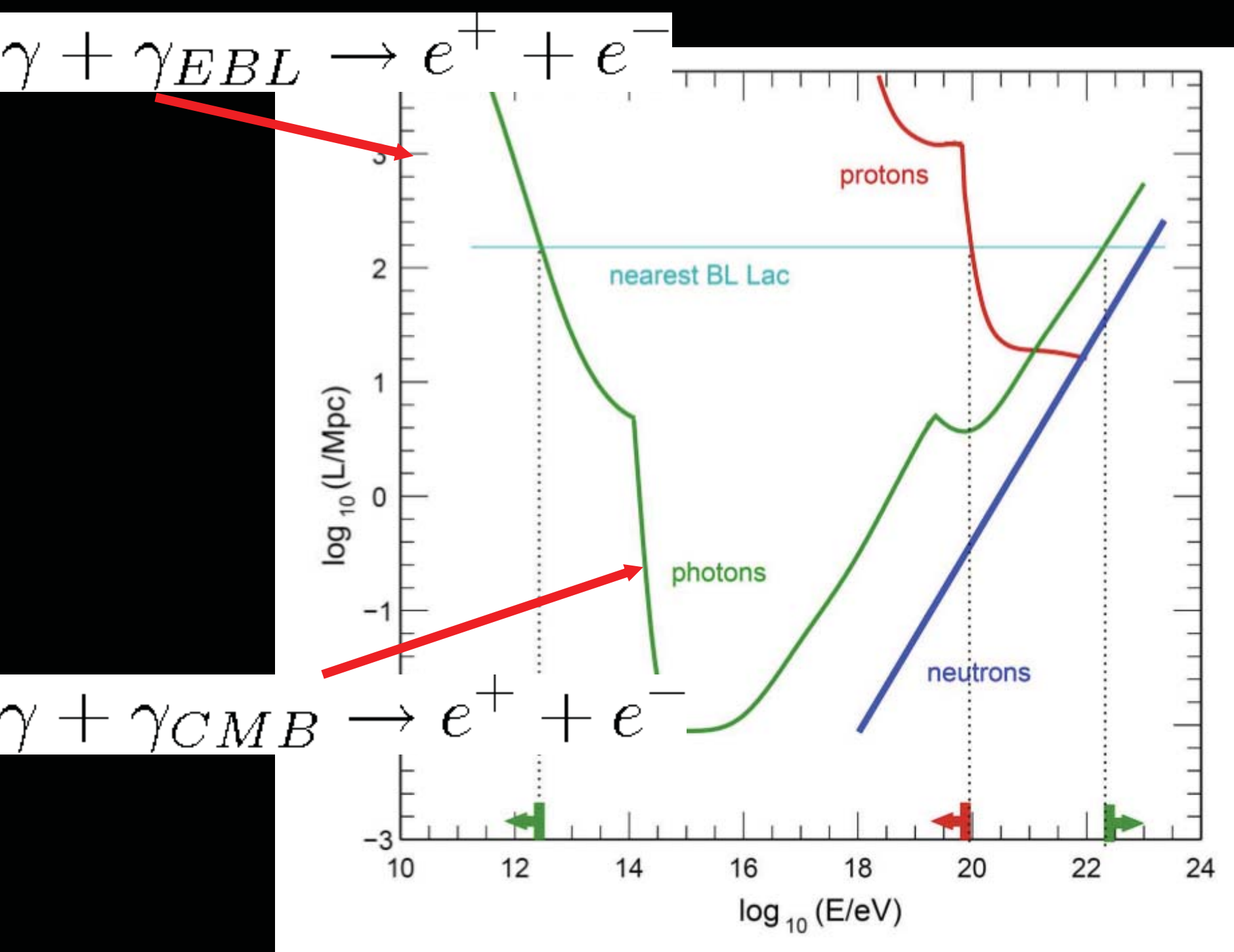




\section{From far away $\gamma$-ray source}

- Expect fewer high energy events!!

- Example $3 C 279$

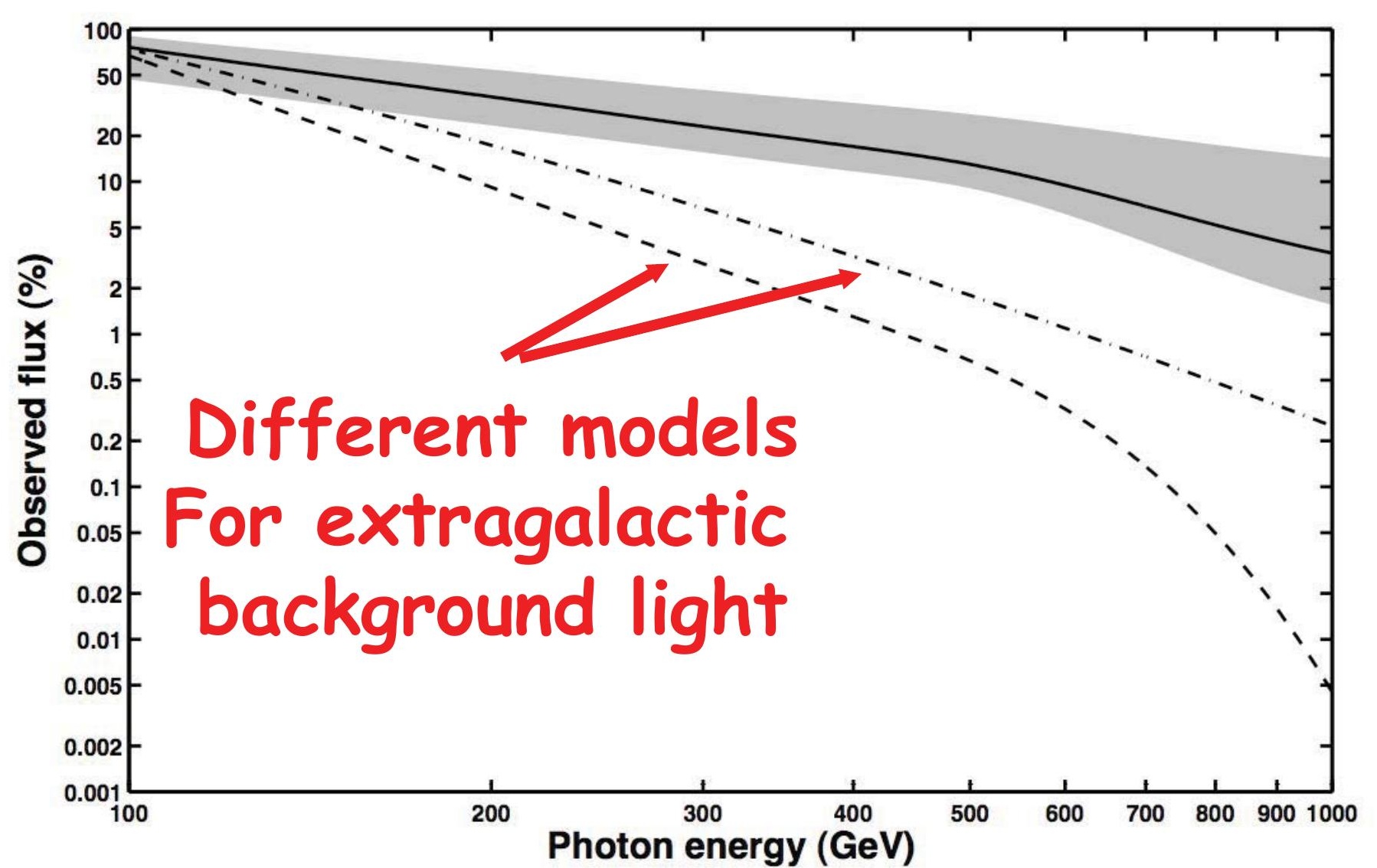


Observed...

- No such strong energy dependence!
$\mathbf{I P}^{3}$ น

University of Durham 


\section{Axion-like particles can help!}

- Photon oscillates into ALP

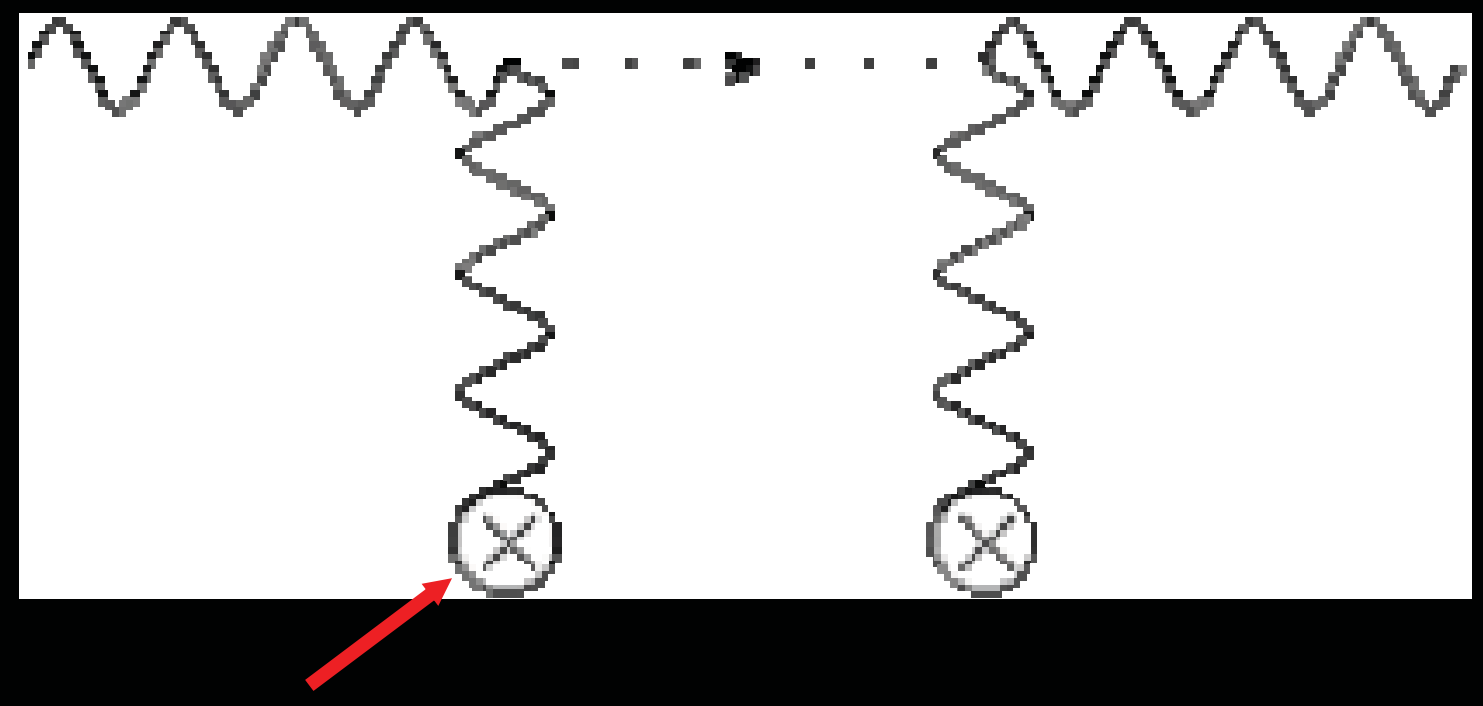

Intergalactig magnetic field 10-13 $\mathrm{T}$

- ALP doesn't see other photons

Not absorbed

Greater Transparency!! 


\section{ALPs help!}

- Example $3 C 279$

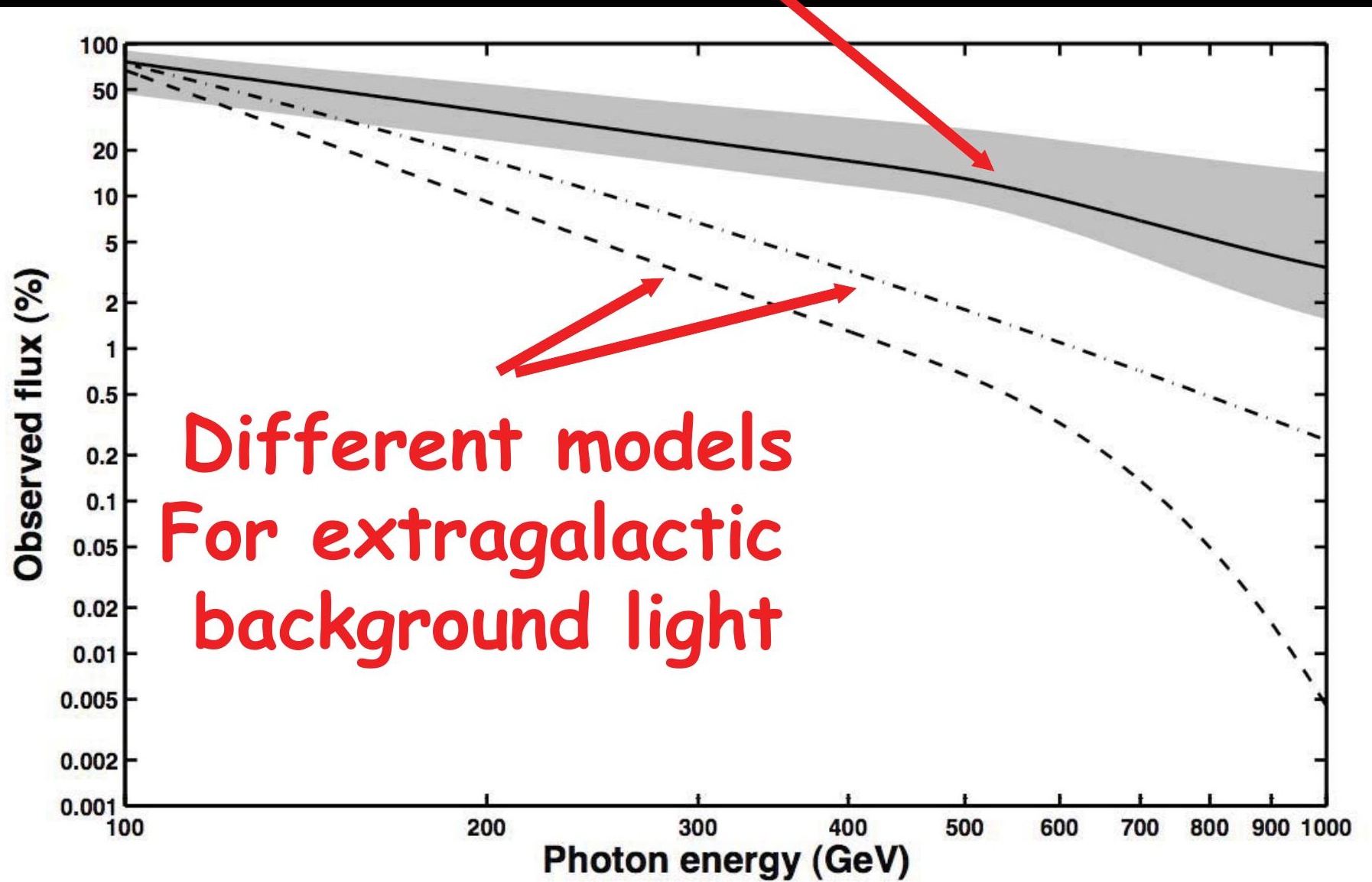




\section{Where are they???}

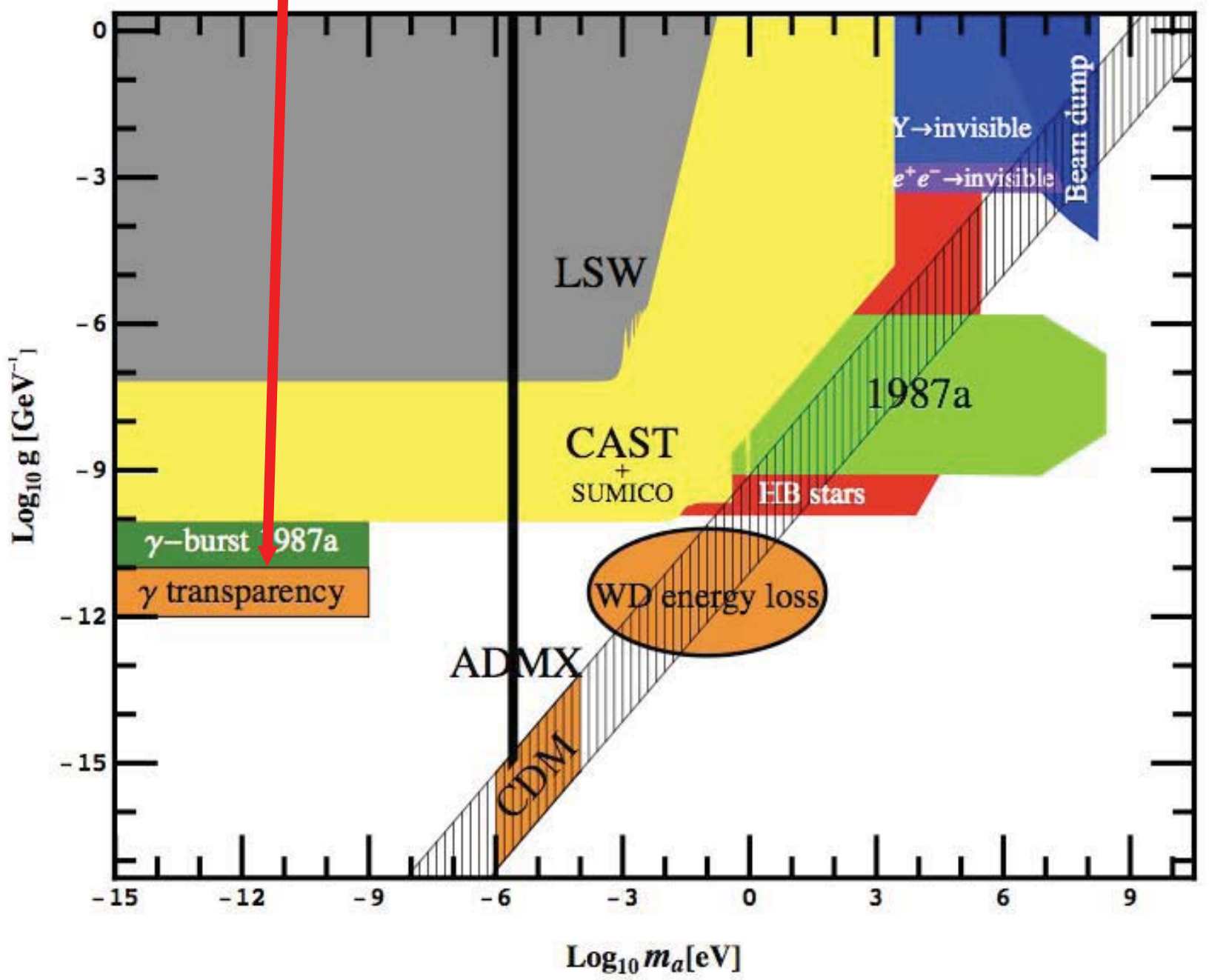


Summary 


\section{Summary}

- Astrophysics and Cosmology are a powerful probe of new light particles.

- Can test incredibly tiny interactions!!!

$\chi \sim 10^{-8}-10^{-14}, \quad, \epsilon \sim 10^{-14}, \quad g \sim \frac{1}{10^{10} \mathrm{GeV}}$

- Interesting hints for new particles!

- Not always perfectly understood!

Beware of uncertainties! 


$$
\begin{aligned}
& 3 . \\
& \text { Searching light } \\
& \text { particles in the Lab }
\end{aligned}
$$


Hints for new Physics

Model

Building

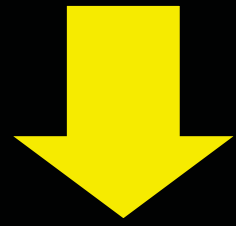

Bottom-up

(pheno)
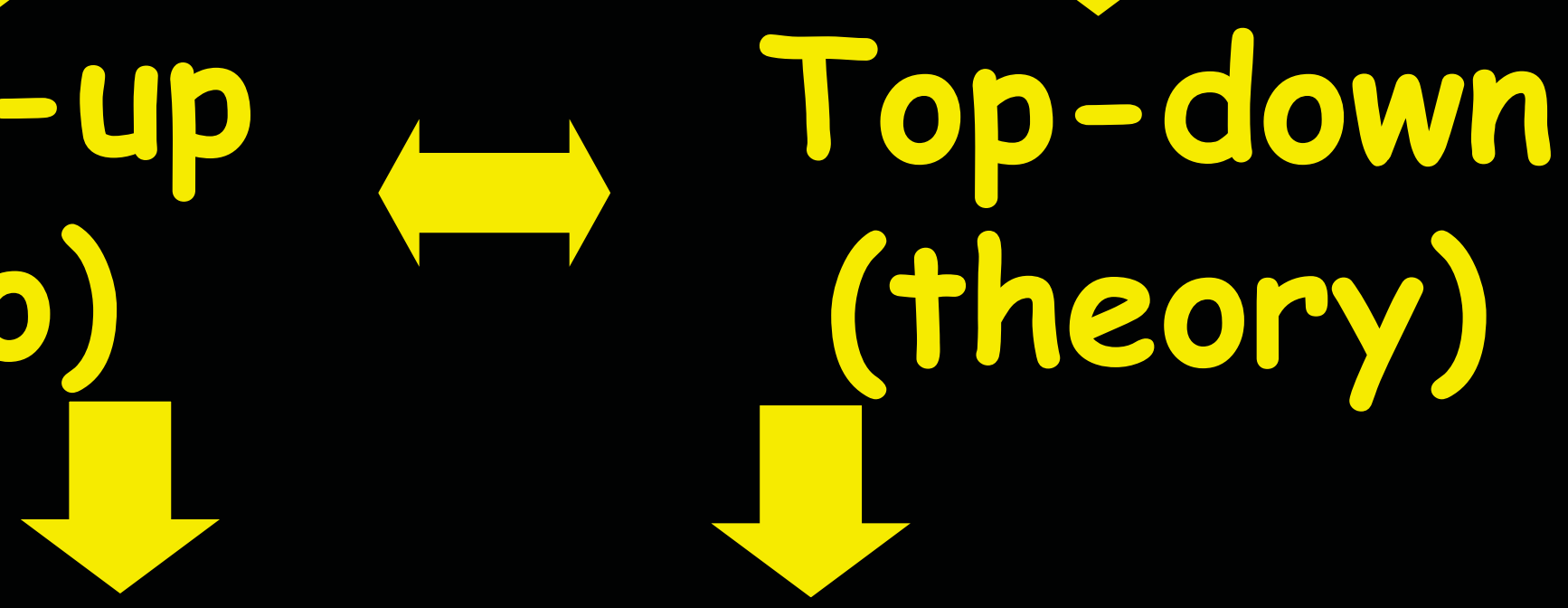

Observations/Experiments 


\section{Exploring fundamental high energy physics...}

- The direct approach: MORE POWER
LHC,
Tevatron
$+$
ILC,CLIC
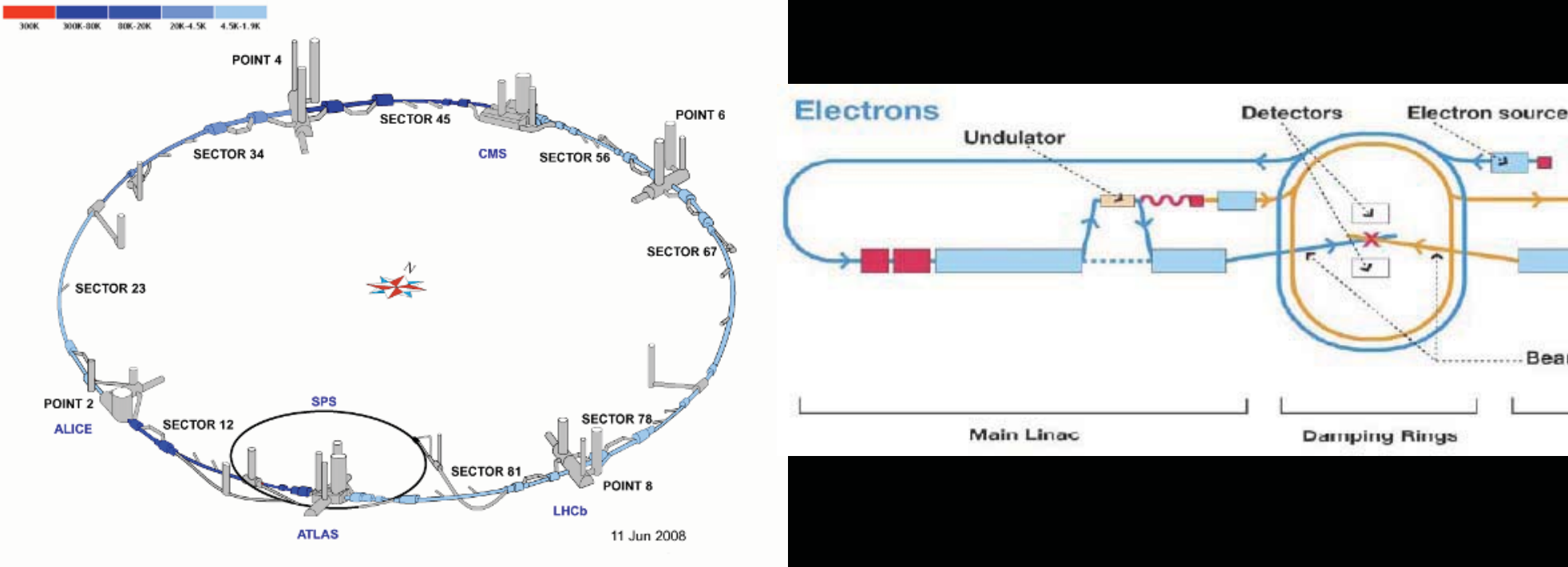

Positrons
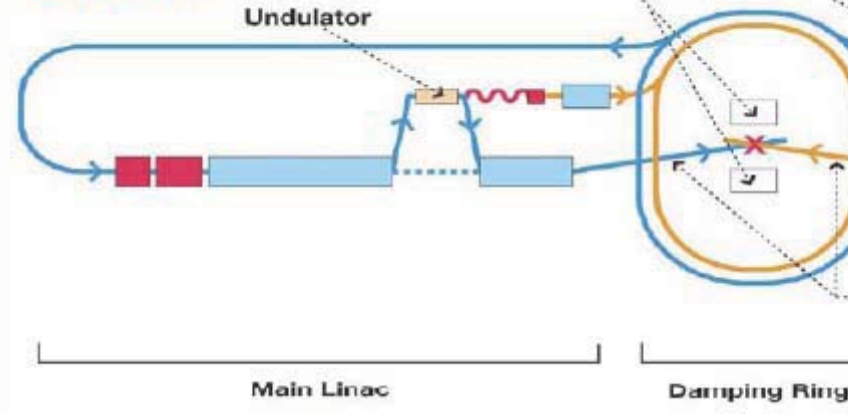

स'

$\square$

Beam delivery system

Main Linac

- Detects most things within energy range - E.g. may find SUSY particles, WIMPs etc. 


\section{But...}

- May miss very weakly interacting matter (Axions, WIMPs, WISPs...)

- Current maximal energy few TeV 


\section{Recycling...}

Complementary approaches 


$$
\begin{aligned}
& \text { Light shining } \\
& \text { through walls }
\end{aligned}
$$


"Light shining through a wall"

Laser
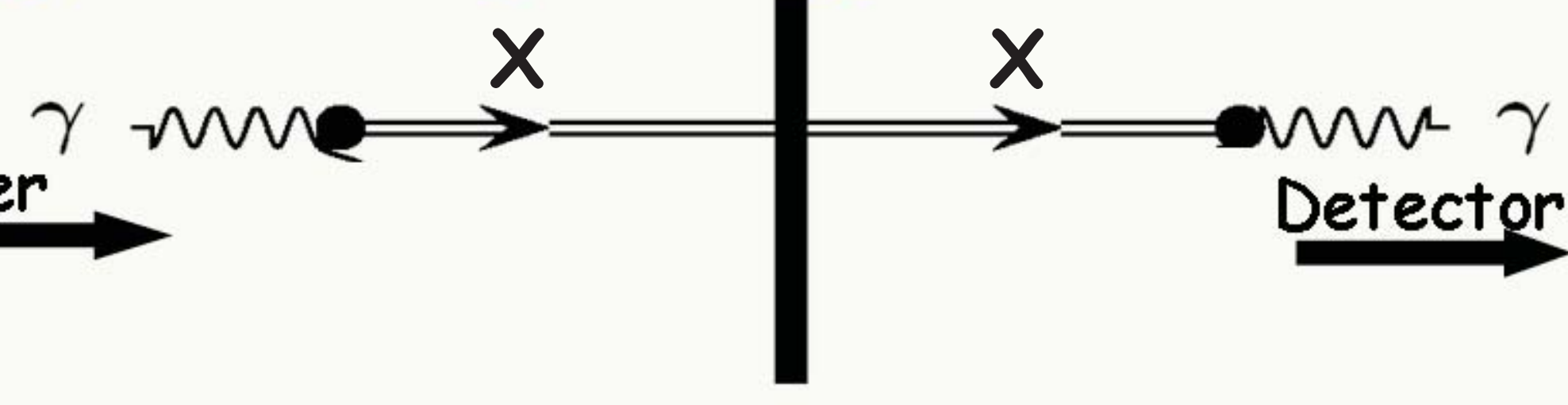
"Light shining through a wall"

Laser

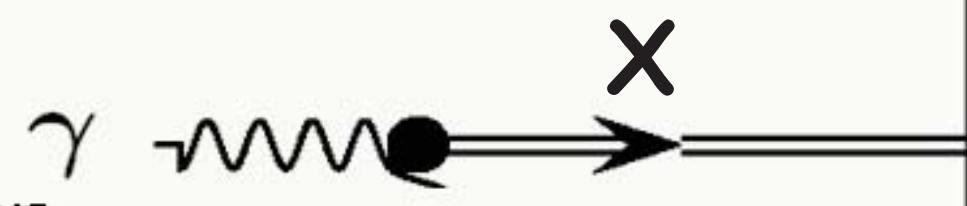

$10^{20} / \mathrm{s}$

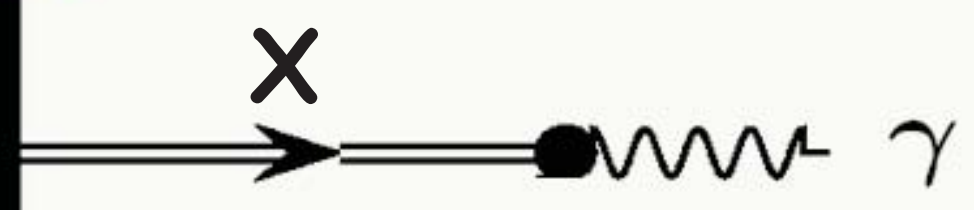

Detector

- Test $P_{\gamma \rightarrow X \rightarrow \gamma} \lesssim 10^{-20}$

- Enormous precision!

- Study extremely weak couplings! 


\section{Photons coming through the wall!}

- It could be Axion(-like particle)s!

- Coupling to two photons: $\frac{1}{M} a \tilde{F} F \sim \frac{1}{M} a \overrightarrow{\mathbf{E}} \cdot \overrightarrow{\mathbf{B}}$
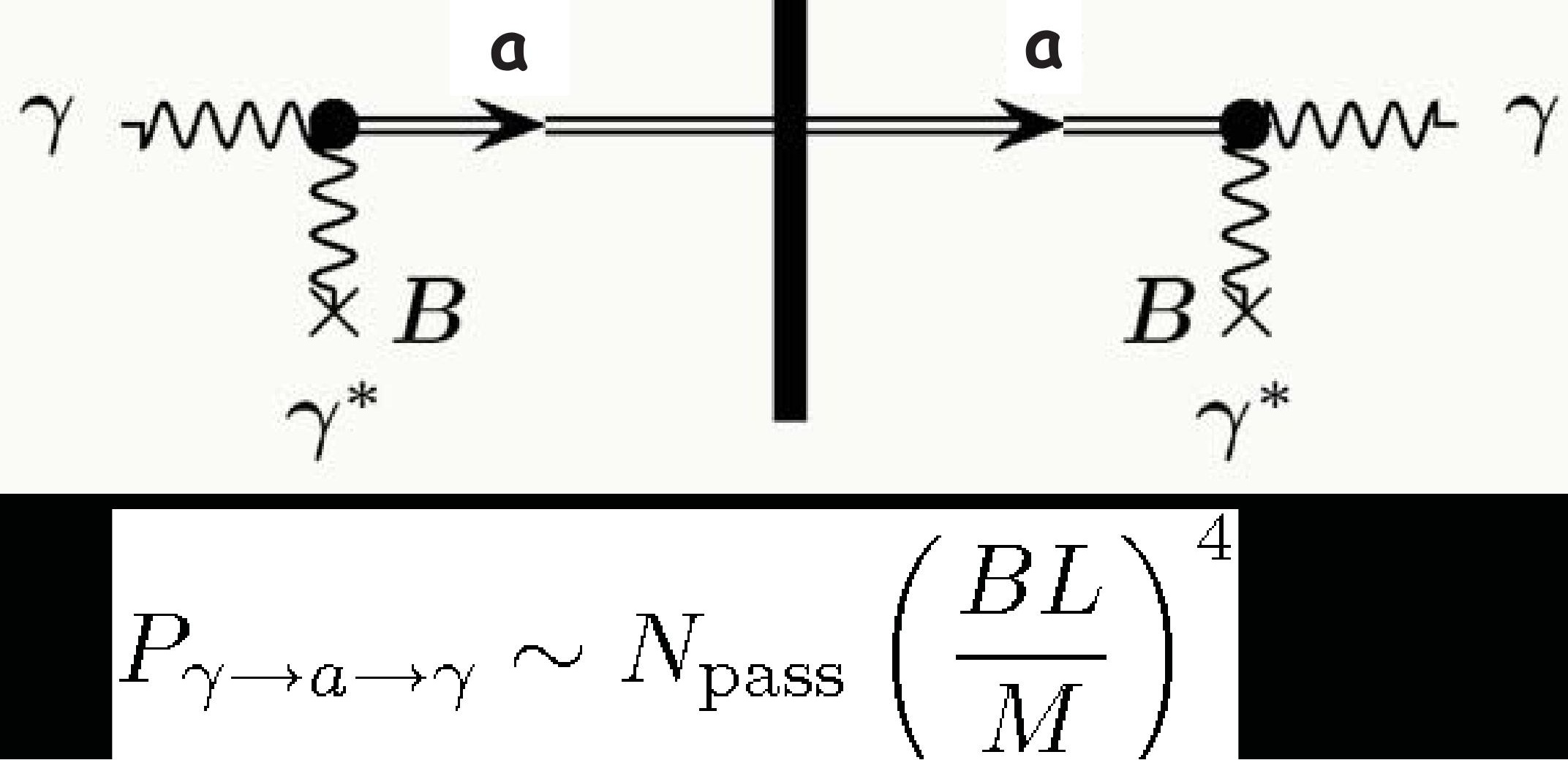


\section{Light Shining Through Walls}

- A lot of activity

- ALPS

- BMV

- Gamme $25 \mathrm{~cm}$

- LIPPS

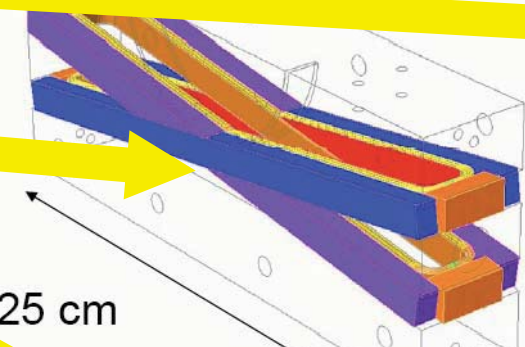

- OSQAR

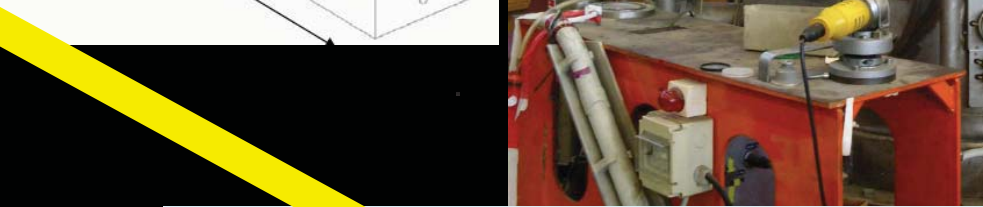

$(10)^{2}$
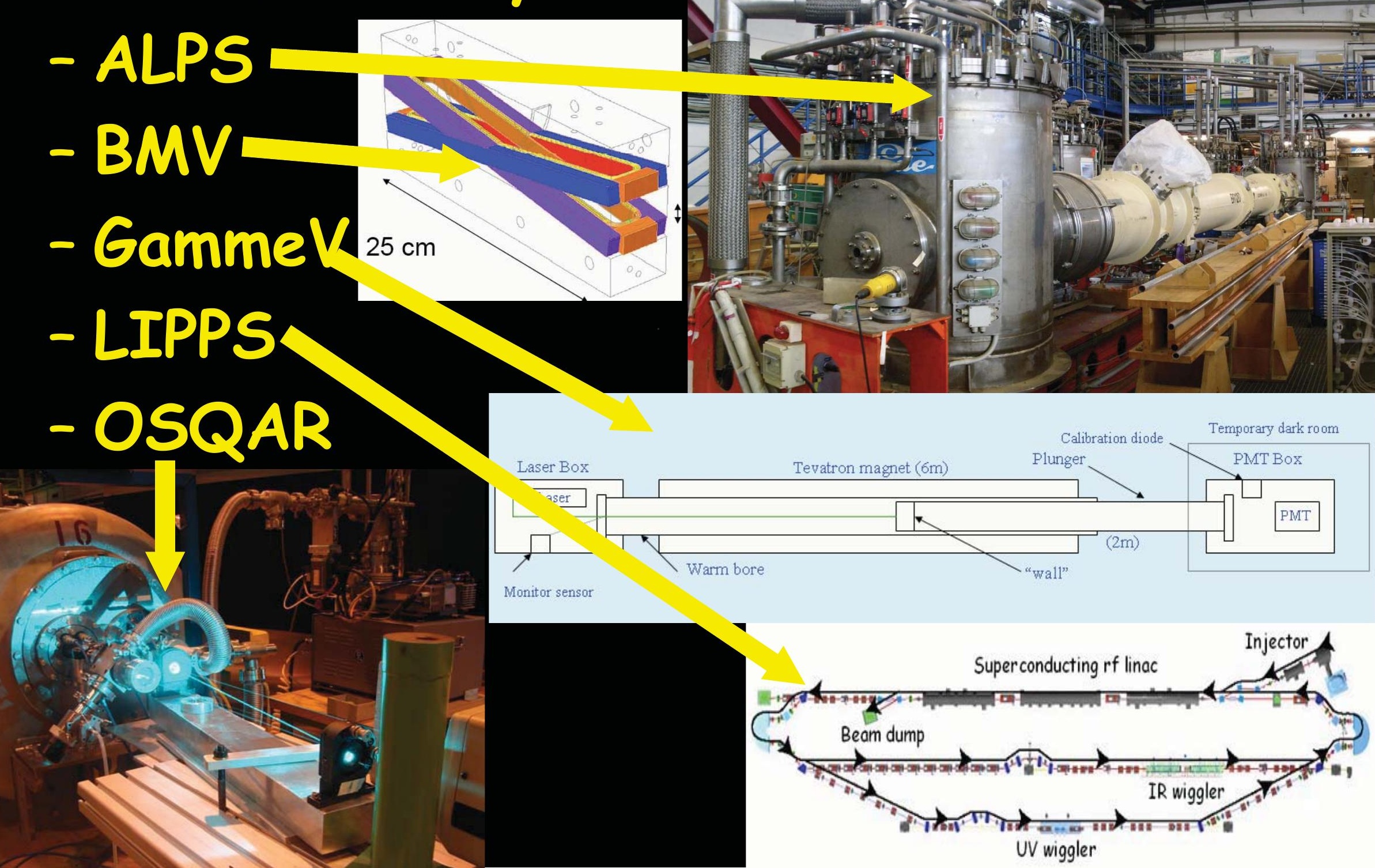


\section{Small coupling, small mass}

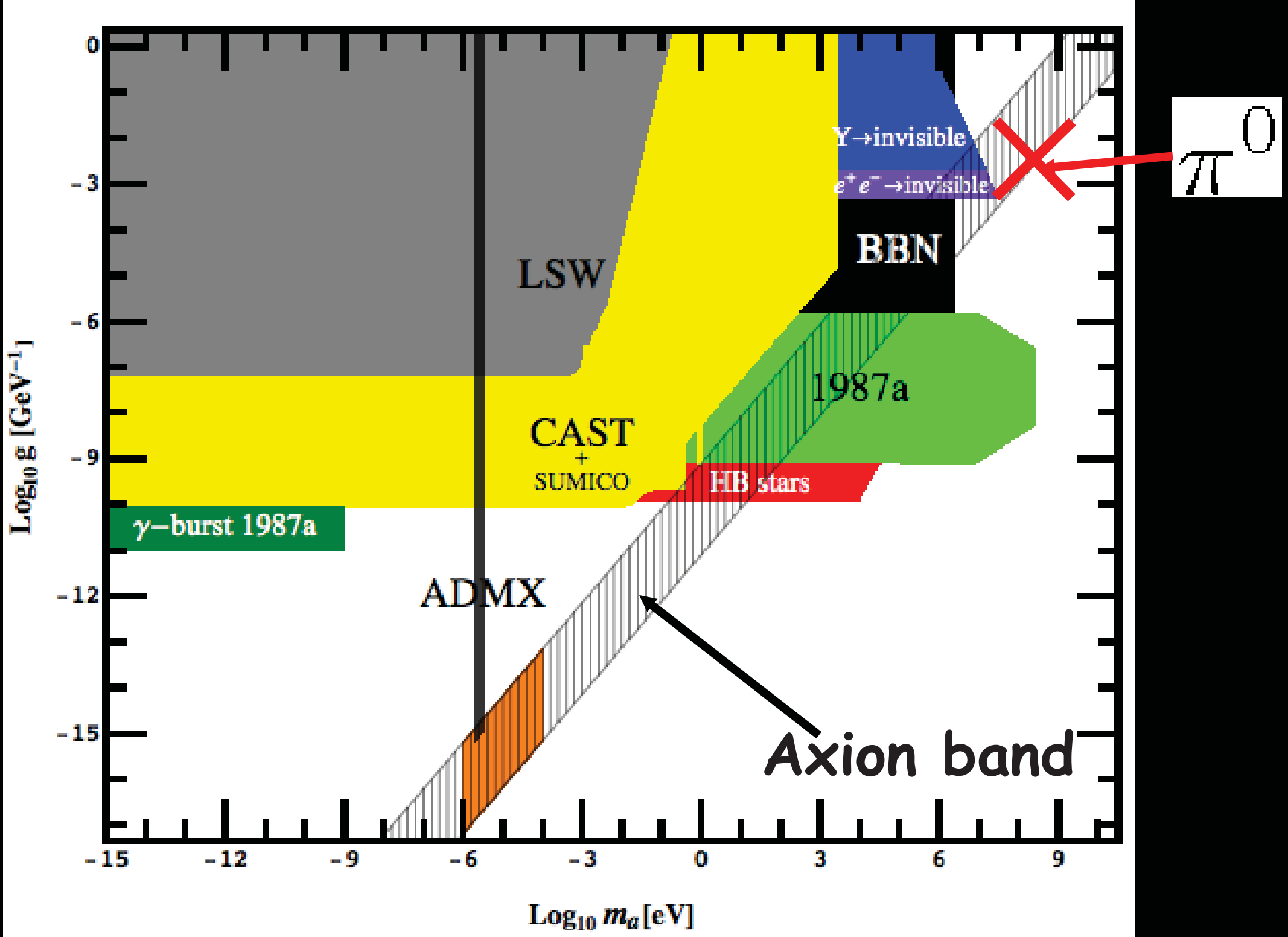


WISPS=Weakly interacting sub-eV particles

- Axions

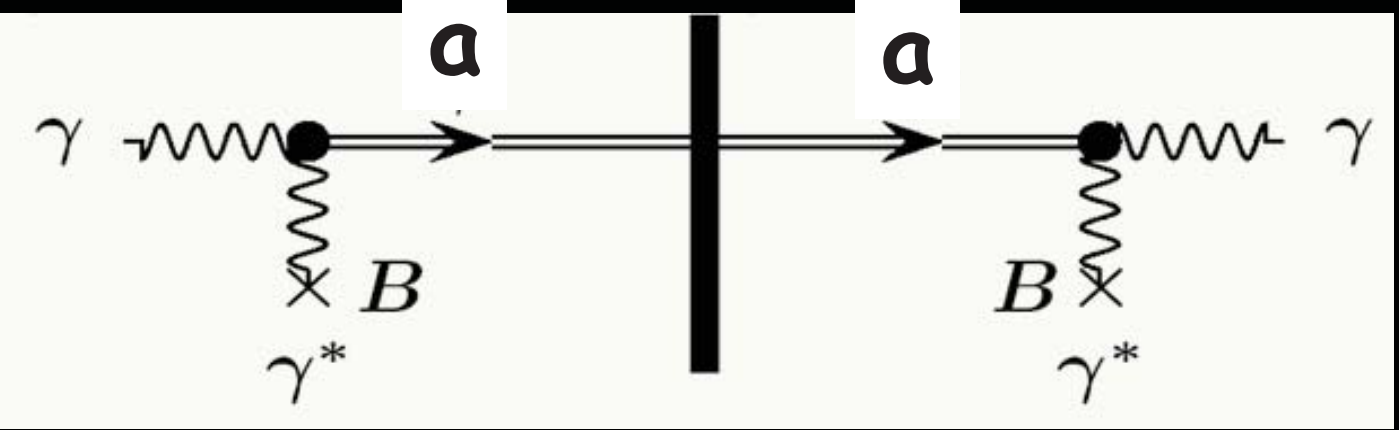

- Massive hidden photons

(without B-field)

$=$ analog $v$-oscillations

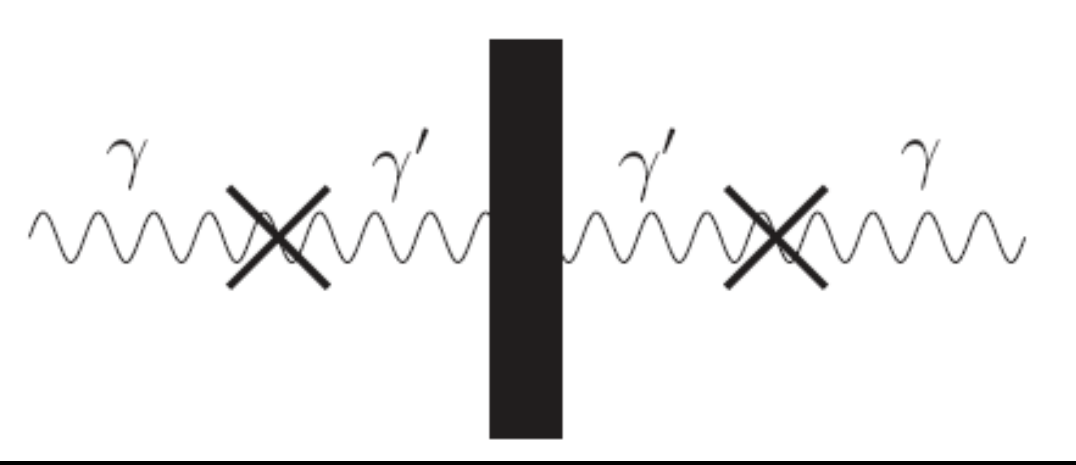

- Hidden photon + minicharged particle (MCP)

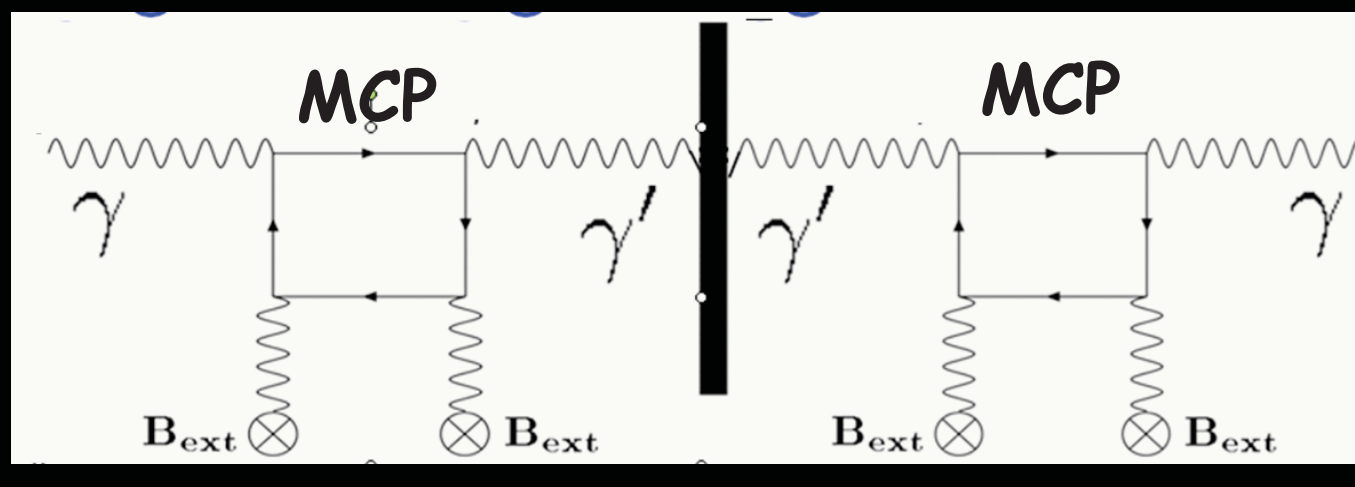




\section{Hidden Photons}

$m(1 P 3 n$

\section{LSW already competitive + testing interesting area}

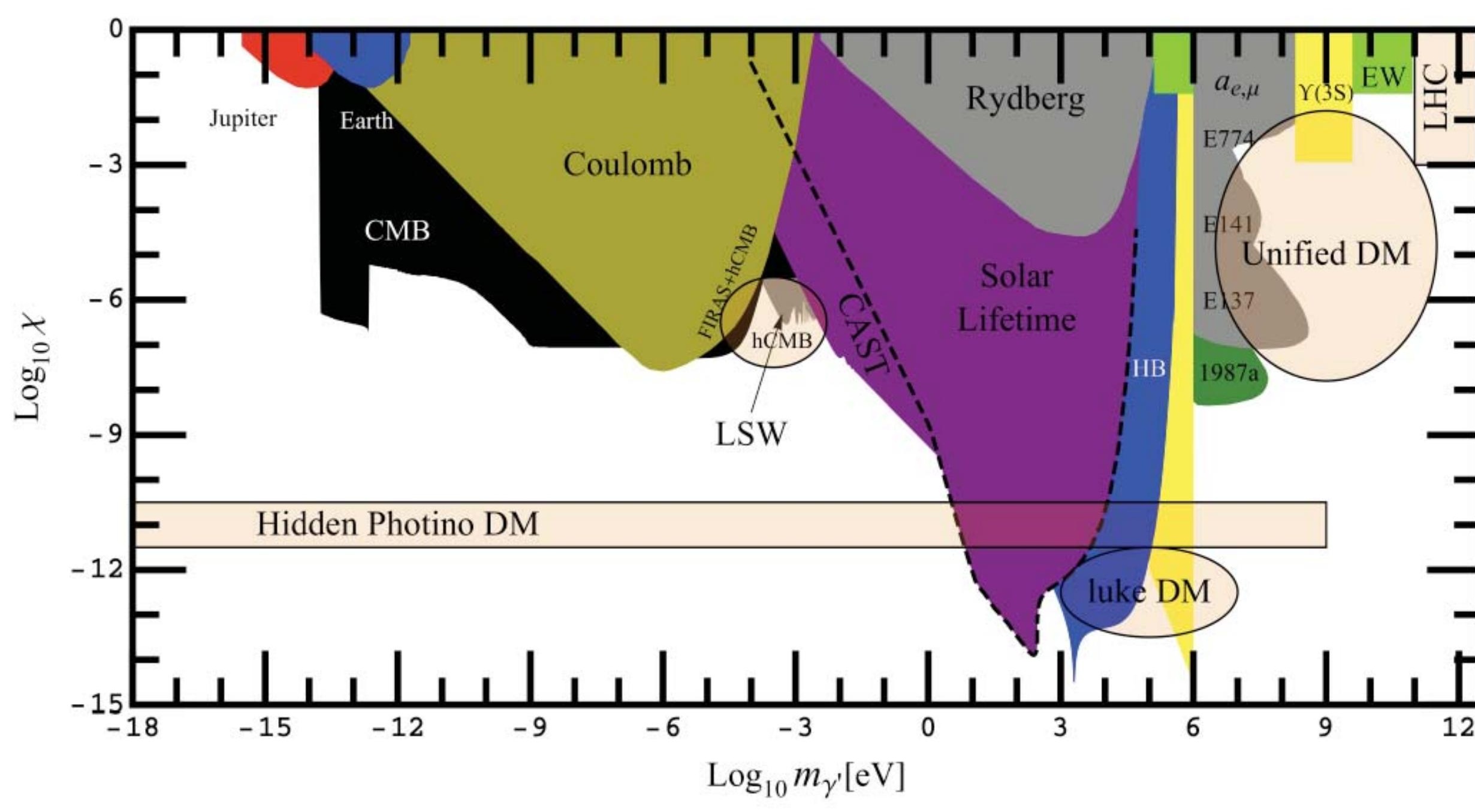




\section{Coincidences?}

- Neutrino masses:

$$
m_{\nu} \sim \mathrm{meV}
$$

- Scale of dark energy:

$$
\rho_{\Lambda} \sim(\mathrm{meV})^{4}
$$

- Energy density of the Universe:

$$
\rho_{\text {today }} \sim(\mathrm{meV})^{4}
$$




\section{Hidden Photons}

LSW already competitive + testing interesting area Dark energy scale

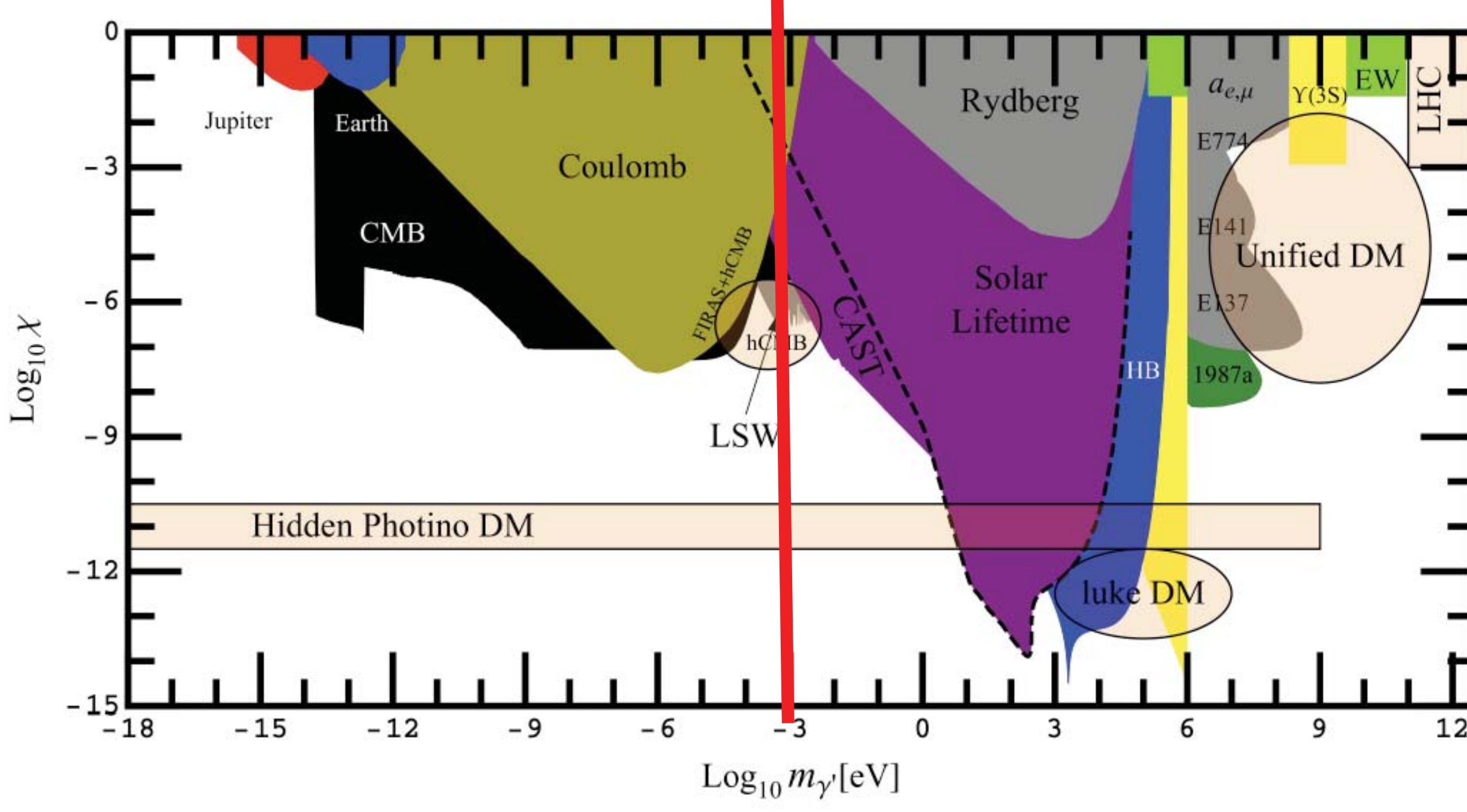




\section{Something to hide?}




\section{Something to hide?}

Use Hidden Photons (

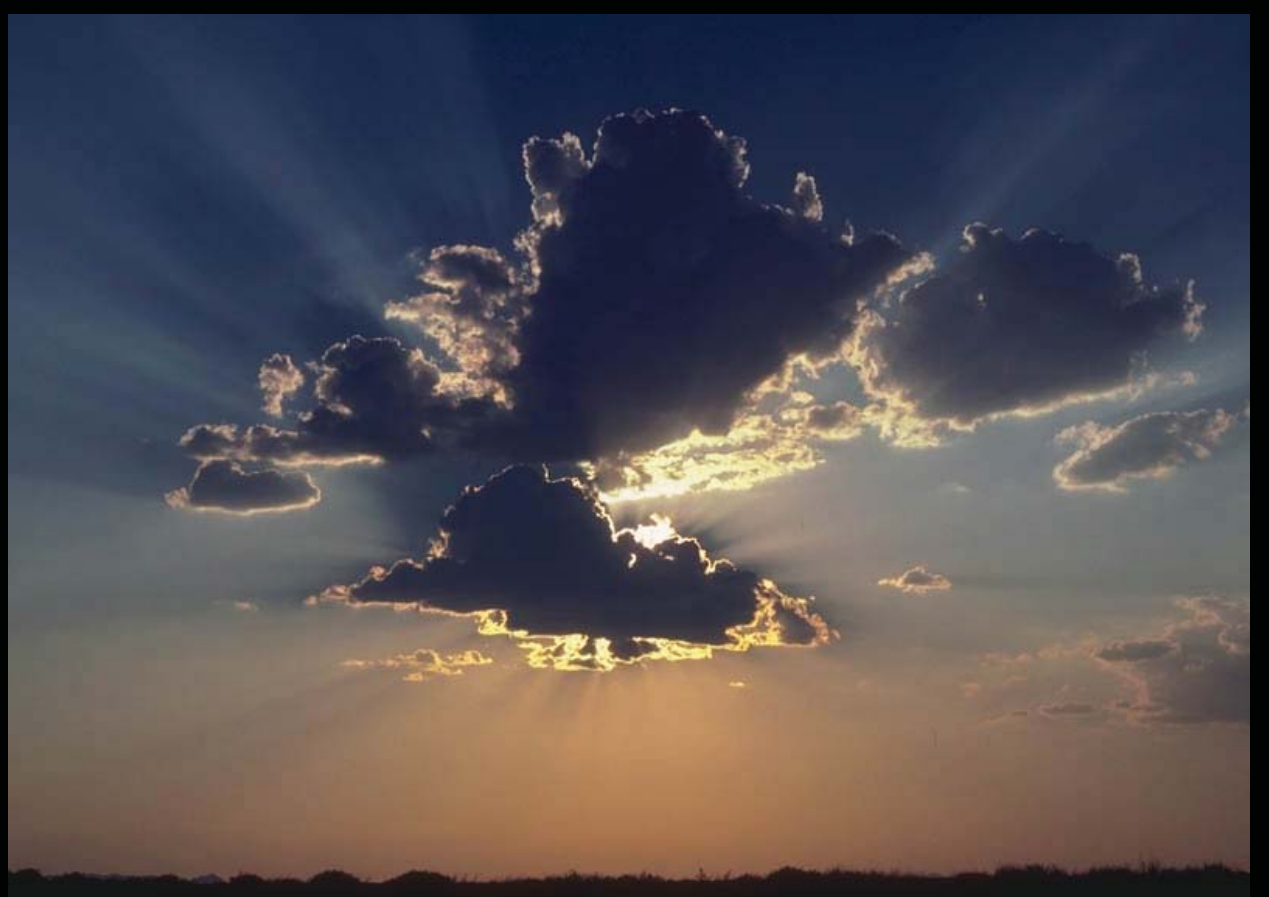

to communicate! 


\section{Practical applications :-)}

- Communicating through the Earth

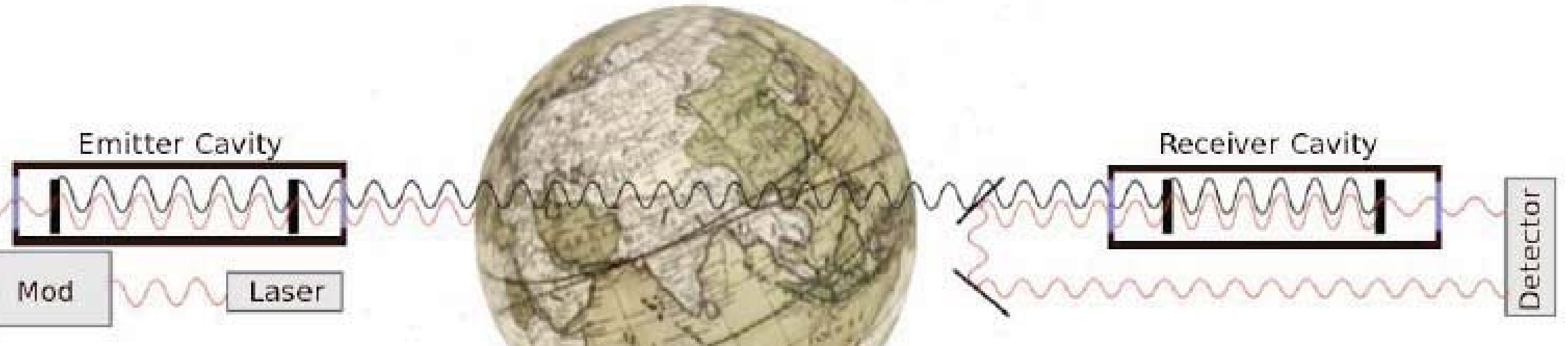




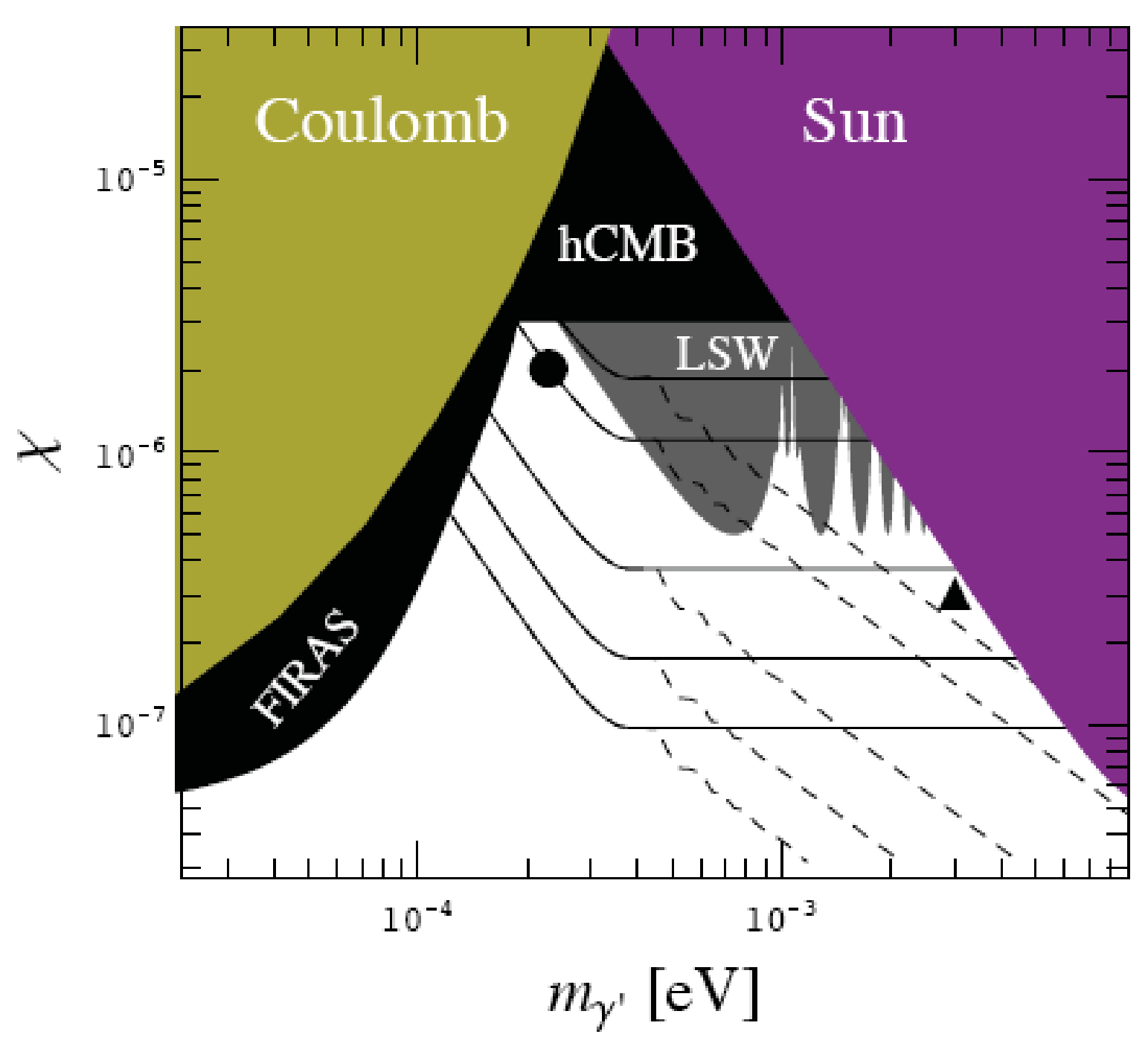




\section{A cavity \\ experiment}


It's a Light shining through walls clone

"Light shining through a wall"
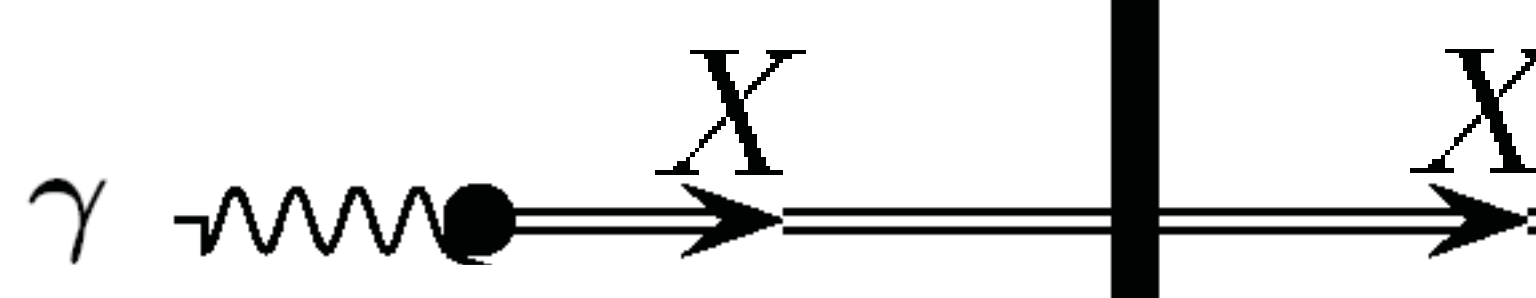

Emitter cavity

- Microwaves instead of laser

Detector cavity 


\section{Setup}

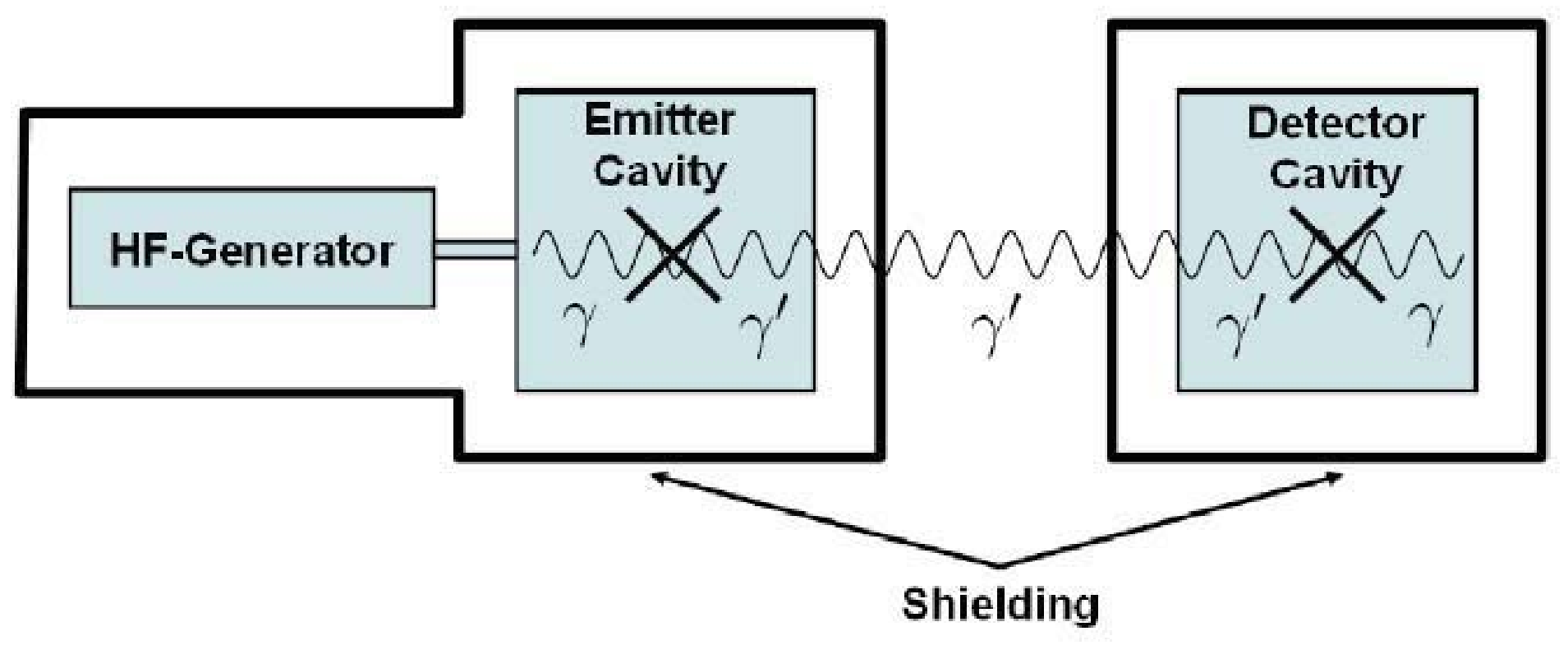




\section{Advantages}

- Resonant cavity setup: Cavity in production and regeneration region

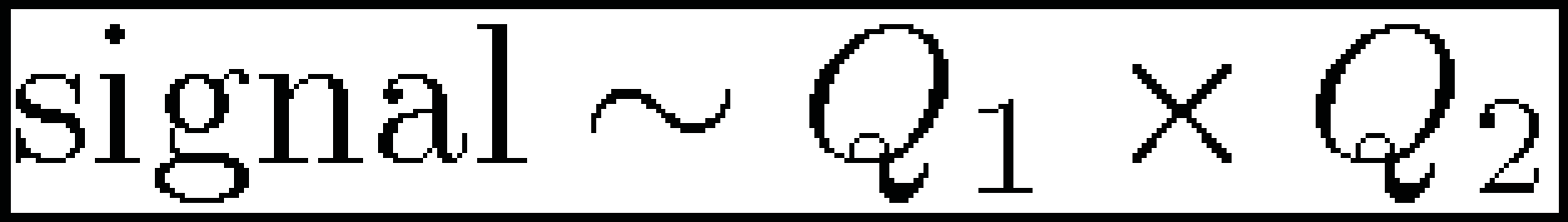

- Microwave cavities can have very high Q-factors 1011!

- Sensitive to masses in the interesting $\mu \mathrm{eV}$-meV range 
Sensitive to variety of WISPs

- Axions

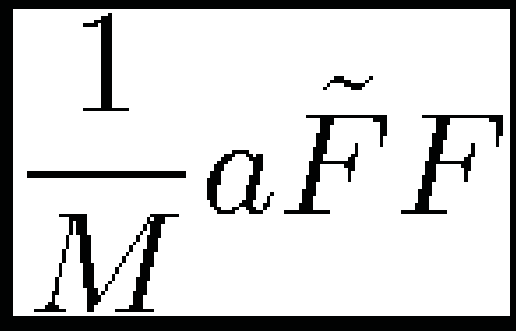

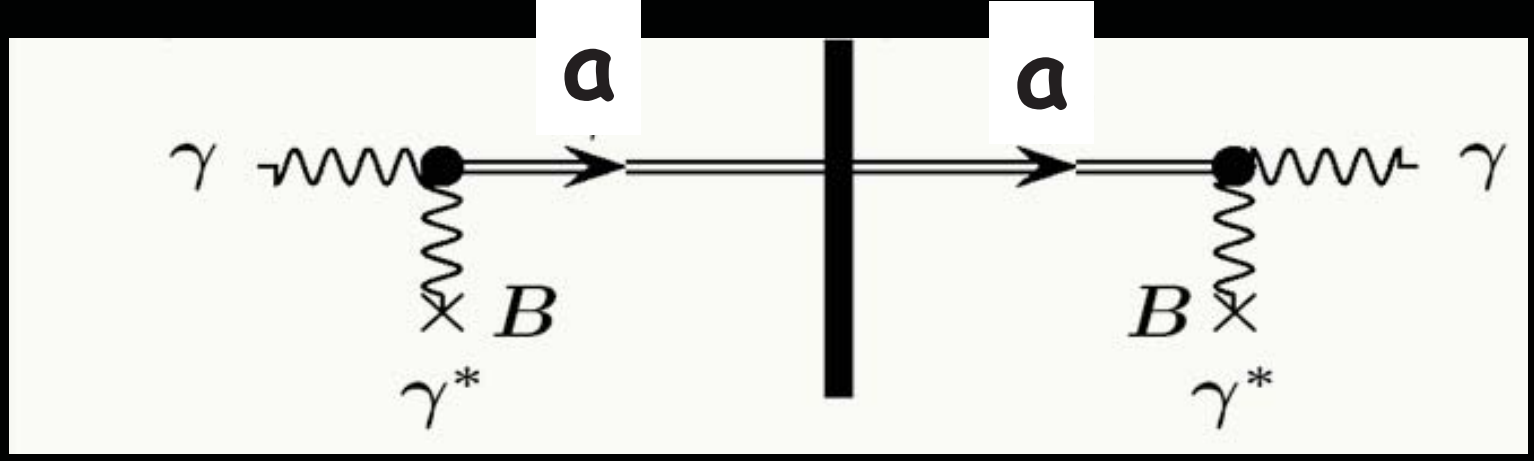

- Massive hidden photons (without B-field) $=$ analog $v$-oscillations

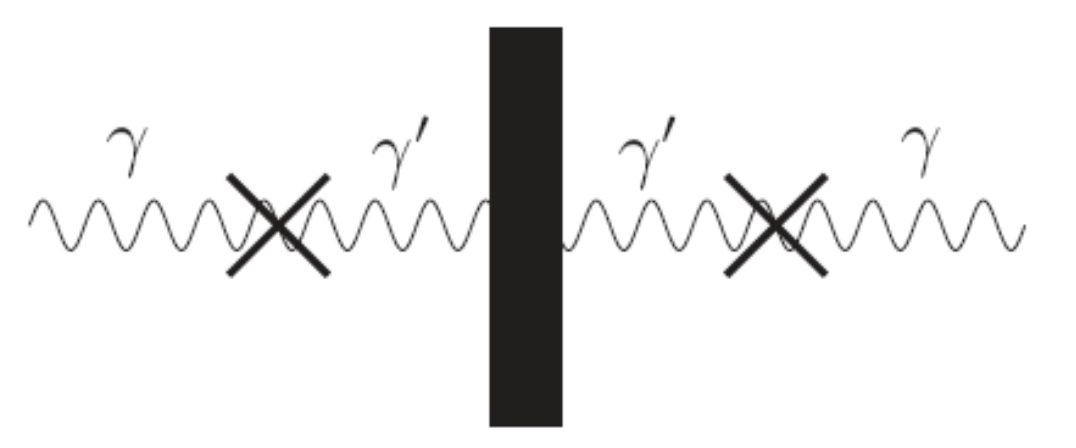

- Hidden photon + minicharged particle (MCP)

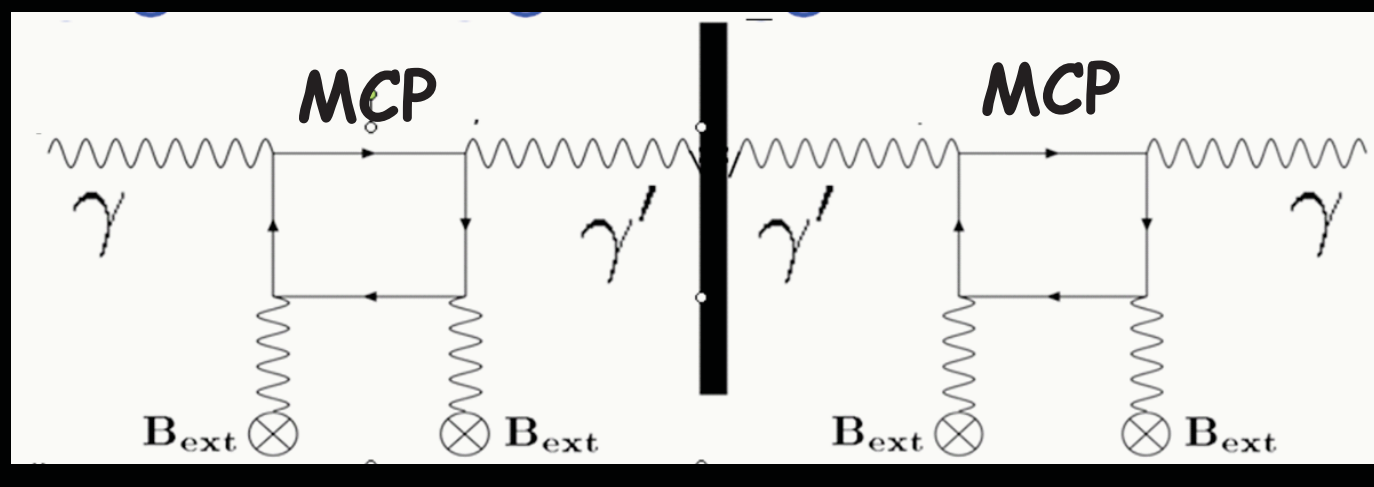




\section{Impressive sensitivity/discovery potential}

\section{Hidden photons}

Axion (-like partice)s
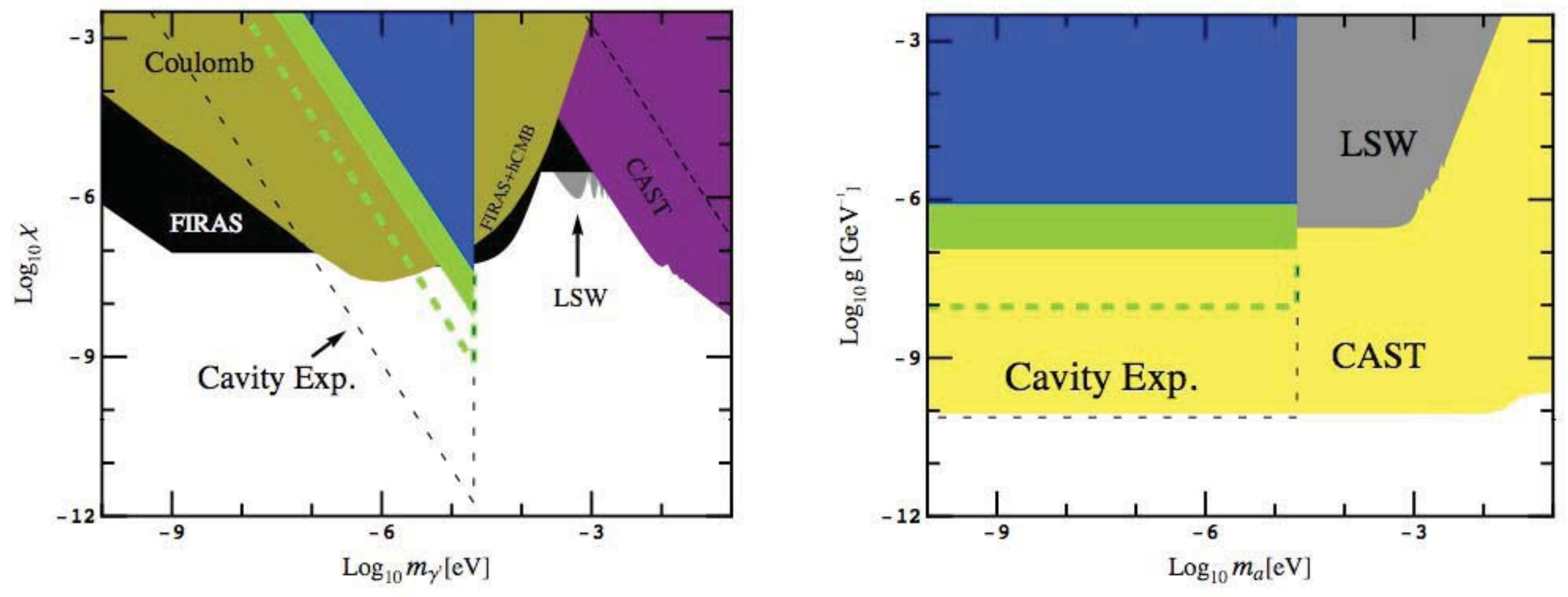
Impressive sensitivity/discovery potential

$m(1 P 3) m$

University of Durham

\section{Hidden photons}

\section{Axion (-like partice)s}
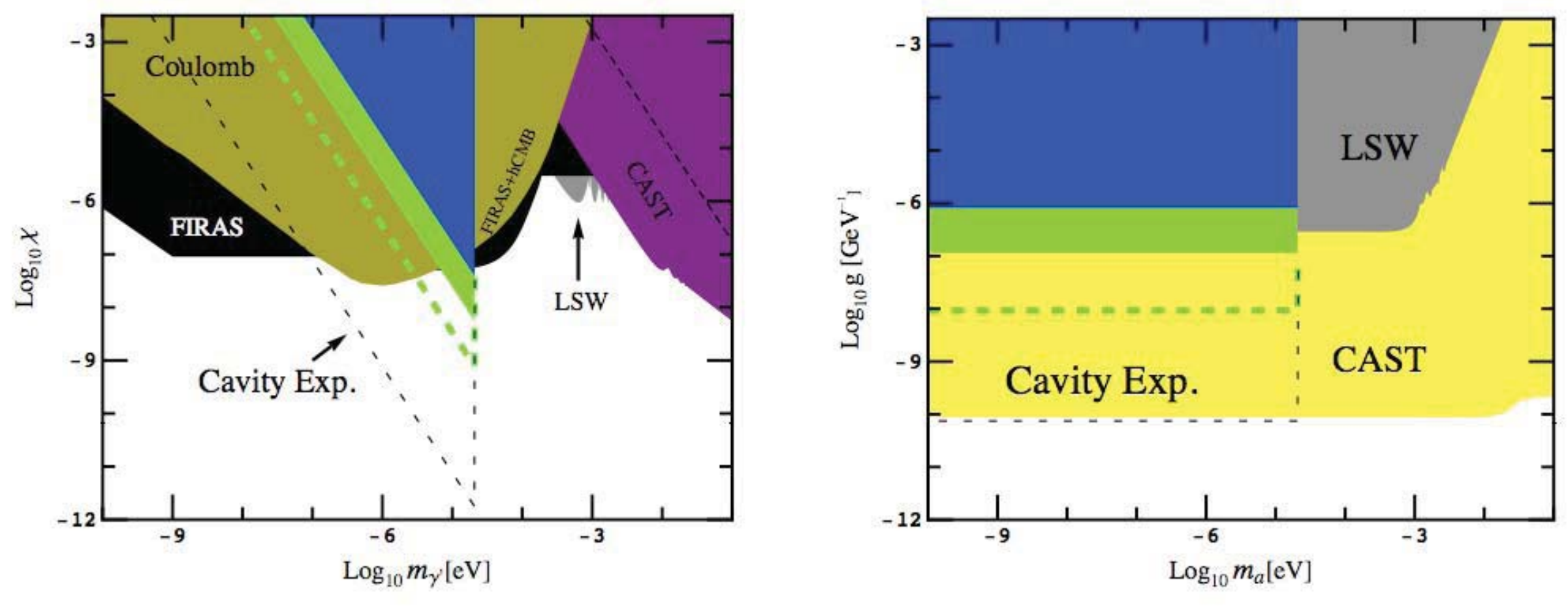

Experiments are underway:

@ UWA Perth

@ Yale 


\section{An aside:}

\section{Sub-Quantum interference}

J. Hartnett, JJ, R. Povey and M. Tobar 1101.4089 


\section{Resonant regeneration}

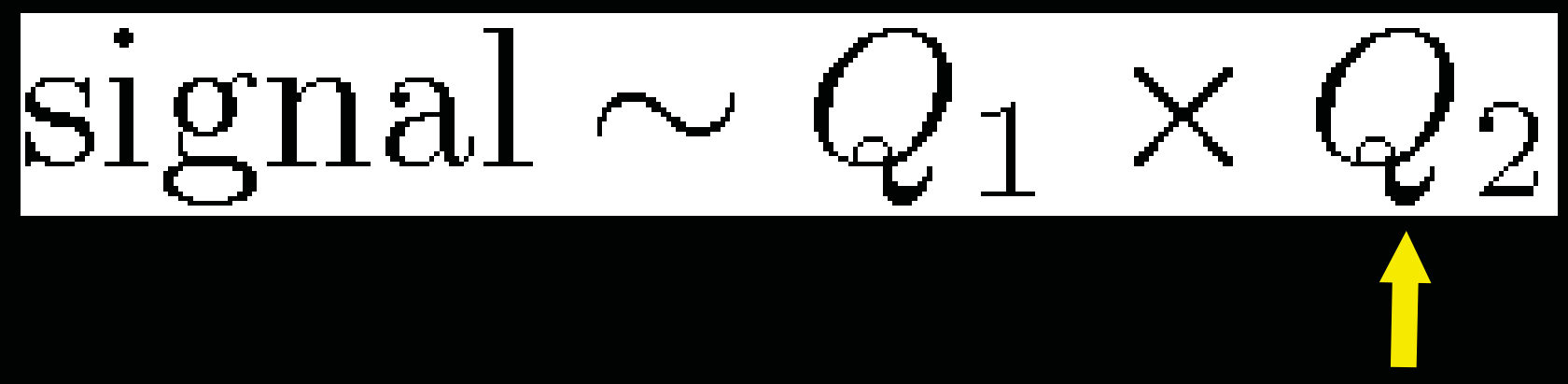

detector cavity

- The detector acts as high quality oscillator driven by (small) external force (ALPs, HPs...)

- Amplification because

the photon amplitude adds up in each pass 


\section{Less than one photon inside cavity...}

- Already at relevant power levels

$$
\begin{aligned}
P_{\text {out }} \sim P_{\text {loss }} & =\omega \frac{E_{\text {stored }}}{Q}=\omega \frac{\hbar \omega}{Q} \\
& \sim 2.6 \times 10^{-19} \mathrm{~W}\left(\frac{f}{\mathrm{GHz}}\right)^{2}\left(\frac{10^{5}}{Q}\right) \gg 10^{-23} \mathrm{~W}
\end{aligned}
$$

- Even more relevant for optical experiments (higher frequency)

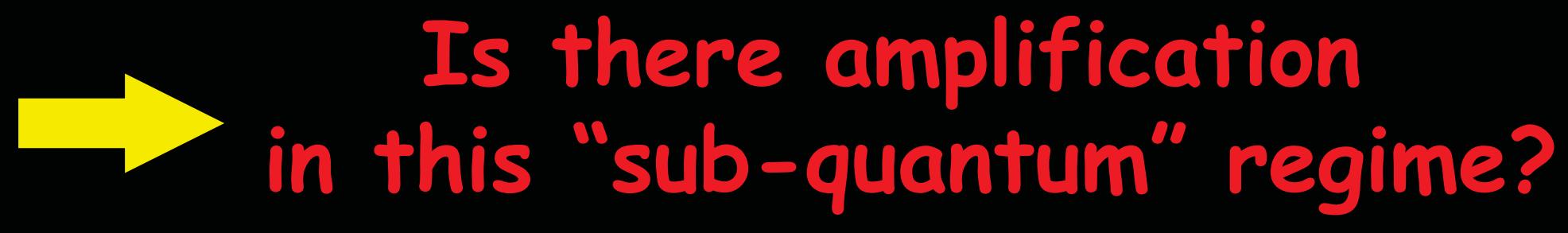


A test/demonstration setup...

\section{Input power(f)}

\section{Cavity}

Contains less

\section{I}

tiny input power (simulate axion signal)

\section{Detector}

- Do we see the (fractional) photon interfering with itself?

(i.e. amplitudes adding up) 


\section{A true measurement...(preliminary!)}

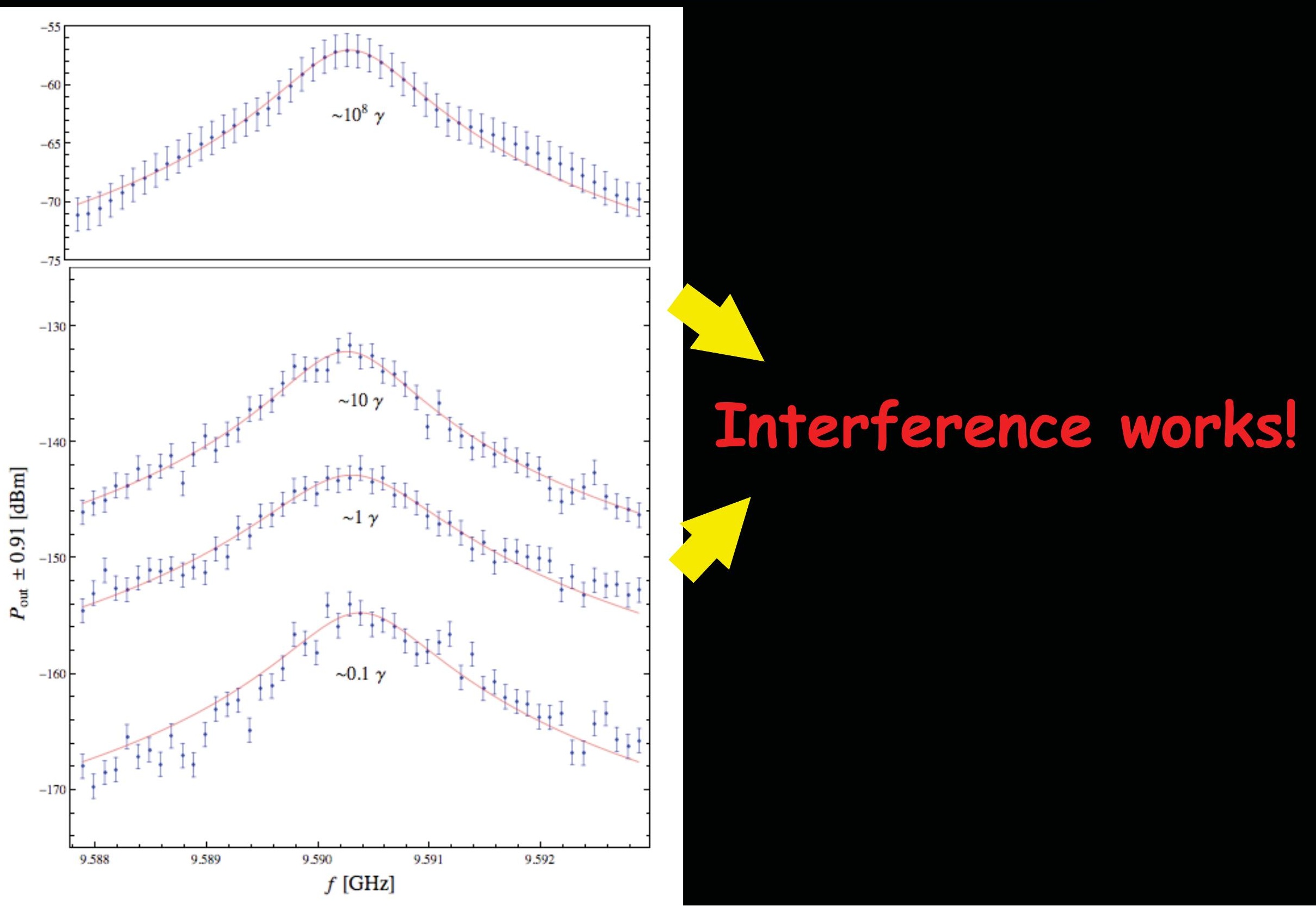


Laser Polarization Experiments 


\section{Dichroism (Rotation)}

$m(1 / P 3)$

University of Durham

- Coupling

$$
\mathcal{L} \sim g a F^{\mu \nu} \tilde{F}_{\mu \nu} \sim g a \vec{E} \cdot \vec{B}
$$

B $_{\text {Ext }}$

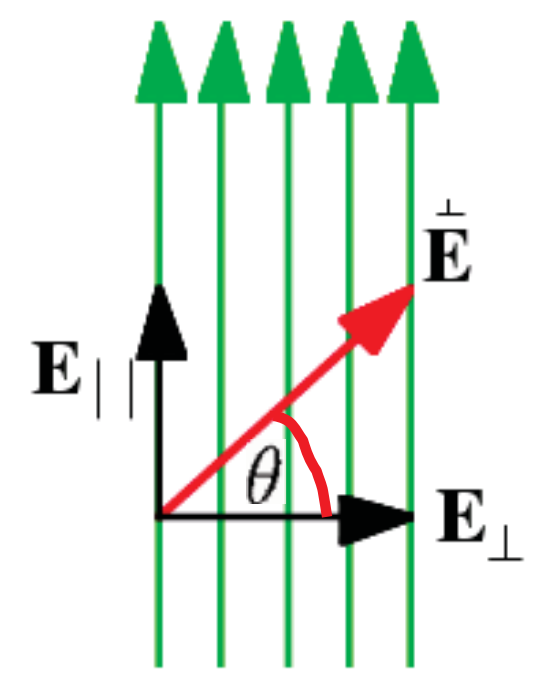

before $\mathrm{g}_{\mathrm{p} \gamma}$

WWWM

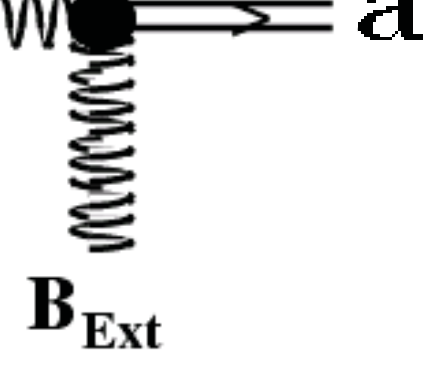

B Ext $_{\text {E }}$

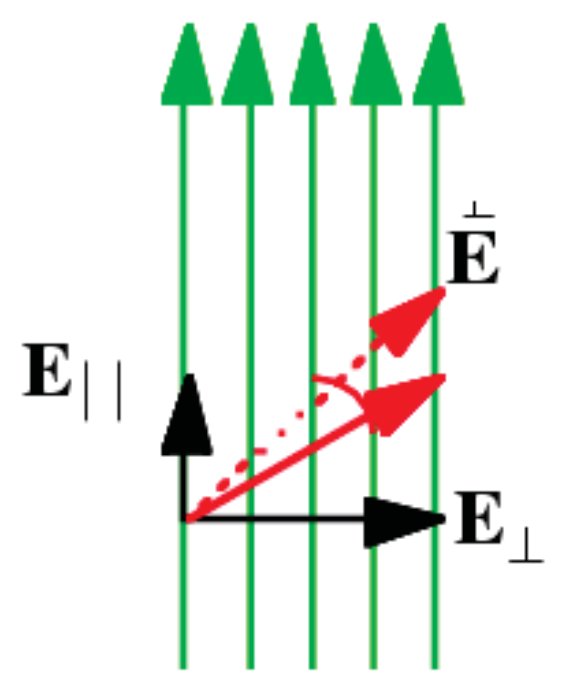

after

\section{$\underline{\text { rotation } \varepsilon}$}

F. Brandi et al. hep-ex/0006015

\section{$\sin (2 \theta)$}




\section{Ellipticity}

$m \mathbb{1}^{3} \mathbf{P}^{2} n$

\section{Virtual ALP production leads to Birefringence}

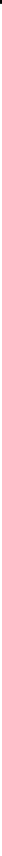

$\psi \approx \frac{N}{6}\left(\frac{g B L}{4}\right)^{2} \frac{m_{a}^{2} L}{\omega} \sin (2 \theta)$ 
K. Ehret et al. 1004.1313

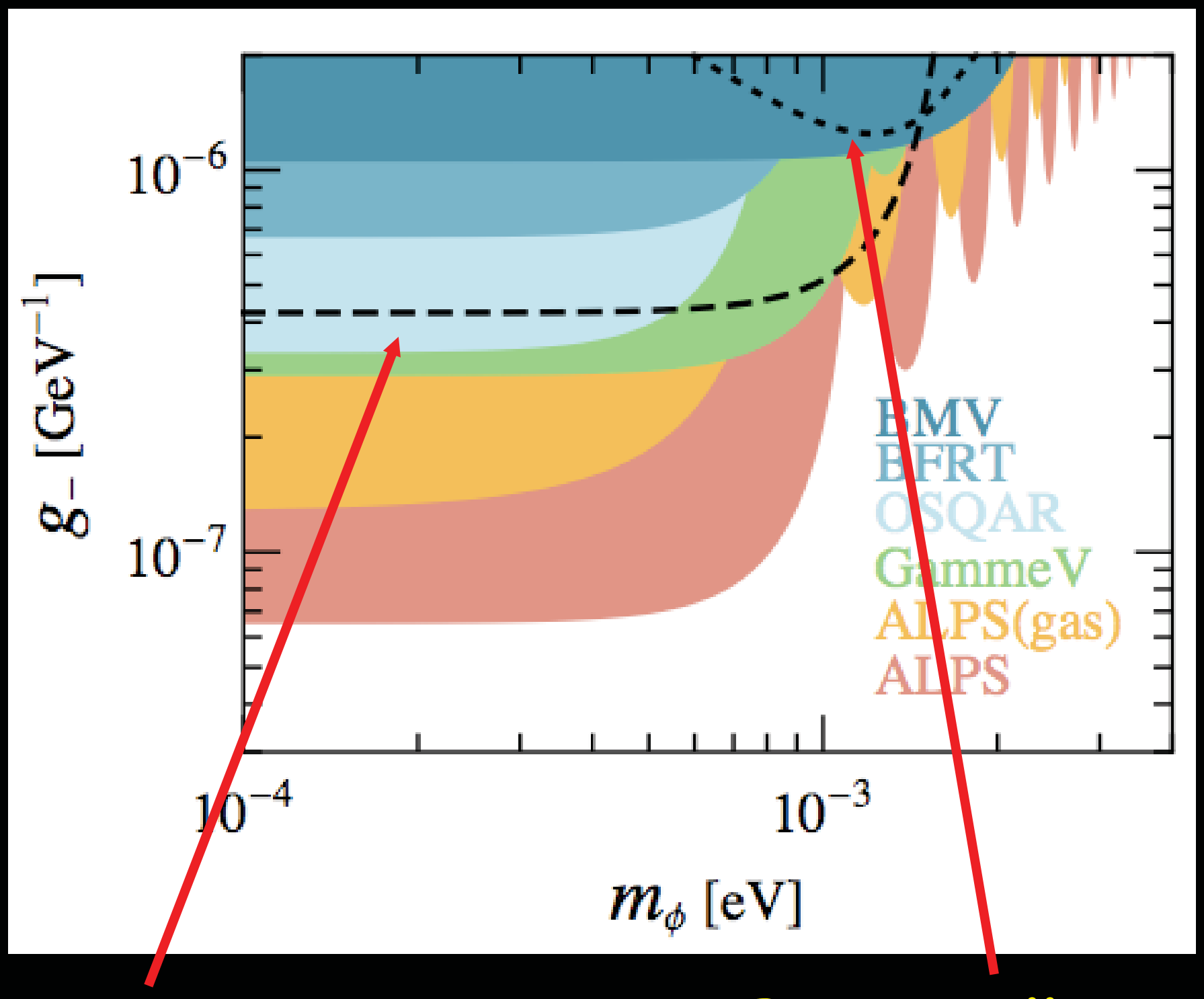

PVLAS rotation

PVLAS ellipticity 


\section{Pairproduction with a Laser}

\section{Laserphoton}

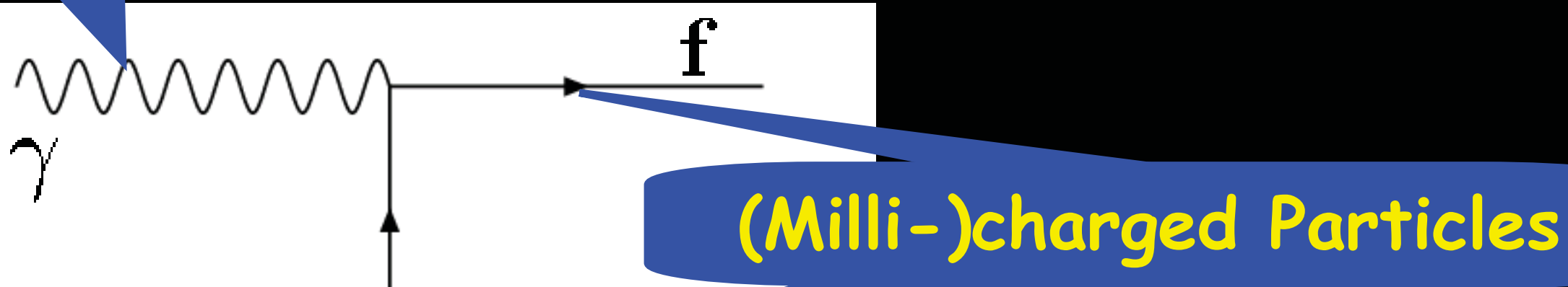

\section{External B-Feld}

\section{$\overline{\mathbf{f}}$}

$B_{\text {ext }}$ 


\section{Pairproduction with a Laser}

\section{Laserphoton}

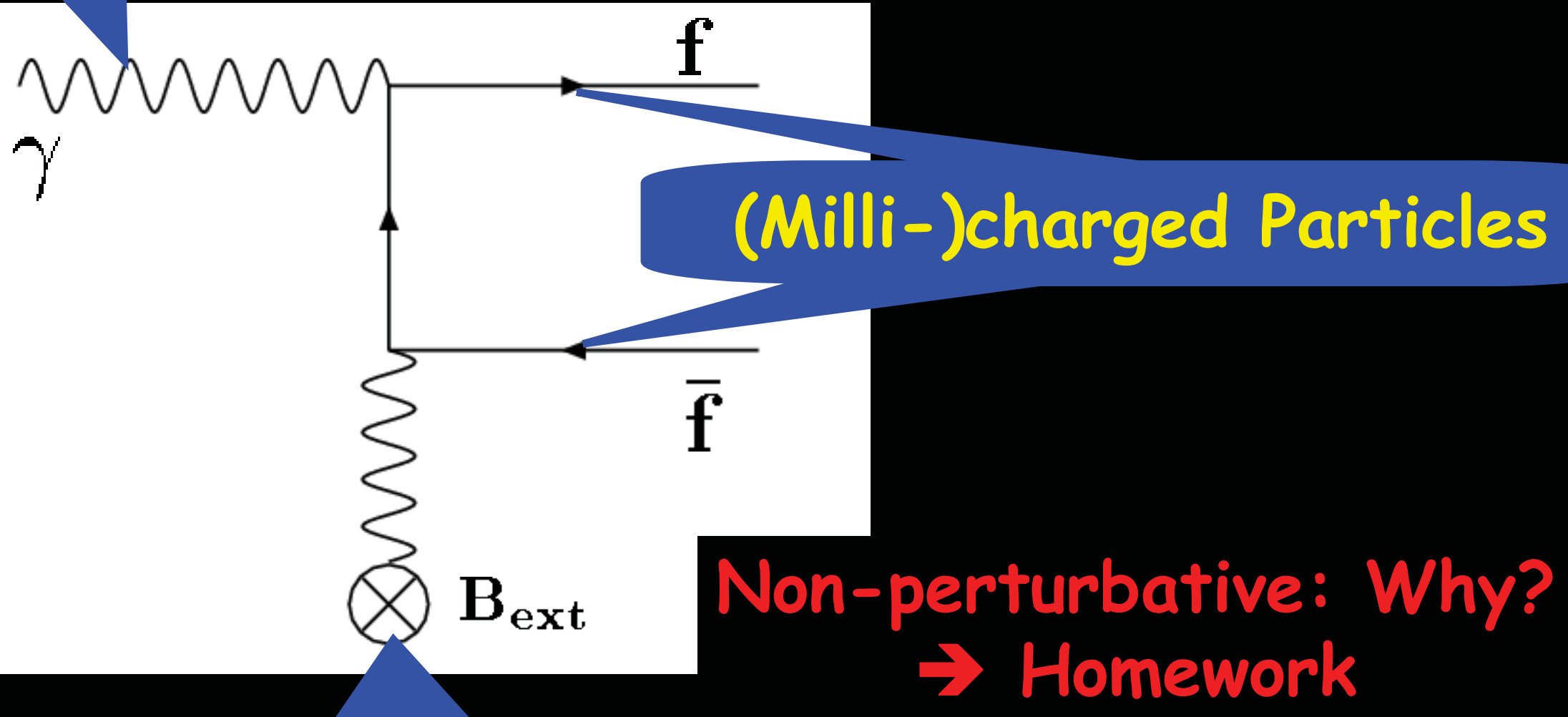

External B-Feld 


\section{Dichroism (Rotation)}

- Pairproduction leads to an "absorption" of Laser light

- Pairproduction depends on relative orientation of Laser polarization and

B-field

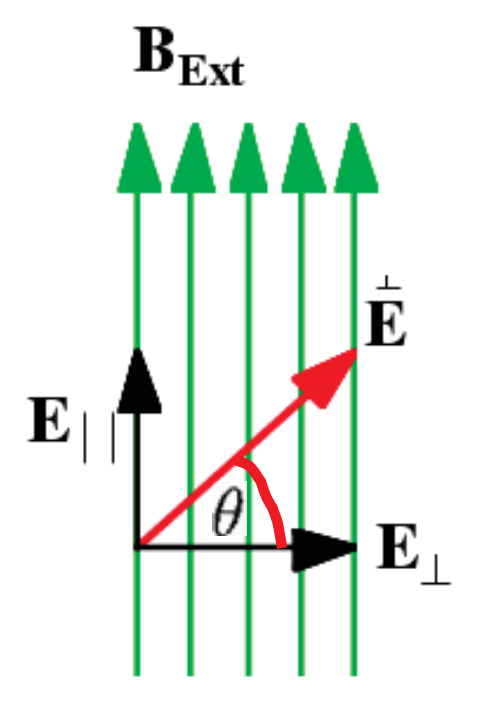

before

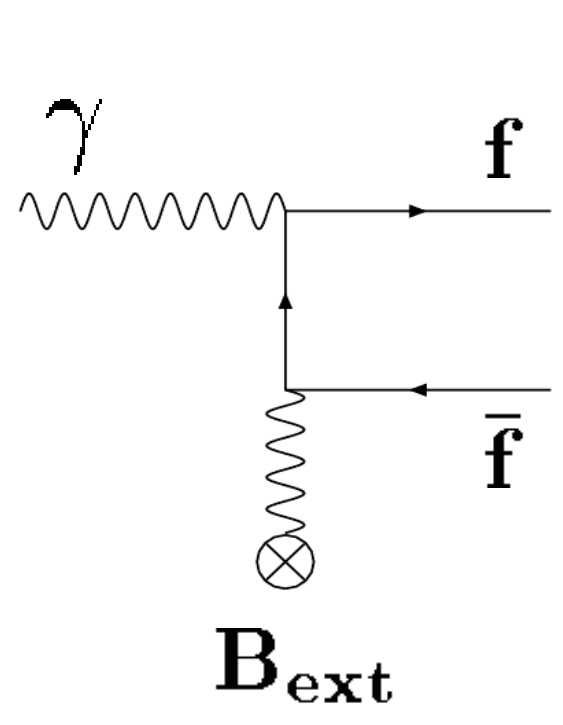

$\mathbf{B}_{\text {ext }}$

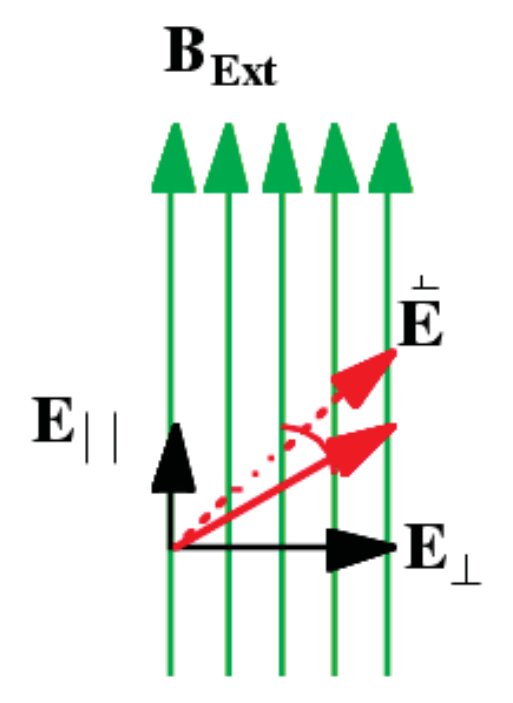

$\underline{\text { rotation } \varepsilon}$

after 


\section{Geometry}
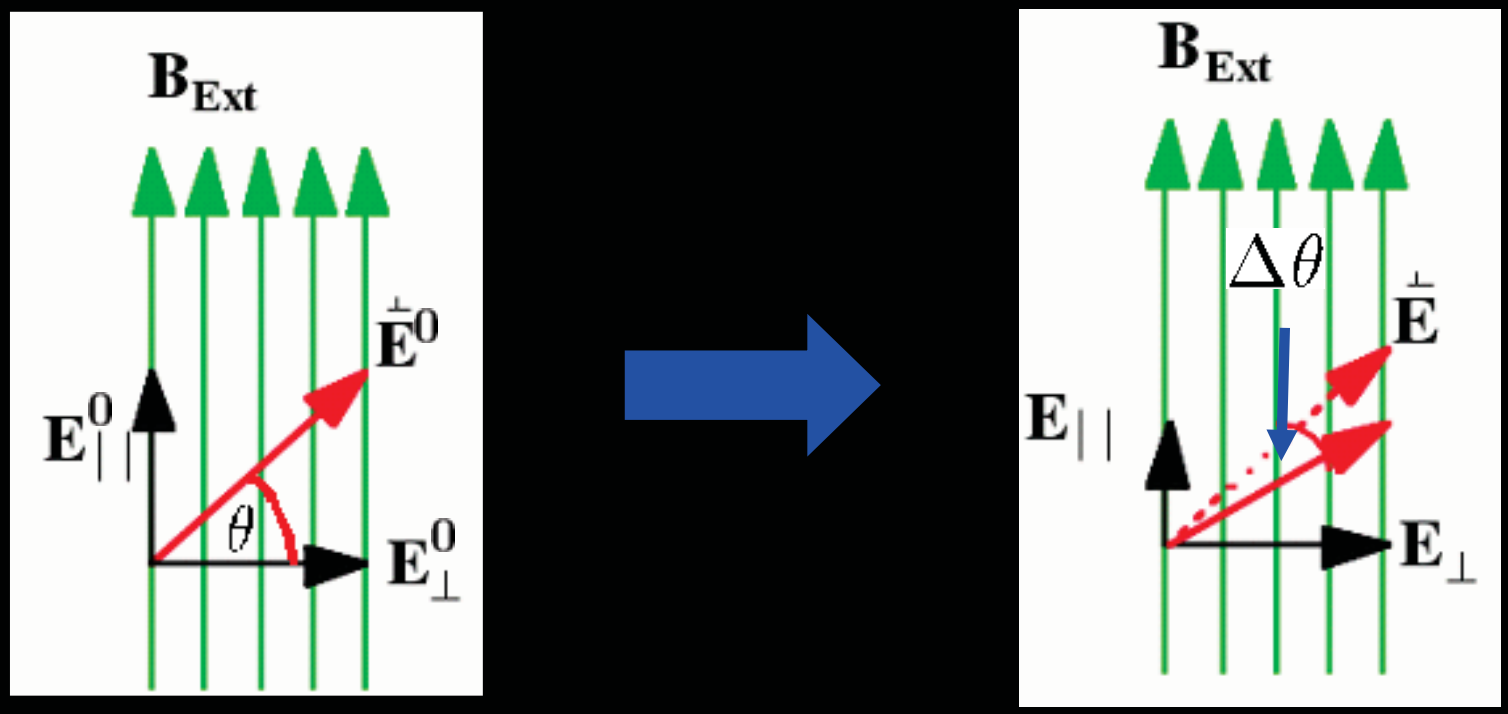

$\tan (\theta-\Delta \theta)=\frac{E_{\|}}{E_{\perp}}=\frac{E_{\|}^{0}}{E_{\perp}^{0}} \exp \left(-\frac{1}{2}\left(\kappa_{\|}-\kappa_{\perp}\right) \ell\right)$

$$
\Delta \theta \simeq \frac{1}{4}\left(\kappa_{\|}-\kappa_{\perp}\right) \ell \sin (2 \theta)
$$




\section{Minicharges}

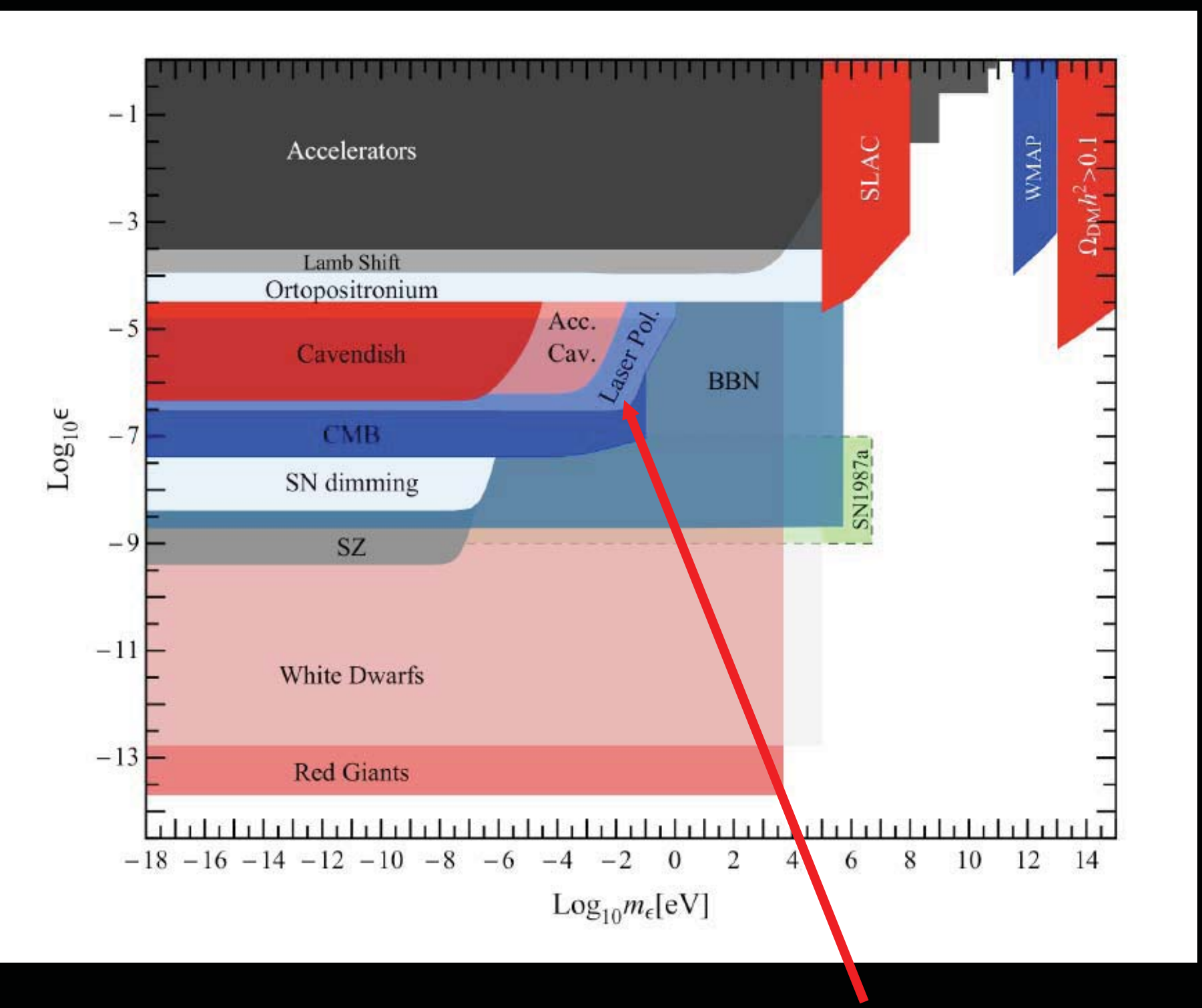

\section{One of the best laboratory bounds!}


Helioscopes 


\section{Helioscopes}

$m\left(1 P^{3} n\right.$

CAST@CERN

SUMICO@Tokyo

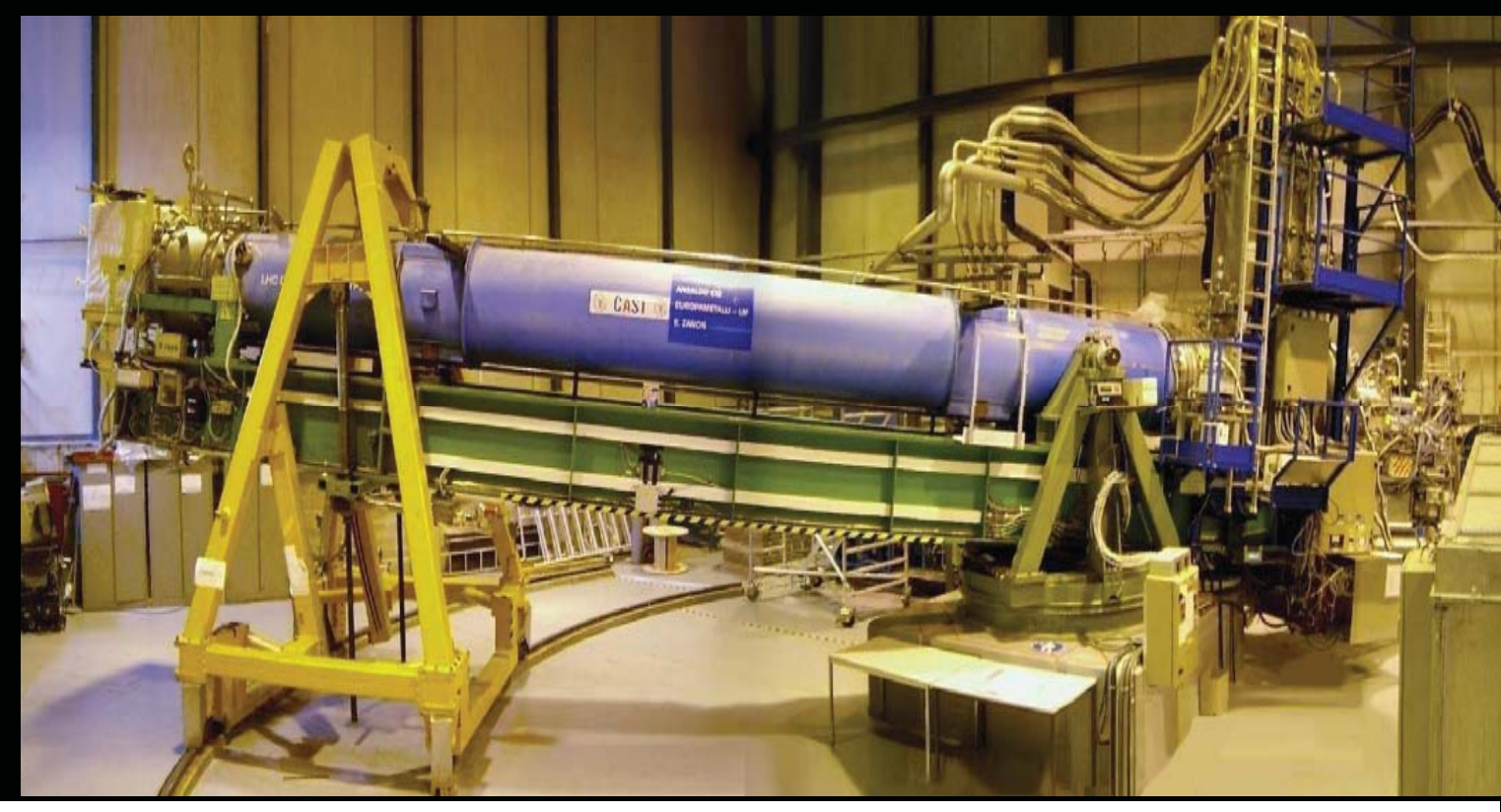

"Light shining through a wall"
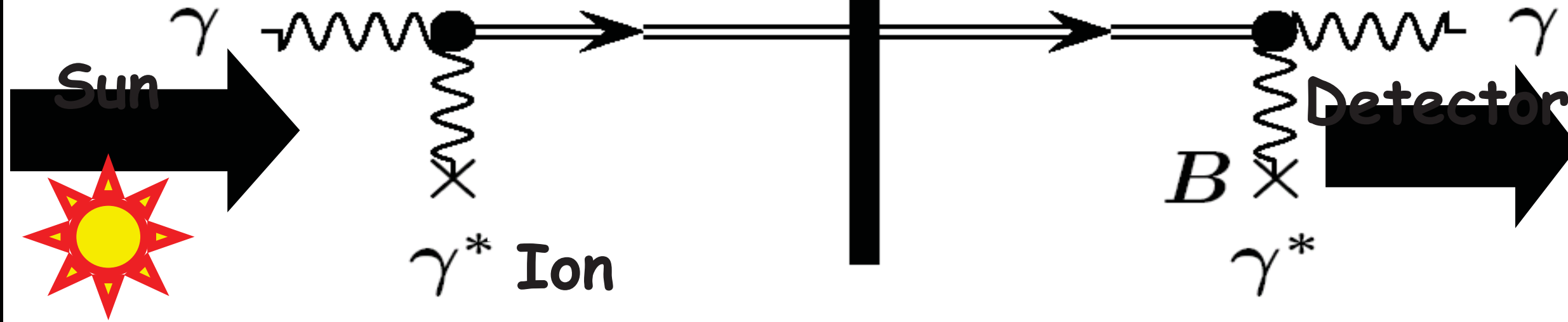

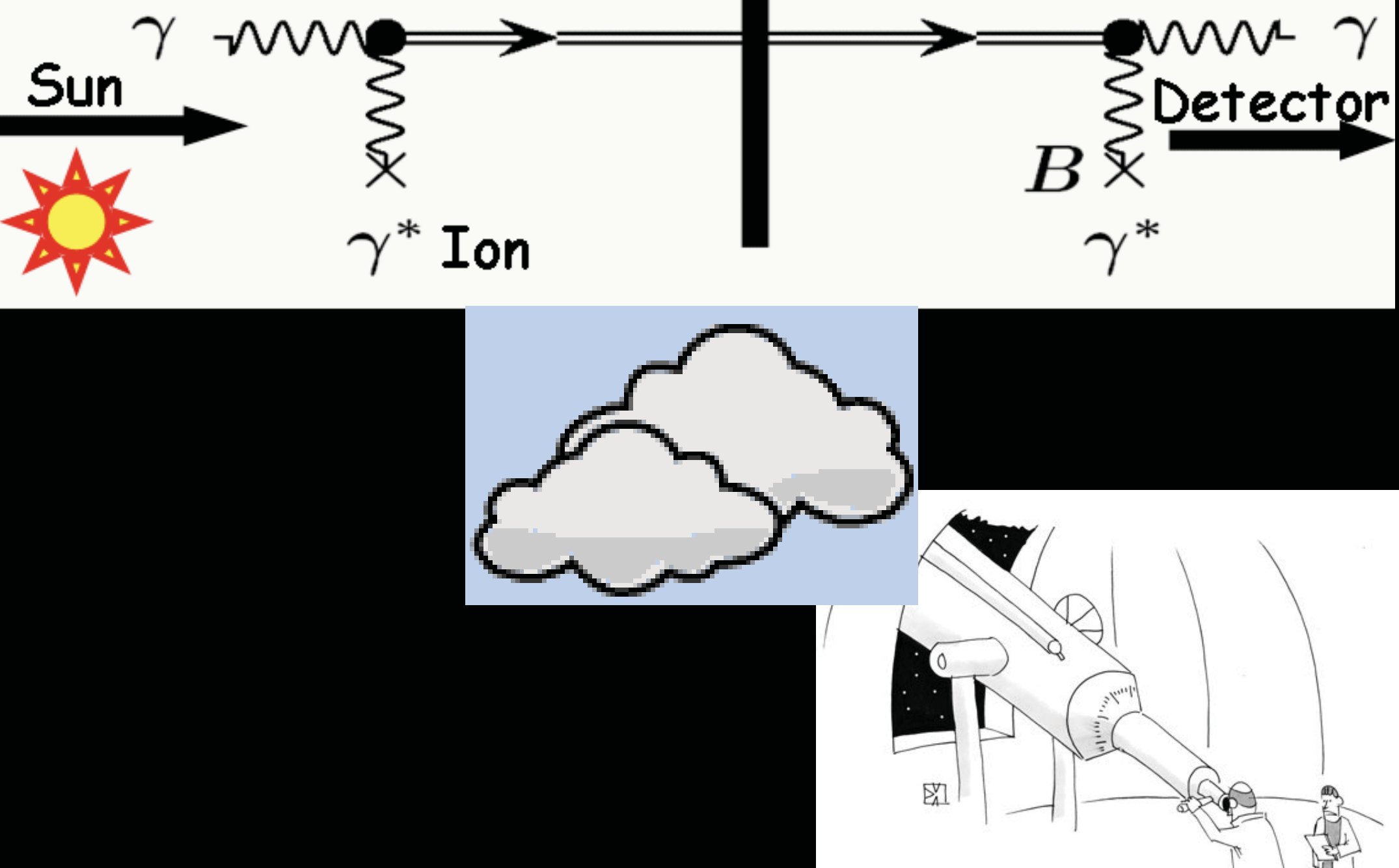

This isn't Dark matter. 


\section{Sensitivity}

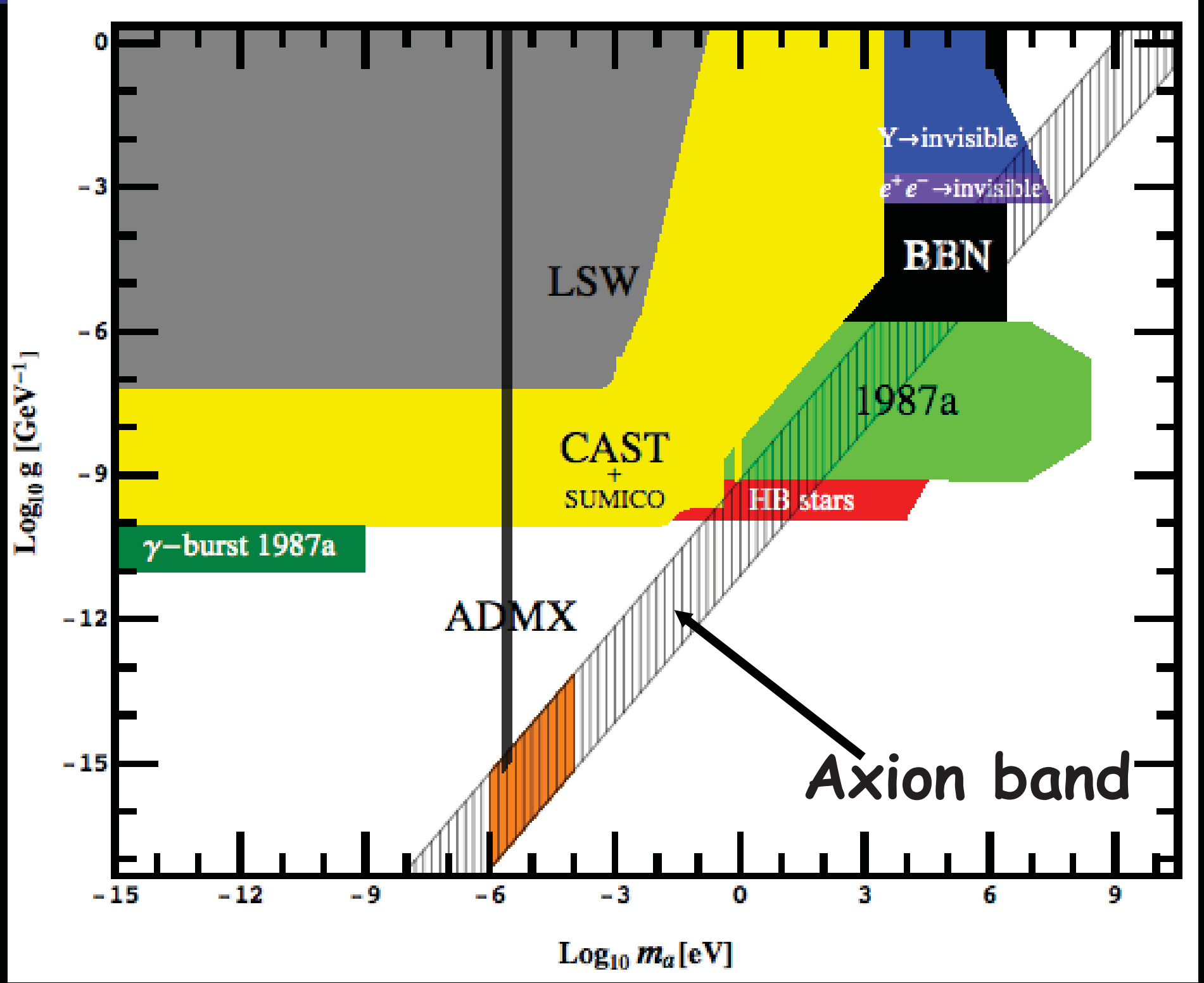




\section{Searching light Dark Matter}

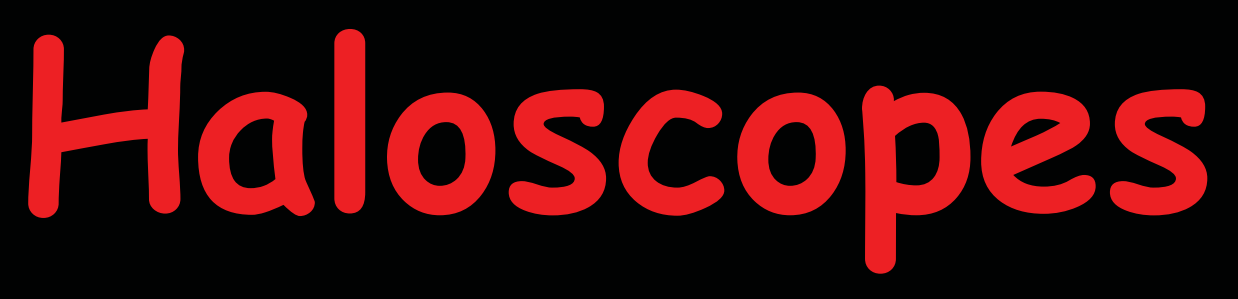

See, e.g.

G. Rybka at Patras 2009 


\section{Axions: Two photon coupling}

- Photon generation from axions!

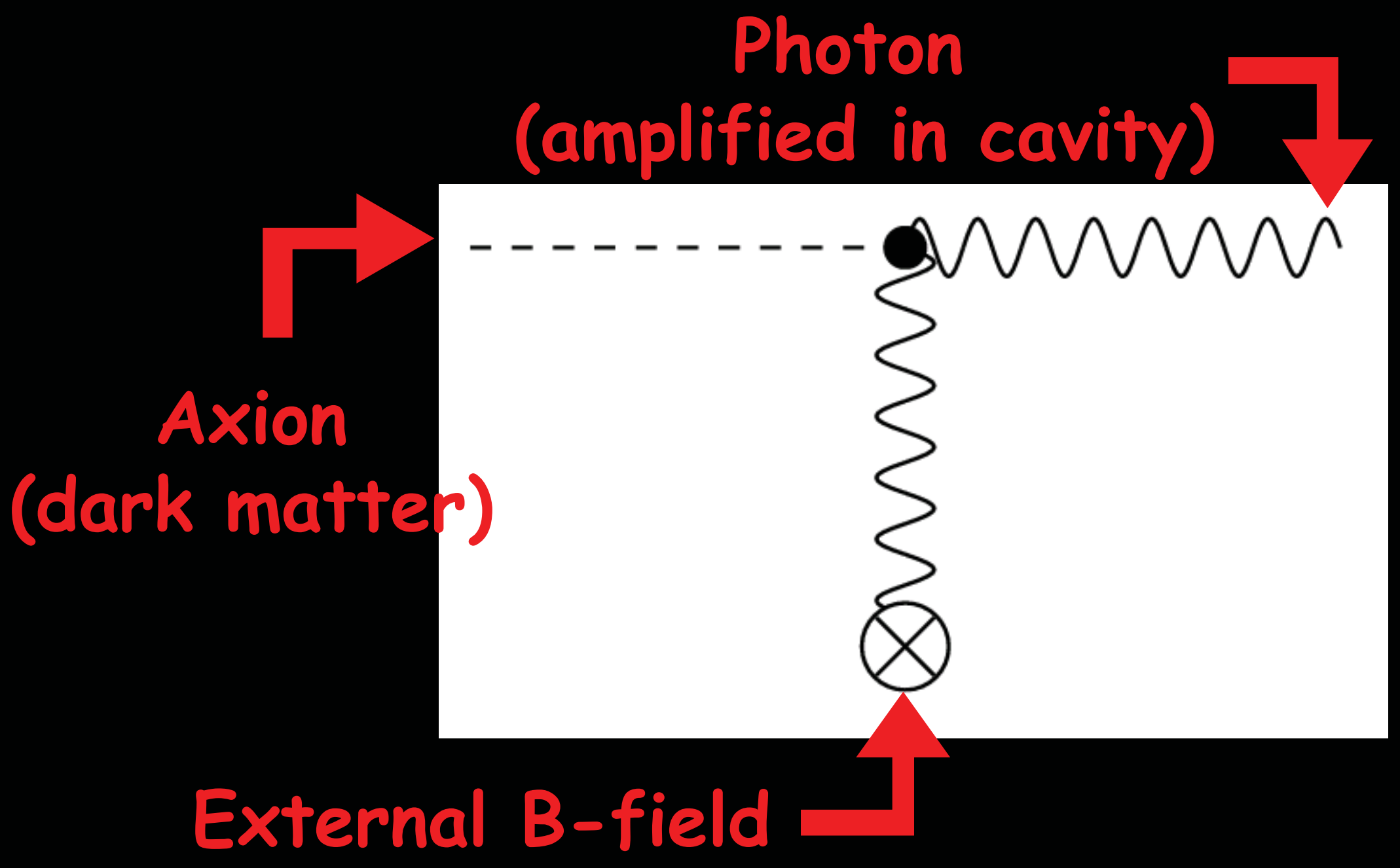




\section{Electricity from Dark Matter :-).}

$m(1 P 3) m$

- Photon Regeneration

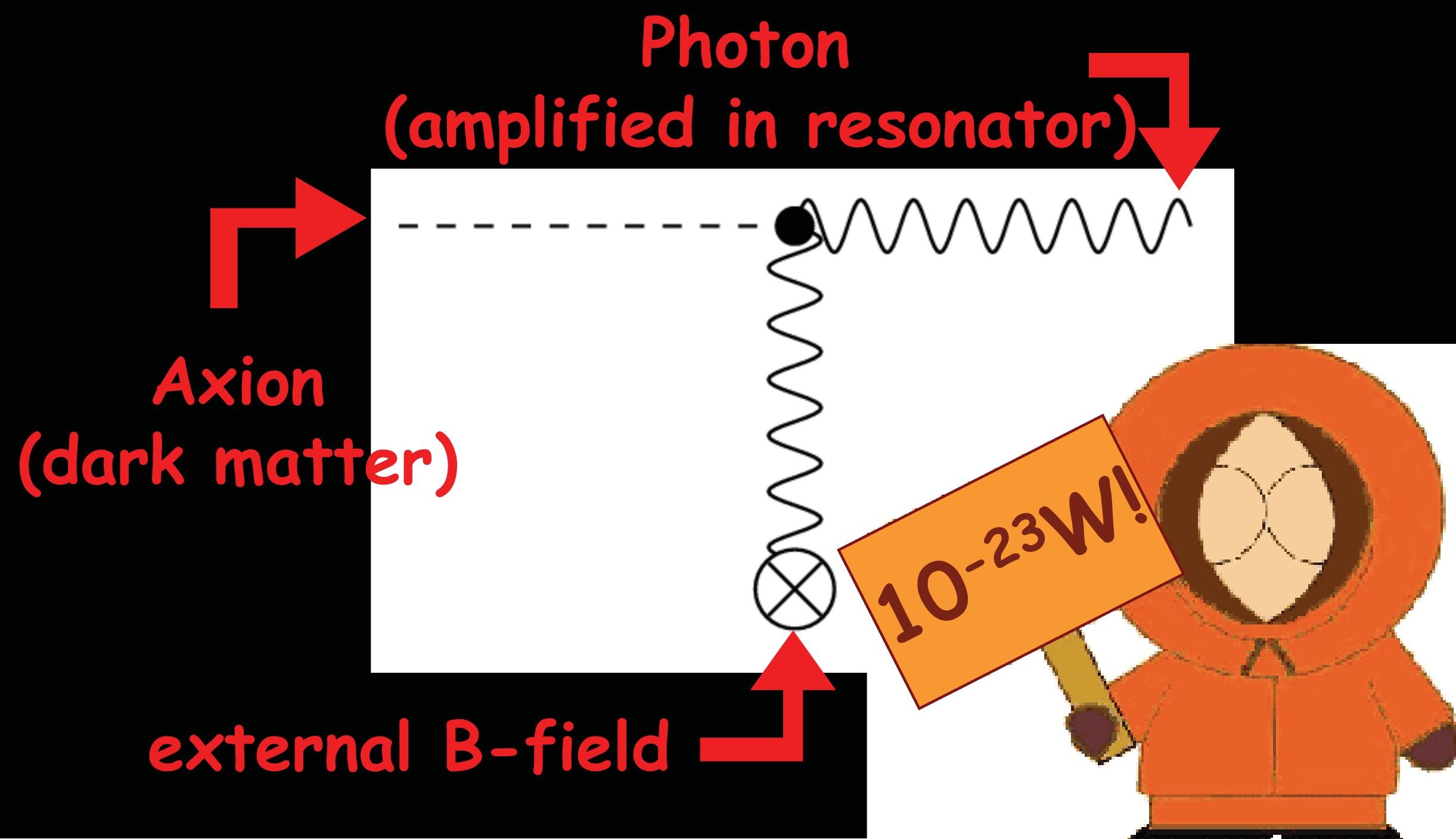




\section{Axions in Cavity}

Superconducting magnet

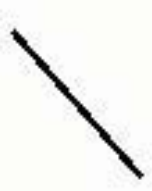

Ultra-low noise microwave receiver

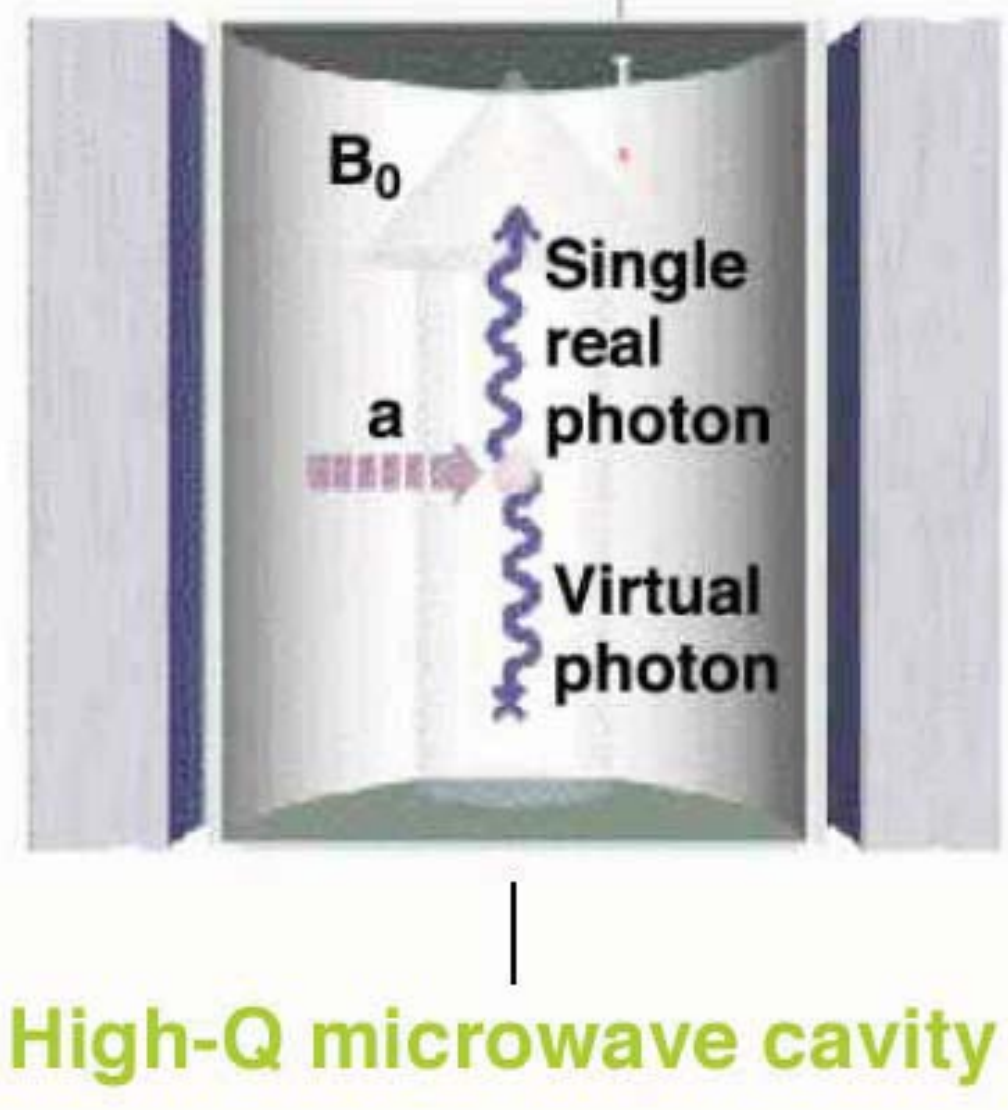




\section{Signal: Total energy of Axion}
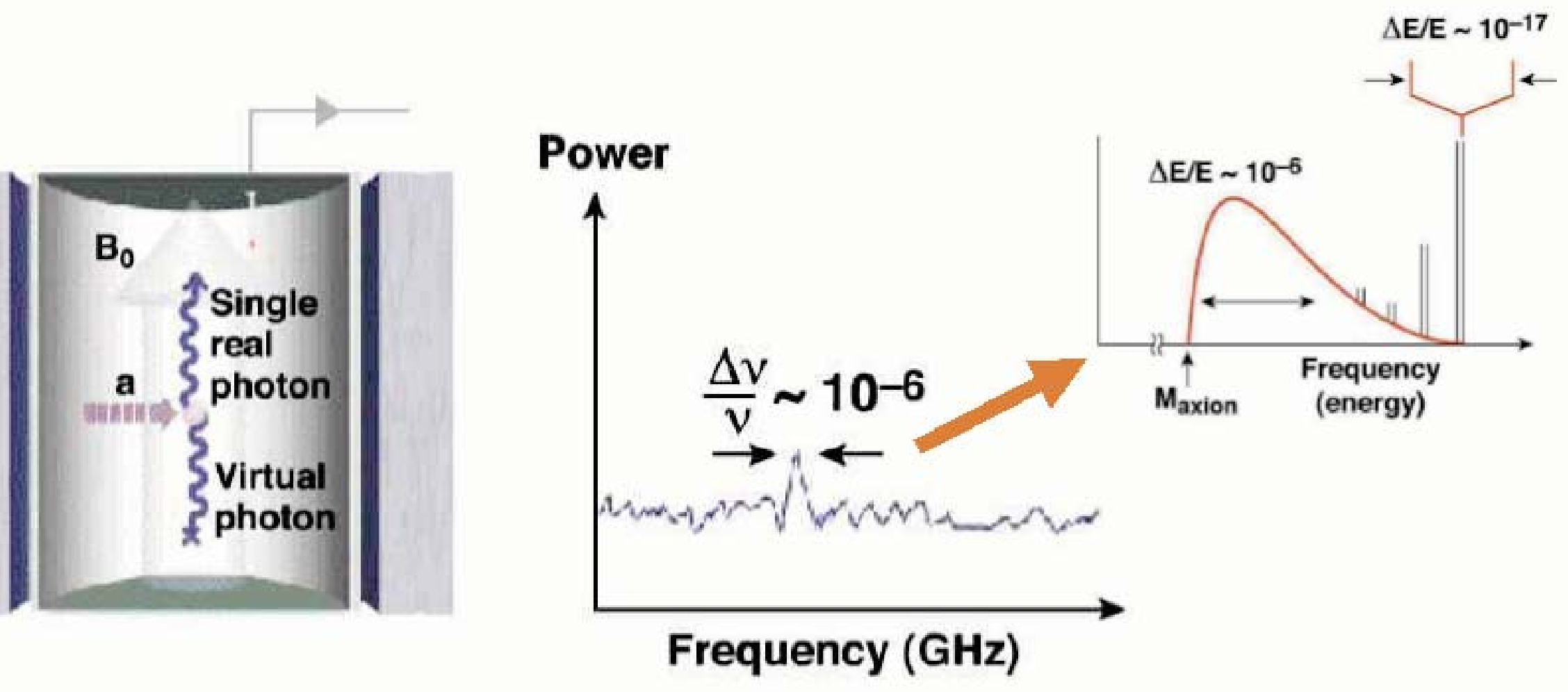

$$
\begin{aligned}
& h \nu=m_{a} c^{2}\left[1+\mathcal{O}\left(\beta^{2} \sim 10^{-6}\right)\right] \\
& \text { Virial velocity }
\end{aligned}
$$




\section{Optimizing}

$P \sim 10^{-22} W \frac{\mathrm{Vol}}{10 l}\left(\frac{B}{6 T}\right)^{2} \min \left(1, Q \frac{\Delta \nu}{\nu}\right)$

$$
s / n=\frac{\text { signal }}{\text { noise }} \sim \frac{P}{k T} \sqrt{\frac{t}{\Delta \nu}}
$$




\section{How it looks I}

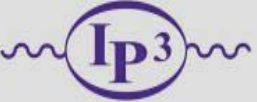

Magnet with Insert (side view)

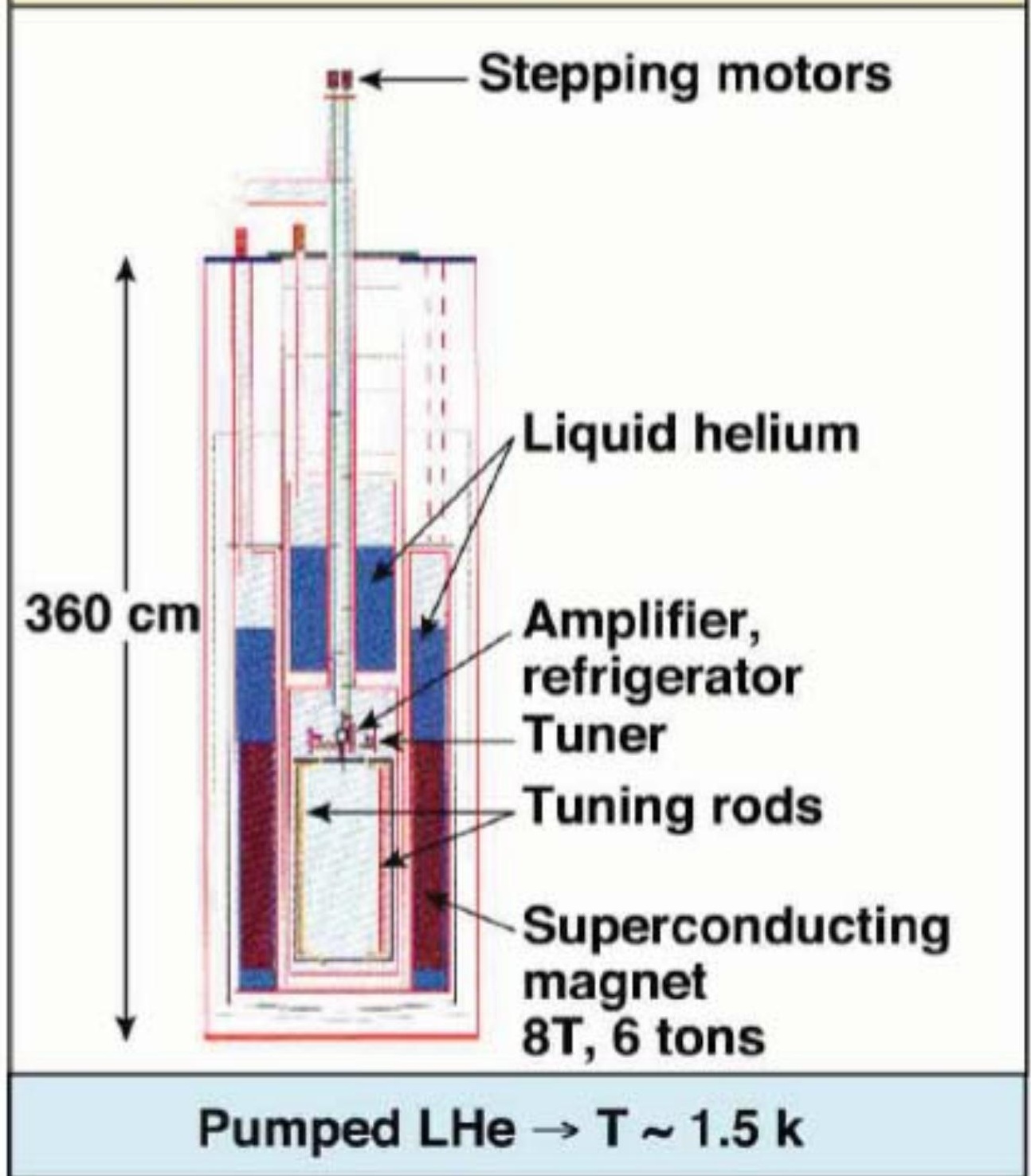

\section{Magnet (Wang NMR Inc.)}

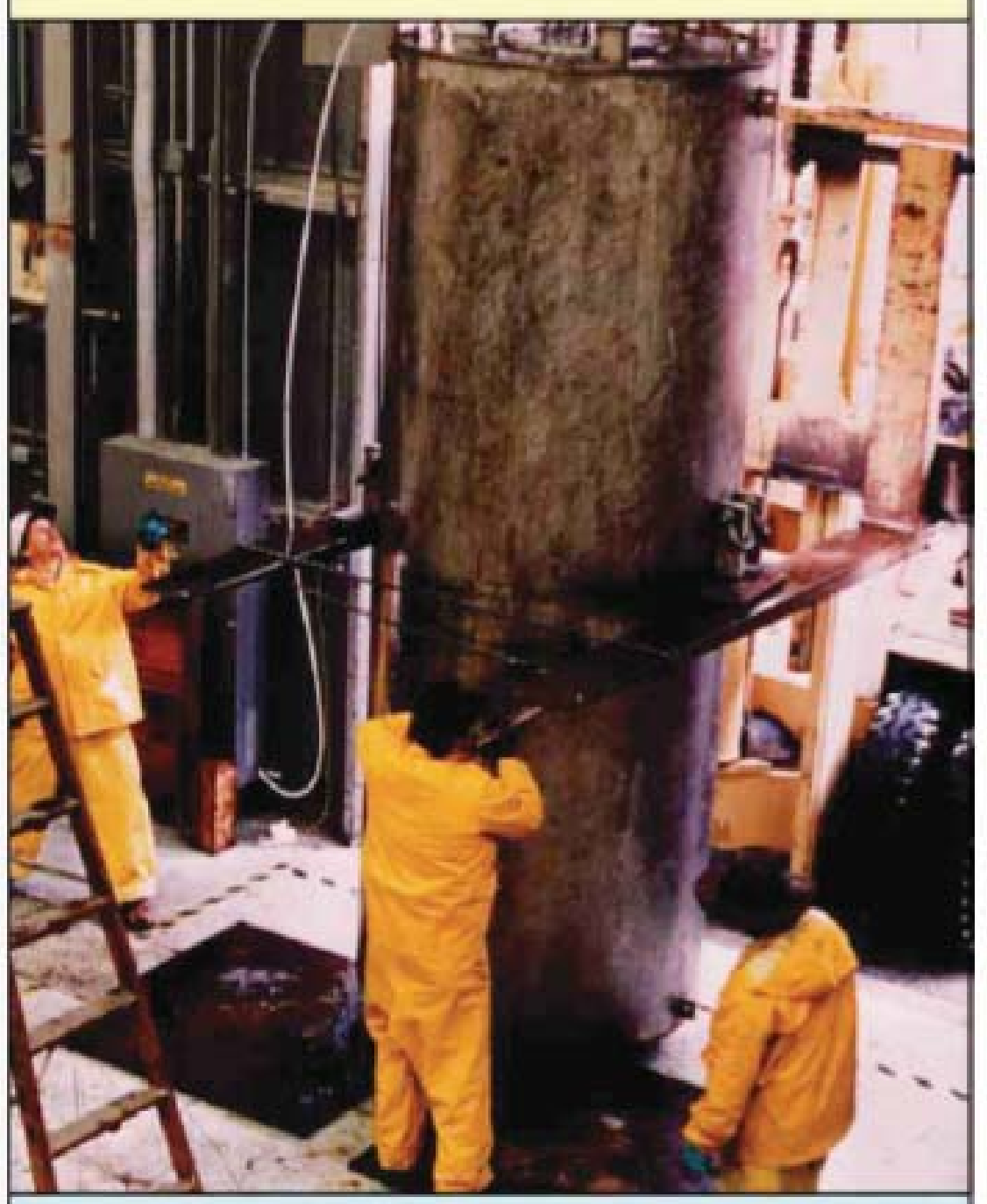

$8 \mathrm{~T}, 1 \mathrm{~m} \times 60 \mathrm{~cm} \varnothing$ 


\section{How it looks II}

$m(1 / P 3 m$

High-Q Cavity $(\sim 200,000)$

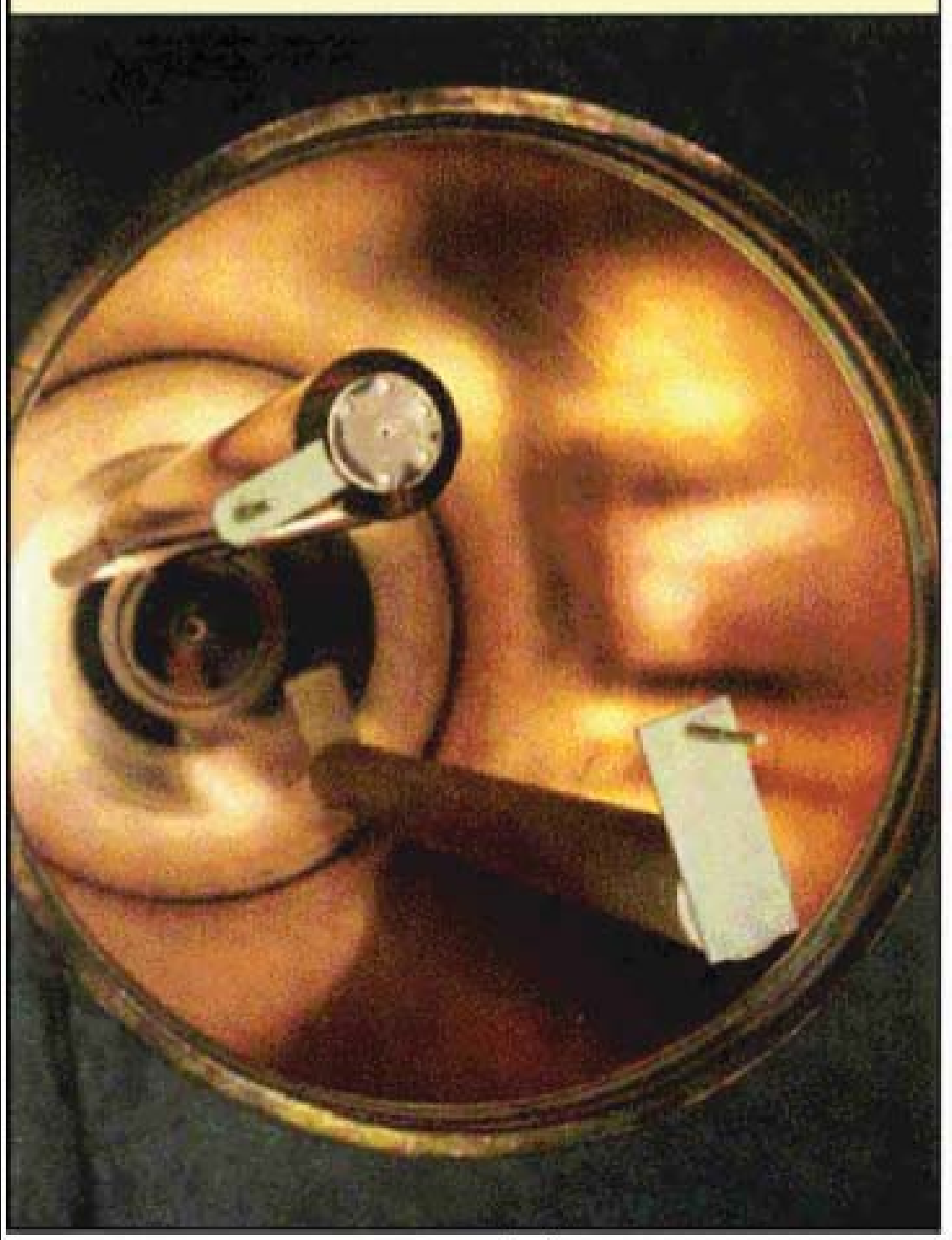

\section{Experimental Insert}

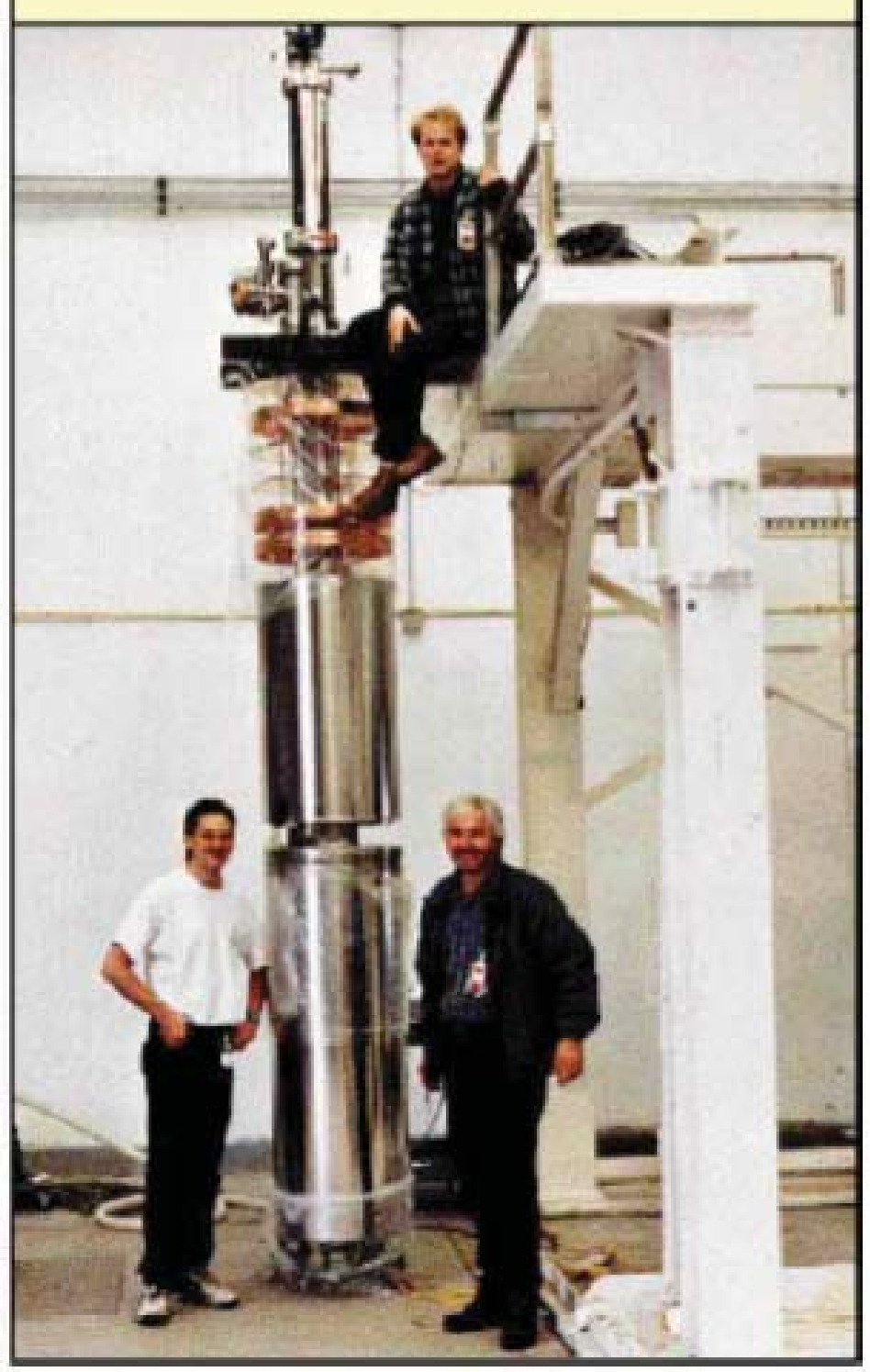




\section{Results}

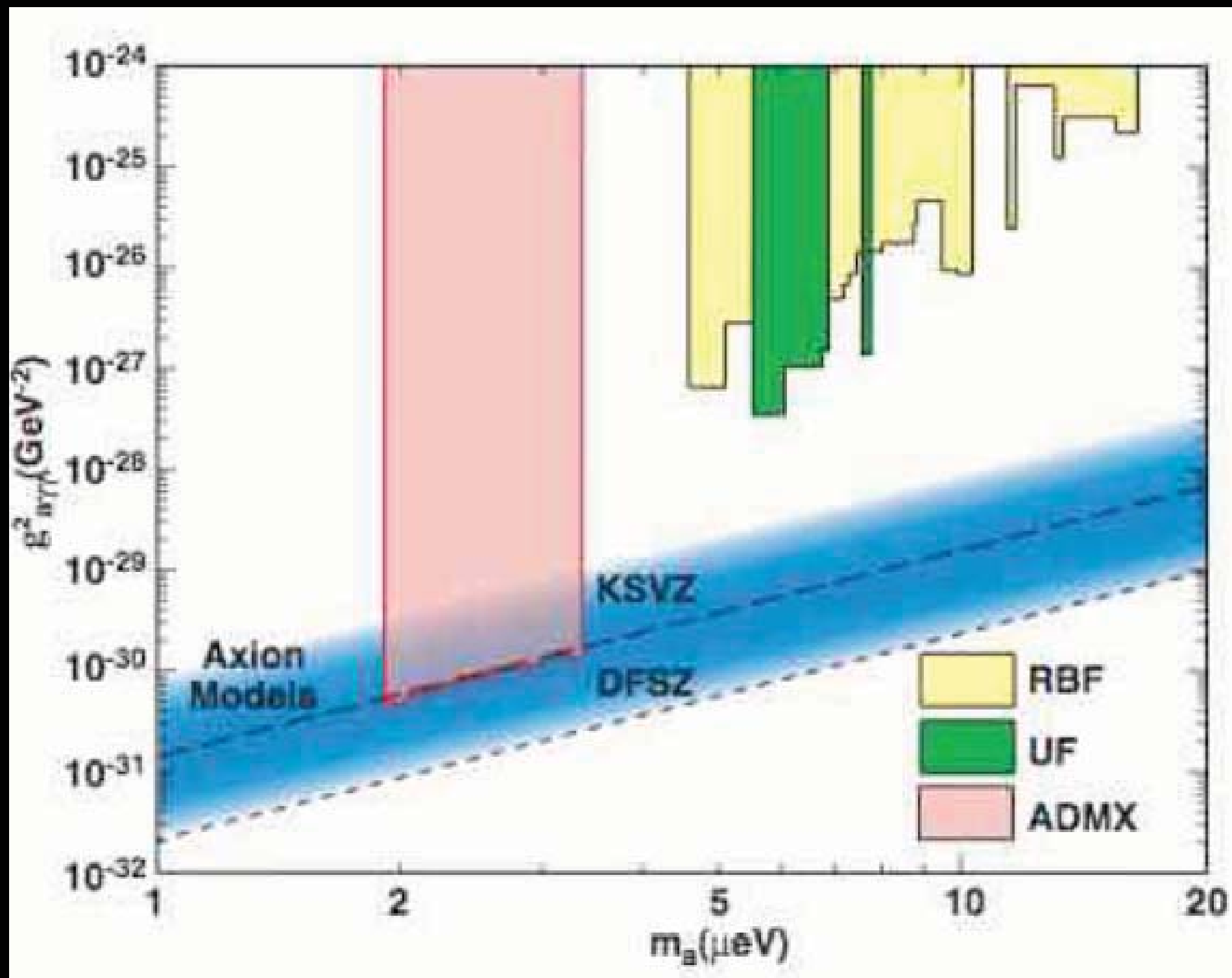




\section{Exciting times!!!}

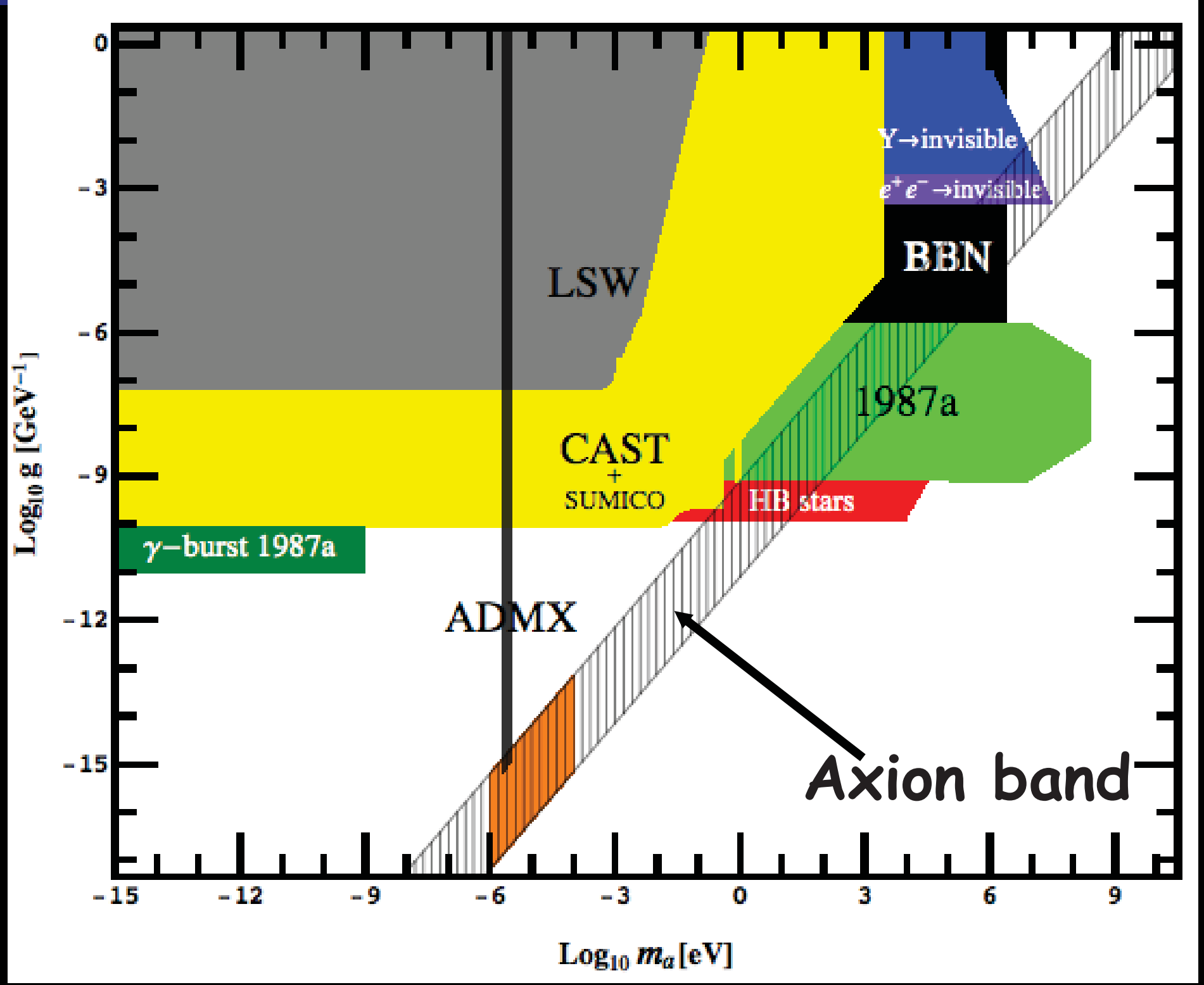




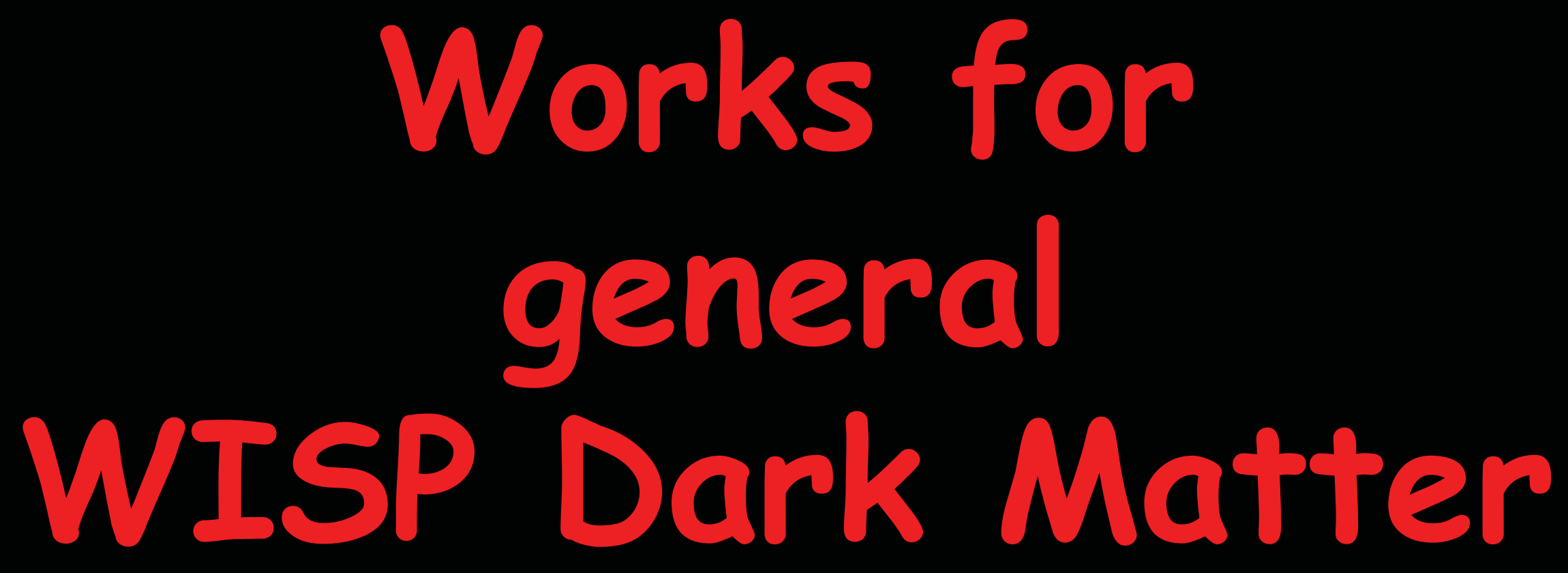

Nelson and J. Scholtz 1105.2812

P. Arias, D. Cadamuro, M.Goodsell, JJ, J. Redondo A. Ringwald 1201.5902 


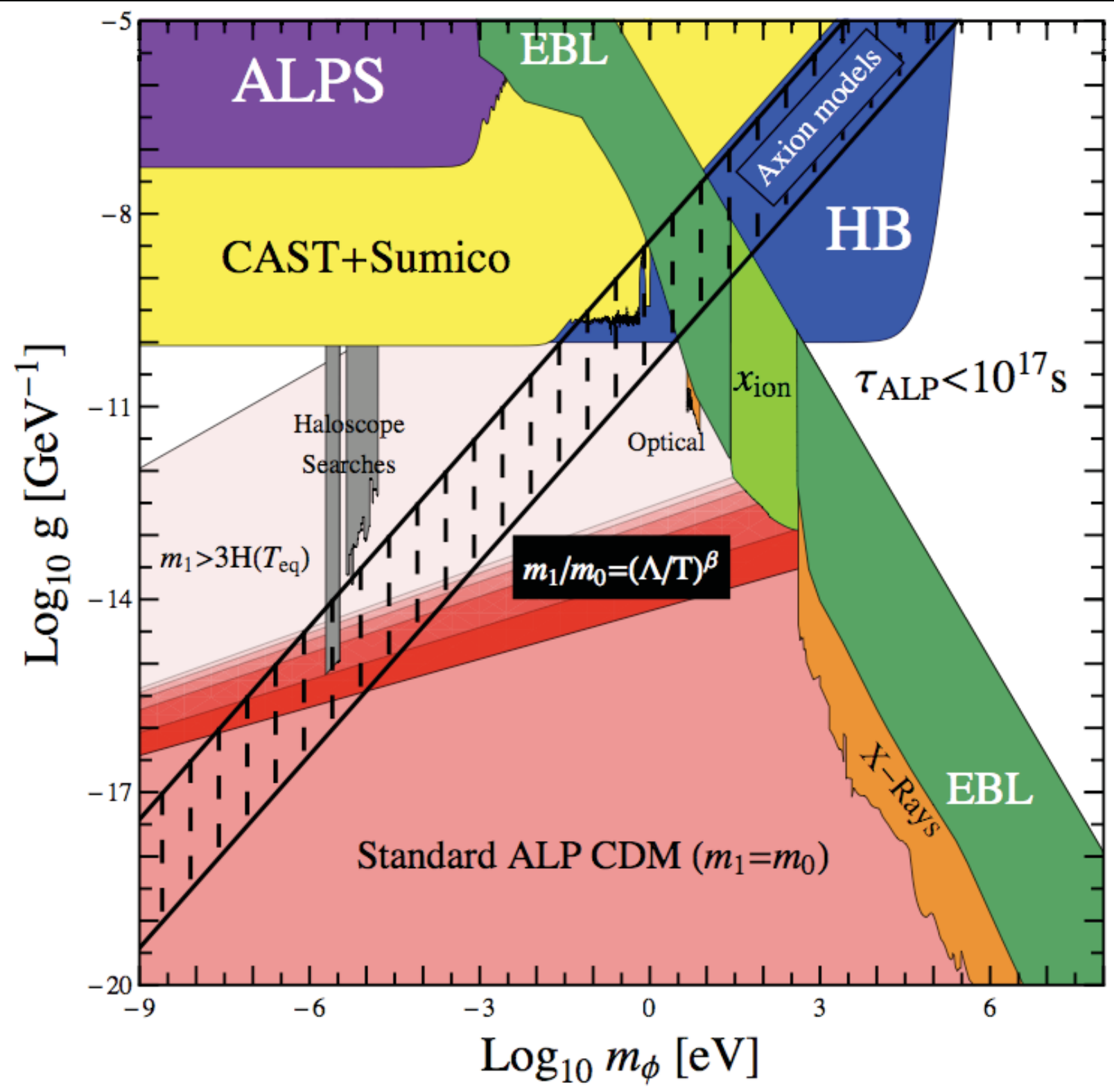




\section{Hidden Photons}

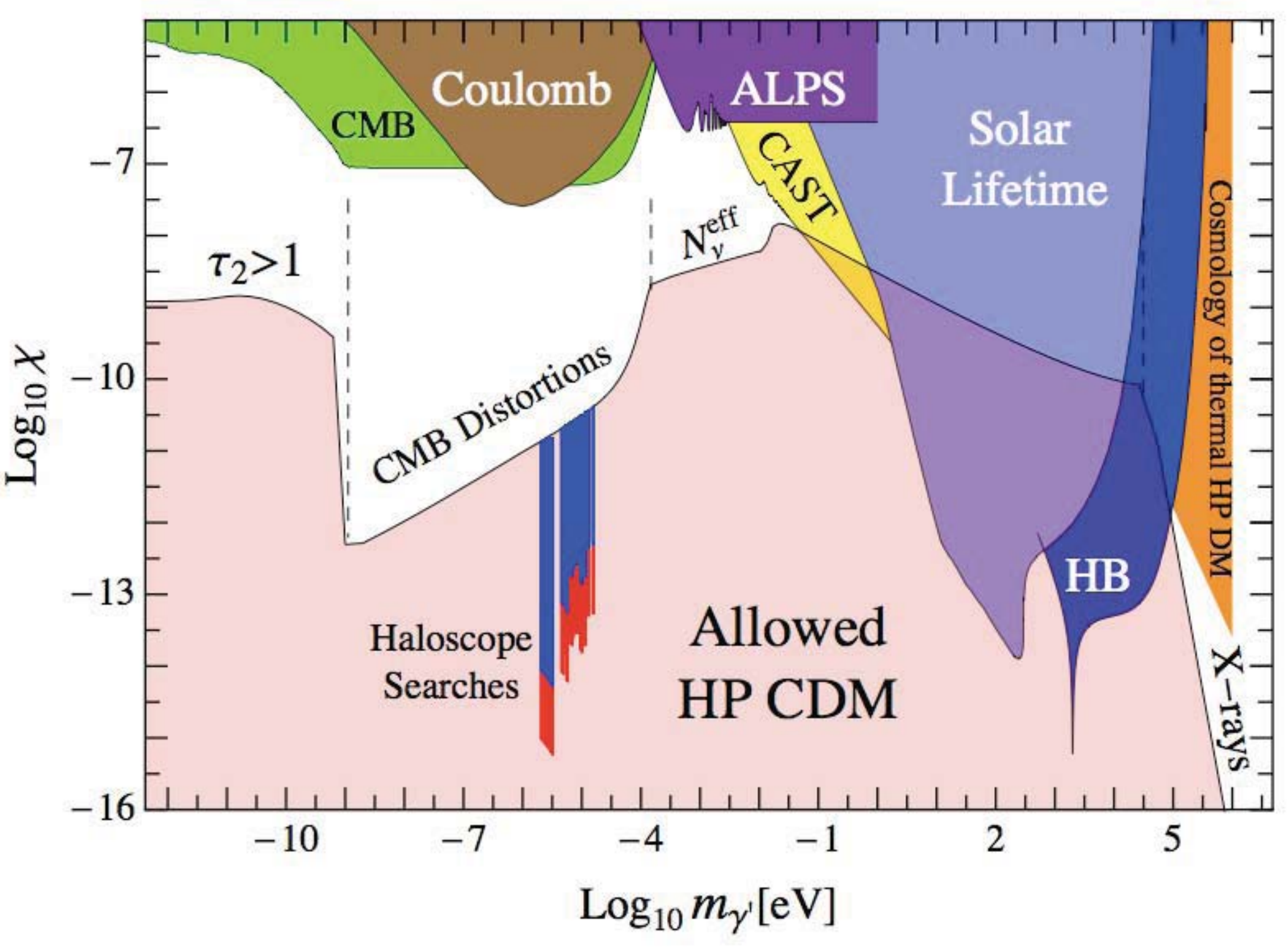




\section{Measuring}

structure (formation)

See, e.g.

S. Asztalos at Patras 2007 


\section{Example: infall of matter}

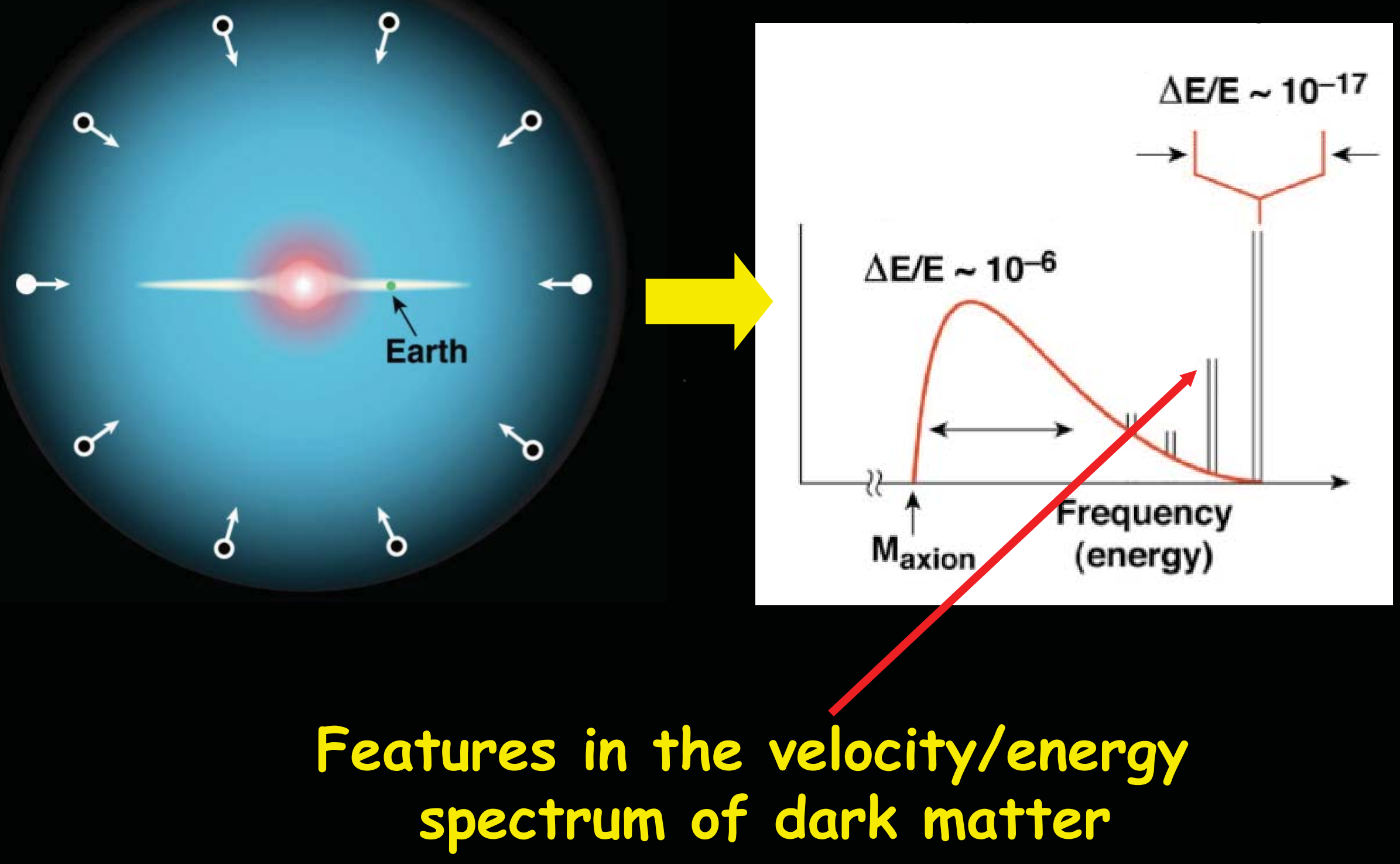




\section{Detect these features in ADMX}

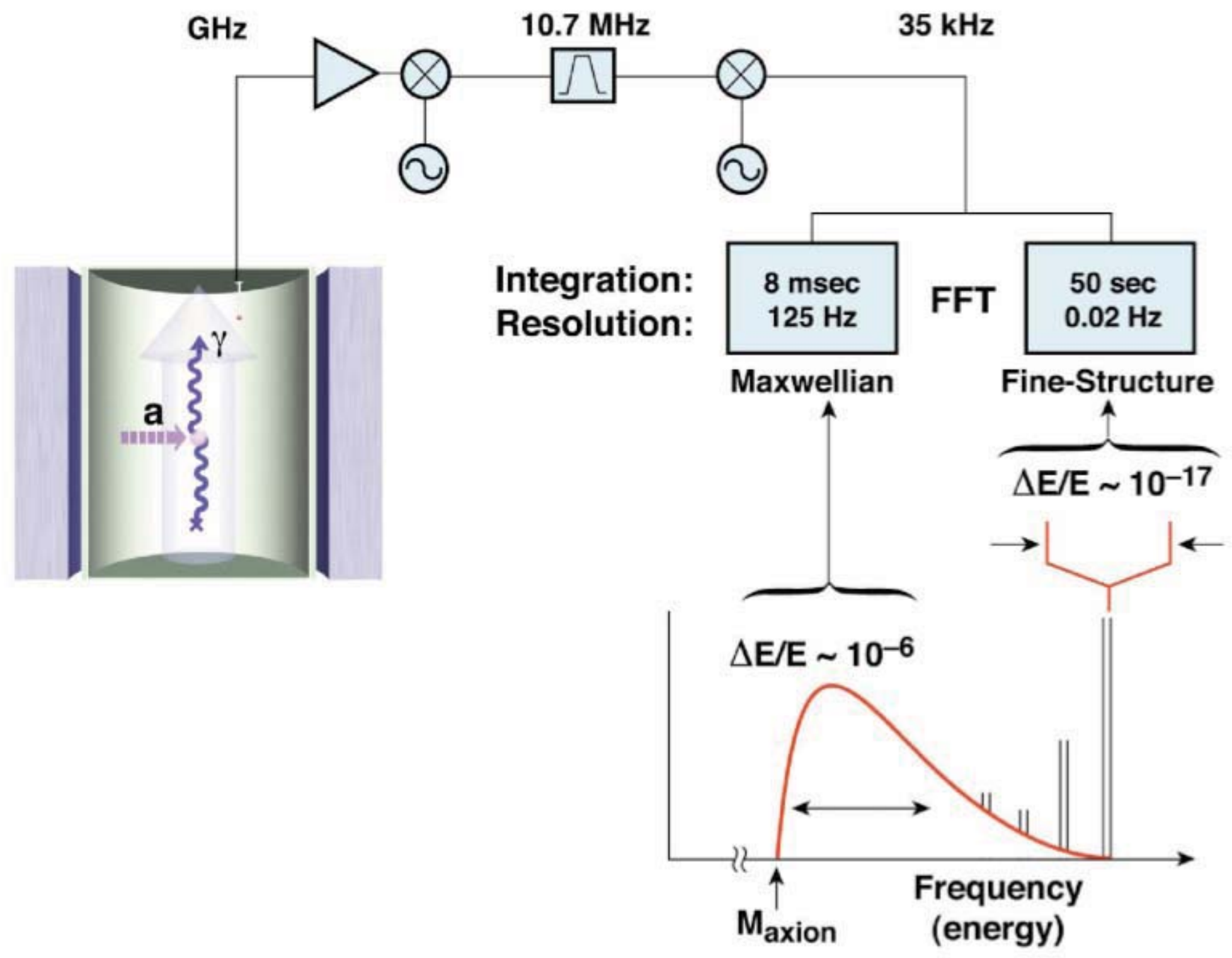




\section{Searching WISPishs*}

"Weakly interacting sub-TeV particles 


\section{Why?}

- Lots of interest from astrophysics

- Pamela, DAMA, CoGent...

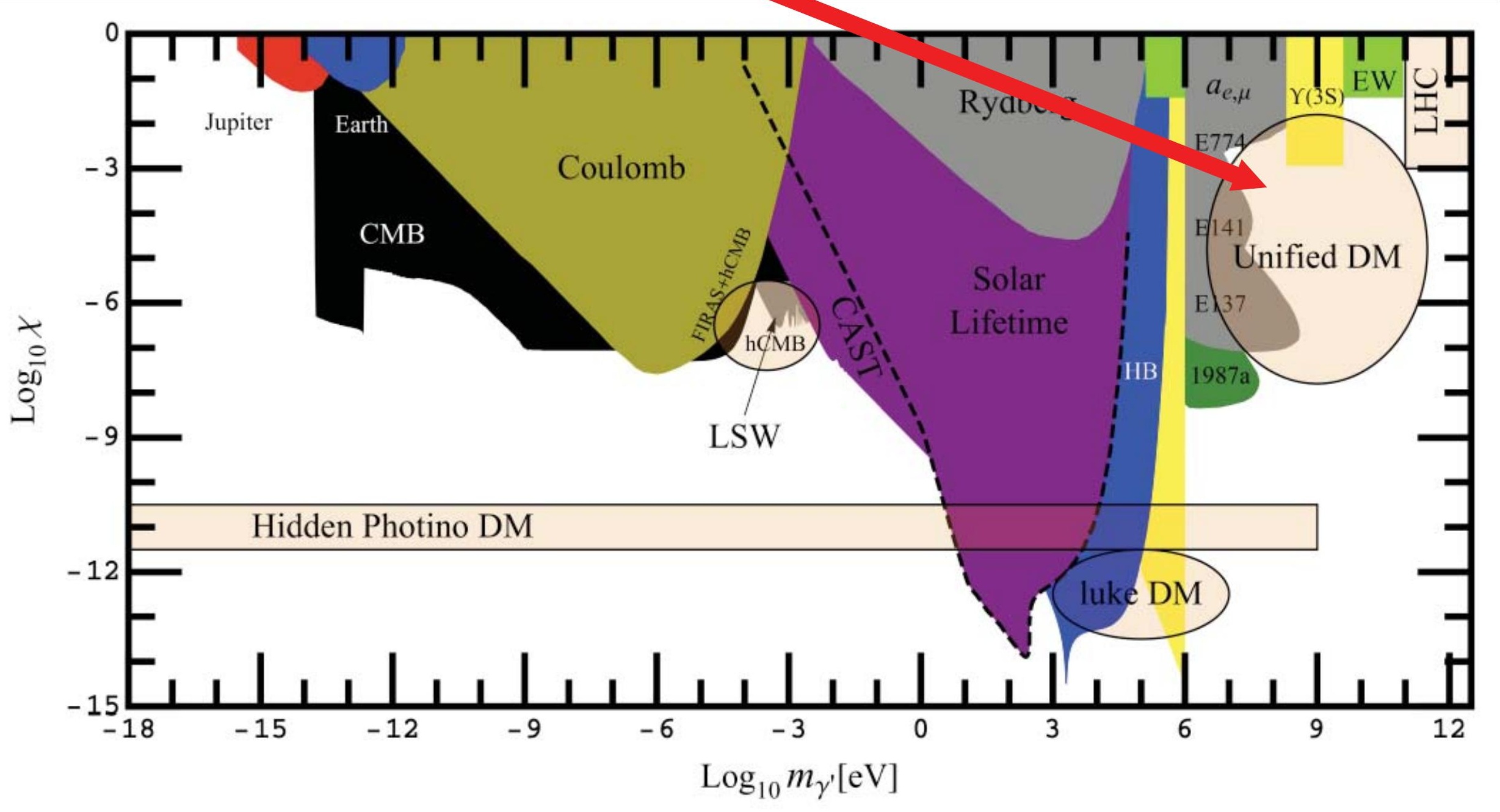




\section{and $(g-2)_{\mu}$}

- 2.x $\sigma$ deviation from SM observed

- Hidden photon could account for this!
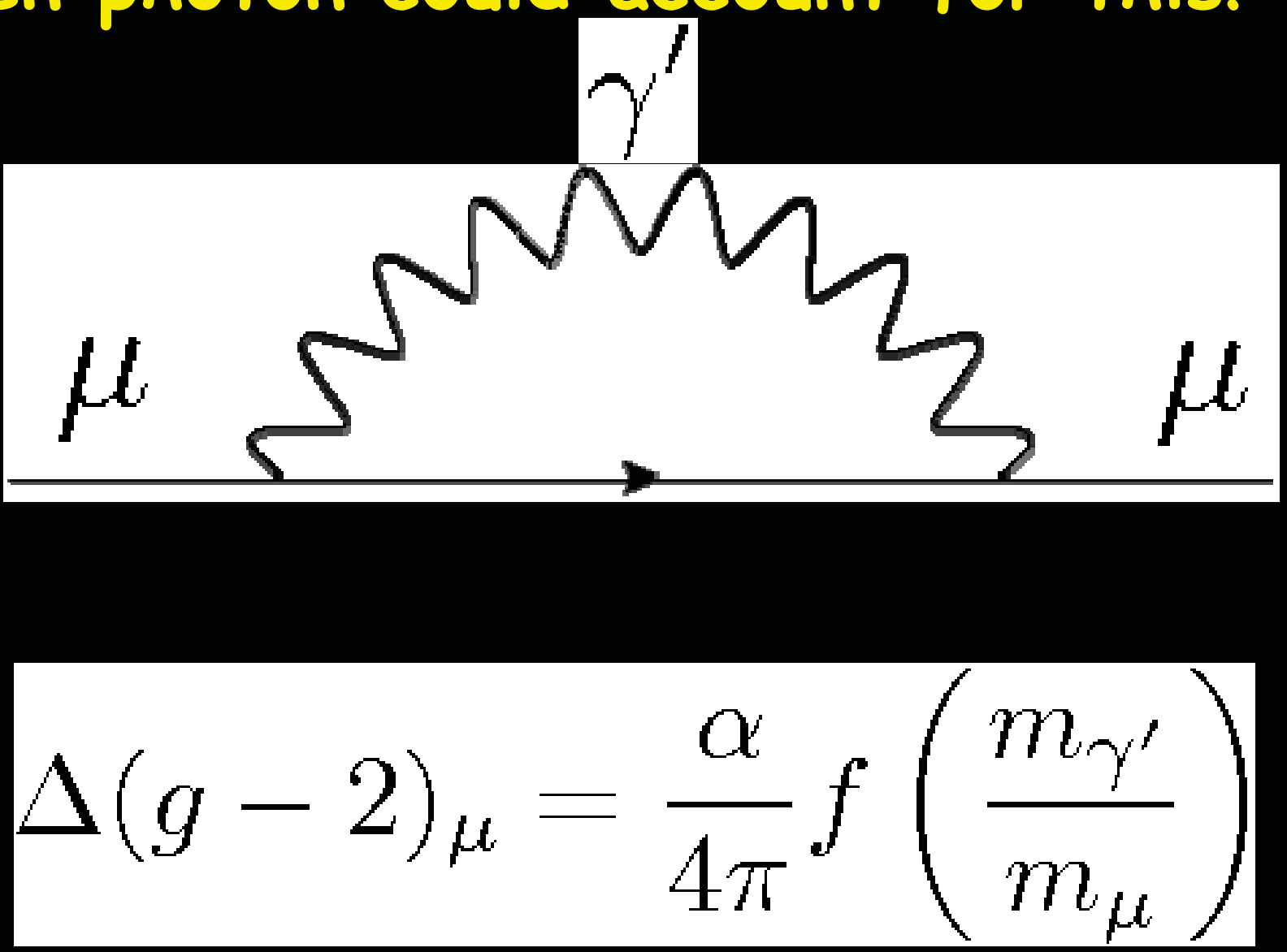


\section{Fixed targets}

Displaced Vertex

$$
\sim\left(\frac{m_{\gamma^{\prime}}}{E}\right)^{3 / 2} \quad \text { (narrow) }
$$

$e^{-}$ ANANMNAN
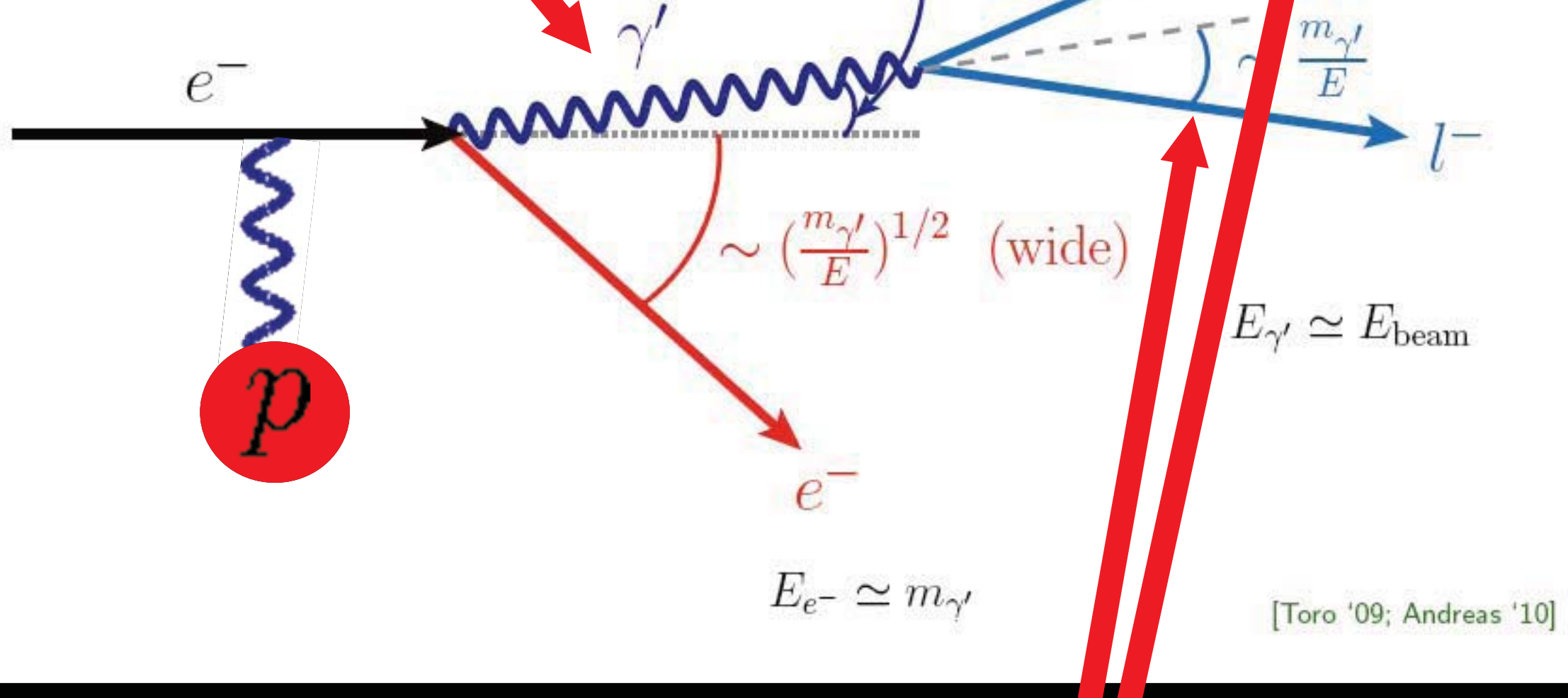

\section{Detector}


- Medium energies of beam $100 \mathrm{MeV}-50 \mathrm{GeV}$

- BUT: High current $\sim m A \sim 10^{16} e^{-/ s}$

Test weak interactions!! 
Many beams, many chances...

- DESY

- Jefferson Lab

- Mainz Microtron (MaMi) 


\section{Current state}

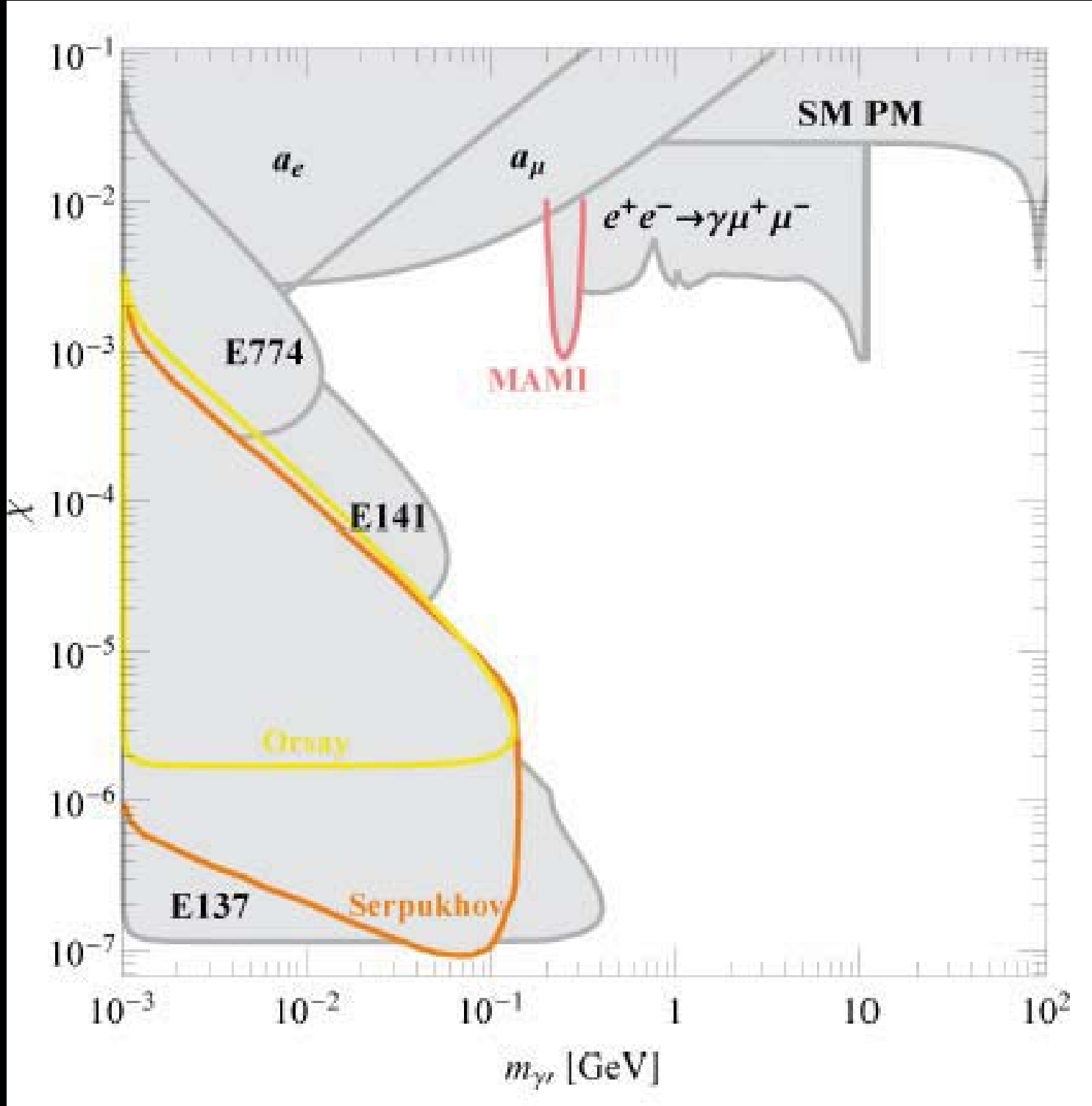

Cf. S. Andreas, M. Goodsell, A. Ringwald 1109.2869 


\section{Near future}

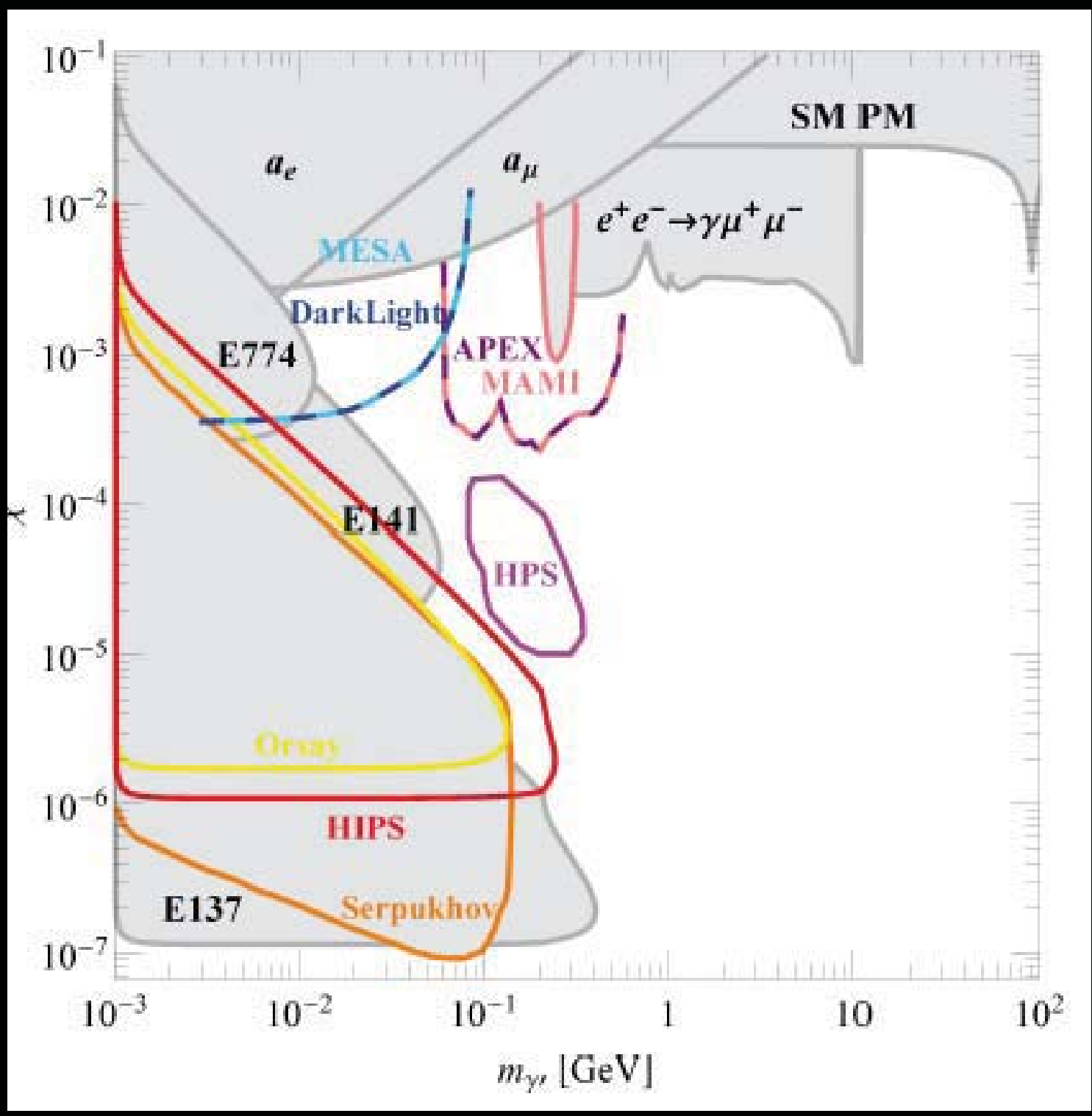




\section{Even More Hints...}

Muonic

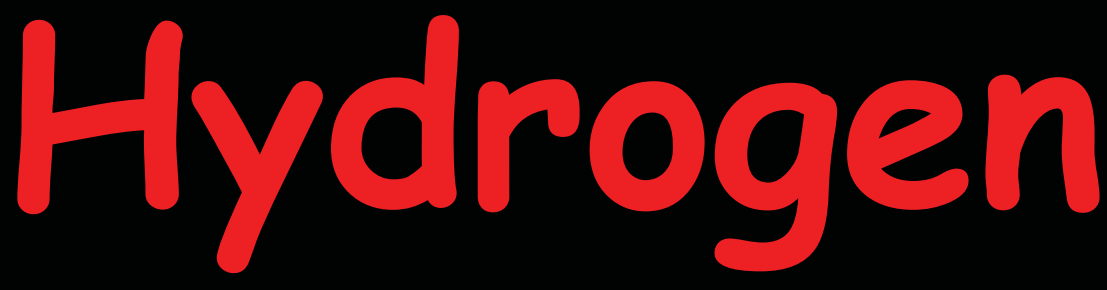

R. Pohl et al. Nature 466, 213, 2010

$\mathrm{JJ}$ and S. Roy 1008.3536 
- In ordinary $Q M 2 s_{1 / 2}$ and $2 p_{1 / 2}$ level degenerate

- This is a very peculiar feature of Coulomb's law,

$$
F(r) \sim \frac{1}{r^{2}}
$$

Deviations from Coulomb's law split $2 s_{1 / 2}$ and $2 p_{1 / 2}$

$\Rightarrow$ Measuring the Lamb shift is
a precise test of Coulomb's law! 
- Finite size of proton:

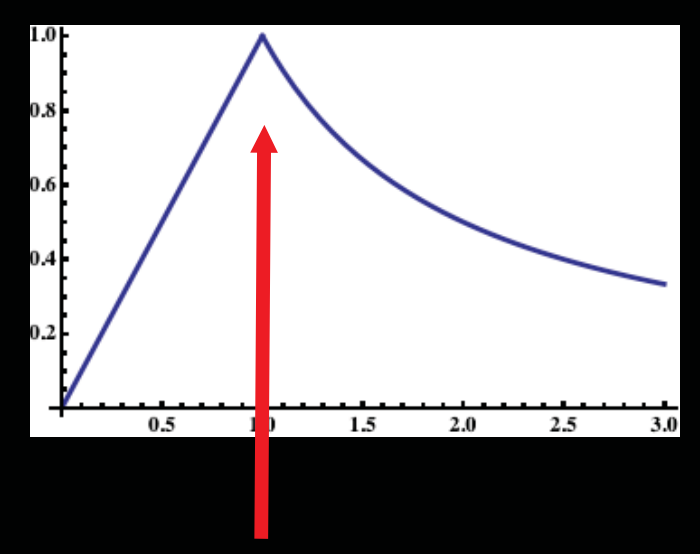

Rproton

- QED effects:

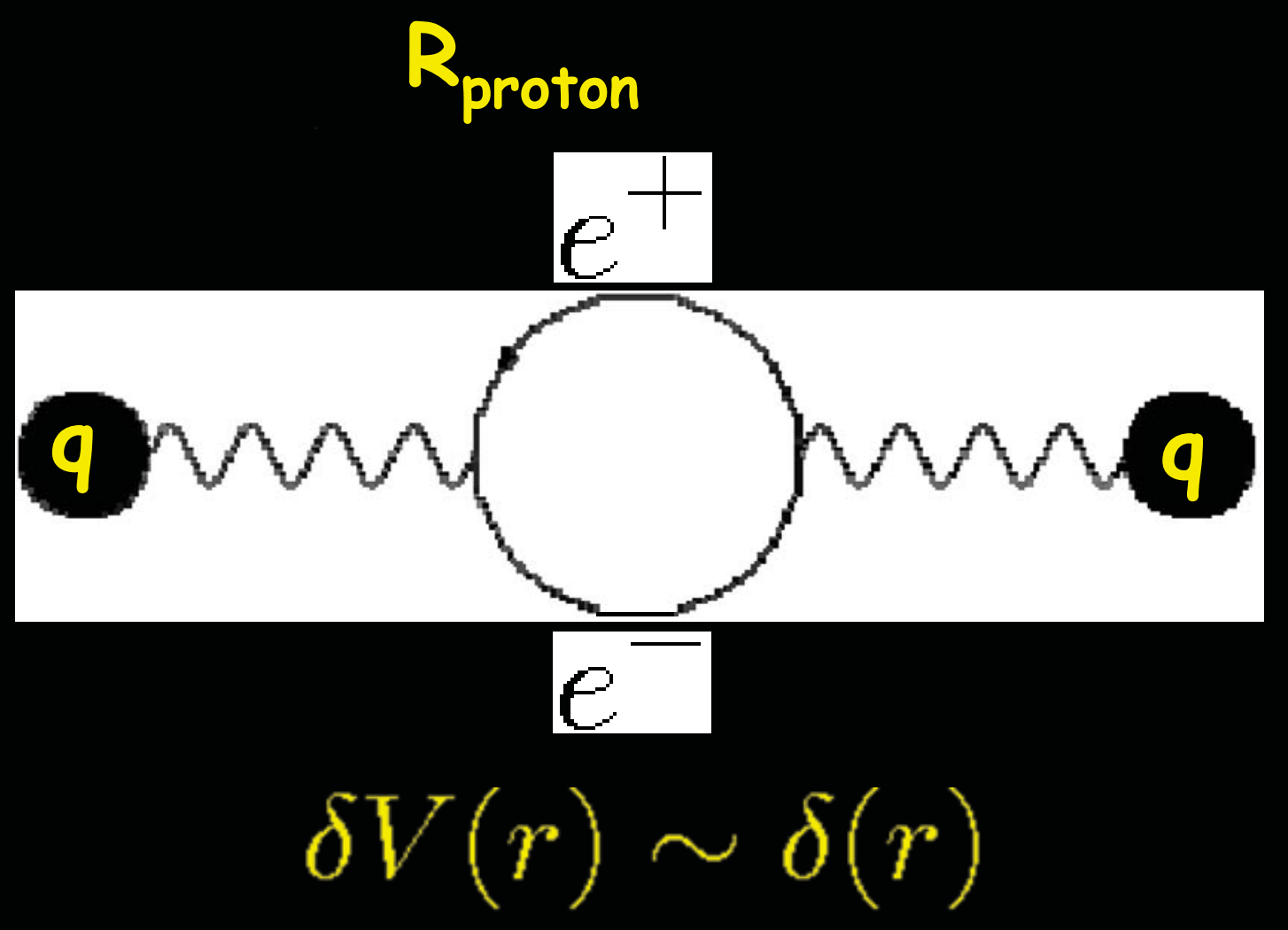




\section{Test for hidden photons and MCPs}

- WISPs modify Coulomb's law

- Hidden photons:

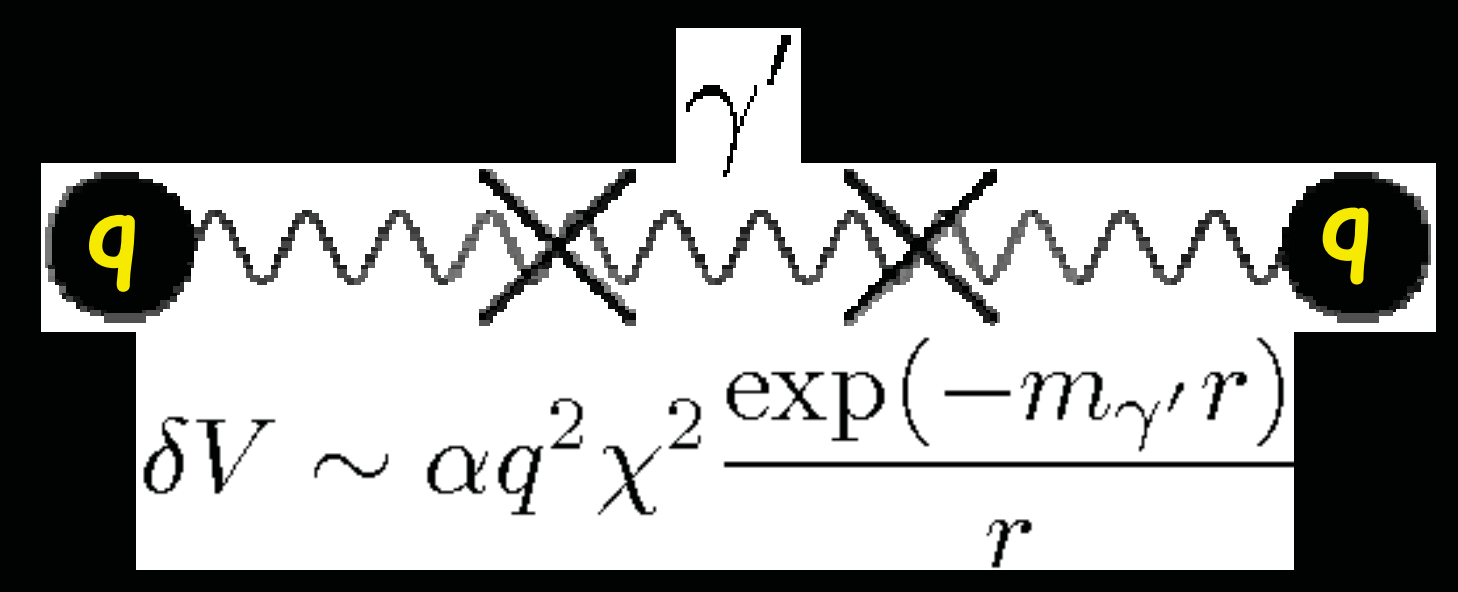

- Minicharged particles

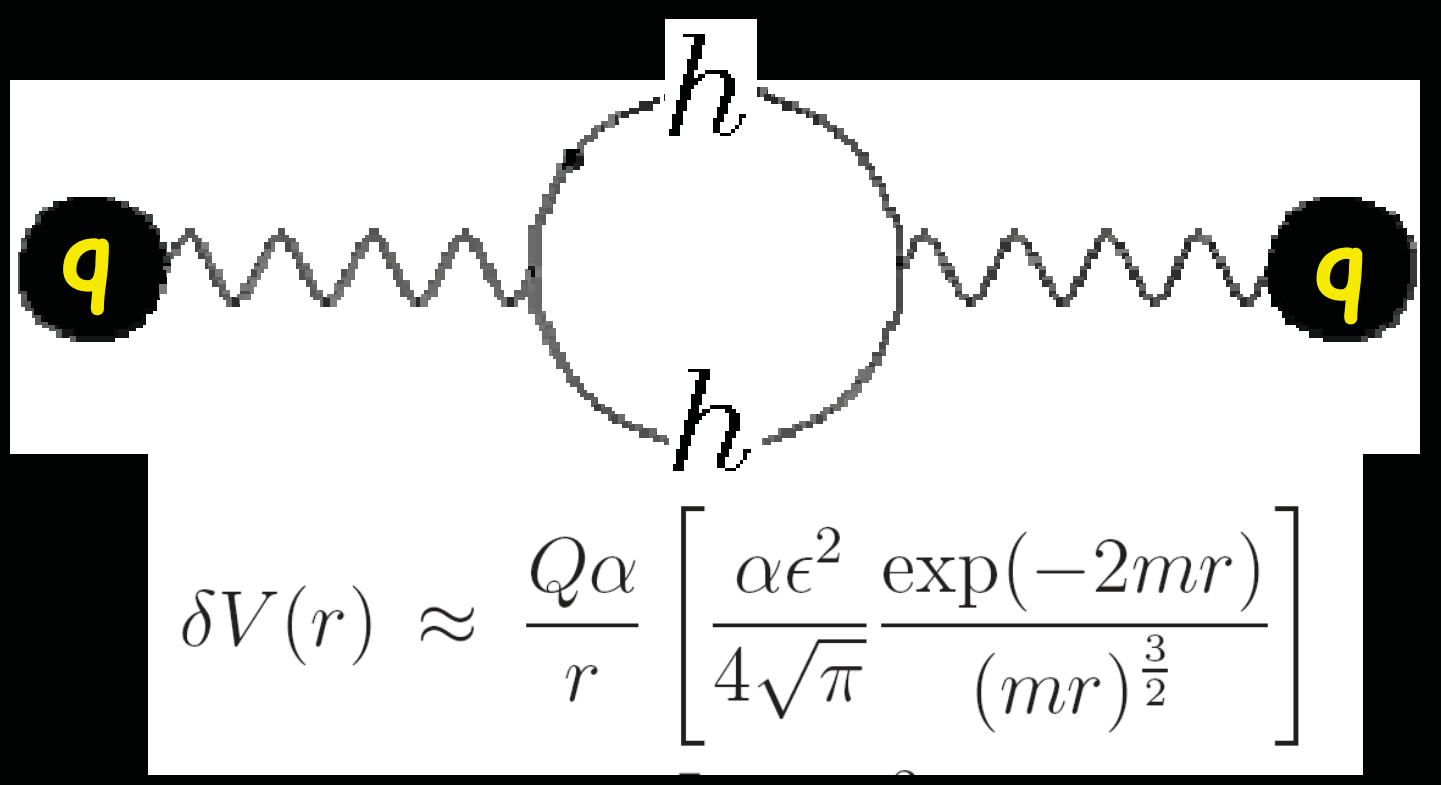




\section{New bounds...}

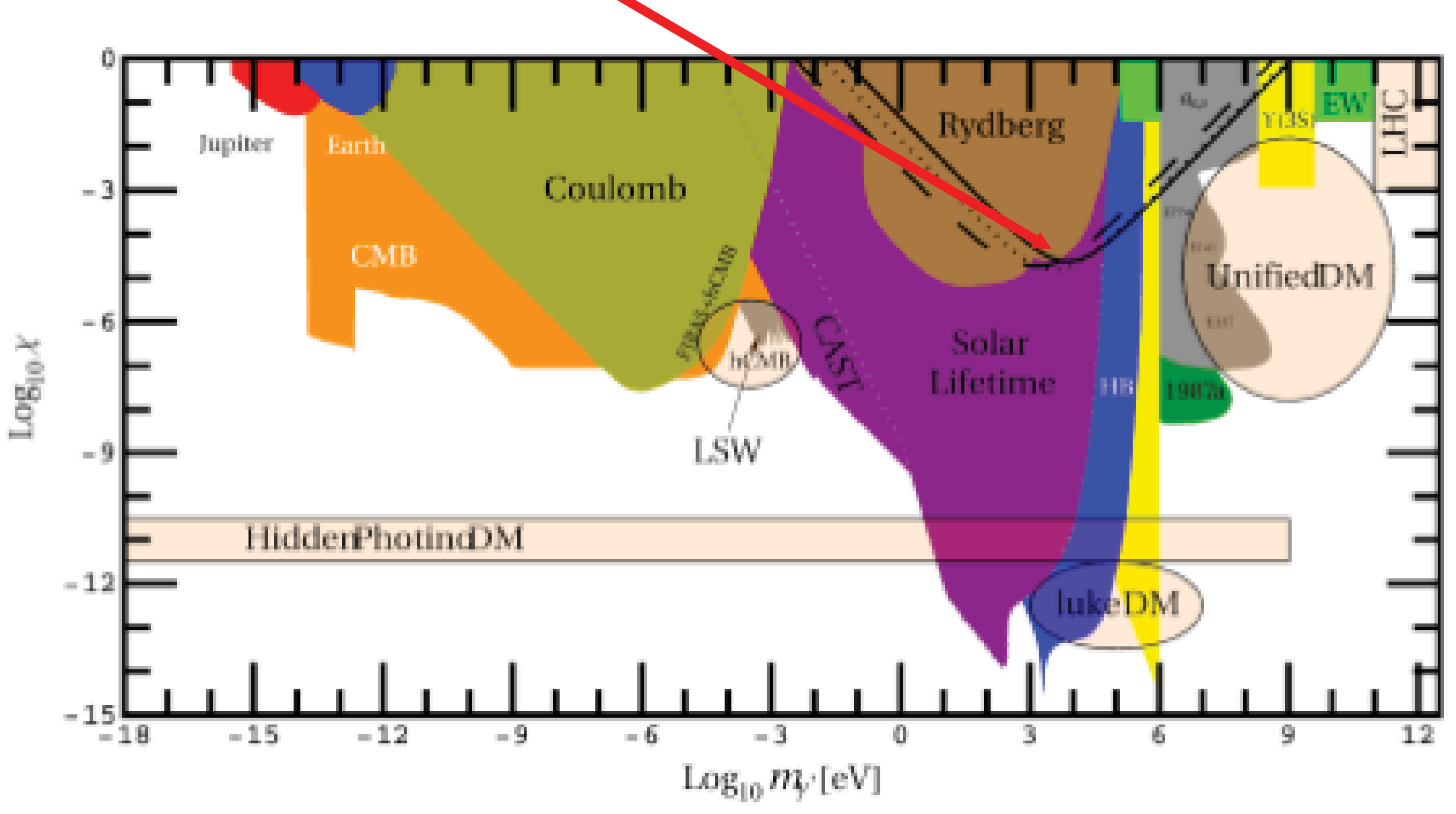


A puzzling measurement

- Use muonic hydrogen, i.e. $e^{-} \rightarrow \mu^{-}$

- Recently measured

$\Delta E_{s_{1 / 2}^{F=1}-p_{3 / 2}^{F=2}}=-206.295000(3) \mathrm{meV}$

- Compared to expected

$\Delta E_{s_{1 / 2}^{F=1}-p_{3 / 2}^{F=2}}=-205.984(062) \mathrm{meV}$

5 o deviation!!!! 
Can it be hidden photons???

$m(1 \mathrm{P})^{2} n$

- No!

\section{$e^{-} \rightarrow \mu^{-}$}

- Wrong sign: hydrogen would be more strongly affected!

Need more creative solution! 
Can it be hidden photons???

$m(1 \mathrm{P})^{2} n$

- No!

$$
e^{-} \rightarrow \mu^{-}
$$

- Wrong sign; hydrogen would be more strongly affected!

CNeed more creative solution!

$\longrightarrow$ Homework :-) 


\title{
Experimental Homework.
}

- WISPs may be visible in deviations of Coulomb's law

\author{
I \\ Something to Revive? \\ Tests of Coulomb's law
}




\section{Quite sensitive}

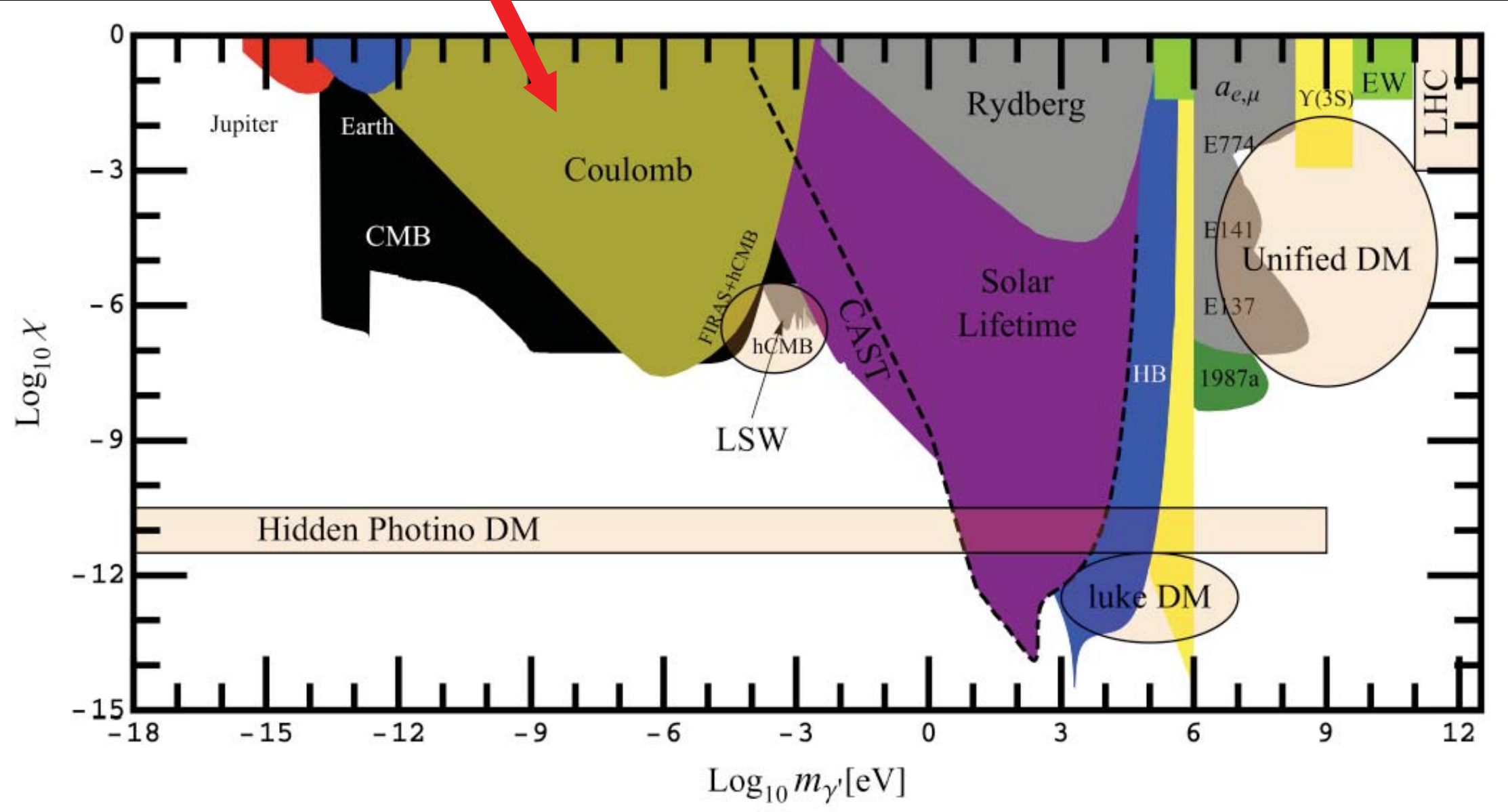

- Best experiment 40 years old!!!!!

E. Williams, J. Faller and H. Hill PRL 26, 721, 1971 


\section{Experimental Homework}

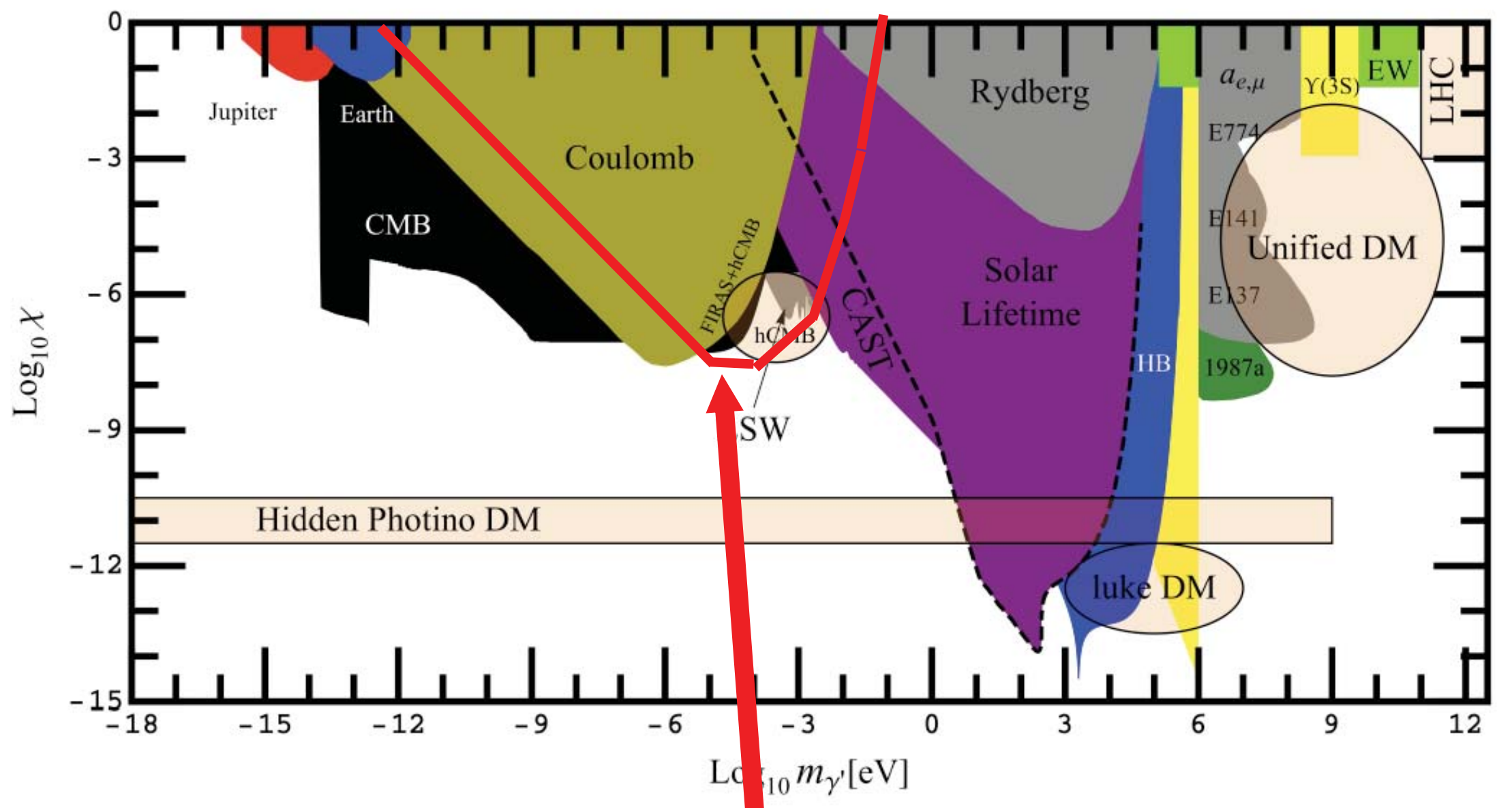

$\mathrm{cm}$-scale Cavendish test with precision few 10-15 


\section{Conclusions}




\section{Conclusions}

- Good Physics Case for Axions, WIMPs and WISPs

$\longrightarrow$ explore 'The Low Energy Frontier'

- Low energy experiments/observations test energy scales much higher than accelerators

$\rightarrow$ Complementary!

- May provide information on hidden sectors and thereby into the underlying fundamental theory

The Standard Model

Beyond the SM

(accessible to colliders)

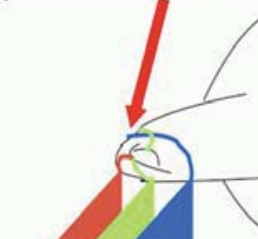

Hepe be Draqors
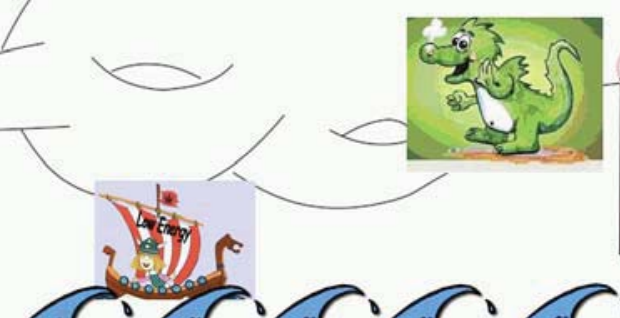

$9: 0: 3: 0$

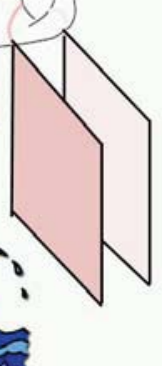

- Surprises (like Lorentz symmetry violation) possible! 